\title{
DEVELOPMENT OF A NON-FUSION SCOLIOSIS CORRECTION DEVICE
}

NUMERICAL MODELLING OF SCOLIOSIS CORRECTION 
This project, "A non-fusion scoliosis correction device", was supported by a grant from the Dutch Technology Foundation (STW), applied science division of NWO and the Technology Program of the ministry of economic affairs (project number 07618).

The printing of this thesis was financially supported by:

Stichting Technologische Wetenschappen (STW)

\section{Samenstelling promotiecommissie:}

Voorzittert en secretaris:

Prof. dr. F. Eising

Universiteit Twente

Promotoren:

Prof. dr. ir. G.J. Verkerke

Universiteit Twente

Prof. dr. A.G. Veldhuizen

Universitair Medisch Centrum Groningen

Assistent promotor:

Dr. ir. J.J. Homminga

Universiteit Twente

Leden:

Prof. dr. ir. N.J.J. Verdonschot

Universiteit Twente

Prof. dr. ir. A. De Boer

Universiteit Twente

Prof. dr. K. Ito

Technische Universiteit Eindhoven

Prof. dr. J.H. van Dieën

Vrije Universiteit Amsterdam

Prof. dr. ir. N.M. Maurits

Universitair Medisch Centrum Groningen

\section{Paranimfen:}

Tjitske Boonstra

Evelien Platvoet

Printed by: Ipskamp Drukkers BV, Enschede

ISBN: 978-90-365-3229-7

\section{Copyright () 2011 by G.J.M. Meijer, Enschede, The Netherlands.}

All rights reserved. No part of this publication may be reproduced or transmitted in any form or by any means, electronic or mechanical, including photocopy, recording or any information storage or retrieval system, without permission in writing from the author. 


\title{
DEVELOPMENT OF A NON-FUSION SCOLIOSIS CORRECTION DEVICE
}

\section{NUMERICAL MODELLING OF SCOLIOSIS CORRECTION}

\author{
PROEFSCHRIFT
}

ter verkrijging van

de graad van doctor aan de Universiteit Twente,

op gezag van de rector magnificus,

prof. dr. H. Brinksma,

volgens besluit van het College voor Promoties

in het openbaar te verdedigen

op vrijdag 14 oktober 2011 om 12.45 uur

door

Gerarda Johanna Maria Meijer

geboren op 6 december 1978

te Oldenzaal 
Dit proefschrift is goedgekeurd door:

Prof. dr. ir. G.J. Verkerke (promotor)

Prof. dr. A.G. Veldhuizen (promotor)

Dr. ir. J.J. Homminga (assistent promotor)

ISBN: 978-90-365-3229-7

Copyright $\odot 2011$ by G.J.M. Meijer, Enschede, The Netherlands 



\section{Table of Contents}

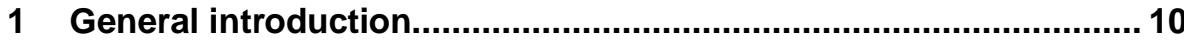

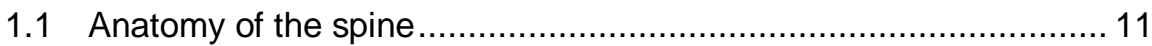

1.2 Adolescent Idiopathic Scoliosis.............................................. 13

1.3 Mechanical behaviour of the spine ......................................... 16

1.4 The use of numerical models in optimizing scoliosis correction ...... 17

1.5 Aim and outline of this thesis ................................................ 19

2 Models of the spine and trunk and their validation processes....... 24

2.1 Structure of the model ......................................................... 25

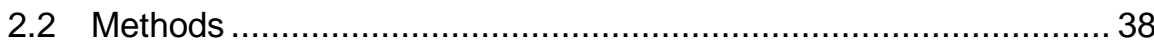

2.3 Validity of the models ....................................................... 40

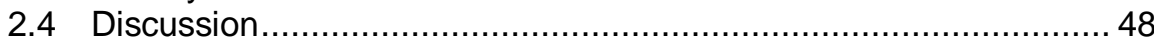

2.5 Conclusion on usability and validity of the model.......................... 51

3 The effect of three-dimensional geometrical changes during adolescent growth on the biomechanics of a spinal motion segment. 58

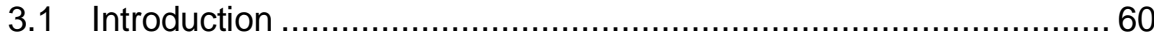

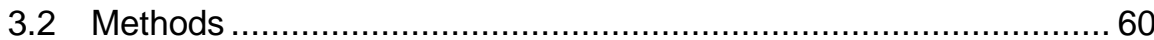

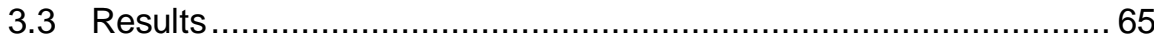

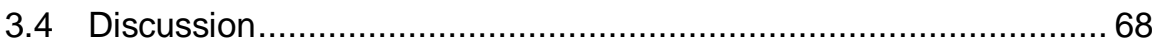

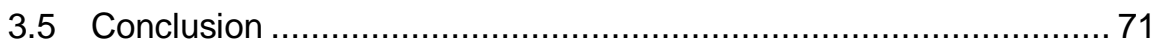

4 Influence of interpersonal geometrical variation on spinal motion segment stiffness - implications for patient-specific modelling............76

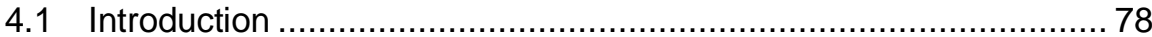

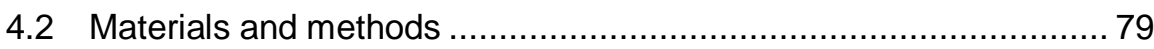

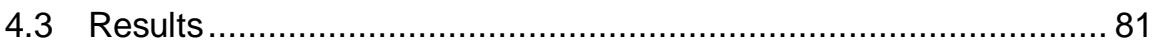

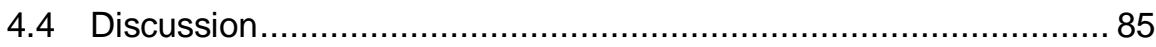

5 Influence of costovertebral joints on the stiffness of the spine ..... 92

5.1 Introduction ......................................................................... 94

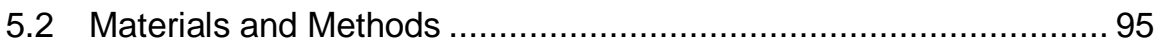

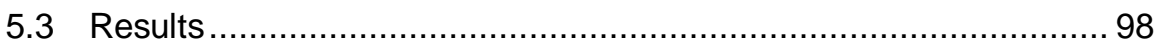

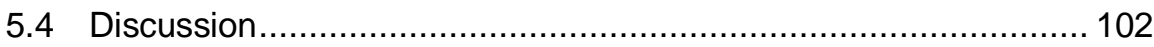

6 Mechanical role of the spine, ribcage and intra-abdominal pressure

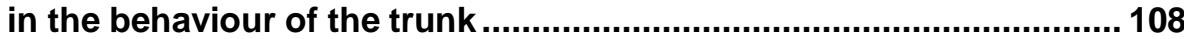

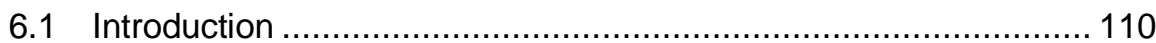

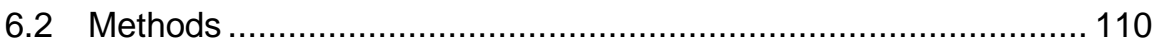

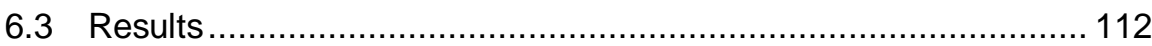

6.4 Discussion and conclusion................................................. 116 
7 Is scoliosis induction a good model for scoliosis correction? .... 120

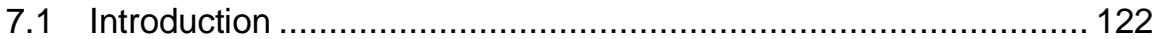

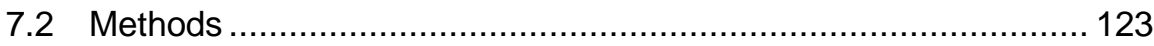

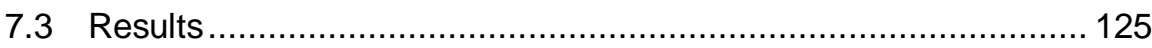

7.4 Discussion and conclusion................................................. 127

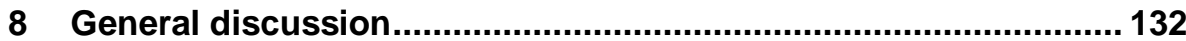

8.1 Discussion of the model....................................................... 133

8.2 Discussion of the results ...................................................... 136

8.3 Concluding remarks............................................................ 140

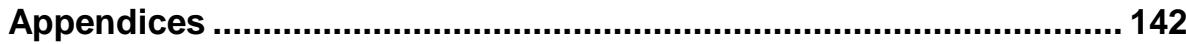

A Quantitative data per vertebral level....................................... 143

B Definition of planes and local coordinate system ....................... 146

C Shape of the endplates....................................................... 148

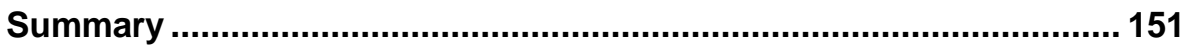

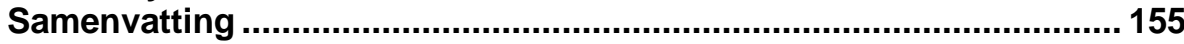

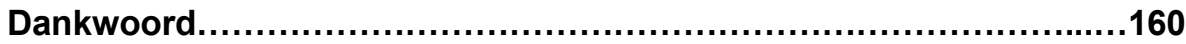

About the author........................................................164

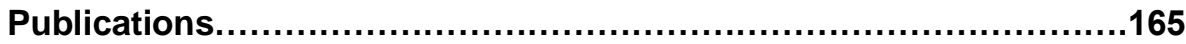



Chapter 1

General introduction 
Scoliosis is a three-dimensional deformity of the spine and occurs most in adolescent girls. Without treatment scoliosis progression can lead to a life-threatening situation, since the heart and lungs become oppressed. So correcting the spine is necessary. This PhD-thesis is part of a multi-disciplinary project aimed at the development of an implantable, non-fusion scoliosis correction device. The project consists of three PhD projects: 1) the design and prototyping of the new scoliosis-correction implant (Martijn Wessels), 2) in vitro tests on human and porcine spines to determine biomechanical spine properties and animal experiments to test the prototype (Iris Busscher) 3) development of a numerical model of the mechanical behaviour of the spine and trunk to optimize the design of the implant (this thesis).

In this first chapter the What, Why and How of the research in this thesis are outlined. Background information about the anatomy of the healthy spine (1.1), general information about scoliosis (1.2) and the biomechanics of the spine (1.3) is given. In section 1.4 the use of biomechanical models in optimizing scoliosis correction is discussed and the need for a new model is motivated. In the last section the aim and outline of the thesis is explained.

\subsection{Anatomy of the spine}

The human spine is a complex structure with typically shaped bony segments (the vertebrae), separated by flexible segments (intervertebral discs). Although the vertebrae gradually change shape along the spine, a division into five regions is

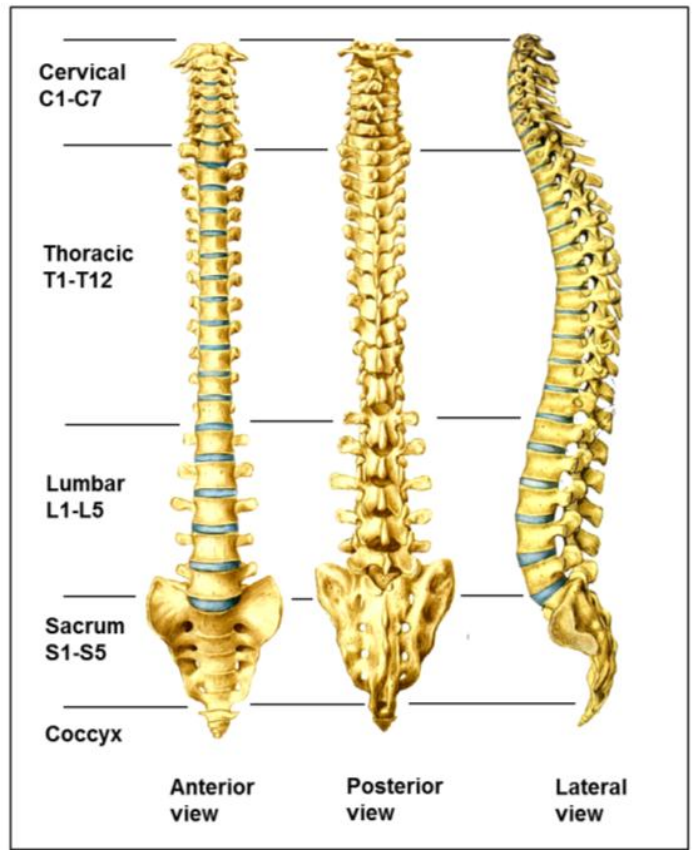

Figure 1: Anatomy of the spine. ${ }^{1}$ 
typically made, with a numbering of the vertebrae per region: cervical (C1-C7), thoracic (T1-T12), lumbar (L1-L5), sacral (S1-S5) and coccyx (figure 1). In the sagittal plane, the spine is curved; convex anteriorly (lordosis) in the cervical and lumbar region and convex posteriorly (kyphosis) in the thoracic and sacral region (figure 1, right). In the coronal plane the normal spine appears straight and symmetrical (figure 1, middle and left).

All vertebrae consist of the same elements, although elements can be more or less pronounced in certain regions, mainly because of differences in mobility and variation in attachments of muscles and/or ribs.

A typical vertebra (figure 2) consists of a body (corpus) in the front and a hollow ring with several processes (vertebral arch) in the back. This vertebral arch encloses the vertebral foramen, through which the spinal cord ascends. The top and bottom of a vertebra consist of a layer of cancellous bone, and are called endplates. The endplates also form the interface with the intervertebral disc. The core of this disc (nucleus pulposes) is gel-like, providing damping, while the surrounding ring (annulus fibrosus) contains oblique directed fibres to limit deformation. The posterior parts of two adjacent vertebrae articulate via two facet joints (left and right); the superior articular process of the lower vertebra articulates with the inferior articular process of the upper vertebra.

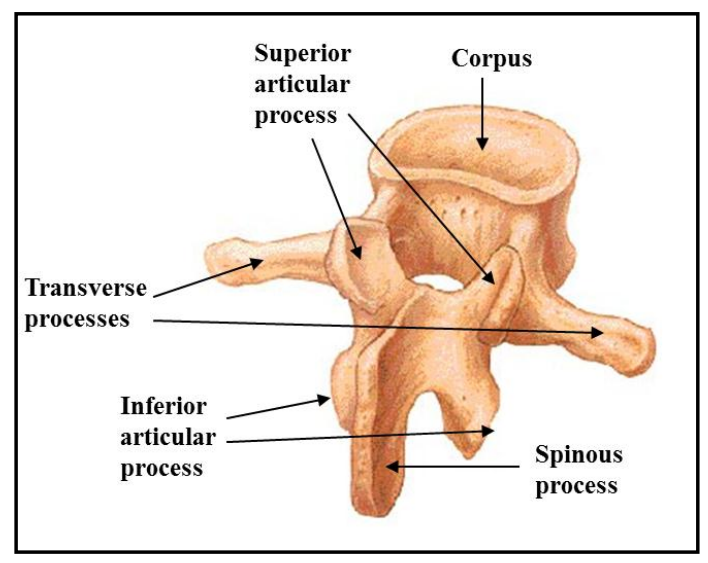

Figure 2: Typical vertebra. ${ }^{2}$

The smallest unit representing the mechanical behaviour in a given region of the spine is called a motion segment and consists of two adjacent vertebrae including the facet joints, the intervertebral disc and seven spinal ligaments (figure 3). 


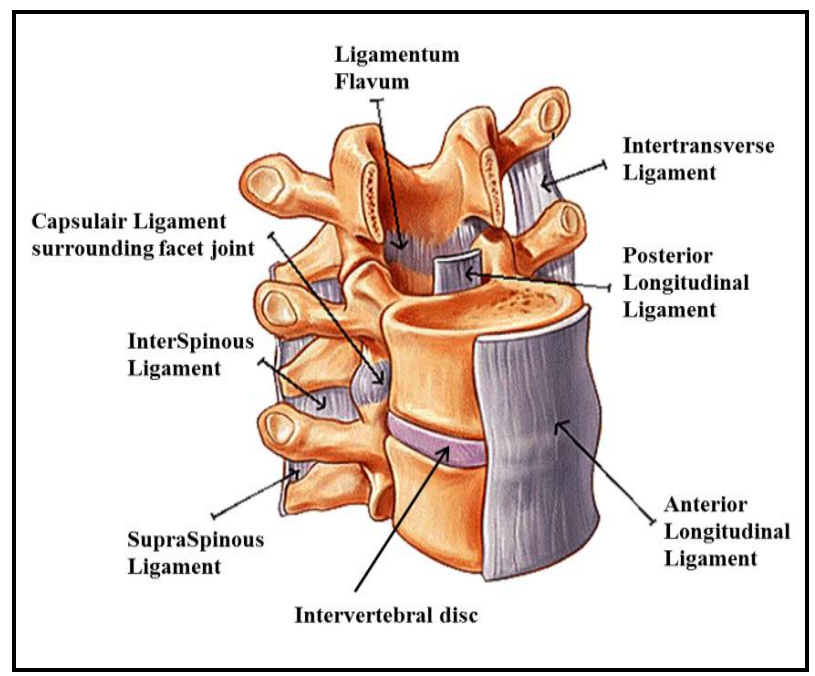

Figure 3: Motion segment: two adjacent vertebrae including the facet joints, the intervertebral disc and seven spinal ligaments.

\section{$1.2 \quad$ Adolescent Idiopathic Scoliosis}

Scoliosis is a deformity of the trunk, mainly characterized by a lateral deviation of the spinal column in combination with axial rotation of the vertebrae. This axial rotation of the vertebral bodies is towards the convexity of the curve (figure 4). Although adult and infantile forms of scoliosis exist, the most common form of scoliosis is Adolescent Idiopathic Scoliosis, which is also the focus of this thesis.

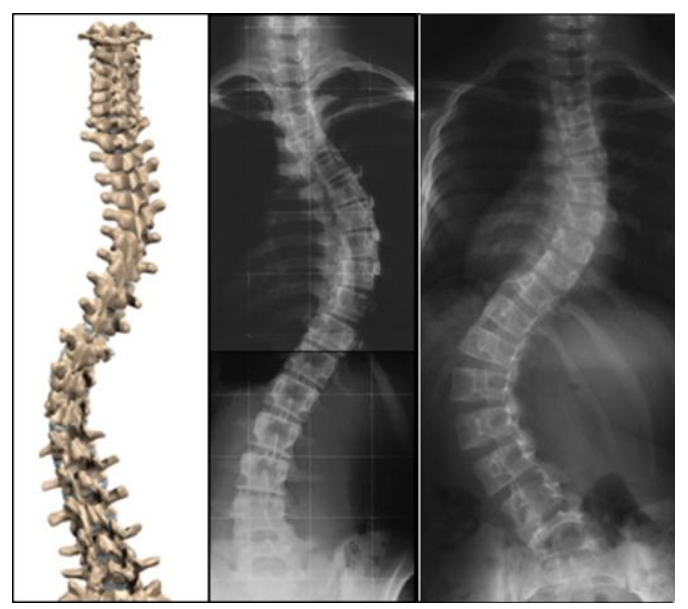

Figure 4: The scoliotic spine. Left: schematic representation of a thoracic scoliosis, note the rotation of the vertebrae ${ }^{4}$ middle: $x$-ray of thoracic scoliosis ${ }^{5}$; right: $x$-ray of lumbar scoliosis ${ }^{5}$. 
Idiopathic refers to the unknown aetiology of the deformity, which is the case for approximately $80 \%$ of the scoliosis cases. Less common types of scoliosis have a congenital, neuromuscular or traumatic origin. Although multiple areas of research, including connective tissue, neuromotor mechanisms, hormonal system, genetics and biomechanics, have been explored for a potential relationship to the cause of idiopathic scoliosis, no clear evidence has been found for a single factor as the main cause of this disorder. The main difficulty of most studies is to determine whether the observed abnormalities are primary or secondary features in the scoliotic deformity. The current consensus on the aetiology is that it is multifactorial, with a genetic component that still needs specifying, and a key role for biomechanics and growth during progression ${ }^{6-9}$.

Adolescent refers to the onset of the deformity: between age ten and skeletal maturity. Besides adolescent scoliosis, infantile scoliosis (detection before age of four) and juvenile scoliosis (detection between four and ten years) are distinguished. A simplified division into early-onset scoliosis (before age ten) and late-onset scoliosis (after age ten) is also sometimes made.

In early stages of scoliosis the first noticeable change is lateral wedging of the spine, accompanied by axial rotation of the vertebral bodies and discs and changing vertebral-rib-angles ${ }^{10,11}$. When scoliosis progresses, the vertebrae themselves will also deform and the deformed spine, in turn, deforms the posterior part of the rib cage $^{12}$; the ribs are slowly pushed aside and a rib hump (gibbous) is formed on the convex side of the scoliotic curve.

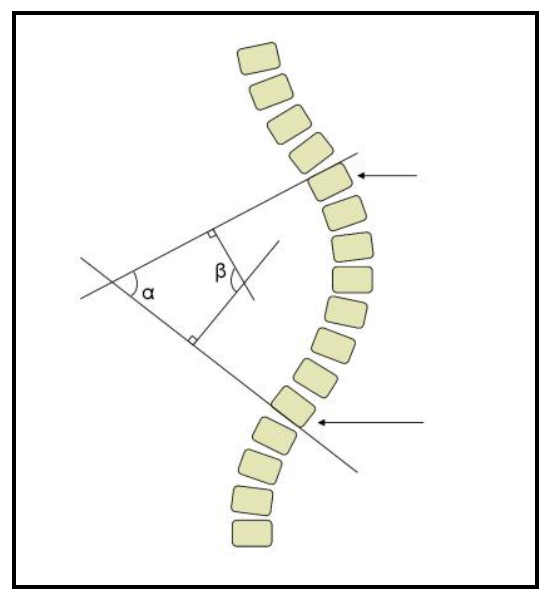

Figure 5: Determination of Cobb angle in the frontal plane of the spine.

The severity of scoliosis is often quantified by the Cobb angle, as described in figure 5 . This angle is defined as the angle between the two most rotated vertebrae in the frontal plane (indicated with arrows in figure 5) and can be determined directly $(\alpha)$ or indirectly, using perpendicular lines $(\beta)$. Clinically, scoliosis is diagnosed when the lateral curvature of the spine is larger than 10 degrees as measured using the Cobb method, which has been defined by the Scoliosis Research Society ${ }^{13}$. Up to 25 
degrees the deformity is considered as mild scoliosis, between 25 and 45 degrees as moderate and from 45 degrees as severe scoliosis. Although the distortion of the spine and trunk is three-dimensionally, the deformity is thus measured only twodimensionally in an anterior-posterior radiograph of the spine.

Scoliosis progresses mainly during the adolescent growth spurt, but large curves can also progress in adult life. The three main risk factors for progression are patient gender, remaining spinal growth and the severity of the scoliosis at the time of diagnosis.

Mild scoliosis can be found in 2 to 3 per cent of the children between 10 and 16 year, and the prevalence for Cobb angles larger than 30 degrees is $0.2-0.3 \%$. The female to male ratio also varies with the magnitude of the curve: the ratio is equal among patients with a mild scoliosis, but in curves of more than 30 degrees, $90 \%$ of the patients are female $e^{14-17}$.

Scoliotic curve patterns are classified as single (C-shape) or double curve (S-shape), depending on the shape in frontal plane. A second classification can be made based on the location of the apex, which is the most laterally deviated disc or vertebra. Thoracolumbar and thoracic curves are most common ${ }^{14,15,17-19}$.

The clinical treatment of scoliosis depends on the severity of the curve (Cobb angle), the remaining growth (age) and the progression of the curve (increase of the Cobb angle $)^{20-22}$.

In mild cases with little curve-progression the patient is simply monitored, or treatment consists of physiotherapy and exercises. Although the benefits of physical therapy and exercise seem intuitive, it has not been shown that this treatment alters the natural history of scoliosis.

In mild and moderate scoliosis with progression of the curve, bracing is considered a proper treatment to limit progression of the scoliosis. Major disadvantage of this treatment is that curve progression will reoccur when the brace is no longer used and full correction is not achieved ${ }^{23,24}$.

When Cobb-angles exceed 40 degrees and the curve is progressive, implanted metal systems are used to correct the deformity by fusion of the vertebrae. Major disadvantage of this form of treatment is that it can be only started when growth is complete or almost complete. Moreover, mobility of the spine is greatly reduced.

In the current project a new correction implant, in which the vertebrae are not fused, is developed for progressive scoliotic curves. The main advantage is that the implant can be used to correct scoliosis while the growth is still ongoing. Due to the earlier application of the correction, the deformity that has to be corrected will be less and smaller forces can be used compared to those in current surgical treatments. Of course, the preservation of the mobility of the spine is also an improvement of the quality of life for the patients that are treated in this way.

The most fundamental questions for the design of the implant are related to the mechanics of the spine and ribcage. The biomechanics of the spine not only plays a large role in the progression of scoliosis ${ }^{6-9}$, but also in the correction that is realised by surgery ${ }^{25-29}$, and in the risk of fusion of the vertebrae ${ }^{30,31}$. The mechanical behaviour of the spine is therefore discussed in more detail in the next section. 


\subsection{Mechanical behaviour of the spine}

Because the motion allowed between any two vertebrae is small, spinal movement always involves movement of multiple motion segments. The various planes of the human body and the movement- and loading-directions are explained in Appendix A. In theory, a motion segment has six degrees of freedom; 3 rotational and 3 translational. Since the rotational degrees of freedom cause larger displacement than the translational ones and the rotational movements are more relevant in scoliosis, the focus in this thesis will be on the rotational movements. The three rotational movements are flexion/extension (forward/backwards bending), lateral bending (sideways bending) and axial rotation (around the length-axis of the spine). The range of motion allowed at each motion segment is governed by anatomical constraints that vary between the regions of the spine and differ for the directions of movement. Mainly the shape and orientation of the facet joints determine (restrict) the mobility of the spine (figure 6).

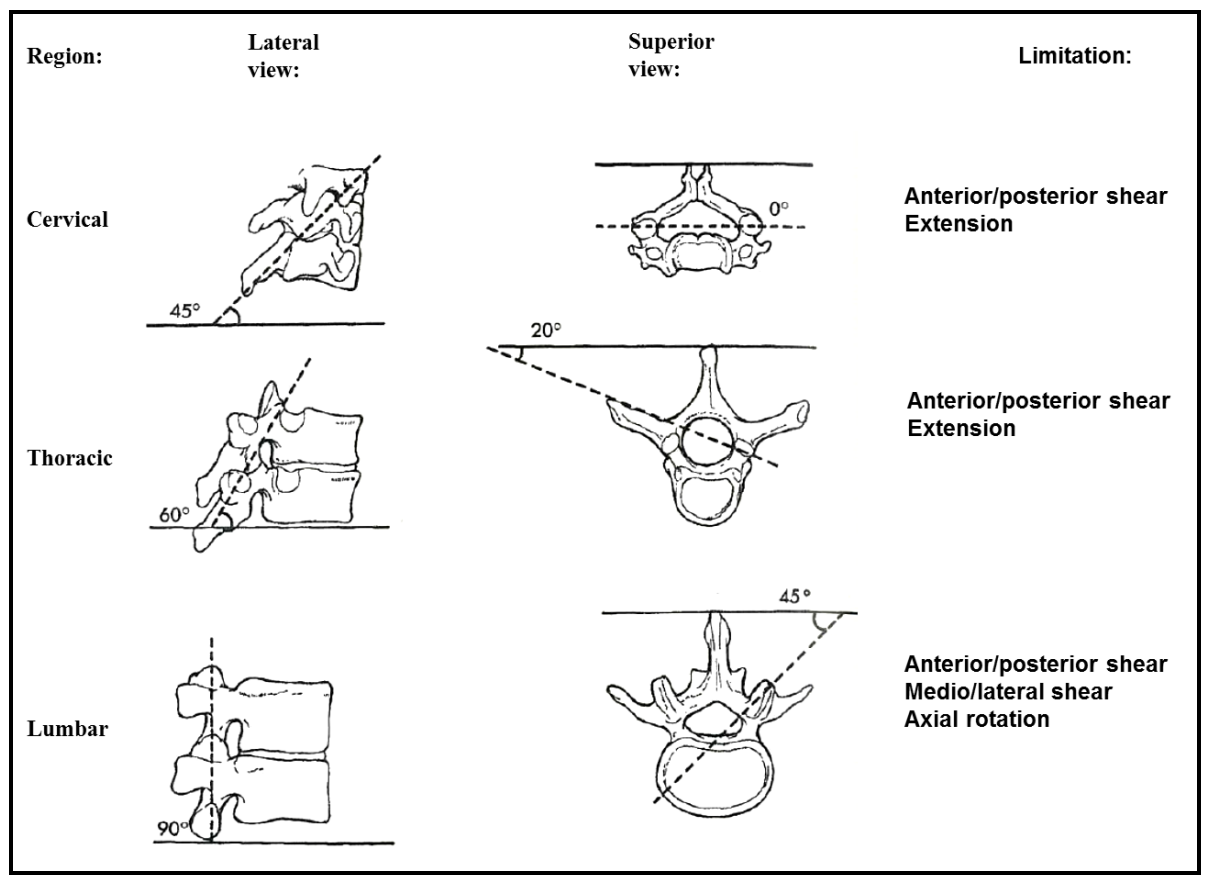

Figure 6: Orientation of the facet joints and the following limitation on movements varies for the different regions of the spine. ${ }^{32}$

In the thoracic region, the motion of the spine is also limited by the ribs, which are posteriorly attached to the vertebrae and anteriorly to the sternum. And of course muscles and ligaments restrain the motion and provide stability. 
It should be kept in mind that motions in real life are often a combination of motions. And a motion in one direction influences the mechanical behaviour of the spine in other directions. A clear example is the presence of gravity during daily life, resulting in compression of the spine, which again increases the stiffness of a motion segment for rotational movements ${ }^{33}$.

Many in vitro experiments have been carried out to analyse the biomechanical behaviour of the spine, on various levels. Motion segments ${ }^{34-39}$, multi-segment level $40-42$, thoracic spines including the ribcage ${ }^{43}$ and even complete thoracolumbar spines ${ }^{44}$ have been tested, providing data on the mechanics of the various regions and structures of the spine. However, the range in the reported data is large. This is due to both the large variation in testing methods and the large inter-personal differences in spinal stiffness. Another large disadvantage for the application of the experimental data in scoliosis research is the very limited availability of adolescent cadaveric material. As a result in vitro studies have to rely on aged cadaveric material, with the accompanying degeneration. An extrapolation to the biomechanical aspects for adolescents is not straight-forward, as both geometrical and material changes have to be considered.

The use of in vivo measurements may seem a solution for acquiring adolescent data, but the actual loading during these tests due to muscles and support forces are unknown. Only range of motion can be determined under maximum voluntary bending; a determination of the stiffness of the spine from these data is impossible. Also, again, the range in reported range of motion is large. This can be explained by variations in testing methods, muscle activation patterns and inter-personal differences in stiffness of the spine.

\subsection{The use of numerical models in optimizing scoliosis correction}

Numerical models have been used in spinal research since the 1970's and have developed ever since ${ }^{45}$. One of the advantages of numerical models is that one-andthe-same "specimen" can be tested under various (loading) conditions without loss of biomechanical properties due to testing time or damage. Another large advantage is that the naturally occurring range in patient-specific properties can be either taken out of the analysis or analysed independently. Also, numerical models can provide information that is difficult or impossible to obtain from experiments, such as stress distribution throughout the disc and vertebral bodies and models can also be used for experiments that cannot be carried out for practical or ethical reasons.

Because of these advantages, numerical models can assist in the design and development of spinal instrumentations. Another advantage is that these designs can be tested without actually having to first make a prototype. Sensitivity studies can be used to analyse the effect of variations of the design of the implant before actually manufacturing it, and numerical models can thus be used to optimize the design.

At the start of this project, state-of-the-art models either consisted of a few segments modelled with great detail or represented the complete spine with limited detail ${ }^{45}$. Since scoliosis affects large parts of the thoracolumbar spine ${ }^{14,15,17,19}$ and the ribcage ${ }^{10,12,46}$, a model that represents the total thoracolumbar spine and ribcage is needed. In complete spine models, however, the motion segments were oversimplified, often represented by a single stiffness matrix, representing the total 
intervertebral disc, spinal ligaments and sometimes even parts of ribcage. Especially for scoliosis research, this representation was not sufficient. When the deformed disc is represented by a single stiffness matrix, the properties of the stiffness matrix need to be corrected for the changed properties due to the deformity, and these changes are not straight-forward nor small. A patient-specific correction of this stiffness matrix has been proposed, based on radiographs of maximum voluntary bending tests ${ }^{47}$. Unfortunately, as the loading conditions during these tests are unknown, the tests are unsuitable as a true flexibility measurement. Furthermore, only one movement direction is analysed (lateral bending) and this makes the scaling-method inaccurate for the other movement directions, since the various anatomical structures are not modelled in a physiological way, but as a combined matrix.

Since all models at that time had shortcomings for our goal, a new model was created. This model needed to represent the essential biomechanics of the spine and trunk on a macroscopic level, including non-linearity in both geometry and material properties.

A prerequisite of numerical models is validation before they can be used with confidence. For this, comparison with controlled in vitro experiments is needed, but sensitivity analyses to consider the effects of the uncertainty in the input parameters are also important ${ }^{48}$. Verification of the method is also necessary; not only the accuracy of the chosen mesh size has to be checked, but also convergence of the equilibrium equations and correct influence of numerical damping has to be verified. The actual checks depend on the chosen numerical method.

For finite element codes, implicit and explicit time integration can be distinguished. In the implicit method, the total coupled set of the equations of motion is solved. For each time step, an iteration procedure is employed until a user-specified convergence criterion is met. This results in an accurate (the acceptable error is defined by the user) and unconditionally stable, but time-consuming, solution.

In the explicit method, an uncoupled set of equilibrium equations is solved, by making the coefficient matrix diagonally. Although this method is faster, it is conditionally stable, having a critical time step which must not be exceeded. As the critical time step is quite small, a great number of time steps are required to simulate the whole process. A drawback of this method is that an iteration procedure to converge to equilibrium is not possible, resulting in errors in the equilibrium conditions.

Because of the large number of elements required in the model, solving a coupled set of equations is impractical, and an explicit time integration scheme is adopted.

The finite element model was made in Pam-Crash software (Version 4.5, ESI-Group, Paris, France) since it met all requirements (combining finite element mesh with rigid bodies, elaborate contact definitions, non-linear material properties, fibre reinforced materials and muscle activation options) and a multi-body model of a $5^{\text {th }}$ percentile female was included (described in section 2.1.2) that could be used as a basic geometry of the trunk. 


\subsection{Aim and outline of this thesis}

Main goal of this thesis is to present a numerical model of an average adolescent spine that quantifies the various biomechanical aspects that are important in scoliosis correction. This model will help to optimize the development of a new scoliosis correction implant. In addition, it will also increase our basic knowledge on the biomechanics of the spine and trunk.

The final model is presented in chapter 2 . First the structure of the model and the comparable anatomical structures are explained, followed by the validation for various aspects of the model. Finally, the assumptions and simplifications are discussed, resulting in a conclusion on the usability of the model.

Since adolescent idiopathic scoliosis is the aim of our research, the spine model has to be representative for an adolescent spine. However, for validation only adult spines are available. In the third chapter, it is therefore analysed what the effects of growth on the biomechanics of a motion segment are.

In the fourth chapter, the effects of patient-specific geometry on the biomechanics of the spine are analysed. Since our motion segment model is a generic model, it is easy to vary the various geometrical aspects and analyse the effects on the biomechanics of the spine. This study will help in determining which geometrical aspects of the model need patient-specific adaptation and at which accuracy level, to give an accurate determination of the patient-specific stiffness.

In chapter 3 and chapter 4 , a model of a motion segment is used, but for the optimizing of the design of the implant, the model needs to represent a larger part of the spine. Since scoliosis mainly occurs in the thoracic and thoracolumbar region $^{14,15,17,19}$, this is the region of interest. In progressive scoliosis, ribcage deformation also occurs. For a representative model for progressive cases of scoliosis, the ribcage is thus also essential.

In chapter 5 a first step towards a larger model is made. A model containing three thoracic motion segments and the posterior part of the connected ribs is used in this study. For the validation of the model, the in vitro experiments carried out within this project ${ }^{49}$ are used. Since in the thoracic region of the spine, the costovertebral ligaments are anatomically intertwined with the spinal ligaments and the intervertebral disc, their removal is time-consuming and potentially harmful to the quality and biomechanical behaviour of the specimen ${ }^{50}$. For this reason, most in vitro tests leave the posterior part of the ribs (about $3 \mathrm{~cm}$ ), intercostal muscles, costovertebral joints and ligaments attached, assuming this to be a good representation of the isolated spine. With our models we tested this hypothesis. This chapter also provides basic knowledge about the relative influence of each of the costovertebral connections (costotransverse joints and ligaments, costovertebral joints and ligaments and intercostal muscles) on spinal mechanics.

As remarked before, both the ribcage ${ }^{43,51}$ and intra-abdominal pressure ${ }^{52-56}$ have a large effect on the stability and stiffness of the spine. However, the effect of neither of these have ever been analysed in adolescents. Therefore, in chapter 6 , the effects of the ribcage and intra-abdominal pressure (IAP) on the biomechanics of the ten year old spine are analysed. 
For the modelling of this IAP a new method is used. Previous biomechanical models used a simplified representation of the IAP, by representing it as a single force in the middle of the diaphragm ${ }^{52,53,56}$. Since it is not agreed upon whether the stabilising effect is due to the contraction of the abdominal muscles, and IAP merely is a byproduct of this, or IAP itself is the main stabiliser, this representation seems an oversimplification. Therefore, in the current model, the intra-abdominal pressure is represented by an incompressible volume in the shape of the intra-abdominal cavity, with an overpressure. In this way, the direct effect of the intra-abdominal pressure on the vertebrae is represented, as well as the upward force on the diaphragm and ribcage, and the contraction of both the abdominal and dorsal muscles.

In chapter 7 the performance of the designed implant is tested as the final goal of the numerical model. The short term and long term outcome for the lateral, sagittal and axial deformity are analysed. Furthermore, a comparison of scoliosis correction with scoliosis induction, which is used in animal experiments, is made. Since scoliosis does not occur in animals, in current animal experiments scoliosis is induced rather than corrected ${ }^{31,57,58}$, which will also be done in this project. From a mechanical point of view, the comparison between scoliosis correction and induction is not straightforward. Therefore, we want to compare the mechanical behaviour of a healthy trunk in which scoliosis is induced to a scoliotic trunk in which scoliosis is corrected, to analyse whether these in vivo scoliosis induction experiments are a good representation for scoliosis correction.

A general discussion is presented in the last chapter. The strength and limitations of the model are summarized and recommendations for improvement of the model are given. The results and their (clinical) implications are discussed, resulting in speculations on the future directions of modelling in scoliosis research and concluding remarks. 


\section{References}

[1] R. Putz, R. Pabst (eds) (1993) Sobotta atlas of human anatomy., vol 2: Trunk, Viscera, Lower Limb. 20th edn. Bohn Stafleu Van Loghum Houten, The Netherlands

[2] University of Maryland Spine Program. A patient's guide to anatomy and function of the spine. http://www.umm.edu/spinecenter/education/anatomy and function of the spine.htm. Accessed 15-12-2010

[3] A.A. White, M.M. Panjabi (1978) Clinical Biomechanics of the Spine. 1st edn. Lippincott Philadelphia, USA

[4] University of Maryland Spine Program. A patient's guide to adolescent idiopathic scoliosis. http://www.umm.edu/spinecenter/education/adolescent idiopathic scoliosis.htm. Accessed 16$12-2010$

[5] Eurospine Society. Types of scoliosis. http://www.eurospine.org/p31000269.html. Accessed 20-12 2010.

[6] A.G. Veldhuizen, D.J. Wever, P.J. Webb, 'The aetiology of idiopathic scoliosis: biomechanical and neuromuscular factors'. European Spine Journal Vol. 9, pp. 178-184, 2000.

[7] J.-W.M. Kouwenhoven, R.M. Castelein, 'The pathogenesis of adolescent idiopathic scoliosis: Review of the literature'. Spine Vol. 33, pp. 2898-2908, 2008.

[8] T.G. Lowe, M. Edgar, J.Y. Margulies, N.H. Miller, V.J. Raso, K.A. Reinker, C.-H. Rivard, 'Etiology of idiopathic scoliosis: Current trends in research'. J Bone Joint Surg Vol. 82-A, pp. 1157-1168, 2000.

[9] K. Cheung, T. Wang, G. Qiu, K. Luk, 'Recent advances in the aetiology of adolescent idiopathic scoliosis'. International Orthopaedics Vol. 32, pp. 729-734, 2008.

[10] G. Erkula, P.I.D. Sponseller, A.E. Kiter, 'Rib deformity in scoliosis'. European Spine Journal Vol. 12, pp. 281-287., 2003.

[11] B. Sevastik, B. Xiong, J. Sevastik, U. Lindgren, U. Willers, 'Rib-vertebral angle asymmetry in idiopathic, neuromuscular and experimentally induced scoliosis.'. European Spine Journal Vol. 6, pp. 84-88, 1997.

[12] D.J. Wever, A.G. Veldhuizen, J.P. Klein, P.J. Webb, G. Nijenbanning, J.C. Cool, J.R. van Horn, 'A biomechanical analysis of the vertebral and rib deformities in structural scoliosis'. European Spine Journal Vol. 8, pp. 252-260, 1999.

[13] W.J. Kane, 'Scoliosis prevalence: a call for a statement of terms.'. Clin Orthop Vol. 126, pp. 43-46, 1997.

[14] A.J. Stirling, D. Howel, P.A. Millner, S. Sadiq, D. Sharples, R.A. Dickson, 'Late-onset idiopathic scoliosis in children six to fourteen years old. A cross-sectional prevalence study'. $J$ Bone Joint Surg Vol. 78-A, pp. 1330-1336, 1996.

[15] H.-K. Wong, J.H.P. Hui, U. Rajan, H.-P. Chia, 'Idiopathic scoliosis in singapore schoolchildren: A prevalence study 15 years into the screening program.'. Spine Vol. 30, pp. 1188-1196, 2005.

[16] T. Morais, M. Bernier, F. Turcotte, 'Age- and sex-specific prevalence of scoliosis and the value of school screening programs'. Am J Public Health Vol. 75, pp. 1377-1380, 1985.

[17] E. Rogala, D. Drummond, J. Gurr, 'Scoliosis: incidence and natural history. A prospective epidemiological study'. J Bone Joint Surg Vol. 60-A, pp. 173-176, 1978.

[18] J.E. Lonstein, J.M. Carlson, 'The prediction of curve progression in untreated idiopathic scoliosis during growth.'. J Bone Joint Surg Vol. 66-A, pp. 1061-1071., 1984.

[19] S.L. Weinstein, L.A. Dolan, K.F. Spratt, K.K. Peterson, M.J. Spoonamore, I.V. Ponseti, 'Health and function of patients with untreated idiopathic scoliosis: A 50-year natural history study'. JAMA Vol. 289, pp. 559-567, 2003.

[20] S.L. Weinstein, L.A. Dolan, J.C.Y. Cheng, A. Danielsson, J.A. Morcuende, 'Adolescent idiopathic scoliosis'. Lancet Vol. 371, pp. 1527-1537, 2008.

[21] H.-R. Weiss, S. Bess, M. Wong, V. Patel, D. Goodall, E. Burger, 'Adolescent idiopathic scoliosis - to operate or not? A debate article'. Patient Safety in Surgery Vol. 2, pp. 25-38., 2008.

[22] B.V. Reamy, J.B. Slakey, 'Adolescent Idiopathic scoliosis: review and current concepts'. Am Fam Physician Vol. 64, pp. 111-116, 2001. 
[23] P.D. Sponseller, 'Bracing for adolescent idiopathic scoliosis in practice today'. Journal of pediatric orthopaedics Vol. 31, pp. S53-S60, 2011.

[24] T. Maruyama, 'Bracing adolescent idiopathic scoliosis: A systematic review of the literature of effective conservative treatment looking for end results 5 years after weaning'. Disability \& Rehabilitation Vol. 30, pp. 786-791, 2008.

[25] J.F. Aguilar Madeira, H.L. Pina, E.B. Pires, J. Monteiro, 'Surgical correction of scoliosis: Numerical analysis and optimization of the procedure'. International Journal for Numerical Methods in Biomedical Engineering Vol. 26, pp. 1087-1098, 2010.

[26] Y. Lafon, J.P. Steib, W. Skalli, 'Intraoperative three dimensional correction during in situ contouring surgery by using a numerical model'. Spine Vol. 35, pp. 453-459, 2010.

[27] Y. Lafon, V. Lafage, J. Dubousset, W. Skalli, 'Intraoperative three-dimensional correction during rod rotation technique'. Spine Vol. 34, pp. 512-519, 2009.

[28] M. Robitaille, C.É. Aubin, H. Labelle, 'Effects of alternative instrumentation strategies in adolescent idiopathic scoliosis: A biomechanical analysis'. Journal of Orthopaedic Research Vol. 27, pp. 104-113, 2009.

[29] L. Gréalou, C.É. Aubin, H. Labelle, 'Rib cage surgery for the treatment of scoliosis: a biomechanical study of correction mechanisms'. Journal of Orthopaedic Research Vol. 20, pp. 1121-1128, 2002.

[30] A. Rohlmann, T. Zander, N. Burra, G. Bergmann, 'Flexible non-fusion scoliosis correction systems reduce intervertebral rotation less than rigid implants and allow growth of the spine: a finite element analysis of different features of orthobiom ${ }^{\mathrm{TM}}{ }^{\mathrm{M}}$. European Spine Journal Vol. 17, pp. 217-223, 2008.

[31] W.J. Kim, S.H. Lee, S.W. Shin, C.H. Rivard, C. Coillard, S. Rhalmi, 'The influence of fixation rigidity on intervertebral joints. An experimental comparison between a rigid and a flexible system.'. J Korean Neurosurg Soc Vol. 37, pp. 364-369, 2005.

[32] S.J. Hall (1991) The biomechanics of the human spine. In: Basic biomechanics. 2nd edn. McGraw-Hill, Boston, USA, pp 252-293

[33] M.G. Gardner-Morse, I.A.F. Stokes, 'Structural behavior of human lumbar spinal motion segments'. J Biomech Vol. 37, pp. 205-212., 2004.

[34] F. Heuer, H. Schmidt, Z. Klezl, L. Claes, H.-J. Wilke, 'Stepwise reduction of functional spinal structures increase range of motion and change lordosis angle'. J Biomech Vol. 40, pp. 271-280., 2007.

[35] M.M. Panjabi, J.N. Hausfeld, A.A. White, 'A biomechanical study of the ligamentous stability of the thoracic spine in man'. Acta Orthop Scand Vol. 52, pp. 315, 1981.

[36] M.M. Panjabi, R.A. Brand, A.A. White, 'Three-dimensional flexibility and stiffness properties of the human thoracic spine'. J Biomech Vol. 9, pp. 185-192., 1976.

[37] J.A.A. Miller, A.B. Schultz, D.N. Warwick, D.L. Spencer, 'Mechanical properties of lumbar spine motion segments under large loads'. Journal of Biomechanics Vol. 19, pp. 79-84, 1986.

[38] M.J. Schendel, K.B. Wood, G.R. Buttermann, J.J. Lewis, J.W. Ogilvie, 'Experimental measurement of ligament force, facet force, and segment motion in the human lumbar spine'. Journal of Biomechanics Vol. 26, pp. 427-438, 1993.

[39] A. Schultz, D.N. Warwick, M.H. Berkson, A.L. Nachemson, 'Mechanical properties of human lumbar spine motion segments - Part I: responses in flexion, extension, lateral bending and torsion.'. J Biomech Eng Vol. 101, pp. 46-52, 1979.

[40] I. Busscher, J.H. van Dieen, I. Kingma, A.J. van der Veen, G.J. Verkerke, A.G. Veldhuizen, 'Biomechanical characteristics of different regions of the human spine: an in vitro study on multilevel spinal segments'. Spine Vol. 34, pp. 2858-2864, 2009.

[41] Y. Guan, N. Yoganandan, J. Moore, F.A. Pintar, J. Zhang, D.J. Maiman, P. Laud, 'Momentrotation responses of the human lumbosacral spinal column'. J Biomech Vol. 40, pp. 1975-1980, 2007.

[42] M.M. Panjabi, T.R. Oxland, I. Yamamoto, J.J. Crisco, 'Mechanical behavior of the human lumbar and lumbosacral spine as shown by three-dimensional load-displacement curves'. $J$ Bone Joint Surg Vol. 76-A, pp. 413-424, 1994. 
[43] R. Watkins, R. Watkins, L. Williams, S. Ahlbrand, R. Garcia, A. Karamanian, L. Sharp, C. Vo, T.P. Hedman, 'Stability provided by sternum and rib cage in the thoracic spine.'. Spine Vol. 30, pp. 1283-1286, 2005.

[44] S.K. Stanley, A.J. Ghanayem, L.I. Voronov, R.M. Havey, O. Paxinos, G. Carandang, M.R. Zindrick, A.G. Pathwardhan, 'Flexion-extension response of the thorocolumbar spine under compressive follower preload'. Spine Vol. 29, pp. E 510- E 514., 2004.

[45] M.J. Fagan, S. Julian, A.M. Mohsen, 'Finite element analysis in spine research'. Proceedings of the Institution of Mechanical Engineers, Part $H$ : Journal of Engineering in Medicine Vol. 216, pp. 281-298, 2002.

[46] T.B. Grivas, E. Vasiliadis, C. Mihas, O. Savvidou, 'The effect of growth on the correlation between the spinal and rib cage deformity: implications on idiopathic scoliosis pathogenesis'. Scoliosis Vol. 2, pp. 11-17, 2007.

[47] Y. Petit, C. Aubin, H. Labelle, 'Patient-specific mechanical properties of a flexible multi-body model of the scoliotic spine'. Medical and Biological Engineering and Computing Vol. 42, pp. 5560, 2004.

[48] M. Viceconti, S. Olsen, L.P. Nolte, K. Burton, 'Extracting clinically relevant data from finite element simulations'. Clin Biomech Vol. 20, pp. 451-454, 2005.

[49] I. Busscher, A.J. van der Veen, J.H. van Dieen, I. Kingma, G.J. Verkerke, A.G. Veldhuizen, 'In vitro biomechanical characteristics of the spine: A comparison between human and porcine spinal segments'. Spine Vol. 35, pp. E35-E42, 2010.

[50] H.J. Wilke, B. Jungkanz, K. Wenger, L.E. Claes, 'Spinal segment range of motion as a function of in vitro test conditions: effects of exposure period, accumulated cycles, angulardeformation rate and moisture condition.'. Anat Rec Vol. 251, pp. 15-19, 1998.

[51] T. Andriacchi, A. Schultz, T. Belytschko, J. Galante, 'A model for studies of mechanical interactions between the human spine and rib cage'. J Biomech Vol. 7, pp. 497-507, 1974.

[52] N. Arjmand, A. Shirazi-Adl, 'Role of intra-abdominal pressure in the unloading and stabilization of the human spine during static lifting tasks'. European Spine Journal Vol. 15, pp. 1265-1275, 2006.

[53] J. Cholewicki, K. Juluru, A. Radebold, M.M. Panjabi, S.M. McGill, 'Lumbar spine stability can be augmented with an abdominal belt and/or increased intra-abdominal pressure'. European Spine Journal Vol. 8, pp. 388-395, 1999.

[54] P.W. Hodges, A.E.M. Eriksson, D. Shirley, S.C. Gandevia, 'Intra-abdominal pressure increases stiffness of the lumbar spine.'. J Biomech Vol. 38, pp. 1873-1880., 2005.

[55] M. Essendrop, T.B. Andersen, B. Schibye, 'Increase in spinal stability obtained at levels of intra-abdominal pressure and back muscle activity realistic to work situations'. Applied Ergonomics Vol. 33, pp. 471-476, 2002.

[56] K. Daggfeldt, A. Thorstensson, 'The mechanics of back-extensor torque production about the lumbar spine'. J Biomech Vol. 36, pp. 815-825, 2003.

[57] D. Wever, J. Elstrodt, A. Veldhuizen, J.R.v. Horn, 'Scoliosis correction with shape-memory metal: results of an experimental study'. European Spine Journal Vol. 11, pp. 100-106, 2002.

[58] P.O. Newton, C.L. Farnsworth, V.V. Upasani, R. Chambers, S.H. Yoon, P. Firkins, 'Dual and Single Memory Rod Construct Comparison in an Animal Study'. Spine Vol. 36, pp. E904E913, 2011. 


\section{Chapter 2}

Models of the spine and trunk and their validation processes 
In this chapter the final model of the adolescent trunk (figure 1) and the models used in the various studies and for validation are presented in detail, since only general descriptions of the models are given in each of the subsequent chapters. First the structure of the model is explained and compared to the anatomy, followed by a section describing the various analyses that can be carried out with these models. Then the validation process of each of the models is described. In the discussion, the assumptions of the modelling and the influence of these assumptions on the validity of the models are discussed, resulting in a conclusion on the usability of the model.

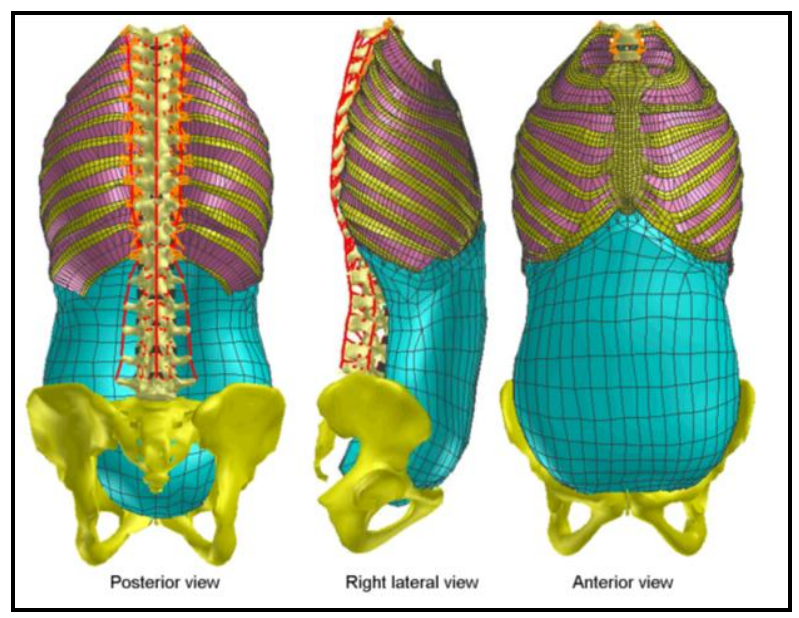

Figure 1: Finite element model of adolescent spine and trunk.

\section{$2.1 \quad$ Structure of the model}

In this section the structure of the model is explained and a comparison to the anatomy is made, assumptions and reasoning for the approach are discussed, and the used parameters are summarized. First the modelling of the spine is discussed: which is subdivided into the parametric model of the spine geometry, material properties, differences between adult and adolescent spine and finally the scoliotic spine. Then follows the modelling of the (adolescent) ribcage and intra-abdominal pressure, and this section ends with a description of the development of the adolescent, scoliotic trunk model.

\subsubsection{The spine}

\subsubsection{Parametric model of the spine geometry}

The parametric model of the spine is build using an in-house written matlab routine. The motion segment is the smallest unit representing the mechanical behaviour of the spine, and the spine is in essence a repetition of these motion segments. When the model has to represent a larger part of the spine, the routine for the motion segment is repeated as often as necessary, in which the lower vertebra of the new 
motion segment coincides with the upper vertebra of the previous motion segment, resulting in a continuous spine model (vertebra-disc-vertebra-disc etcetera).

The used motion segment structure, containing two vertebrae, an intervertebral disc, seven spinal ligaments and two facet joints is shown in figure 2.

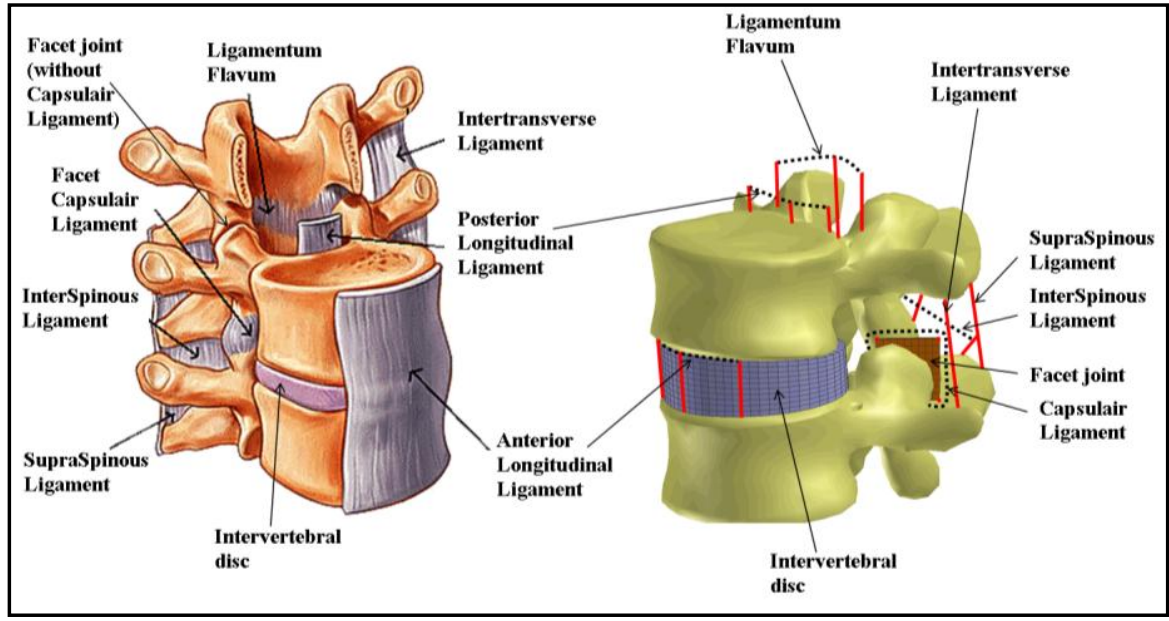

Figure 2: Lumbar motion segment: two adjacent vertebrae including the facet joints, the intervertebral disc and seven spinal ligaments.

Left: anatomy, right: the model.

The parametric model is represented in figure 3 and an overview of the used parameters is given in table 1. Detailed quantitative data per vertebral level was taken from literature (see appendix A).

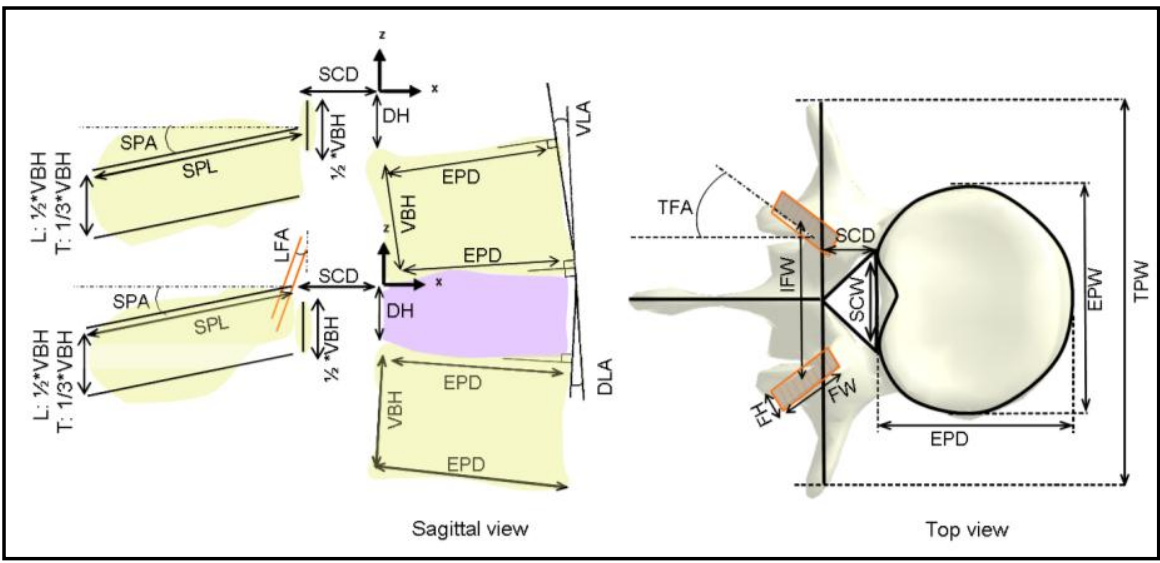

Figure 3: Parameters for defining the geometry of a motion segment. Definition of abbreviations and additional information is given in table 1. Note the different height for the spinous process in the lumbar $(L)$ and Thoracic $(T)$ region. 


\begin{tabular}{|cllc|}
\hline Abbreviation & Parameter & Notes & Fig. \\
\hline EPD & EndPlate Depth & Largest distance & $3 \mathrm{a} \& \mathrm{~b}$ \\
EPW & EndPlate Width & Largest distance & $3 \mathrm{~b}$ \\
TPW & Transverse Process Width & Largest distance & $3 \mathrm{~b}$ \\
SPL & Spinous Process Length & Diagonal distance & $3 \mathrm{a}$ \\
SPA & Spinous Process Angle & Regard to local horizontal axis & $3 \mathrm{a}$ \\
VBH & Vertebral Body Height & Posterior height & $3 \mathrm{a}$ \\
VLA & Vertebra Lordosis Angle & & $3 \mathrm{a}$ \\
DH & Disc Height & Posterior height & $3 \mathrm{a}$ \\
DLA & Disc Lordosis Angle & & $3 \mathrm{a}$ \\
LFA & Longitudinal Facet Angle & Regard to local vertical axis & $3 \mathrm{a}$ \\
TFA & Transverse Facet Angle & Regard to local horizontal axis & $3 \mathrm{~b}$ \\
IFW & Inter Facet Width & Horizontal distance & $3 \mathrm{~b}$ \\
FW & Facet Width & & $3 \mathrm{~b}$ \\
FH & Facet Height & & $3 \mathrm{~b}$ \\
SCD & Spinal Canal Depth & Largest distance & $3 \mathrm{~b}$ \\
SCW & Spinal Canal Width & Largest distance & $3 \mathrm{~b}$ \\
\hline
\end{tabular}

Table 1: Parameters used to define the geometry of a motion segment.

To define the geometry of a motion segment, first the shape and position of four endplates are defined. These will provide the outline of the disc and the vertebral bodies. All other structures are related to the position and orientation of these four endplates, using a local coordinate frame with the origin defined in the most posterior point (mid sagittal plane) of the lower endplate of the upper vertebra (see figure 3). Please note that this origin is only used for building up the parametric model and not for motion analysis; the local coordinate system that is used for the motion analysis and the definition of the planes is defined in Appendix B.

The geometry of the spinous process and transverse process is simplified to a $2 \mathrm{D}$ representation: for the spinous process only the length, orientation and height are modelled and for the transverse process only the width and height are considered and a mediolateral orientation is assumed (figure 3).

For the spinal canal, a triangular shape is assumed combined with the defined depth and width.

The facet joints are represented by four surfaces (left inferior articulating with left superior, right inferior articulating with right superior). The capsular ligament connects the inferior and superior surfaces of the facet joint at the four corners. The size of the rectangular surface is defined by the height and width, the orientation by the longitudinal and transverse orientation (relative to disc orientation). The distance between the inferior and posterior facet surfaces is $0.6 \mathrm{~mm}{ }^{1}$ and the distance between the midpoint of the left and right facet joint is the inter facet width (figure 3).

Thus far, the geometry of the outline of the vertebrae, the disc and the facet joints are defined. A further definition of the shape of the endplates, the division of the disc into a nucleus and an annulus and the precise attachment points of the ligaments is needed. For this, a number of assumptions are made.

For the shape of the endplate in the transverse plane, a kidney shape was assumed and a general equation of its closed contour was defined ${ }^{2}$ and parameters for the 
various regions of the spine were defined by Langrana et al. ${ }^{3}$. The equation and parameters are given in appendix $C$. These equations provide the shape of the endplate, which is scaled in the anterior-posterior direction to get the correct depth and in the mediolateral direction to give the correct width.

In the sagittal plane, the endplate has a curvature, which is also modelled (see appendix $\mathrm{C}$ ). The shape of the nucleus in the transverse plane is taken similar to the outline of the endplate. The size of the nucleus is set at $43 \%$ of the total disc area in the midsagittal plane ${ }^{4}$.

The attachment points of the ligaments are shown in figure 4. For the thoracic and lumbar region, other orientations for the interspinal ligament are reported in literature ${ }^{5}$, which are both presented in figure 4.

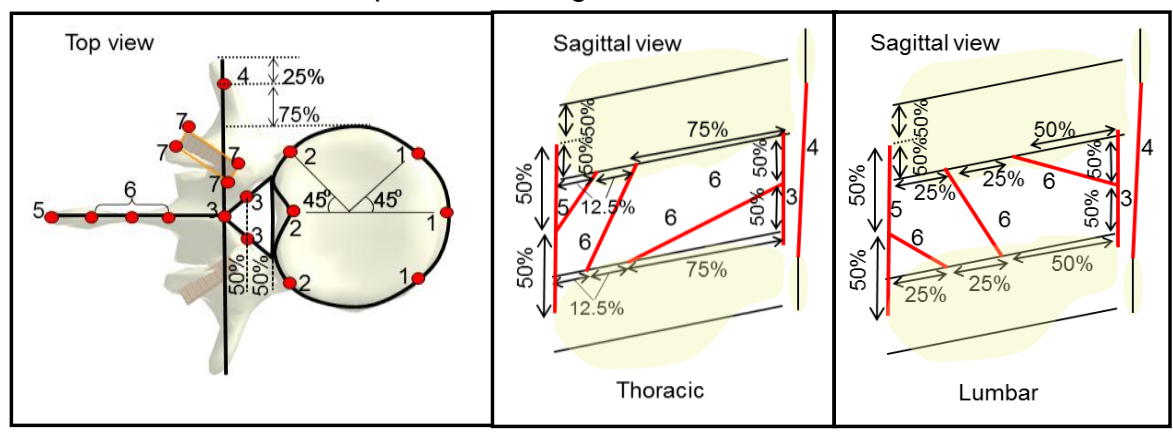

Figure 4: Definition of the attachment points of the ligaments.

Numbering of the ligaments: 1=Anterior Longitudinal Ligament, 2=Posterior Longitudinal Ligament, 3=Ligamentum Flavum, 4=Inter Transverse Ligament, 5=Supra Spinal Ligament, 6=Inter Spinal Ligament, 7=Capsular Ligament.

The interspinal ligament was modelled with three elements to capture different fibre orientations. Since the middle element represents the major orientation ${ }^{6}, 50 \%$ of the total cross-sectional area is attributed to this element, and $25 \%$ to each of the other elements. The anterior longitudinal ligament, posterior longitudinal ligament, ligamentum flavum and capsular ligament were modelled with multiple elements of equal cross-sectional area to capture the mechanical effect of their width. An overview of the cross-sectional areas of the ligaments for the various regions of the spine is presented in table 2.

\begin{tabular}{|rcccc|} 
Ligament & $\begin{array}{c}\text { Abbre- } \\
\text { viation }\end{array}$ & $\begin{array}{c}\text { Lumbar Area } \\
{\left[\mathbf{m m}^{\mathbf{2}}\right]}\end{array}$ & $\begin{array}{c}\text { Thoracic Area } \\
{\left[\mathbf{m m}^{\mathbf{2}}\right]}\end{array}$ & Ref. \\
\hline Anterior Longitudinal Ligament & ALL & 66 & 31 & 7 \\
Posterior Longitudinal Ligament & PLL & 29 & 18 & 7 \\
Ligamentum Flavum & LF & 39 & 27 & 7 \\
Inter Transverse Ligament & ISL & 40 & 30 & 8 \\
Supra Spinal Ligament & SSL & 30 & 10 & 7 \\
Inter Spinal Ligament & ITL & 2 & 2 & 8 \\
Capsular Ligament & CL & 30 & 30 & 8 \\
\hline
\end{tabular}

Table 2: Cross-sectional areas of the ligaments. 


\subsubsection{Material properties}

In table 3, an overview of the chosen element types and material parameters is presented.

Since the vertebrae are defined as rigid bodies, no material parameters need to be defined for these elements. In the intervertebral disc, the nucleus pulposes and annulus fibrosus have different mechanical properties. The nucleus is modelled as an incompressible gel, while the annulus is modelled as an isotropic matrix containing circumferential tension only fibres (volume ratio $=16 \%$ ) ${ }^{9}$ oriented at $+30^{\circ}$ and $-30^{\circ}$ to the transverse plane ${ }^{10}$.

Since an element cannot have two different fibre orientations, each annulus element is represented twice at the same position, using the same nodes. In this way the two fibre orientation can be modelled. The represented material properties in table 3 are for the total annulus representation, with each layer contributing half of the stiffness of the total matrix.

\begin{tabular}{|rlll|}
\hline & Element type & Mechanical properties & Ref. \\
\hline Vertebrae & Rigid bodies & - & - \\
Nucleus & 8 node solid & $\mathrm{E}=1 \mathrm{MPa}, \boldsymbol{v}=0.495$ & 11 \\
Annulus \{ & 8 node solid & $\mathrm{E}=2 \mathrm{MPa}, \boldsymbol{v}=0.45$ & 12 \\
Ligaments & Tension-only fibres & E $=450 \mathrm{MPa}$ & 8 \\
Facet surfaces & 4 node shell & See table 5 & - \\
\hline
\end{tabular}

Table 3: Overview of used element types and mechanical properties for the spine.

For the nucleus and annulus, SRI-elements (selective reduced integration) were used, because volumetric locking results in an overestimation of the stiffness of incompressible materials when "normal" elements are used (the bulk modulus becomes infinite large, when the Poisson's ratio approaches 0.5). These SRIelements only use one integration point for the volumetric strain, while eight integration points are used for the deviatoric strain, hereby avoiding overestimation of the stiffness due to the large bulk modulus.

The ligaments are modelled as tension-only bars. The non-linear stress-strain behaviour of the ligaments was implemented by combining three linear regions with different E-moduli for certain strain intervals. Since the length of the ligaments influences the tension stiffness, the discrepancy between the length in the model and experimental data will result in a difference in stiffness. To prevent this effect, the reported experimental stiffness $(\mathrm{k})$, rather than the reported $\mathrm{E}$-modulus, is used from literature. The corresponding E-modulus is than calculated, using the area and length of the (modelled) ligament. The reported pre-strain for ligaments was also modelled, and was set to $10 \%$ of the maximum strain of the first interval for each of the ligaments. An overview of the ligament properties is presented in table 4. 


\begin{tabular}{|c|c|c|c|c|c|c|c|}
\hline \multirow[b]{2}{*}{$\begin{array}{l}\text { Liga- } \\
\text { ment }\end{array}$} & \multicolumn{2}{|c|}{$I^{\text {st }}$ region } & \multicolumn{2}{|c|}{$2^{\text {nd }}$ region } & \multicolumn{2}{|c|}{$3^{\text {rd }}$ region } & \multirow[b]{2}{*}{$\begin{array}{c}\text { Pretension } \\
{[\text { [N] }}\end{array}$} \\
\hline & Strain [\%] & $\begin{array}{c}\text { E-modulus } \\
\text { [MPa] }\end{array}$ & $\begin{array}{c}\text { Strain } \\
{[\%]}\end{array}$ & $\begin{array}{c}\text { E-modulus } \\
{[\mathrm{MPa}]}\end{array}$ & $\begin{array}{c}\text { Strain } \\
{[\%]}\end{array}$ & $\begin{array}{c}\text { E-modulus } \\
\text { [MPa] }\end{array}$ & \\
\hline $\mathbf{A L L}$ & $0-12 \%^{7}$ & $9.8^{7}$ & $12-45 \%$ & $24.3^{7}$ & $45-58 \%$ & $12.4^{7}$ & 7.7 \\
\hline PLL & $0-9 \%^{7}$ & $17.4^{7}$ & $9-34 \%^{7}$ & $40.7^{7}$ & $34-45 \%^{7}$ & $13.3^{7}$ & 4.1 \\
\hline $\mathbf{L F}$ & $0-5 \%^{7}$ & $15^{8}$ & $5-50 \%^{7}$ & $19.5^{8}$ & $50-58 \%^{7}$ & $\left.6.0^{*}\right)$ & 2.9 \\
\hline ISL & $0-12 \%^{7}$ & $8.2^{7}$ & $12-30 \%^{7}$ & $18.4^{7}$ & $30-40 \%^{7}$ & $8.7^{7}$ & 3.9 \\
\hline SSL & $0-12 \%^{7}$ & $14.6^{7}$ & $12-30 \%^{7}$ & $33.1^{7}$ & $30-40 \%^{7}$ & $15.6^{7}$ & 5.3 \\
\hline ITL & $0-9 \%^{7}$ & $164^{7}$ & $9-15 \%^{7}$ & $814^{7}$ & $15-17 \%^{7}$ & $270^{7}$ & 0.2 \\
\hline CL & $0-100 \%{ }^{13}$ & $0.4^{13}$ & $\left.100-200 \%{ }^{*}\right)$ & $\left.0.9^{*}\right)$ & $\left.200-300 \%{ }^{*}\right)$ & $0.3^{*)}$ & 1.0 \\
\hline
\end{tabular}

Table 4: Mechanical properties of the spinal ligaments. Properties for CL and ITL given per side. The pretension is based on the lumbar ligament areas, and for the ISL pretension is only present in the middle element.

It is assumed that the mechanical properties of the ligaments for the various regions of the spine alter due to the difference in cross-sectional area, length and attachment points, rather than the changing E-modulus. We thus used the same E-modulus throughout the spine.

For the facet joints, a non-linear contact definition is used to represent the non-linear cartilage behaviour (see table 5). Furthermore, a friction coefficient of 0.01 was implied.

\begin{tabular}{|cc|}
$\begin{array}{c}\text { Compression } \\
{[\mathbf{m m}]}\end{array}$ & $\begin{array}{c}\text { Stiffness } \\
{[\mathbf{N} / \mathbf{m}]}\end{array}$ \\
\hline $0.0-0.25$ & 0.1 \\
$0.25-0.5$ & 0.5 \\
$0.5-1.0$ & 4.2 \\
$1.0-1.5$ & 7.5 \\
$1.5-2.0$ & 22.5 \\
$>2.0$ & 90.0 \\
\hline
\end{tabular}

Table 5: Non-linear cartilage behaviour of facet joints

\subsubsection{Differences between adult and adolescent spine}

The main goal of this thesis is to present a numerical model of an average adolescent spine that quantifies the various biomechanical aspects that are important in scoliosis correction. However, most data in literature that are relevant for both the input and validation of the model are for adults. Therefore, first an adult model of the spine has been developed and validated.

Then, an adolescent geometry has been developed, by local scaling of the adult parametric model, as previously described for a paediatric model ${ }^{14}$. As severe scoliosis mainly occurs in girls, we used average geometrical growth data for girls between 10 year and maturity for this scaling. Both the growth change (between 
10 year and maturity) and the scaling for the model (from adult to 10 year) are presented in table 6.

As literature data was not available for transverse and spinous process growth, we assumed this growth to be related to the depth growth of the vertebrae; both grow through enchondral ossification combined with periosteal growth. Growth changes in vertebral and disc lordosis angle are assumed to be zero, because the change in lumbar lordosis during growth is less than $1^{\circ}$ per motion segment ${ }^{15-17}$.

Unfortunately, no data on adolescent material properties are available. It is therefore assumed that the adult material parameters are also representative for adolescents.

\begin{tabular}{|c|c|c|c|}
\hline Parameter & $\begin{array}{l}\text { Growth change: } \\
\text { 10year } \rightarrow \text { adult }\end{array}$ & $\begin{array}{l}\text { Scaling model: } \\
\text { adult } \rightarrow 10 \text { year }\end{array}$ & Ref. \\
\hline vertebral body height & $+41.0 \%$ & $71 \%$ & 18 \\
\hline disc height & $+3.3 \%$ & $97 \%$ & 19 \\
\hline endplate width & $+10.0 \%$ & $91 \%$ & 18 \\
\hline endplate depth & $+25.5 \%$ & $80 \%$ & 20 \\
\hline nucleus size & $-14.0 \%$ & $116 \%$ & 21,4 \\
\hline transverse processes width & $+25.5 \%$ & $80 \%$ & - \\
\hline spinous process length & $+25.5 \%$ & $80 \%$ & - \\
\hline ligament area & $+18.3 \%$ & $85 \%$ & 22 \\
\hline facet height & $+28.0 \%$ & $78 \%$ & 23 \\
\hline facet width & $+38.0 \%$ & $72 \%$ & 23 \\
\hline spinal canal depth & $+0.0 \%$ & $100 \%$ & 24 \\
\hline spinal canal width & $+0.0 \%$ & $100 \%$ & 24 \\
\hline inter facet width & $+0.0 \%$ & $100 \%$ & 25 \\
\hline transverse facet angle & $+0.0 \%$ & $100 \%$ & 25 \\
\hline longitudinal facet angle & $+0.0 \%$ & $100 \%$ & 25 \\
\hline spinous process angle & $+0.0 \%$ & $100 \%$ & 25 \\
\hline vertebra lordosis angle & $+0.0 \%$ & $100 \%$ & - \\
\hline disc lordosis angle & $+0.0 \%$ & $100 \%$ & - \\
\hline
\end{tabular}

Table 6: Geometrical change during growth spurt (10 year-adult), as reported for girls and scaling of the model from adult to ten year

\subsubsection{Scoliotic spine}

The scoliotic model is not patient-specific, but a representation of an average adolescent spine with scoliosis. It had to be representative for the targeted patients of the new scoliosis correction implant, a group similar to the group currently treated with a brace: a moderate but progressive scoliosis (25- $45^{\circ}$ Cobb angle).

The most common types of scoliosis are single thoracic and double thoracolumbar curves $^{26-30}$. The correction of a double curve would be, in essential, like correcting two single curves. Therefore we will focus on the single thoracic curve in this thesis. The most frequent location of the apex in a single thoracic curve is T8 ${ }^{31}$. 
The precise relation between the deformity in the frontal plane and the axial rotation is not clear, and is likely patient-specific, but it has been suggested that progressive

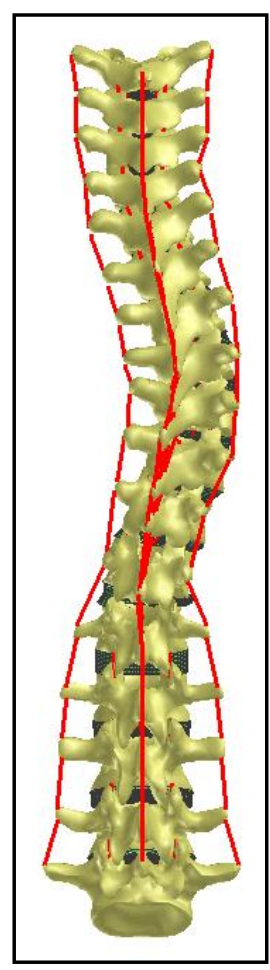
curves have more axial rotation, because the axial rotation increases the lateral deformity ${ }^{32}$.

Based on these data, the scoliotic model was provided with a single thoracic curve, with the apex at T8. The Cobb angle between the two most tilted vertebrae (T6 and T10) was set to $32^{\circ}$ and the axial rotation of the apex was $24^{\circ}$ (figure 5).

The scoliosis is created by prescribing a lateral translation and axial rotation at the vertebrae inside the scoliotic curve (T4T11), while the lowermost vertebra (L5) was fully fixed and the uppermost vertebra (T1) was fixed for translations in the transverse plane.

In the model it is assumed that the vertebrae themselves are not deformed and therefore all wedging and axial rotation is located in the intervertebral discs. In this deformed position all stresses and strains are reset to zero, so the scoliotic position is the new "neutral position" of the scoliotic model. Subsequently, a similar pretension is applied to the spinal ligaments as in the healthy situation (table 4).

Figure 5: Posterior view of scoliotic spine model. The Cobb-angle between T6-T10 is 32 degrees and the axial rotation of the apex (T8) is 24 degrees.

\subsubsection{Ribcage}

The basic anatomy of the ribcage is based on a multi-body model of a $5^{\text {th }}$ percentile female, developed by the ESI Group. This is a so-called human articulated rigid body (HARB) model, meaning that the bones of the human body are represented by rigid bodies, which can articulate with one other and are connected by joints that allow movement. Ligaments, muscles and organs are also represented. Some of the rigid bodies can be replaced by deformable finite element parts, such as the ribs. This $5^{\text {th }}$ percentile female model is chosen, since the geometry compares well with that of a ten year old girl: width, height and length from the model are compared to reported data for 10 year old girls ${ }^{33}$ (see table 7 ). It is reported in literature that the shape of the ribcage in the transverse plane is already comparable to those of adults, at the age of two year ${ }^{34}$, and the rib-vertebral angle does not change during adolescence ${ }^{34}$. This makes the geometry of the ribcage model representative for adolescent girls. 
Measured parameter

Model $\mathbf{5}^{\text {th }}$ percentile female

Measurement 10 year old girls

Anterio-posterior diameter

Mediolateral diameter

$14.8 \mathrm{~cm}$

$14.2 \mathrm{~cm}$

T1-T12 height

$21.2 \mathrm{~cm}$

$20.7 \mathrm{~cm}$

$22.8 \mathrm{~cm}$

$20.7 \mathrm{~cm}$

Table 7: Comparison between 5th percentile female model and measurements in 10 year-old girls

A detailed description of the total $5^{\text {th }}$ percentile female human model is given in a modelling study by $\mathrm{Na}$ et al. ${ }^{35}$ in this thesis, only the ribcage will be described.

At the posterior side, most ribs articulate with the vertebral bodies of the vertebrae above and below (2 costovertebral joints per rib) and with the transverse processes of the vertebra below (1 costotransverse joint per rib). Due to the different shape of the $11^{\text {th }}$ and $12^{\text {th }}$ rib (floating ribs), the costotransverse contacts do not exist for the T11 and T12. These floating ribs also only articulate with one vertebral body; that of the lower vertebrae. The first rib also only articulates with the lower vertebrae, the T1. The model correctly represents this anatomical situation.

All contacts are modelled as non-linear penalty contacts.

The ribs are attached to the spine by costovertebral ligaments (between the rib head and the vertebral bodies of the lower and upper vertebrae) and costotransverse ligaments (between the neck of the rib and the transverse processes above and below). The costovertebral ligaments are the interosseous costovertebral ligament and the radiate costovertebral ligament. The radiate costovertebral ligament consists of three bands, the superior and inferior attaches the rib to the adjacent vertebral bodies, while the intermediate connects the rib to the intervertebral disc. The costotransverse ligaments are the posterior costotransverse ligament, the superior costotransverse ligament and the interosseous costotransverse ligament. A comparison between the modelling and anatomy of the ligaments and joints is made in figure 6.

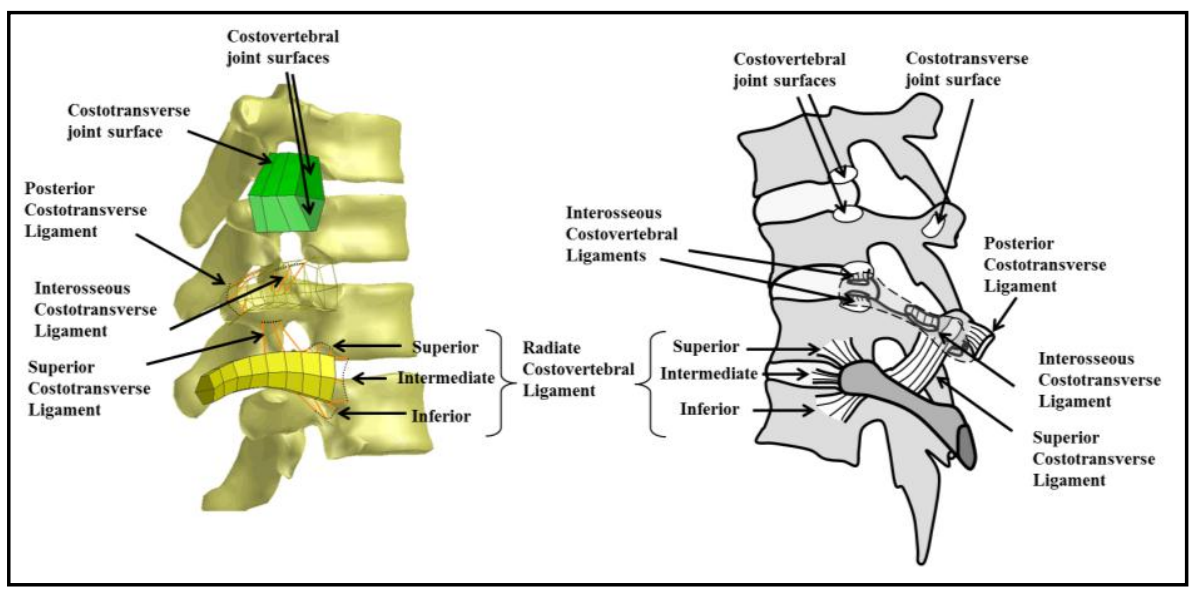

Figure 6: Comparison between model (left) and anatomy ${ }^{36}$ (right) of the costovertebral and costotransverse joints and ligaments 
Most important difference between the model and anatomy is that the contact surfaces of the vertebrae are modelled as flat surfaces. The true anatomy of these surfaces is curved in 3D. However, the contact definitions did not allow for a 3D curved surface in combination with non-linear penalty contact. For the mechanical behaviour, the non-linearity of the contact definition is considered more important than the 3D curvature of the joint surfaces. Therefore flat surfaces are used.

Furthermore, in the model, the costotransverse and both costovertebral contacts articulating with one rib are part of the rigid body of the vertebra below this rib, while in reality the costovertebral contact articulates with both the lower and upper vertebra. Another difference is that the interosseous costovertebral ligament is not modelled. The mechanical effect of this ligament is considered very similar to that of the radiate ligament, which also attaches the rib head to the vertebral bodies.

At the anterior side, the ribs are connected to the sternum with a cartilage connection. The first five ribs each have their own costal cartilage connection (true ribs), the $6^{\text {th }}$ to the $10^{\text {th }}$ ribs use one combined cartilage connection (false ribs) and the $11^{\text {th }}$ and $12^{\text {th }}$ ribs do not connect to the sternum at all (floating ribs). In between the ribs, intercostal muscles are modelled. An anterior view of the ribcage is given in figure 7.

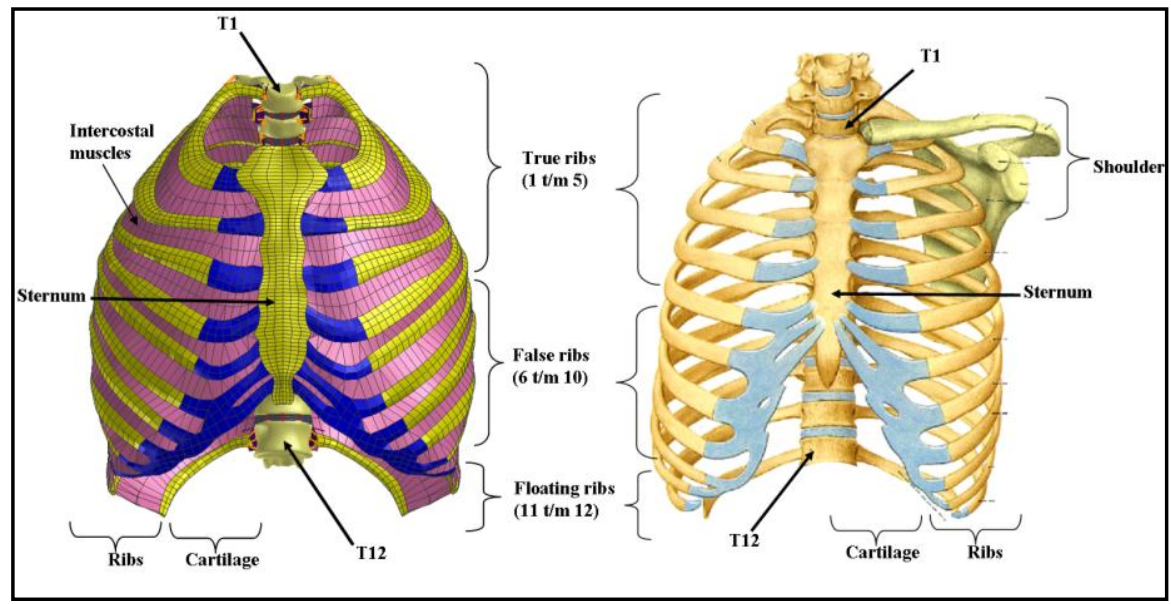

Figure 7: Comparison between model (left) and anatomy ${ }^{37}$ (right) of the ribcage and thoracic spine. The shoulder and clavicula are not present in the model. The intercostal muscles are not shown in the right figure.

An overview of the used element types and mechanical properties for the ribcage is given in table 8 . The mechanical properties of the ribs were based on experimental data for the posterior part of the ribs ${ }^{38}$.

The intercostal muscles were modelled as a membrane reinforced by fibres, representing the main orientation of the muscle. The internal and external parts of the muscle are oriented differently. Therefore, the orientation of the fibres in the outer layer is 30 degrees with respect to the ribs, in the internal layer this orientation is 120 degrees $^{39}$. 
For muscles, the only reported mechanical property is the maximum stress, which is reported to be between 0.4 and $0.65 \mathrm{MPa}$ for trunk muscles ${ }^{40}$; an average value of $0.5 \mathrm{MPa}$ is assumed for the intercostal muscles. To determine the $\mathrm{E}$-modulus of the fibres, a strain of $0.5 \%$ is assumed in combination with $25 \%$ of the maximum stress, resulting in an E-modulus of $25 \mathrm{MPa}$ for the fibres. For the matrix, the strain is assumed to be $1 \%$ and the stress is set at $10 \%$ of the maximum level, resulting in an E-modulus of $5 \mathrm{MPa}$.

\begin{tabular}{|rlll|} 
Structure & Element type & Mechanical properties & Ref. \\
Ribs & 8 node solid & $\mathrm{E}=11 \mathrm{GPa}, v=0.3$ & 38 \\
Costal cartilage & 8 node solid & $\mathrm{E}=25 \mathrm{MPa}, \boldsymbol{v}=0.45$ & 41 \\
Intercostal muscle $\boldsymbol{\{}$ & 4 node membrane & $\mathrm{E}=5 \mathrm{MPa}, \boldsymbol{v}=0.4$ & - \\
Costovertebral \& & Tension-only fibres & $\mathrm{E}=25 \mathrm{MPa}, \boldsymbol{v}=0.3$ & - \\
consion-only bars & See table 9 & - \\
Costovertebral \& & 4 node shell & Non-linear penalty & - \\
costotransverse contacts & & contact & \\
\hline
\end{tabular}

Table 8: Overview of used element types and mechanical properties for the ribcage

Mechanical properties of the costotransverse and costovertebral ligaments have never been measured, as noted in previous modelling studies ${ }^{42-44}$. We assumed the Young's modulus of these ligaments to increase gradually from that of elastin $(E=0.6$ $\mathrm{MPa})^{45}$ to that of collagen $(\mathrm{E}=1 \mathrm{GPa})^{45}$, for seven strain regions (see table 9 ).

\begin{tabular}{|cc|}
$\begin{array}{c}\text { Strain } \\
{[\%]}\end{array}$ & $\begin{array}{c}\text { E-modulus } \\
{[\mathbf{M P a}]}\end{array}$ \\
\hline $0.0-0.001$ & 0.6 \\
$0.001-0.01$ & 1.2 \\
$0.0-0.05$ & 2.5 \\
$0.05-0.1$ & 50 \\
$0.1-0.5$ & 100 \\
$0.5-1.0$ & 200 \\
$>1.0$ & 1000 \\
\hline
\end{tabular}

Table 9: Mechanical properties of costovertebral and costotransverse ligaments

The low-strain behaviour of the costovertebral ligaments is comparable to the spinal ligaments. This is also the physiological region, since the much stiffer high strain behaviour is not reached during normal loading conditions.

Cross-sectional areas $\left(10 \mathrm{~mm}^{2}\right)$ for all costovertebral and costotransverse ligaments are based on reported data for the costotransverse ligaments ${ }^{36,46-48}$.

\subsubsection{Intra-abdominal pressure}

The intra-abdominal pressure is the pressure caused by the presence of internal organs and other soft tissues in the abdominal cavity and the passive stiffness of the abdominal and dorsal muscles. It is well known that these combined effects increase the stiffness and stability of the trunk. As measurements on the effect of the intra- 
abdominal pressure (IAP) are almost impossible without increasing the activity of the abdominal and dorsal muscles, the discussion on the precise mechanism of the IAP is still ongoing. It has been proposed that an extensor moment is generated because the IAP exerts a force down on the pelvic floor and up on the diaphragm. In combination with the flexor moment of the abdominal muscles, this will increase the trunk stiffness and stability ${ }^{49}$. Another theory is that the pressure in the abdominal cavity limits intervertebral rotation and translation and thus increases the stiffness and stability of the trunk ${ }^{50}$. It is also suggested that the IAP prevents the abdominal muscles from shortening, and thus helps maintaining the hoop-like geometry of the muscles, necessary for providing tension ${ }^{51}$. As no specific mechanism is proven right or wrong yet, all these effects should be considered when modelling the IAP. However, currently, the IAP is often modelled as a single force on the middle of the diaphragm ${ }^{52-54}$. This is most likely an oversimplification of the biomechanical function, since two effects are neglected in this way of modelling: both the increased stiffness of the abdominal muscles and the physiological function of the IAP in maintaining the shape of these muscles and the direct effects of the IAP on limiting the movement of the spinal column are neglected. A study by Gatton et al. ${ }^{55}$ showed that when the posture of the spine is changing, neglecting the elliptical shape of the abdominal muscles can result in erroneous estimations of the moments applied by these muscles (differences up to $100 \%$ for axial rotation and $37 \%$ for extension). In a recently published model, the intra-abdominal pressure is represented by a pressure vessel ${ }^{56}$, also modelling this shape change of the abdominal muscles. Main limitation of this modelling study is that only the lumbar spine is considered, neglecting the mechanical effects of the thoracic spine and ribcage, which are directly influencing the behaviour of the intra-abdominal pressure.

In the current model, the intra-abdominal pressure is modelled as an incompressible volume in the shape of the intra-abdominal cavity, with an overpressure of $1 \mathrm{kPa}$, representing a neutral standing position ${ }^{57-59}$. The total model including the thoracolumbar spine, the ribcage and IAP is shown in figure 8.

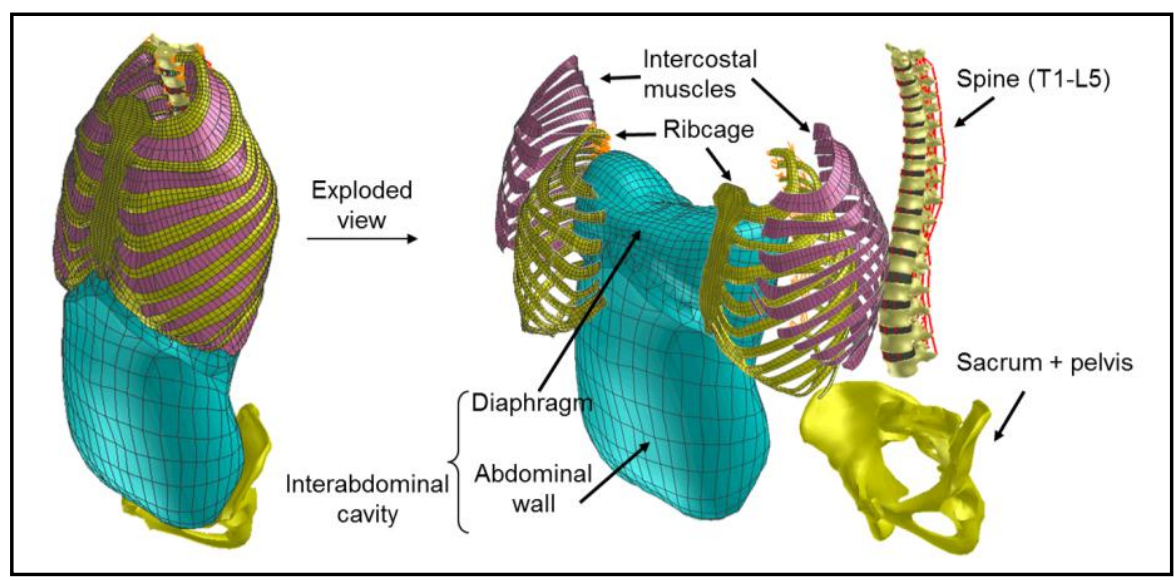

Figure 8: Anterior-lateral view of the trunk model, including the thoracolumbar spine, the ribcage, intercostal muscles, sacrum, pelvis and intra-abdominal cavity 
The shape of the intra-abdominal cavity is based on the $5^{\text {th }}$ percentile female human model (see section 2.1.2 for description of this model) and consists of two parts. The upper part of the intra-abdominal cavity is the diaphragm, and the lower part is the abdominal wall. The shape of the abdominal wall is based on the outer skin layer of the abdomen and the pelvic outline, both with an offset of $10 \mathrm{~mm}$, the estimated thickness of the skin (including the subcutaneous tissues) ${ }^{60,61}$. On the posterior side the intra-abdominal cavity is curved along the anterior side of the spine.

The membrane layer surrounding the intra-abdominal cavity interacts with both the spine (nodes implemented in rigid bodies of T10 to L4) and the ribcage (contact between diaphragm and ribs). A fibre reinforced material is used for the abdominal part of the intra-abdominal cavity to represent the orientation of the abdominal muscles. The most important muscles controlling the intra-abdominal pressure are internal oblique and transversus abdominus ${ }^{62,63}$, which were represented by fibres in the layer surrounding the intra-abdominal cavity, oriented at respectively 80 degrees and 10 degrees with the horizontal. There are no fibres in the diaphragm.

In this way of modelling, the direct effect of the IAP on the vertebrae is included, as well as the upward force of the diaphragm on the ribcage and the (passive) stiffness of the abdominal and dorsal muscles.

Again, like for the intercostal muscles, no mechanical properties, except the maximum stress, are reported for the diaphragm and the abdominal muscles. The maximum stress is reported to be between 0.4 and $0.65 \mathrm{MPa}$ for trunk muscles ${ }^{40}$; for both muscles, an average value of $0.5 \mathrm{MPa}$ is assumed. For the diaphragm a strain of $0.5 \%$ is assumed in combination with $35 \%$ of the maximum stress, resulting in an E-modulus of $35 \mathrm{MPa}$. For the matrix of the abdominal muscles, the strain is assumed to be $1 \%$ and the stress is set at $10 \%$ of the maximum level, resulting in an E-modulus of $5 \mathrm{MPa}$. For the fibres of the abdominal muscles, the strain is assumed to be $0.5 \%$ and the stress is set at $15 \%$ of the maximum level, resulting in an $\mathrm{E}$ modulus of $15 \mathrm{MPa}$.

An overview of the element types and mechanical properties of the intra-abdominal cavity is given in table 10 .

\begin{tabular}{|rll|}
\hline Structure & Element type & $\begin{array}{l}\text { Mechanical } \\
\text { properties }\end{array}$ \\
\hline Diaphragm & $\begin{array}{l}\text { 4 node membrane } \\
\text { 4 node membrane }\end{array}$ & $\mathrm{E}=35 \mathrm{MPa}, v=0.3$ \\
Abdominal wall $\{=5 \mathrm{MPa}, v=0.45$ \\
tension-only fibres & $\mathrm{E}=15 \mathrm{MPa}, v=0.3$ \\
\hline
\end{tabular}

Table 10: Overview of used element types and mechanical properties for the intra-abdominal cavity 


\subsubsection{Scoliotic trunk model}

As described in section 2.1.1.4, a scoliotic model of the spine has been developed by prescribing a lateral translation and axial rotation at the vertebrae. As we modelled the complete trunk, these displacements of the vertebrae resulted in a natural deformation of the entire trunk (see figure 9).

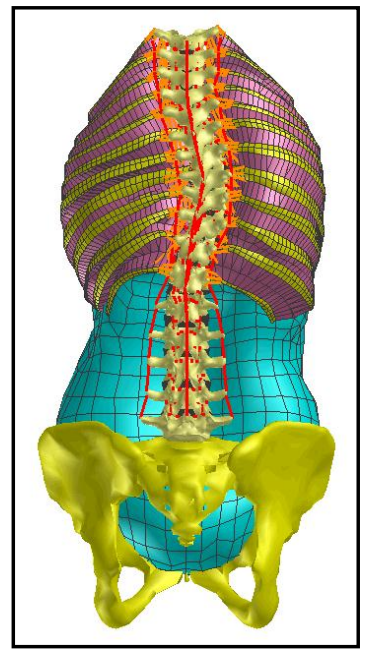

Figure 9: Scoliotic trunk model in posterior view. The Cobb-angle between T6-T10 is 32 degrees and the axial rotation of the apex (T8) is 24 degrees.

\subsection{Methods}

The FE models, as described in the previous section, can be used to optimize the design of a scoliosis correction implant and improve general knowledge about the biomechanics of the spine. Various analyses are conducted in this thesis; the methods and their use are briefly discussed in this chapter.

\subsubsection{Stiffness}

Applying known loading conditions will result in rotations and translations of the vertebrae. This can be used to determine the stiffness for various models or for the same model for various loading conditions.

In the presented studies, the rotations of the vertebrae are determined by analysing the movement of the local coordinate frame of the vertebrae (see appendix B). This is used in chapter 3 to determine the influence of changing geometry during growth on spinal stiffness, in chapter 4 to determine the influence of inter-personal geometry changes on spinal stiffness, in chapter 5 to determine the influence of the posterior 3 $\mathrm{cm}$ of the ribs on the spinal stiffness, and in chapter 6 to determine the influence of the spine, the ribcage and intra-abdominal pressure on the stiffness of the adolescent trunk. 
Last but not least, in chapter 7 this is used to predict the effect of the implant on the geometry of the spine and trunk. Both the induction of scoliosis in a healthy spine (as conducted in animal experiments) and correction of a scoliotic spine (the goal of the implant) are analysed.

\subsubsection{Forces in ligaments and muscles}

In all bar elements (like the ligaments and muscles), the elongation and force can be determined. This is especially useful when comparing the ligament and muscle forces for various conditions. Although the current model contains a limited number of muscles, when expanding the number of muscles in future models, this analysis can be used to compare the effect of scoliosis on the muscle activity necessary to maintain balance. In a study into the effects of vertebral fractures (not included in this thesis), a simple representation of the abdominal and dorsal muscles is included and the changing muscle force pattern is analysed due to the changing sagittal alignment of the spine (figure 10).

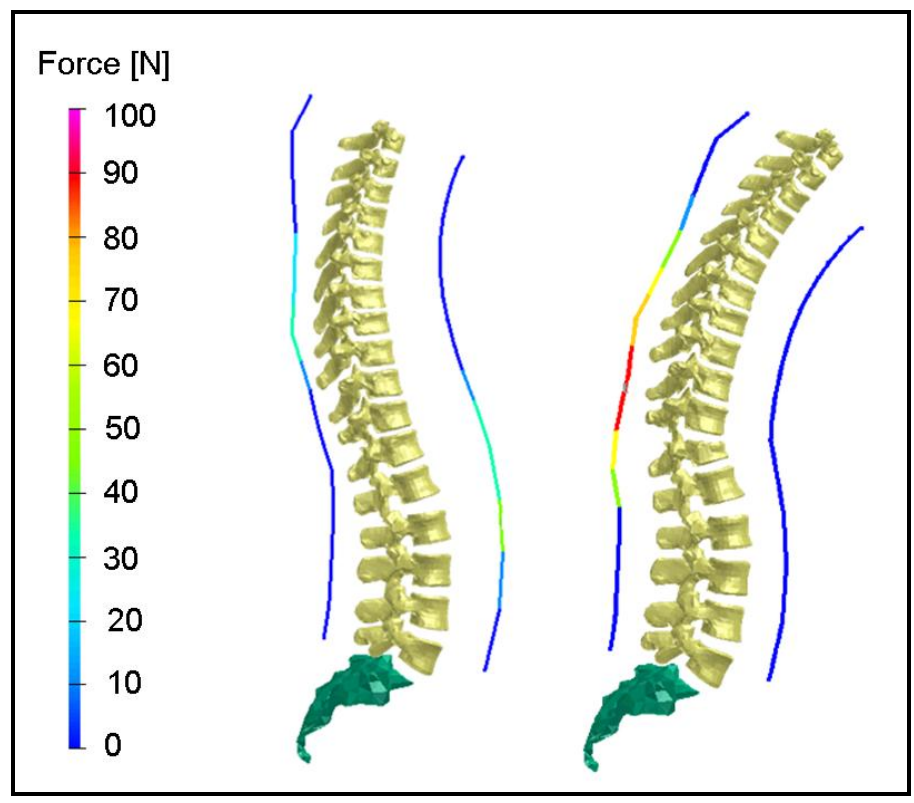

Figure 10: Influence of sagittal alignment on abdominal and dorsal muscle forces. Comparison of muscle forces in healthy situation (left) and spine with vertebral fracture at T12 (right), under gravitational loading. ${ }^{64}$

\subsubsection{Contact forces}

Forces can be determined at all contact surfaces. For example, the forces between the facet surfaces, but also between the rib-vertebral joints, can be analysed under various loading conditions. In this thesis, the facet contact forces under various 
loading conditions are used as a validation of the lumbar motion segment (see section 2.3.1.1).

In the current simulations, the implant is only modelled by its mechanical effects (forces and moments). In future research, when the actual implant is modelled, this tool can be used to analyse implant-spine interactions.

\subsubsection{Local stresses}

For all deformable elements the local stresses can be determined. This analysis is mainly used to determine the pressure profile in the intervertebral disc.

Comparison of the local disc stress can be a useful validation but it can also be used to analyse the effects of different loading conditions due to changing geometry of the spine in combination with gravity (wedge fracture, scoliosis) or the effects of implants on the pressure in adjacent discs (not presented in this thesis) can be analysed, as presented in figure 11.

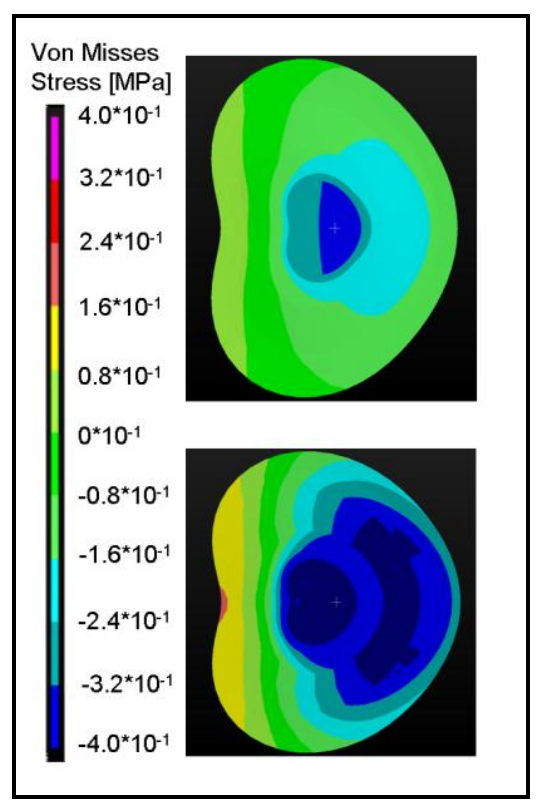

Figure 11: Influence of fusion implant on local stresses in adjacent disc. The Von Misses stress in the normal (upper) and implanted (lower) situation are compared ${ }^{65}$

\subsection{Validity of the models}

As already mentioned in the general introduction, verification of the modelling method and validation of the results is necessary to use the results with confidence. The modelling method is verified, using both tools implemented in the software and some manual checks. 
For the validation of the results, the previous described models are validated against experiments, using the methods from section 2.2. Due to limited space, not all validation aspects are elaborately presented in the articles (which form the following chapters); an overview of the validation process of the various models is therefore given in this section. Also, sensitivity analyses are conducted to analyse the effects of variation in the input parameters. A conclusion on the usability and validity of the model will end this chapter.

\subsubsection{Verification of the modelling method}

For all simulations, the consistency between the problem formulation and the results are checked, by checking symmetry (where applicable), gradients in the solution and the order of magnitude of the solution. III-conditioning and singularities of the model and quality and distortion of the elements were verified by the software.

The equilibrium of the model is manually checked by comparing the sum of the forces and moments at the supports with the externally applied loads. As further discussed in chapter 6 , this was a problem for the models of the thoracolumbar spine including the ribcage, but not for any of the other models.

The convergence of the used mesh size was verified for a motion segment model: it was verified that with a finer mesh the value of the stress in the elements did not change. Since the other models only need a more global solution, the same mesh is sufficient, or even too fine. To decrease computation time, a coarser mesh might be applied in future models; this was not further optimized in this project since the computation time was acceptable.

To improve accuracy, as many hexahedral and quadrilateral elements as possible are used. Only when the geometry could not be created without them, a limited number of tetrahedral and triangular elements are used. This was necessary to create the geometry of the ribs, intercostal muscles, costal cartilage, the diaphragm and the abdominal wall.

\subsubsection{Strategy for the validation of the various models}

As previously discussed, in vitro experiments in adolescents are lacking, and therefore only adult data can be used for validation. Therefore first an adult model of the spine was developed, which was validated against the adult data. To limit the influence of degenerative effects, care was taken to select studies that did not use aged spines, if possible. The adolescent model of the spine was obtained by scaling of the adult model, and could not be validated.

The model of the total ribcage was that of a ten-year old, and scaling of this model was not as straightforward as scaling of the spine model. To be able to use the available adult data, the reported range of motion from in vitro experiments in adults was scaled to compensate for the increased flexibility in adolescents.

This scaling is based on the measured greater flexibility for the paediatric cervical spine $^{66}$ compared to adult cervical spines ${ }^{67}$, on a finite element study on paediatric cervical spines ${ }^{14}$, and on our own study analysing the effects of adolescent growth on 
the lumbar spine ${ }^{68}$. The large difference between our study and the previous mentioned studies can be explained by the age difference in the subjects, and/or the different regions of the spine that are studied (table 11).

\begin{tabular}{|c|c|c|c|}
\hline & $\begin{array}{l}\text { In vitro measurements } \\
\text { children }^{66} \text { and adults }\end{array}$ & $\begin{array}{l}\text { FE model } \\
\text { material changes and } \\
\text { local geometry scaling }\end{array}$ & $\begin{array}{l}\text { FE model }{ }^{68} \\
\text { local geometry scaling }\end{array}$ \\
\hline Age children & $2-12$ years & 6 year & 10 year \\
\hline Spine region & $\mathrm{C} 2-\mathrm{T} 2$ & C4-C6 & L3-L4 \\
\hline Loading & $2.4 \mathrm{Nm}$ & $0.5 \mathrm{Nm}$ & $0-20 \mathrm{Nm}$ \\
\hline \multirow{4}{*}{$\begin{array}{r}\text { Increased } \\
\text { flexibility for } \\
\text { children }\end{array}$} & Flexion: $40 \%$ & Flexion: $150 \%$ & Flexion: $44 \%$ \\
\hline & Extension: $110 \%$ & Extension: $375 \%$ & Extension: $36 \%$ \\
\hline & & & Lateral bending: $36 \%$ \\
\hline & & & Axial rotation: $38 \%$ \\
\hline
\end{tabular}

Table 11: Reported increased flexibility for children compared to adults

For example, the larger reported effect in extension for both other studies can be explained by the changing orientation of the cervical facet joints before the age of 6 years. The orientation of the facet joints does not change during adolescent growth, resulting in smaller effects for the increased flexibility in extension for our study.

Since our study was the only study describing all motion directions and the only study analysing adolescent rather than paediatric spines, the scaling was based on our study. Since no large differences between the different motion directions are found, a single scaling factor (1.4) for the reported greater flexibility of adolescents compared to adults is assumed. It is pointed out that this data only considers a motion segment and geometrical changes during adolescent growth. More data on the changes in material properties, and/or other anatomical aspects of the spine and trunk are lacking at the moment, and are thus very useful for more detailed analyses and validation.

Since the focus in this thesis is mainly on the rotational movements (rotational degrees of freedom cause larger displacement than the translational ones and the rotational movements are more relevant in scoliosis), the validation of the various models also focuses on the rotational movements. Furthermore, only the main motions are validated, although coupled motions are present in both experimental data and simulations. The difficulty is the inconsistency in both the direction and amount of reported coupled motions in experiments, as a result of interpersonal differences in specimen and differences in testing methods ${ }^{69}$. Since the exact effects of the specimen and testing method cannot be isolated, the validation of coupled motions is both unworkable and unreliable.

In the in vitro experiments, the flexion/extension movement is often combined, due to difficulties in defining the "neutral position". This makes repetition of the experiments not straightforward, since the exact loading conditions are unknown. When only the 
range of applied flexion-extension moment is mentioned, it is assumed that flexion and extension contribute equally to this reported range.

Most data in literature concerns the lumbar and cervical spine, since low back pain and whiplash are common spine problems, concerning high costs for being on sickness benefit. Therefore, validation of the lumbar motion segment (L3-L4) was very extensively.

Since little data on thoracic motion segments was available, the validation of the thoracic spine focuses on multi-segments.

Since the costovertebral ligaments are anatomically intertwined with the spinal ligaments and the intervertebral disc, their removal is time-consuming and potentially harmful to the quality and biomechanical behaviour of the specimen ${ }^{70}$. For this reason, in vitro tests leave the posterior part of the ribs, intercostal muscles, costovertebral joints and ligaments attached. In chapter 5 , we show that this affects the biomechanics of the spine, especially in lateral bending. Therefore, all models of thoracic spine segments that are used in validation studies include the posterior part of the ribs, like in the experiments.

It is well-known that biomechanical modelling deals with a high variance and inaccuracy of the input data for the models. Therefore, a sensitivity study is often used to analyse the effects of this variance and/or inaccuracy of the input data on the outcome of the models.

In our models, a distinction can be made for the effects of the geometrical and material properties. In chapter 4, the effects of the interpersonal variance in spine geometry is analysed, resulting in a conclusion on the patient-specific geometrical data that is necessary to accurately predict patient-specific stiffness. Unfortunately, a comparable study on the effects of patient-specific material properties is not possible, since the inter-personal variance in material properties is not reported in literature.

\subsubsection{Validation of the spine models}

The load-displacement behaviour of the L3-L4 motion segment is validated for the four rotational movements (flexion, extension, lateral bending and axial rotation). Besides the behaviour of the intact motion segment, also the body-disc-body behaviour (motion segment without ligaments and facet joints) is validated. Also the simulated results of axial preloading combined with the rotational movements are compared to experimental data. The simulated results for all loading conditions lie well within the range of the experimental data, as shown in chapter 3 , figure 3 .

To validate the effect of the various ligaments and the facet joints, some of the ligaments and the facet joints of the L3-L4 segment are removed stepwise, as was also done in experiments by Heuer et al ${ }^{71}$. Comparative effects for the removal of ligaments and facet joints on the stiffness of the motion segment are shown for the simulations and experimental data in chapter 3 , figure 4 .

The effect of the facet joints in the L3-L4 are also validated by comparing the contact forces under various loading conditions with reported experimental data ${ }^{72-74}$ and simulations $^{75}$ (see figure 12). 


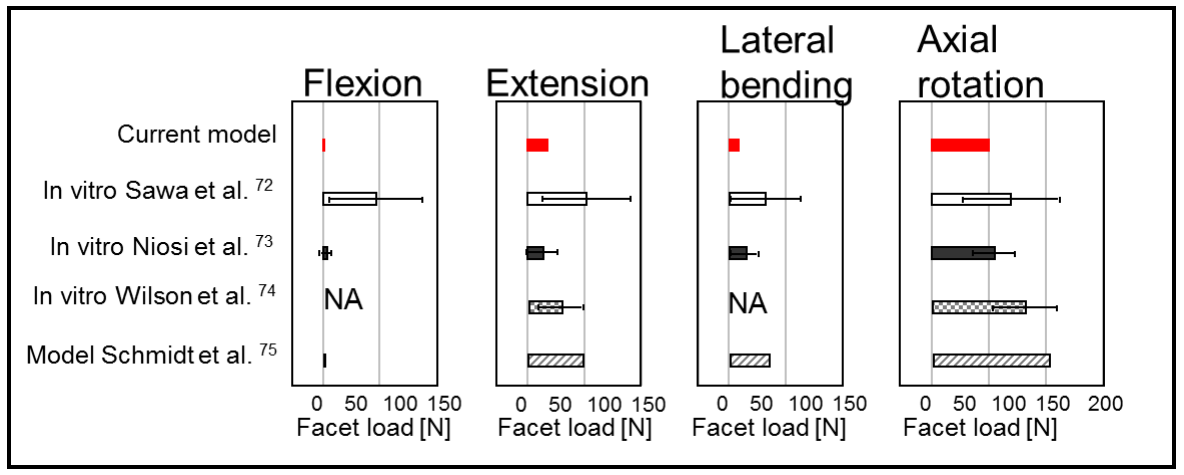

Figure 12: Facet loads when pure moments are applied on lumbar motion segments.

$N A=$ not available.

In all studies, a pure moment of $7.5 \mathrm{Nm}$ is applied and the corresponding facet load is measured. The current model shows similar trends as the reported data: the largest forces are found in axial rotation, followed by extension and lateral bending and negligible forces in flexion are found. The relative higher forces reported by Schmidt et $\mathrm{al}^{75}$ are related to the combined axial compression loading (500N), which increases the facet loading.

The total behaviour of the L3-L4 motion segment is not only validated by the magnitude of the rotation under various rotational loadings, but also by analysing the location of the momentary centre of rotation. A comparison between the simulated results and various experimental data is shown in figure 13.

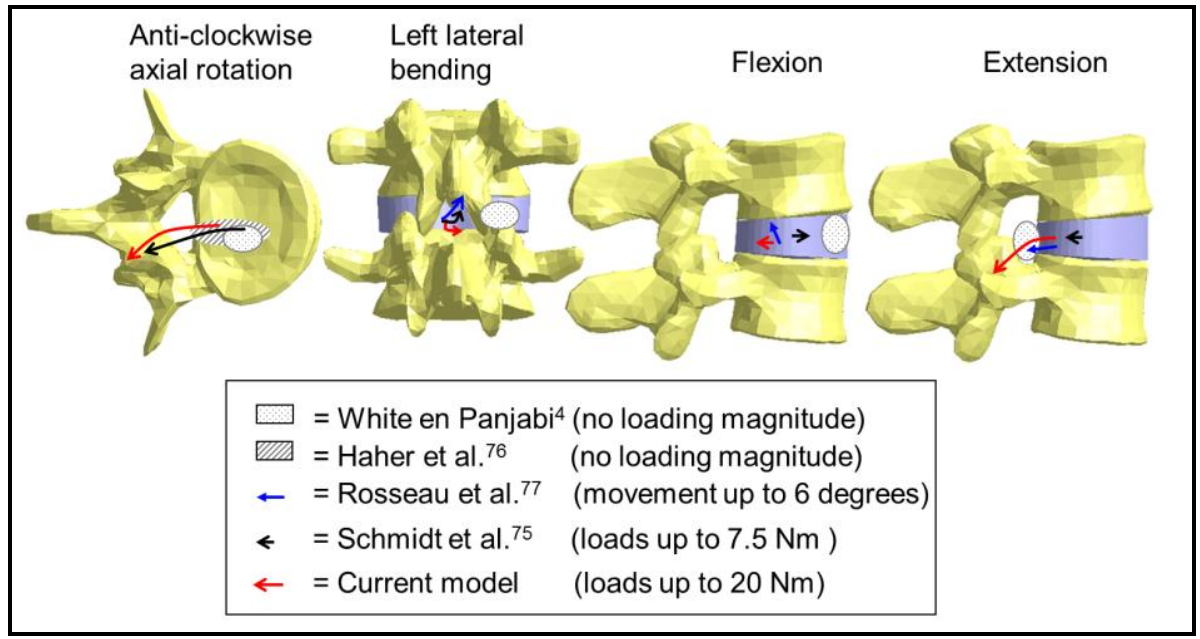

Figure 13: Movement of instantaneous centre of rotation during increasing moment for lumbar motion segments. 
In this figure the movement of the instantaneous centre of rotation with increasing moment is shown. This movement is due to the non-linear stiffness of the various parts; the position of this instantaneous centre of rotation corresponds with the position of the structure with the most dominating stiffness. The easiest example is axial rotation. With increasing clockwise moment and movement, the right facet joint becomes more dominant in limiting the motion, and the instantaneous centre of rotation moves from the vertebral body towards this facet joint, as shown by two studies $^{75,76}$ and found with the current simulations. White and Panjabi ${ }^{4}$ reported an instantaneous centre of rotation at the right posterior half of the disc, which is comparable to results of our simulations and those of Schmidt et $\mathrm{al}^{75}$ for small axial rotation moments. Since both White and Panjabi ${ }^{4}$ and Haher et $\mathrm{al}^{76}$ do not mention the magnitude of the loading, or the direction of movement of the centre of rotation with increasing load and exact validation of our simulations is not possible with their data. In left lateral bending, the disc, right ITL and right facet joint limit the movement, resulting in a movement of the instantaneous centre of rotation towards the right. Rousseau et $\mathrm{al}^{77}$ reported a move of the centre towards the left for both left and right lateral bending, which does not seem logical, and might be related to measurement errors or a preferred movement direction for the subjects $(n=12)$.

In flexion, the intervertebral disc and posterior ligaments are limiting the movement. In the simulations of the current model, the ligaments are more dominant than the disc, while in the reported literature the disc seems more limiting than the ligaments. This might be explained by the non-linear behaviour of the ligaments in combination with lower loading: the ligaments became the main limitation of the movement, when large moments are applied.

In extension the intervertebral disc, the ALL and the facet joints limit the movement. Our model and all reported literature show a movement of the instantaneous centre of rotation towards posterior, although the movement in our simulations is largest. This might be explained by a larger applied loading.

After the validation of a single motion segment, three stacked motion segments are validated against experiments conducted in this project ${ }^{78}$. The range of motion for the loading range $(-4 \mathrm{Nm}$ to $+4 \mathrm{Nm})$ are validated for four regions (T1-T4, T5-T8, T9T12, L1-L4) and 3 movement directions (flexion/extension, lateral bending and axial rotation) and presented in figure 14.

Most simulated results fall within the reported 1SD from the mean experimental value. The simulated results for flexion/extension of T5-T8 and lateral bending of T5T8 and T9-T12 are relative flexible, but still within the range of the experimental data. Comparable trends for the various regions and the movement directions are seen for the simulations and the experimental results. For both the simulations and the experimental data, the largest movement is seen in the high thoracic region (T1-T4) and the least movement is in the lumbar region (L1-L4) and/or low-thoracic (T9-T12) region

Finally, the mechanical behaviour of the total thoracic (T1-T12) adolescent spine is validated against in vitro experiments. To compensate for the larger reported motion in adolescents, the adult in vitro experimental results are scaled by a factor 1.4. This comparison is presented in chapter 6 , figure 3 . Both the experimental data and simulations show the largest motion in axial rotation (also the largest load!), followed 
ROM for load ranging from $-4 \mathrm{Nm}$ to $+4 \mathrm{Nm}$

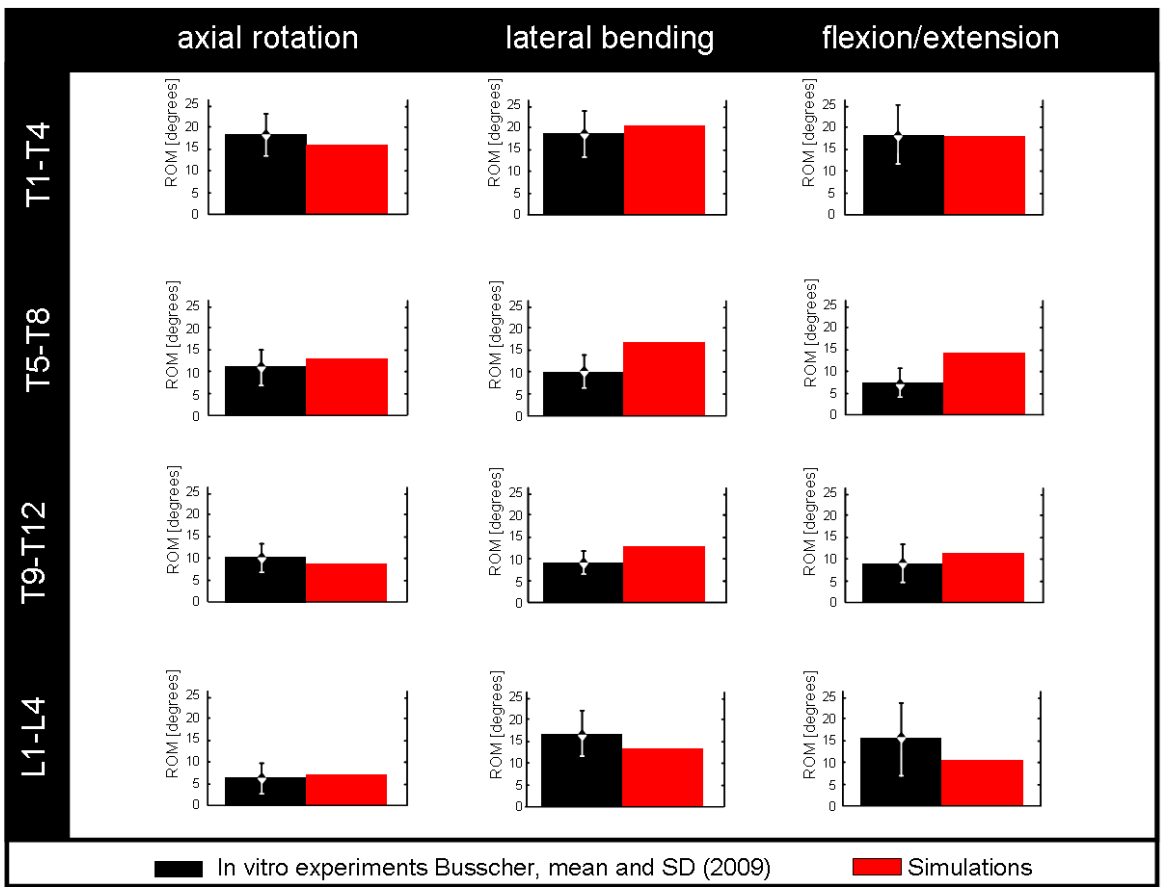

Figure 14: Validation of kinematics of multi-segment for four regions of the spine and three movement directions.

by flexion/extension and the least motion in lateral bending. All simulated results lie well within the reported experimental range.

\subsubsection{Validation of the ribcage models}

First of all, the mechanical behaviour of the rib-vertebral connections (joints and ligaments between the two adjacent vertebral bodies and the rib head, and the transverse process of the lower vertebra and the rib neck) are validated for adult motion segments including $10 \mathrm{~cm}$ ribs (T1-T2-R2, T5-T6-R6 and T9-T10-R10). This validation is presented in chapter 5 , figure 2 . The results show that the simulations compare well with the available data, for both the range of motion and the actual load-displacement curve of the rib joints. Both the experimental data and the simulations show the largest range of motion in torsion, although the smallest loads are applied. In the experimental data, however, no distinction between the various regions is made, so the regional effects cannot be verified.

The mechanical behaviour of the T1-T12 spine including the ribcage is compared to scaled in vitro experiments on adults, and is presented in chapter 6, figure 3. Again, the experimental data and simulations show comparable trends and similar 
mechanical effects for removal of the ribcage are seen. All simulated results lie well within the reported experimental range.

\subsubsection{Validation of the trunk models}

The mechanical behaviour of the T1-L5 spine including the ribcage and IAP is compared to in vivo experiments in adolescents ( 5 females, age 12-14 years) ${ }^{79}$. The presented experimental data are an average bending stiffness (EI) for the region between the hips and the shoulders, which is considered comparable to the T1-L5 region of the spine. The experimental loading is an anterior-posterior force at the sternum, with support forces at the hips and shoulders, resulting in a three point bending test in flexion, with a maximum moment of $2 \mathrm{Nm}$ at the sternum, and zero moment at the hips and shoulder. Furthermore, the moment of the muscles around the hip are neglected, resulting in an overestimation of the applied moment and the average bending stiffness. These effects, however, are considered small compared to the increased stiffness due to the presence of muscles, skin and arms in the experiments.

Therefore a comparison is also made to in vitro experiment determining the bending stiffness of the trunk ${ }^{80}$. Unfortunately, this concerns experiments on adult trunks, and again a scaling factor of 1.4 is used to compensate for the reported larger flexibility for adolescents (see table 11). The larger bending stiffness found in the in vitro experiment (even after scaling), can be explained by the larger applied moments (up to $48 \mathrm{Nm}$, instead of the simulated $2 \mathrm{Nm}$ ) and/or differences between the adult and adolescent trunk that are not captured by the estimated scaling factor.

An overview of the main differences between the experiments and the simulation, and the reported bending stiffness in flexion is presented in table 12.

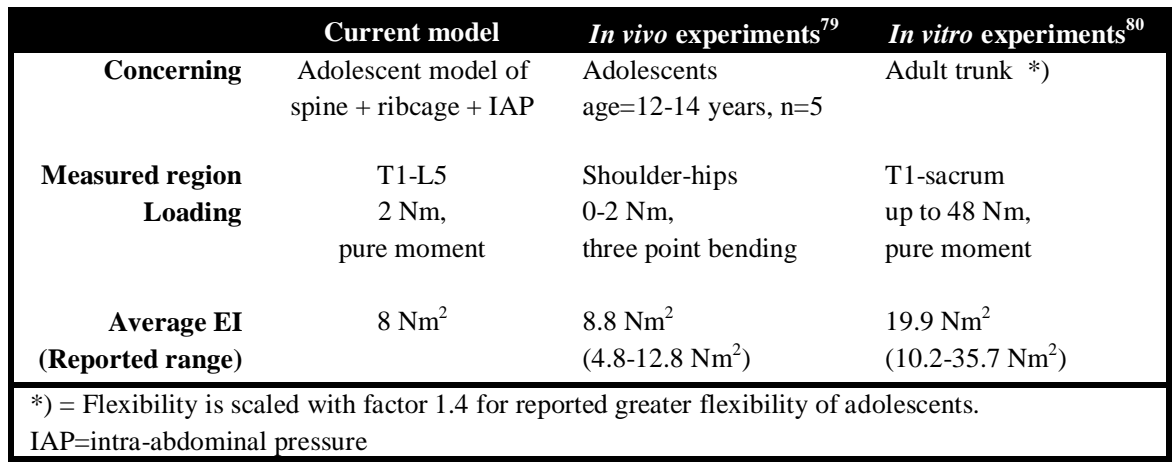

Table 12: Validation of the average flexion bending stiffness of the trunk model.

From this validation it can be concluded that the model is in good agreement with the reported in vivo data for the average flexion stiffness. The comparison with the in vitro experiments was less good, mainly due to the larger applied moments. This data was nevertheless selected, since this was the only literature comparing the stiffness of the total trunk for the various movement directions. These are presented in table 13. 


\begin{tabular}{|rcc|}
\hline & Current model & In vitro experiments ${ }^{\mathbf{8 0}} *$ ) \\
\hline $\begin{array}{r}\text { Average EI Axial rotation } \\
\text { (Reported range) }\end{array}$ & $7.3 \mathrm{Nm}^{2}$ & $42.0 \mathrm{Nm}^{2}$ \\
Average EI Lateral bending & & $\left(29-143 \mathrm{Nm}^{2}\right)$ \\
(Reported range) & $9.9 \mathrm{Nm}^{2}$ & 18.6 \\
Average EI Flexion & & $\left(14-71 \mathrm{Nm}^{2}\right)$ \\
(Reported range) & $8 \mathrm{Nm}^{2}$ & $19.9 \mathrm{Nm}^{2}$ \\
$\left(10-36 \mathrm{Nm}^{2}\right)$
\end{tabular}

Table 13: Validation of the average bending stiffness of the trunk model for various directions of movement.

The results in flexion and lateral bending are in good agreement, taking into account the larger applied loading in the in vitro experiment and assuming similar effects for both movement directions. The simulated average stiffness in axial rotation is much lower than the reported data, even when taking into account the higher loading in the experiments.

It is unclear whether the differences between the experimental data and the simulations are due to the difference between adults and adolescents (other than the pure scaling that is applied), differences in loading conditions (larger moments are applied in the experiments), incorrect modelling assumptions or a combination of all these factors.

\subsubsection{Validation of the scoliotic trunk model}

Validation of the scoliotic model is unfortunately not possible, due to lack of data. Controlled in vivo measurements on patients might provide more data, but then only validation of patient-specific models are possible, due to the large variance in anatomy and deformities of the trunk.

\subsection{Discussion}

One of the major simplifications of the motion segment model is the representation of the facet joints as parallel, planar surfaces, instead of a geometry that is curved in $3 \mathrm{D}$, as it is in reality.

Simulations into the effects of the geometry of the facet joints of the L3-L4 motion segment showed that especially the distance between the two facet surfaces has a large influence on the load-displacement behaviour of the motion segment. The simulations with the facet joints as a 3D curved surface did show an earlier contact between the facet surfaces, but due to the non-linear contact-definition, these effects are small (figure 15).

These simulations were only conducted for axial rotation loading, since the facet joints of the L3-L4 motion segment mainly influence the axial rotation behaviour. The effects for other movement directions and/or thoracic motion segments are expected to be of similar magnitude or smaller. 


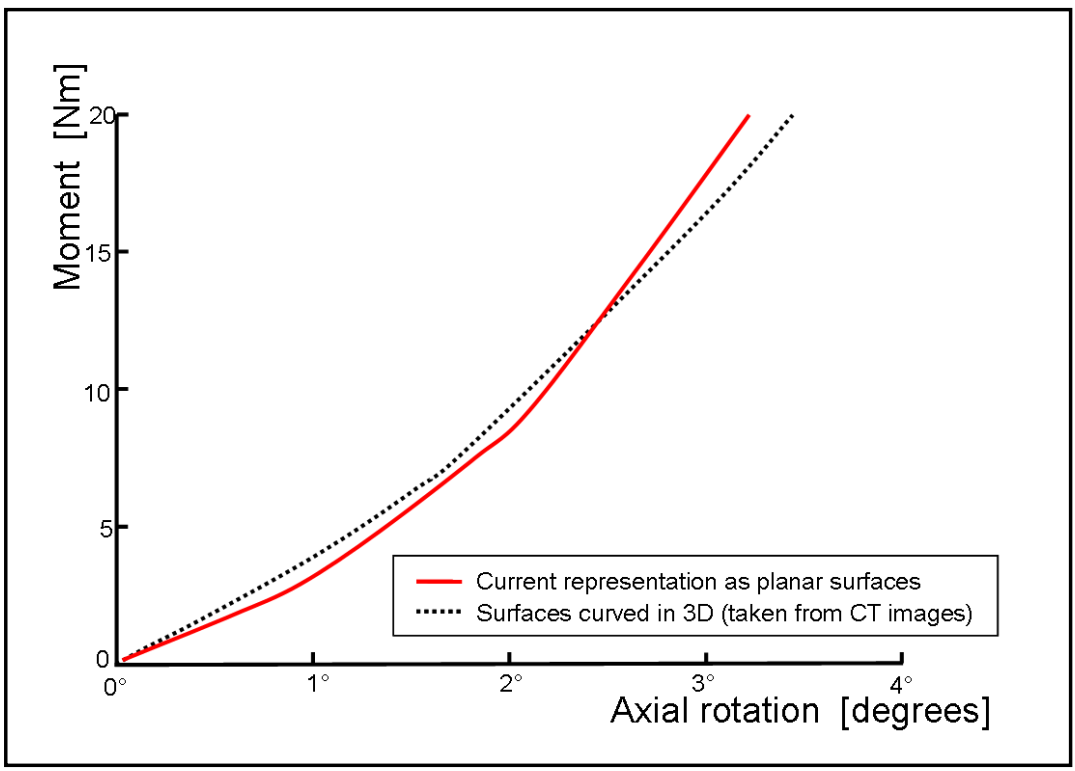

Figure 15: Influence of facet representation on the load-behaviour of the L3-L4 motion segment in axial rotation.

A similar simplification has been made for the contacts between the ribs and the vertebrae. Due to a different type of contact (penalty contact rather than describing force-penetration curve), a combination with a plane curved in 3D was not possible. It is expected that the effects of non-linearity in the contact definitions are more important than the 3D curvature of the planes. Since the mechanical behaviour of the isolated rib joints are validated, this assumption seems acceptable.

The elastic material properties of the soft tissues are also a simplification, because the actual properties, including non-linear and non-homogenous behaviour, are often unknown. Most important and limiting is the large variety between subjects, which makes an estimation of the average material properties difficult. One of the main assumptions for the material properties is the linear behaviour of the annulus fibres and neglecting the variation of fibre volume fraction and stiffness for different locations in the disc. Smit ${ }^{81}$ pointed out that non-linear stress-strain relationship for the annulus fibres is unnecessary when analysing physiological conditions, since the fibres are only strained in the linear range under physiological loading conditions and kinematic behaviour of the disc with linear fibres is realistic for these physiological loading cases. Neglecting the variation of fibre volume fraction and stiffness for different locations in the disc mainly influences the stress profile, but the influence on the load-displacement relations is negligible ${ }^{82}$.

The validity of the stress profiles of the disc is limited, due to a number of reasons. First of all, the vertebrae are modelled as rigid bodies, neglecting the deformity of the cartilage endplates, which can be considerable in compression ${ }^{83}$ and under larger bending moments ${ }^{84}$. 
Secondly, the variation of fibre volume fraction and stiffness for different locations in the disc (as described by Shirazi-Adl ${ }^{82,85}$ and Lu et al. ${ }^{86}$ ) is not modelled. In the outer layers of the annulus, the fibre volume fraction is higher and the elasticity modulus is higher than in the inner layers, while the current model represents an equivalent, but homogeneous distribution. This is expected to have an essential influence on the stress pattern within the intervertebral disc. When a homogeneous distribution of the stiffness and fibre volume fraction is assumed, the straining of the inner fibres due to bulging of the nucleus under axial compression will be more than the straining of the outer ones. So the homogeneous modelling will result in an overestimation of the stress in the centre of the disc and an underestimation of the stress in the outer part of the disc ${ }^{82}$.

Thirdly, the pretension in the annulus fibres is neglected, resulting in an underestimation of the hydrostatic pressure in the disc, although a similar effect (but smaller) is achieved by applying pretension in the spinal ligaments.

The stress profiles of the disc can therefore not be used in quantitative analyses, but only in qualitative analyses. In this thesis, the stress profiles of the disc are therefore not used.

The validation of the adolescent model is limited, due to lack of adolescent in vitro data. For the same reason, material properties of adolescents are also unknown, and available (young) adult data had to be used. Simulations with the adolescent model are not inconsistent with available literature, but true validation is not possible. This limitation also stresses the importance of a numerical model representative for the adolescent spine and trunk: in vitro testing is almost impossible in young humans. Often young animal models are used, but in vivo testing in animals (for instance pigs), has the disadvantage of different mechanical behaviour of the spine when compared to humans ${ }^{87}$. For scoliosis research, it is also important to realize that animals do not develop a natural scoliosis.

The current scoliotic model represents an average scoliosis and does not represent any specific patient. The chosen case represents the geometry of a patient that would be suitable for implantation of the new system, but since the true cause of idiopathic scoliosis is unknown, the current way of modelling scoliosis may not represent the true underlying effects of scoliosis.

The assumption of the stress release after the modelling of the scoliotic form, as mentioned before, can be seen as a combination of the adaptation and visco-elastic relaxation of the soft tissues in the human body. Deformities of the vertebral shape and the ribs, are adaptations of the bone to the altered loading conditions ${ }^{88}$. Since the remodelling of bone is taking more time than the remodelling of the soft tissues and the early progression of scoliosis is mainly due to intervertebral disc wedging ${ }^{89}$, the assumption of only modelling soft tissue adaptation and no bone remodelling, seems reasonable. The assumption of a total stress relaxation can be seen as a worst case scenario; the scoliotic spine has been truly adapted to the scoliosis and the scoliotic position is the new neutral position. 


\subsection{Conclusion on usability and validity of the model}

Biomechanical behaviour of the developed model of the adolescent spine, ribcage and trunk are compared to literature and show similar behaviour, although validation of the adolescent model and scoliotic models are difficult due to lack of data. No studies into material properties or (controlled) mechanical experiments have been published for the adolescent or scoliotic spine.

Furthermore, since the true cause of idiopathic scoliosis, is unknown, it is hard to model the underlying (mechanical) effects of scoliosis and changes (other than geometry) compared to the healthy spine.

However, results from the current model are not inconsistent with experimental data and knowledge about the biomechanics of the spine and trunk, the differences in the biomechanics of the spine between adults and adolescents, and the knowledge about the mechanics of the scoliotic spine.

It is therefore believed that the model is representative for the general, passive behaviour of the adolescent spine and trunk and can be used to predict scoliosis correction and answer biomechanical questions related to scoliosis. 


\section{References}

[1] M. Sharma, N.A. Langrana, J. Rodriquez, 'Role of ligaments and facets in lumbar spinal stability.'. Spine Vol. 20, pp. 887-900, 1995.

[2] J. Mizrahi, M.J. Silva, T.M. Keaveny, W.T. Edwards, W.C. Hayes, 'Finite-element stress analysis of the normal and osteoporotic lumbar vertebral body'. Spine Vol. 18, pp. 2088-2096, 1993.

[3] N.A. Langrana, S.P. Kale, W.T. Edwards, C.K. Lee, K.J. Kopacz, 'Measurement and analyses of the effects of adjacent end plate curvatures on vertebral stresses'. The Spine Journal Vol. 6, pp. 267-278., 2006.

[4] A.A. White, M.M. Panjabi (1978) Clinical Biomechanics of the Spine. 1st edn. Lippincott Philadelphia, USA

[5] F.J. Prestar, H. Frick, R. Putz, 'Ligamentous connections of the spinal process'. Anatomischer Anzeiger Vol. 159, pp. 259-268., 1985.

[6] D.J.A. Heylings, 'Supraspinous and interspinous ligaments of the human lumbar spine'. Journal of Anatomy Vol. 125, pp. 127-131., 1978.

[7] J. Chazal, A. Tanguy, M. Bourges, G. Gaurel, G. Escande, M. Guillot, G. Vanneuville, 'Biomechanical properties of spinal ligaments and a histological study of the supraspinal ligament in traction'. J Biomech Vol. 18, pp. 167-176., 1985.

[8] V.K. Goel, B.T. Monroe, L.G. Gilbertson, P. Brinckman, 'Interlaminar shear stresses and laminae separation in a disc. Finite element analysis of the L3-L4 motion segment subjected to axial compressive loads'. Spine Vol. 20, pp. 689-698., 1995.

[9] Y. Lu, W.C. Hutton, V.M. Gharpuray, 'Do bending, twisting and diurnal fluid changes in the disc affect the propensity to prolapse? A viscoelastic finite element model.'. Spine Vol. 21, pp. 2570-2579., 1996.

[10] J.O. Galante, 'Tensile properties of the human lumbar annulus fibrosus'. Acta Orthop Scand Vol. suppl, pp. 1-91, 1967.

[11] A.P. Dooris, V.K. Goel, N.M. Grosland, L.G. Gilbertson, D.G. Wilder, 'Load-sharing between anterior and posterior elements in a lumbar motion segment implanted with an artificial disc'. Spine Vol. 26, pp. E122-E129, 2001.

[12] F. Lavaste, W. Skalli, S. Robin, R. Roy-Camille, C. Mazel, 'Three-dimensional geometrical and mechanical modelling of the lumbar spine'. J Biomech Vol. 25, pp. 1153-1164., 1992.

[13] J.S. Little, P.S. Khalsa, 'Material properties of the human lumbar facet joint capsule'. $J$ Biomech Eng Vol. 127, pp. 15-24, 2005.

[14] S. Kumaresan, N. Yoganandan, F.A. Pintar, D.J. Maiman, S. Kuppa, 'Biomechanical study of pediatric human cervical spine: a finite element approach.'. J Biomech Eng Vol. 122, pp. 60$71,2000$.

[15] S.A. Voutsinas, G.D. MacEwan, 'Sagittal profiles of the spine.'. Clin Orthop Relat Res Vol. 210, pp. 235-242., 1986.

[16] J.-M. Mac-Thiong, E. Berthonnaud, J.R. Dimar, R. Betz, H. Labelle, 'Sagittal alignement of the spine and pelvis during growth.'. Spine Vol. 29, pp. 1642-1647., 2004.

[17] C. Giglio, J. Volpon, 'Development and evaluation of thoracic kyphosis and lumbar lordosis during growth'. J Child Orthop Vol. 1, pp. 187-193, 2007.

[18] A.G. Veldhuizen, P. Baas, P.J. Webb, 'Observations on the growth of the adolescent spine'. $J$ Bone Joint Surg Vol. 68-B, pp. 724-728, 1986.

[19] I.A.F. Stokes, L. Windisch, 'Vertebral height growth predominates over intervertebral disc height growth in adolescents with scoliosis.'. Spine Vol. 31, pp. 1600-1604., 2006.

[20] M.E. Brandner, 'Normal values of the vertebral body and intervertebral disk index during growth.'. AJR Vol. 110, pp. 618-627, 1970.

[21] A. Peacock, 'Observations on postnatal structure of intervertebral disc in man'. Journal of Anatomy Vol. 86 pp. 162-179., 1956.

[22] T.A.L. Wren, G.S. Beaupre, D.R. Carter, 'A model for loading-dependent growth, development, and adaptation of tendons and ligaments'. J Biomech Vol. 31, pp. 107-114., 1998. 
[23] R. Miyake, T. Ikata, S. Katoh, T. Morita, 'Morphologic analysis of the facet joint in the immature lumbosacral spine with special reference to spondylolysis'. Spine Vol. 21, pp. 783789., 1996.

[24] R.D. Labrom, 'Growth and maturation of the spine from birth to adolescence'. J Bone Joint Surg Vol. 89-A, pp. 3-7, 2007.

[25] S. Reichmann, 'The postnatal development of form and orientation of the lumbar intervertebral joint surfaces'. Anatomy and Embryology Vol. 133, pp. 102-123., 1971.

[26] J.E. Lonstein, J.M. Carlson, 'The prediction of curve progression in untreated idiopathic scoliosis during growth.'. J Bone Joint Surg Vol. 66-A, pp. 1061-1071., 1984.

[27] E. Rogala, D. Drummond, J. Gurr, 'Scoliosis: incidence and natural history. A prospective epidemiological study'. J Bone Joint Surg Vol. 60-A, pp. 173-176, 1978.

[28] A.J. Stirling, D. Howel, P.A. Millner, S. Sadiq, D. Sharples, R.A. Dickson, 'Late-onset idiopathic scoliosis in children six to fourteen years old. A cross-sectional prevalence study'. J Bone Joint Surg Vol. 78-A, pp. 1330-1336, 1996.

[29] S.L. Weinstein, L.A. Dolan, K.F. Spratt, K.K. Peterson, M.J. Spoonamore, I.V. Ponseti, 'Health and function of patients with untreated idiopathic scoliosis: A 50 -year natural history study'. JAMA Vol. 289, pp. 559-567, 2003.

[30] H.-K. Wong, J.H.P. Hui, U. Rajan, H.-P. Chia, 'Idiopathic scoliosis in singapore schoolchildren: A prevalence study 15 years into the screening program.'. Spine Vol. 30, pp. 1188-1196, 2005.

[31] J. Cruickshank, M. Koike, R. Dickson, 'Curve patterns in idiopathic scoliosis. A clinical and radiographic study'. J Bone Joint Surg Vol. 71-B, pp. 259-263, 1989.

[32] I. Villemure, C.-E. Aubin, G. Grimard, J. Dansereau, H. Labelle, 'Progression of vertebral and spinal three-dimensional deformitites in adolescent idiopathic scoliosis. A longitudinal study.'. Spine Vol. 26, pp. 2244-2250., 2001.

[33] Y.P. Charles, A. Diméglio, M. Marcoul, J.-F. Bourgin, A. Marcoul, M.-C. Bozonnat, 'Influence of idiopathic scoliosis on three-dimensional thoracic growth'. Spine Vol. 33, pp. 12091218., 2008.

[34] P. Openshaw, S. Edwards, P. Helms, 'Changes in rib cage geometry during childhood'. Thorax Vol. 39, pp. 624-627, 1984.

[35] S. Na, S. Sah, D. Hwang, H.Y. Choi, I. Lee, J. Lee, 'Numerical simulation of out-of-position driver side airbag deployment with small female model'. JSAE Annual Congress Vol. 8, pp. 5-8, 2003.

[36] I.A. Kapandji, L.H. Honoré (1985) The physiology of the joints. Volume 3: The trunk and the vertebral column. 1st edn. Ferdinand Enke Verlag, Stuttgart, Germany

[37] R. Putz, R. Pabst (eds) (1993) Sobotta atlas of human anatomy., vol 2: Trunk, Viscera, Lower Limb. 20th edn. Bohn Stafleu Van Loghum Houten, The Netherlands

[38] J.D. Stitzel The role of local material properties in modeling fracture tolerance of the human thorax. PhD thesis, Viriginia polytechnic institute and state university/ Blacksburg, Virginia, 2003.

[39] T.A. Wilson, A. Legrand, P.-A. Gevenois, A. De Troyer, 'Respiratory effects of the external and internal intercostal muscles in humans'. The Journal of Physiology Vol. 530, pp. 319-330, 2001.

[40] M.A. Nussbaum, B.J. Martin, D.B. Chaffin, 'A neural network model for simulation of torso muscle coordination'. J Biomech Vol. 30, pp. 251-258, 1997.

[41] H. Yamada (1970) Strength of biological materials. 1st edn. Williams and Wilkins, Baltimore (MD, USA)

[42] M.L. Sham, T. Zander, G. Rohlmann, G. Bergmann, 'Effects of the ribcage on thoracic spine flexibility'. Biomedizinische technik Vol. 50, pp. 361-365., 2005.

[43] R.F. Closkey, A.B. Schultz, C.W. Luchies, 'A model for studies of the deformable rib cage'. J Biomech Vol. 25, pp. 529-539, 1992.

[44] T. Andriacchi, A. Schultz, T. Belytschko, J. Galante, 'A model for studies of mechanical interactions between the human spine and rib cage'. J Biomech Vol. 7, pp. 497-507, 1974.

[45] Y.C. Fung (1993) Biomechanics : mechanical properties of living tissues. 2nd edn. Springer 
[46] G. Kraan, P. Hoogland, P. Wuisman, 'Extraforaminal ligament attachments of the thoracic spinal nerves in humans'. European Spine Journal Vol. 18, pp. 490-498, 2009.

[47] A.F. Ibrahim, H.H. Darwish, 'The costotransverse ligaments in human: A detailed anatomical study'. Clinical Anatomy Vol. 18, pp. 340-345, 2005.

[48] H. Jiang, J.V. Raso, M.J. Moreau, G. Russell, D.L. Hill, K.M. Bagnall, 'Quantitative morphology of the lateral ligaments of the spine: Assessment of their importance in maintaining lateral stability'. Spine Vol. 19, pp. 2676-2682, 1994.

[49] J. Cholewicki, K. Juluru, S.M. McGill, 'Intra-abdominal pressure mechanism for stabilizing the lumbar spine'. J Biomech Vol. 32, pp. 13-17, 1999.

[50] S.M. McGill, R.W. Norman, 'Reassessment of the role of intra-abdominal pressure in spinal compression '. Ergonomics Vol. 30, pp. 1565-1588., 1987.

[51] S.M. McGill, R.W. Norman (1993) Low back biomechanics in industry: the prevention of injury through safe lifting. In: Current issues in biomechanics. Human Kinetics Publishers, Champaign, IL., pp 69-120

[52] J. Cholewicki, K. Juluru, A. Radebold, M.M. Panjabi, S.M. McGill, 'Lumbar spine stability can be augmented with an abdominal belt and/or increased intra-abdominal pressure'. European Spine Journal Vol. 8, pp. 388-395, 1999.

[53] I.A.F. Stokes, M. Gardner-Morse, 'Quantitative anatomy of the lumbar musculature.'. J Biomech Vol. 32, pp. 311-316, 1999.

[54] N. Arjmand, A. Shirazi-Adl, 'Role of intra-abdominal pressure in the unloading and stabilization of the human spine during static lifting tasks'. European Spine Journal Vol. 15, pp. 1265-1275, 2006.

[55] M. Gatton, M. Pearcy, G. Pettet, 'Modelling the line of action for the oblique abdominal muscles using an elliptical torso model'. J Biomech Vol. 34, pp. 1203-1207, 2001.

[56] I.A.F. Stokes, M.G. Gardner-Morse, S.M. Henry, 'Intra-abdominal pressure and abdominal wall muscular function: Spinal unloading mechanism'. Clin Biomech Vol. 25, pp. 859-866, 2010.

[57] A. Schultz, G. Andersson, R. Ortengren, K. Haderspeck, A. Nachemson, 'Loads on the lumbar spine. Validation of a biomechanical analysis by measurements of intradiscal pressures and myoelectric signals'. J Bone Joint Surg Vol. 64-A, pp. 713-720, 1982.

[58] M. Essendorp Significance of intra-abdominal pressure in work related trunk-loading. PhD thesis, National Institute of Occupational Health/ Copenhagen, Denmark, 2003.

[59] A.L. Nachemson, G.B.J. Andersson, A.B. Schultz, 'Valsalva maneuver biomechanics: effects on lumbar trunk loads of elevated intraabdominal pressures'. Spine Vol. 11, pp. 476479., 1986.

[60] H. Shin, M.J. Kim, 'Subcutaneous tissue thickness in children with type 1 diabetes'. Journal of Advanced Nursing Vol. 54, pp. 29-34, 2006.

[61] M.A. Gibney, C.H. Arce, K.J. Byron, L.J. Hirsch, 'Skin and subcutaneous adipose layer thickness in adults with diabetes at sites used for insulin injections: implications for needle length recommendations'. Current Medical Research and Opinion Vol. 26, pp. 1519-1530, 2010. [62] A.G. Cresswell, H. Grundström, A. Thorstensson, 'Observations on intra-abdominal pressure and patterns of abdominal intra-muscular activity in man'. Acta Physiologica Scandinavia Vol. 144, pp. 409-418., 1992.

[63] A. Cresswell, A. Thorstensson, 'Changes in intra-abdominal pressure, trunk muscle activation and force during isokinetic lifting and lowering'. European Journal of Applied Physiology and Occupational Physiology Vol. 68, pp. 315-321, 1994.

[64] M.S. Sietsma, G.J.M. Meijer, N. Verdonschot, J. Homminga, A.G. Veldhuizen, 'Altered sagittal alignment, consequences on spinal loads and trunk muscle forces; a finite-element study.'. To be submitted.

[65] J.J. De Jong De invloed van de stijfheid van eht posterieure fixatie systeem op de mechanica van de wervelkolom. Bachelor Thesis, University of Twente/ 2009.

[66] J. Ouyang, Q. Zhu, W. Zhao, Y. Xu, W.J. Chen, S. Zhong, 'Biomechanical assessment of the pediatric cervical spine under bending and tensile loading'. Spine Vol. 30, pp. E716-E723, 2005.

[67] R.D. Rao, M. Wang, L.M. McGrady, T.J. Perlewitz, K.S. David, 'Does anterior plating of the cervical spine predispose to adjacent segment changes? '. Spine Vol. 30, pp. 2788-2792, 2005. 
[68] G.J.M. Meijer, J. Homminga, E.E.G. Hekman, A.G. Veldhuizen, G.J. Verkerke, 'The effect of three-dimensional geometrical changes during adolescent growth on the biomechanics of a spinal motion segment'. J Biomech Vol. 43, pp. 1590-1597, 2010.

[69] P.S. Sizer, J.-M. Brismée, C. Cook, 'Coupling behavior of the thoracic spine: a systematic review of the literature'. Journal of Manipulative and Physiological Therapeutics Vol. 30, pp. 390-399, 2007.

[70] H.J. Wilke, B. Jungkanz, K. Wenger, L.E. Claes, 'Spinal segment range of motion as a function of in vitro test conditions: effects of exposure period, accumulated cycles, angulardeformation rate and moisture condition.'. Anat Rec Vol. 251, pp. 15-19, 1998.

[71] F. Heuer, H. Schmidt, Z. Klezl, L. Claes, H.-J. Wilke, 'Stepwise reduction of functional spinal structures increase range of motion and change lordosis angle'. J Biomech Vol. 40, pp. 271-280., 2007.

[72] A.G.U. Sawa, N.R. Crawford, 'The use of surface strain data and a neural networks solution method to determine lumbar facet joint loads during in vitro spine testing'. J Biomech Vol. 41 , pp. 2647-2653., 2008.

[73] C.A. Niosi, D.C. Wilson, Q. Zhu, O. Keynan, D.R. Wilson, T.R. Oxland, 'The effect of dynamic posterior stabilization on facet joint contact forces; An in vitro investigation'. Spine Vol. 33, pp. 19-26., 2008.

[74] D.C. Wilson, C.A. Niosi, Q.A. Zhu, T.R. Oxland, D.R. Wilson, 'Accuracy and repeatability of a new method for measuring facet loads in the lumbar spine'. J Biomech Vol. 39, pp. 348-353., 2006.

[75] H. Schmidt, F. Heuer, L. Claes, H.-J. Wilke, 'The relation between the instantaneous center of rotation and facet joint forces - A finite element analysis'. Clin Biomech Vol. 23, pp. 270-278., 2008.

[76] T.R. Haher, M. O'Brien, W.T. Felmly, D. Welin, G. Perrier, J. Choueka, V. Devlin, A. Vassiliou, G. Chow, 'Instantaneous Axis of Rotation as a Function of the Three Columns of the Spine'. Spine Vol. 17, pp. S149-S154, 1992.

[77] M.A. Rousseau, D.S. Bradford, T. Hadi, K. Pedersen, J.C. Lotz, 'The instant axis of rotation influences facet forces at L5/S1 during flexion/extension and lateral bending'. European Spine Journal Vol. 15, pp. 299-307., 2006.

[78] I. Busscher, J.H. van Dieen, I. Kingma, A.J. van der Veen, G.J. Verkerke, A.G. Veldhuizen, 'Biomechanical characteristics of different regions of the human spine: an in vitro study on multilevel spinal segments'. Spine Vol. 34, pp. 2858-2864, 2009.

[79] P.J.M. Scholten, A.G. Veldhuizen, 'The bending stiffness of the trunk'. Spine Vol. 11, pp. 463-467., 1986.

[80] M.F. Eijkelkamp, E. Otten, A.G. Veldhuizen, J.R. van Horn, G.J. Verkerke (2002) Measurement of the compliance of the human torso. In: On the development of an artificial intervertebral disc. Rijksuniversiteit Groningen, PhD-thesis,

[81] T.H. Smit The mechnical significance of the trabecular bone architecture in a human vertebra. PhD Thesis, Technischen Universitaet Hanburg-Harburg/ 1996.

[82] A. Shirazi-Adl, 'On the fibre composite material models of disc annulus - comparison of predicted stresses.'. J Biomech Vol. 22, pp. 357-365, 1989.

[83] J.J. MacLean, J.P. Owen, J.C. Iatridis, 'Role of endplates in contributing to compression behaviors of motion segments and intervertebral discs'. J Biomech Vol. 40, pp. 55-63, 2007.

[84] A. Shirazi-Adl, 'Analysis of role of bone compliance on mechanics of a lumbar motion segment'. J Biomech Eng Vol. 116, pp. 408-412., 1994.

[85] A. Shirazi-Adl, S.C. Shrivastava, A.M. Ahmad, 'Stress analysis of the lumbar disc-body unit in compression. A three-dimensional nonlinear finite element study.'. Spine Vol. 9, pp. 120-134., 1984.

[86] Y.M. Lu, W.C. Hutton, V.M. Gharpuray, 'Can variations in intervertebral disc height affect the mechanical function of the disc?'. Spine Vol. 21, pp. 2208-2216, 1996.

[87] I. Busscher, A.J. van der Veen, J.H. van Dieen, I. Kingma, G.J. Verkerke, A.G. Veldhuizen, 'In vitro biomechanical characteristics of the spine: A comparison between human and porcine spinal segments'. Spine Vol. 35, pp. E35-E42, 2010. 
[88] D.J. Wever, A.G. Veldhuizen, J.P. Klein, P.J. Webb, G. Nijenbanning, J.C. Cool, J.R. van Horn, 'A biomechanical analysis of the vertebral and rib deformities in structural scoliosis'. European Spine Journal Vol. 8, pp. 252-260, 1999.

[89] R.E. Will, I.A. Stokes, X. Qiu, M.R. Walker, J.O. Sanders, 'Cobb angle progression in adolescent scoliosis begins at the intervertebral disc'. Spine Vol. 34, pp. 2782-2786, 2009. 



\section{Chapter 3}

The effect of three-dimensional geometrical changes during adolescent growth on the biomechanics of a spinal motion segment

G.J.M. Meijer, J. Homminga, E.E.G. Hekman, A.G. Veldhuizen, G.J. Verkerke

Journal of Biomechanics v. 43: pp. 1590-1597, 2010

Reprinted with permission from Elsevier 
The effects of three-dimensional geometrical changes during adolescent growth on the biomechanics of a spinal motion segment

\begin{abstract}
During adolescent growth, vertebrae and intervertebral discs undergo various geometrical changes. Although such changes are well known, their effect on spinal stiffness remains poorly understood. However, this understanding is essential in the treatment of spinal abnormalities during growth, such as scoliosis.

A finite element model of an L3-L4 motion segment was developed, validated and applied to study the quantitative effects of changing geometry during adolescent growth on spinal stiffness in flexion, extension, lateral bending and axial rotation. Height, width and depth of the vertebrae and intervertebral disc were varied, as were the width of the transverse processes, the length of the spinous process, the size of the nucleus, facet joint areas and ligament size. These variations were based on average growth data for girls, as reported in literature.

Overall, adolescent growth increases the stiffness with $36 \%$ (lateral bending and extension) to $44 \%$ (flexion). Two thirds of this increase occurs between 10 and 14 years of age and the last third between 14 years of age and maturity.

Although the height is the largest geometrical change during adolescent growth, its effect on the biomechanics is small. The depth increase of the disc and vertebrae significantly affects the stiffness in all directions, while the width increase mainly affects the lateral bending stiffness. Hence, when analysing the biomechanics of the growing adolescent spine (for instance in scoliosis research), the inclusion of depth and width changes, in addition to the usually implemented height change, is essential.
\end{abstract}




\subsection{Introduction}

Scoliosis, a three-dimensional deformity of the spinal column, has a prevalence of 2$4 \%$ in adolescents. In more severe cases bracing or surgical treatment is needed for correction of the spinal curvature ${ }^{1,2}$. The achievable degree of correction is highly dependent on the stiffness of the spine; a clinical assessment of spinal stiffness is thus an important part of the current treatment planning ${ }^{3-5}$. If treatment is applied during adolescence, the spinal stiffness will change as the patient grows and the expected degree of correction depends on this changing stiffness.

Two in vivo studies in healthy adolescents have found a decreasing range of motion with increasing age during adolescence ${ }^{6,7}$. Unfortunately, as neither study measured the muscle force or activity, no clear conclusion about the changes in stiffness of the spine during adolescence can be drawn.

Finite element studies use biomechanical models of the growing adolecent spine to study long-term effects of scoliosis treatment ${ }^{8}$, various pathogenesis hypotheses ${ }^{9}$ or progression hypotheses for adolescent scoliosis ${ }^{10}$. A limitation is that, while height growth of vertebral bodies and discs are implemented, changes of all other dimensions are either neglected or not based on actual growth data. Growth of facets, ligaments and processi of the vertebrae are not modelled, while their effect on the biomechanics may be substantial.

The only study that included effects of full three dimensional growth on the biomechanics was a modelling study on paediatric versus adult cervical spines. This study found that stiffness increases during paediatric growth and that overall geometrical scaling with one scaling factor cannot capture the true effects of growth on the biomechanics ${ }^{11}$.

The goal of our study is to determine the quantitative effects of three-dimensional geometrical adolescent growth on spinal stiffness. By using a validated finite element model (FE-model) of a spinal motion segment, we are able to also study the separate effects of the geometric parameters on spinal stiffness.

\subsection{Methods}

\subsubsection{Finite element model}

Scoliosis typically occurs in the thoracic and thoracolumbar region. Unfortunately, there is very little data on material properties and growth related changes of the thoracic spine. Moreover, detailed in vitro tests required for model validation are lacking altogether. We therefore chose to use a validated lumbar model and project those results on the thoracic spine rather than to use an invalidated thoracic model and incomplete growth data.

We thus developed a FE-model of an adult L3-L4 motion segment (figure 1). As the spine's flexibility is dominated by the disc, the vertebrae are modelled as rigid bodies, while disc and ligaments are represented by a deformable mesh. An overview of the element types and mechanical properties are given in table 1. 


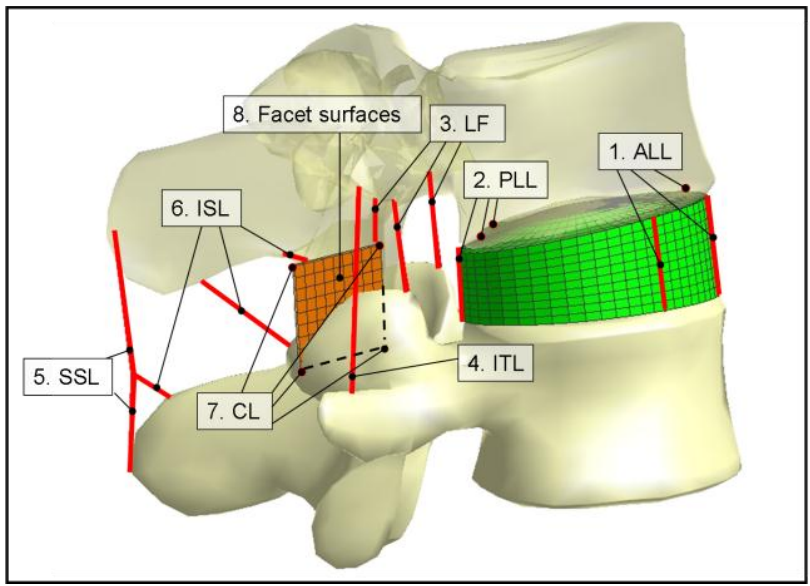

Figure 1: Finite element model of L3-L4 motion segment. For clarity only the right facet surfaces, CL and ITL are shown. Note that the vertebrae are rigid bodies. For explanation of the abbreviations of the ligaments, see text.

\begin{tabular}{|rlll|} 
Structure & Element type & $\begin{array}{l}\text { Mechanical } \\
\text { properties }\end{array}$ & Ref. \\
\hline Nucleus & 8 node linear & $\mathrm{E}=1 \mathrm{MPa}, \mathrm{v}=0.495$ & 12 \\
Annulus matrix & 8 node linear & $\mathrm{E}=2 \mathrm{MPa}, \mathrm{v}=0.45$ & 13 \\
Annulus fibres & Tension-only fibres in matrix & $\mathrm{E}=450 \mathrm{MPa}$ & 14 \\
Ligaments & Tension-only bars & See table 2 & See table 2 \\
Facet surfaces & 4 node shell & See table 3 & - \\
Vertebrae & Rigid bodies & - & - \\
\hline
\end{tabular}

Table 1: Element types and mechanical properties for the various structures in the L3-L4 model

The geometry of the disc and vertebrae was based on parameters from literature ${ }^{15-17}$, see figure 2.

The intervertebral disc consisted of an incompressible nucleus (occupying $43 \%$ of the midsagittal cross-sectional area of the disc) and an annulus which contains an isotropic matrix and circumferential tension-only fibres oriented at $+30^{\circ}$ and $-30^{\circ}$ to the transverse plane (volume ratio $=16 \%$ ).

All spinal ligaments - anterior longitudinal ligament (ALL), posterior longitudinal ligament (PLL), ligament flavum (LF), interspinous ligament (ISL), supraspinous ligament (SSL), intertransverse ligaments (ITL) and capsular ligaments (CL) - were included. The ISL was modelled with three elements to capture its different directions: the middle element representing half of the total area and the other two each representing $25 \%{ }^{18}$. The ALL, PLL, LF and CL were modelled with multiple elements, of equal area, to capture their width.

The length of each ligament is determined by the geometry of the model: it may thus differ from literature values. To avoid potential effects on motion segment behaviour, 
The effects of three-dimensional geometrical changes during adolescent growth on the biomechanics of a spinal motion segment

the stiffness, rather than Young's modulus, of the experimental literature data was used. The Young's modulus for the modelled ligament ( $\left.E_{\text {model }}\right)$ followed from this

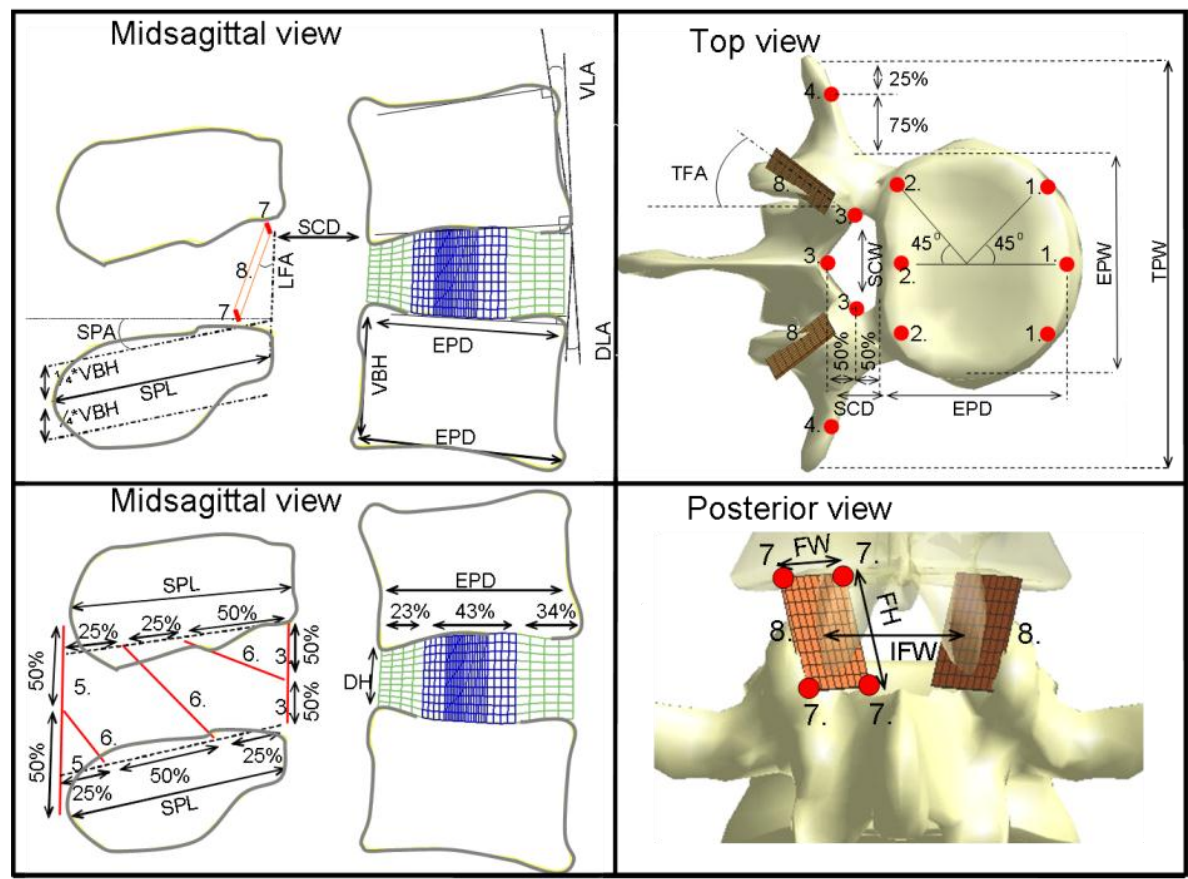

Figure 2: Parameters used to define the geometry of the motion segment. The numbering refers to structures presented in figure 1. For explanation of the abbreviations, see table 4.

experimental stiffness ( $\left.k_{\text {literature }}\right)$, the modelled length $\left(L_{\text {model }}\right)$ and published crosssectional area ( $\left.A_{\text {literature }}\right)$ using the following equation:

$\mathrm{E}_{\text {model }}=\frac{\mathrm{k}_{\text {literature }} * \mathrm{~L}_{\text {model }}}{\mathrm{A}_{\text {literature }}}$

(equation 1)

The non-linear behaviour of the ligaments was implemented by using three linear elastic strain regions, see table 2 . The prestrain of each ligament was set to $10 \%$ of the maximum strain of the first strain region, which compared well with forces reported in previous studies ${ }^{19-21}$. 
The effects of three-dimensional geometrical changes during adolescent growth on the biomechanics of a spinal motion segment

\begin{tabular}{|c|c|c|c|c|c|c|}
\hline Ligament & $\begin{array}{l}\text { Stiffness } \\
{[\mathbf{N} / \mathbf{m m}]} \\
k_{\text {literature }} \\
1^{\text {st }} \text { region }\end{array}$ & $\begin{array}{l}\text { Stiffness } \\
{[\mathbf{N} / \mathbf{m m}]} \\
k_{\text {literature }} \\
2^{\text {nd }} \text { region }\end{array}$ & $\begin{array}{l}\text { Stiffness } \\
{[\mathbf{N} / \mathbf{m m}]} \\
k_{\text {literature }} \\
3^{\text {rd }} \text { region }\end{array}$ & $\begin{array}{l}\text { Area } \\
{\left[\mathbf{m m}^{2}\right]} \\
A_{\text {literature }}\end{array}$ & $\begin{array}{l}\text { Length } \\
\text { [mm] } \\
L_{\text {model }}\end{array}$ & $\begin{array}{l}\text { Pretension } \\
{[\mathbf{N}]}\end{array}$ \\
\hline $\mathbf{A L L}$ & $52(0-12 \%)^{22}$ & $129(12-45 \%)^{22}$ & $65(45-58 \%)^{22}$ & $66^{22}$ & 12.5 & 7.7 \\
\hline PLL & $50(0-9 \%)^{22}$ & $118(9-34 \%)^{22}$ & $38(34-45 \%)^{22}$ & $26^{22}$ & 9.0 & 4.1 \\
\hline $\mathbf{L F}$ & $65(0-5 \%)^{14}$ & $85(5-50 \%)^{14}$ & $26(50-58 \%)^{b}$ & $39^{22}$ & 9.0 & 2.9 \\
\hline ISL & $24(0-12 \%)^{22}$ & $53(12-30 \%)^{22}$ & $25(30-40 \%)^{22}$ & $40^{14}$ & 13.8 & $3.9^{c}$ \\
\hline SSL & $18(0-12 \%)^{22}$ & $40(12-30 \%)^{22}$ & $19(30-40 \%)^{22}$ & $30^{14}$ & 24.8 & 5.3 \\
\hline ITL $^{\mathrm{a}}$ & $28(0-9 \%)^{22}$ & $137(9-15 \%)^{22}$ & $46(15-17 \%)^{22}$ & $2^{22}$ & 11.9 & 0.2 \\
\hline $\mathbf{C L}^{\mathrm{a}}$ & $18(0-100 \%)^{23}$ & $45(100-200 \%)^{b}$ & $15(200-300 \%)^{b}$ & $30^{14}$ & 0.6 & 1.0 \\
\hline $\begin{array}{l}\text { a) pers } \\
\text { b) extra } \\
\text { c) only }\end{array}$ & ed & 1 & & & & \\
\hline
\end{tabular}

Table 2: Ligament properties

The facet articular surfaces were modelled as parallel planes with a gap of $0.6 \mathrm{~mm}$ and a friction coefficient of 0.01 . Transverse and longitudinal facet angles were based on literature ${ }^{17}$. The non-linear stiffness of cartilage is implemented into the contact definition of the surfaces (table 3).

\begin{tabular}{|cc|}
\hline $\begin{array}{c}\text { Compression } \\
{[\mathbf{m m}]}\end{array}$ & $\begin{array}{c}\text { Stiffness } \\
{[\mathbf{N} / \mathbf{m}]}\end{array}$ \\
\hline $0.0-0.25$ & 0.1 \\
$0.25-0.5$ & 0.5 \\
$0.5-1.0$ & 4.2 \\
$1.0-1.5$ & 7.5 \\
$1.5-2.0$ & 22.5 \\
$>2.0$ & 90.0 \\
\hline
\end{tabular}

Table 3: Non-linear material properties representing facet articular cartilage

For all FE simulations PAM-CRASH software was used (ESI-group, France).

\subsubsection{Model validation against in vitro tests}

The complete adult motion segment was validated against several published in vitro experiments on lumbar motion segments (20-60 years) ${ }^{24-27}$. The relative contribution of the ligaments, facets and disc were validated by comparing experimental results on various dissection stages with simulations of similar stages. In the last stage all posterior elements and ligaments were removed, leaving only the vertebral bodies and the disc (body-disc-body). To make the comparison of the relative contribution easier, the movement for both the simulation and the experiment are set to $0 \%$ for the intact motion segment and to $100 \%$ for the body-disc-body. The two stages lying in between are thus normalised to the difference between the intact and body-discbody results. The body-disc-body was also validated.

Load cases in the simulations were similar to the experiments in literature: the lower vertebra was fixed and pure moments were applied to the local axis system in the 
middle of the upper vertebral body. We analysed loads from 0 to $10 \mathrm{Nm}$, which is a little more than what is advised for testing lumbar implants ${ }^{28}$. The rotation of the local axis system was used to analyse the primary motions.

To simulate the effects of gravity and muscular load, in vitro experiments often add an axial preload. As the nature and size of this preload is unknown in adolescent spines, we chose not to use such a preload. The effect of this choice was tested by comparing an adult model with and without $500 \mathrm{~N}$ axial preload to motion values reported in the literature.

\subsubsection{Average growth data for girls}

As severe scoliosis mainly occurs in girls, we used average geometrical growth data for girls between 10 and 14 years and between 10 and maturity as found in literature (table 4).

\begin{tabular}{|rccc|} 
Parameter & $\begin{array}{c}\text { Change } \\
\mathbf{1 0 y}-\mathbf{1 4 y}\end{array}$ & $\begin{array}{c}\text { Change } \\
\mathbf{1 0 y}-\mathbf{a d u l t}\end{array}$ & Ref. \\
\hline vertebral body height (VBH) & $+28.0 \%$ & $+41.0 \%$ & 29 \\
disc height (DH) & $+2.3 \%$ & $+3.3 \%$ & 30 \\
endplate width (EPW) & $+8.0 \%$ & $+10.0 \%$ & 29 \\
endplate depth (EPD) & $+18.2 \%$ & $+25.5 \%$ & 31 \\
nucleus size & $-10.0 \% *$ & $-14.0 \%$ & 32,33 \\
transverse processes width (TPW) & $+18.2 \%$ & $+25.5 \%$ & see text \\
spinous process length (SPL) & $+18.2 \%$ & $+25.5 \%$ & see text \\
ligament area & $+12.5 \%$ & $+18.3 \%$ & 34 \\
facet height (FH) & $+19.5 \%$ & $+28.0 \%$ & 35 \\
facet width (FW) & $+27.5 \%$ & $+38.0 \%$ & 35 \\
spinal canal depth (SCD) & $0.0 \%$ & $0.0 \%$ & 36 \\
spinal canal width (SCW) & $0.0 \%$ & $0.0 \%$ & 36 \\
inter facet width (IFW) & $0.0 \%$ & $0.0 \%$ & 37 \\
transverse facet angle (TFA) & $0.0 \%$ & $0.0 \%$ & 37 \\
longitudinal facet angle (LFA) & $0.0 \%$ & $0.0 \%$ & 37 \\
spinous process angle (SPA) & $0.0 \%$ & $0.0 \%$ & 37 \\
vertebra lordosis angle (VLA) & $0.0 \%$ & $0.0 \%$ & see text \\
disc lordosis angle (DLA) & $0.0 \%$ & $0.0 \%$ & see text \\
\hline *) interpolated & & & \\
\hline
\end{tabular}

Table 4: Geometrical change during growth spurt (10 years-adult) for girls as reported in literature.

As literature data was not available for transverse and spinous process growth, we assumed this growth to be related to the depth growth of the vertebrae; both grow through enchondral ossification combined with periosteal growth. Growth changes in vertebral and disc lordosis angle are assumed to be zero, because the change in lumbar lordosis during growth is less than $1^{\circ}$ per motion segment ${ }^{38-40}$. Changes in the facet width and height are combined into facet area changes.

Effects of changing mesh size due to growth-related remeshing were verified to be non-present. 


\subsubsection{Analysis of the stiffness}

All geometrical changes were effectuated in spinal models of a 10 and 14 year old girl. Stiffness's of these models were compared to that of the adult model.

Subsequently, in the10 year old model, each parameter was grown separately to determine its effect on spinal stiffness. The average stiffness for the range $0-10 \mathrm{Nm}$ of the 10 year old and the partially grown segments were compared.

\subsection{Results}

\subsubsection{Model validation against in vitro tests}

Results from the simulations of the intact motion segment with and without preload are within the range found in literature (figure 3 ).

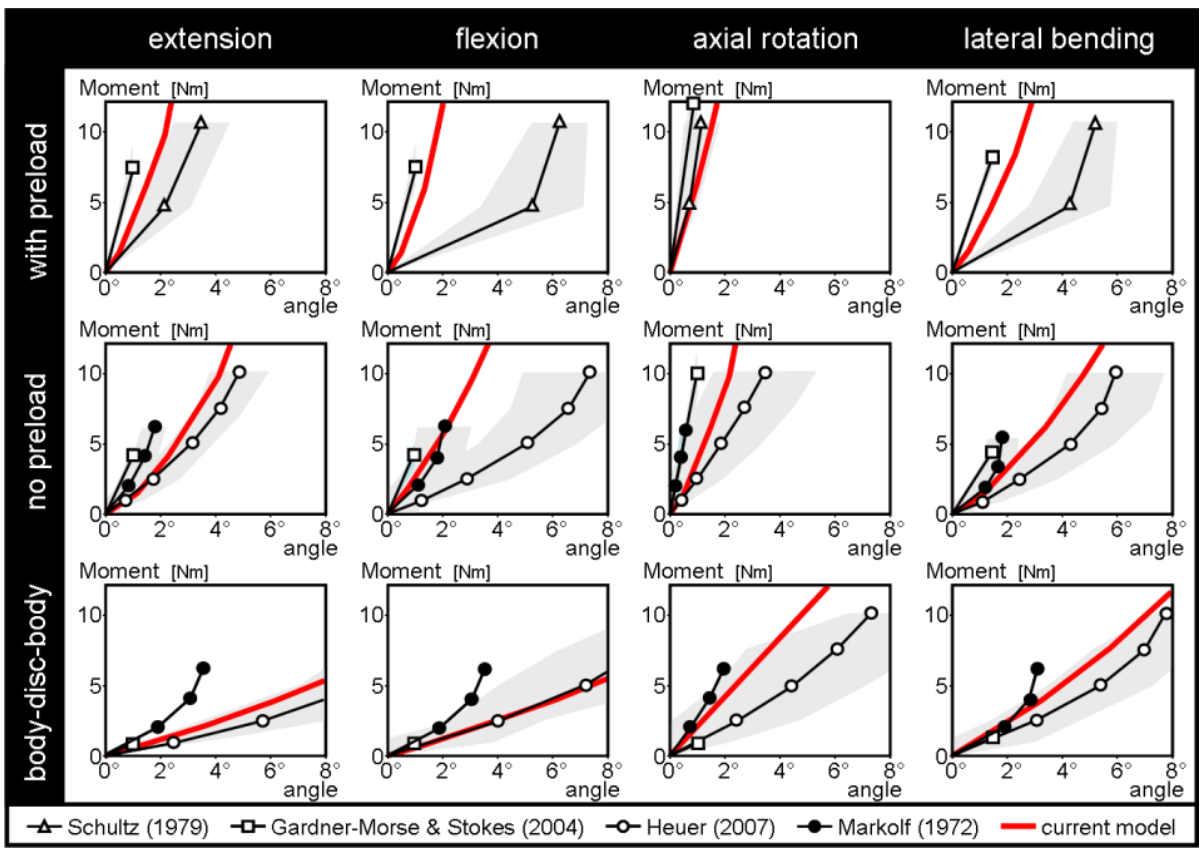

Figure 3: The model is validated with in vitro measurements from literature. Data points are presented by markers and standard deviation by the shaded areas. Three situations are compared: intact motion segment with preload, intact motion segment without preload and body-disc-body without preload.

The effect of the preload in the simulations is also consistent with literature findings: it increases stiffness and linearity of the load-displacement behaviour ${ }^{26}$. The behaviour of the body-disc-body without preload is also within the range provided in literature 
The effects of three-dimensional geometrical changes during adolescent growth on the biomechanics of a spinal motion segment

(figure 3) and the relative contributions of ligaments and facet joints are comparable to those reported in experiments (figure 4).

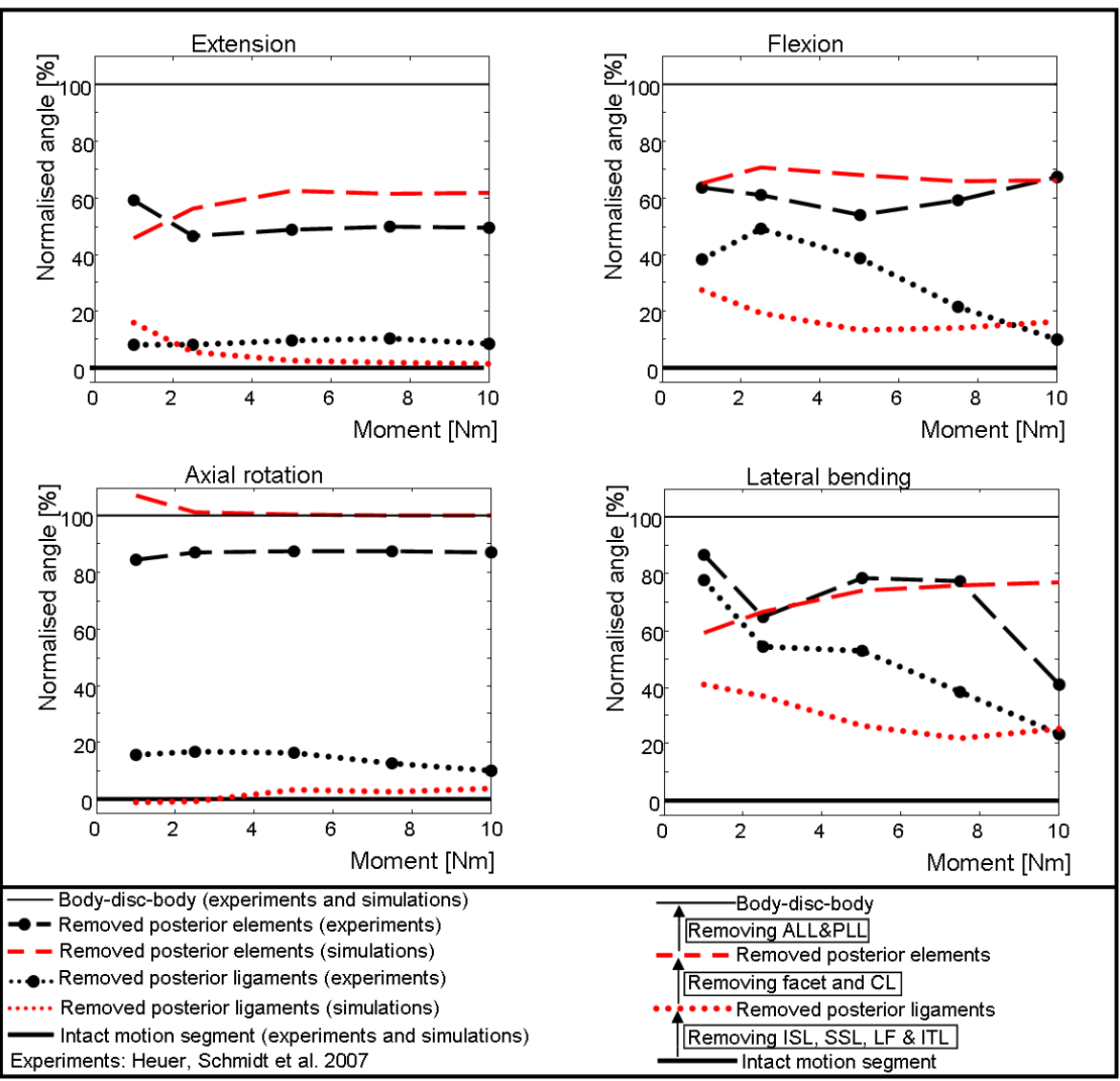

Figure 4: The relative contribution of the ligaments, facets and disc in the model is validated by comparing 2 reduced stages with similar in vitro experiments 27 . The results were normalised; for both the model and experimental data the difference in the range of motion between the intact and the body-disc-body segment were set at $100 \%$. 


\subsubsection{Effects of adolescent growth (combined parameter variation)}

The stiffness of the segment increases significantly during adolescence. The increase is largest for flexion (44\%) and smallest for lateral bending and extension $(36 \%)$. For all directions, the changes that occur between 10 and 14 years of age are about two thirds of the total change during the adolescent growth. The typical non-linear load-displacement behaviour does not change during adolescence (figure 5).
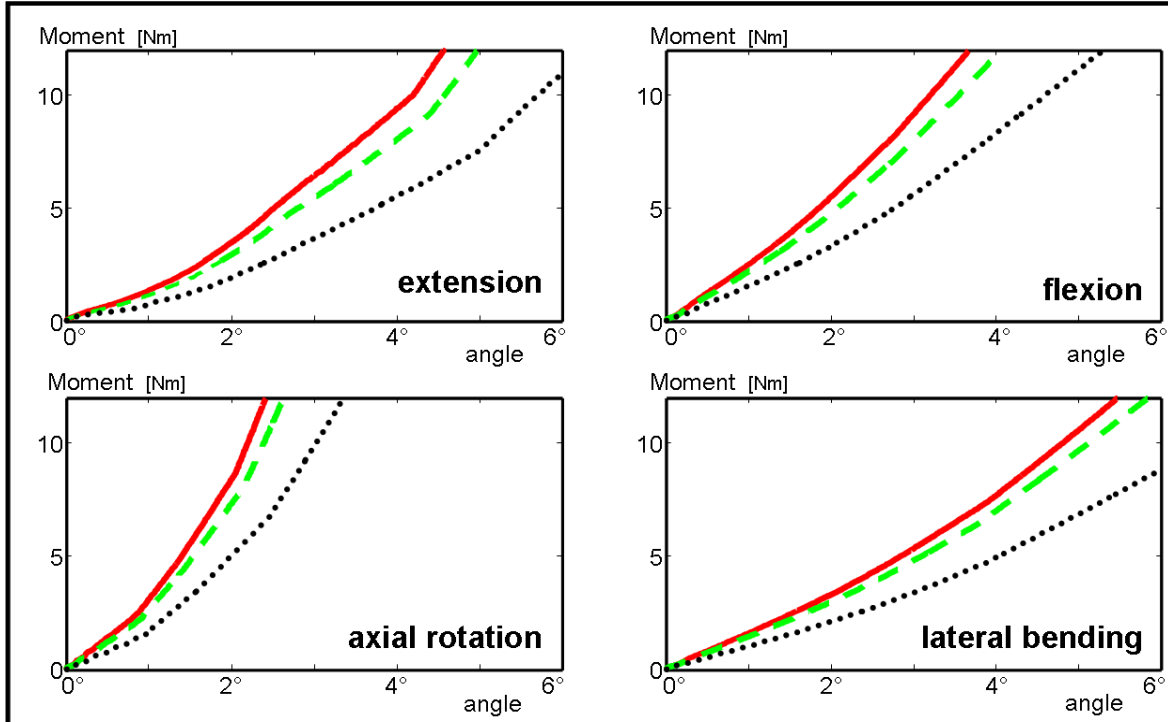

adult $\quad$ fourteen year

$\cdots$ ten year

Figure 5: The changing stiffness of a motion segment during adolescence: displacement for 10 year old, 14 year old and adult motion segment under extension, flexion, axial rotation and lateral bending load.

\subsubsection{Effects of single geometrical change (parameters varied separately)}

The results for single parameter changes (figure 6) show that the increase in endplate depth increases stiffness in all directions, while the increase in endplate width mainly increases lateral bending stiffness. The increase of discal and vertebral heights results in a decreased stiffness in all directions. The increased ligament area increases stiffness in all directions. The increased spinous process length only increases stiffness during flexion and axial rotation, while the increased transverse processes width only affects lateral bending stiffness. The effects of changes in facet area and nucleus size are negligible. 


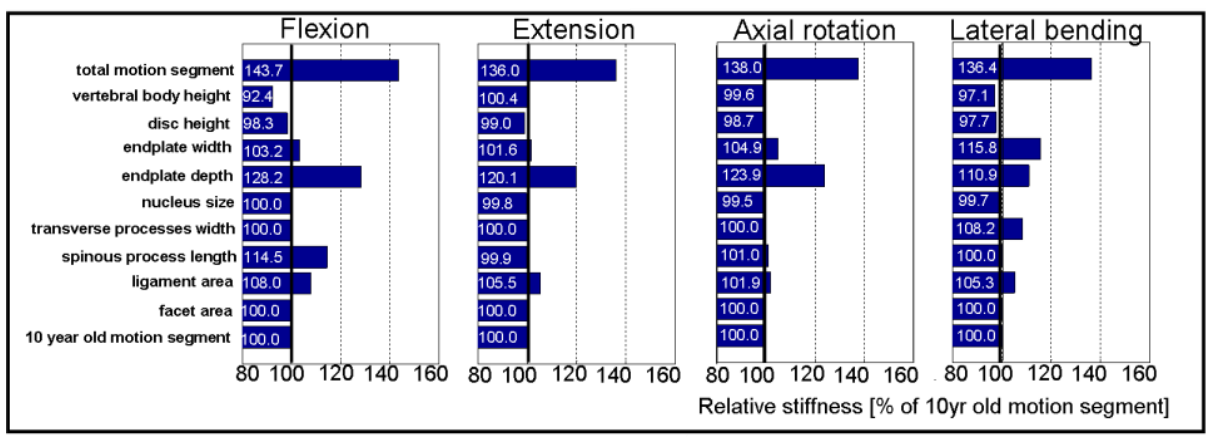

Figure 6: The effect of the geometrical growth change of single parameters on the average stiffness (for load ranging from 0 and $10 \mathrm{Nm}$ ) is expressed as percentage of the stiffness for a ten year old motion segment.

\subsection{Discussion}

We found that the stiffness of a L3-L4 motion segment increases by about $40 \%$ solely due to the geometry changes during adolescent growth. As no other study previously analysed the effects of adolescent growth on spinal stiffness, we can only relate our results to studies on paediatric growth. One modelling study ${ }^{11}$ predicted an increase in stiffness for flexion of $150 \%$ and extension of $375 \%$ between six years of age and maturity, based on changing geometry and mechanical properties. One experimental study ${ }^{41}$ measured spines of young children; other studies are used to make a comparison with adults ${ }^{42,43}$. This resulted in increasing stiffness of $40 \%$ for flexion and $110 \%$ for extension between five years of age and maturity. As expected, the increase in stiffness due to modelling geometrical adolescent growth is less than the published results for combined geometrical and mechanical changes between young children and adults.

For further understanding, this increase in stiffness can be divided into changes in the stiffness of the disc, ligaments and facet joints.

The changes of the stiffness of the disc can be explained by viewing the disc as a beam, where the bending stiffness is given by:

$\frac{\mathrm{E} * \mathrm{I}}{\mathrm{L}}$

(equation 2)

with the Young's modulus (E), the area moment of inertia (I) and the length of the beam (L).

This equation shows the limited (linear) effect of the disc height (=length of the beam) on the bending stiffness in each of the motion directions.

For an elliptic cross-sectional area the following formulas define the moments of inertia for the various movement directions: 


$$
\begin{array}{ll}
\mathrm{I}_{\text {flexion/exension }}=\frac{\pi *(\text { width }) *(\text { depth })^{3}}{64} & \\
\mathrm{I}_{\text {lateralbending }}=\frac{\pi *(\text { depth }) *(\text { width })^{3}}{64} & \text { (equation 3a to c) } \\
\mathrm{I}_{\text {axial rotation }}=\frac{\pi * \text { width } * \text { depth } *\left((\text { width })^{2}+(\text { depth })^{2}\right)}{64} &
\end{array}
$$

For flexion and extension, the endplate depth is thus the main contributor (third power); for lateral bending, endplate width is the main contributor (third power); and for axial rotation endplate width and depth contribute equally (although the larger growth increase in endplate depth does of course result in a larger increase in stiffness).

However, when the quantitative changes (table 4) are implemented in these equations, the calculated beam theory effect on the stiffness is larger than those predicted by the FE-simulations, particularly for axial rotation. This is due to the fact that the total behaviour of the motion segment is not only determined by the bending stiffness of the disc, but also by the stiffness of ligaments and facet joints.

The changes in ligament stiffness during growth are influenced by the changing ligament area, but also by the changing length of the spinous process and the width of the transverse processes, as they result in an increase in moment arm of the attaching ligaments. These effects are only present in the movement directions in which these ligaments are activated: those attached to the spinous process limit flexion and axial rotation, those attached to the transverse processes limit lateral bending. The stiffness decrease due to the increasing vertebral height can be explained by an increase of the length of the ligaments and thus decreased ligament stiffness.

The only geometry associated with the facet joints that changes during adolescence, the facet area, has a negligible effect on the stiffness. This implies that the absolute stiffness of the facet joints during adolescence does not change, although the relative contribution to the total motion segment stiffness does change.

This study has some limitations. Firstly, we used healthy growth data to study growth in scoliotic patients. However, studies that analysed possible effects of scoliosis on growth found no significant differences in neither height nor width of vertebrae inside and outside the scoliotic curve ${ }^{29}$ nor in three-dimensional trunk growth for a scoliotic and a healthy group ${ }^{44}$. It thus appears that adolescent growth of scoliotic girls is comparable to that of healthy girls, although girls with idiopathic scoliosis may have an earlier growth spurt than healthy girls ${ }^{45}$.

Secondly, the vertebrae were modelled as rigid bodies. It is known that vertebrae deform substantially under compression or moment loads above $10 \mathrm{Nm}^{46,47}$. As we studied moments up to $10 \mathrm{Nm}$, this should not affect our results much. Growth of the rigid vertebra (transverse processes width, spinous process length and vertebral body height) does changes ligament arm and stiffness. Based on our results these changes are expected to have a much larger effect on the stiffness of the motion segment than additional changes due to growth of deformable vertebrae. The rigid body assumption thus appears valid.

Thirdly, we studied only the geometrical changes during adolescent growth; changes in the mechanical properties were not included. As no literature data are available on 
the quantitative changes of biomechanical properties during growth, it is currently impossible to model these aspects. Further studies into these properties would be desirable, but may also be very difficult, because interpersonal variance is substantial and young donor spines are rare.

Fourthly and mainly, we used a lumbar motion segment while scoliosis is typically located in the thoracolumbar spine. This was done because insufficient literature was available to provide input data and validation for a thoracic model. By comparing differences in thoracic and lumbar motion segment behaviour and the regional differences during adolescent growth, we can hypothesize about the results of the growth in the thoracic spine. Especially the relative contributions of the various structures (ligaments, facet joints and disc) to the total motion segment stiffness are different for thoracic and lumbar region. When comparing the thoracic and lumbar ligaments, the halved ALL area is the most striking difference ${ }^{22}$. This results in a reduced contribution of the thoracic ligaments to the stiffness in extension. The more horizontal orientation of the thoracic facet joints will result in a higher resistance in extension and less contribution in axial rotation than in the lumbar region ${ }^{17}$. The relative contribution of the disc is deduced from comparison of body-disc-body with motion segment behaviour. In flexion, extension and lateral bending the relative contribution of the disc is similar, but in axial rotation it is larger for the thoracic region compared to the lumbar region ${ }^{24}$.

Although height growth of both vertebrae and discs are comparable for the lumbar and thoracic spine ${ }^{29,48,49}$, no quantitative data on growth of other dimensions in the thoracic region are reported. When we assume that growth in the thoracic spine is comparable to that in the lumbar spine, we can hypothesize about the effects of growth in the thoracic region. Especially the disc and ligament contributions appear of interest: the changes in facet joint geometry during adolescent growth seem to have little effect on the stiffness. As the relative growth and disc and ligament contributions to flexion and lateral bending stiffness are comparable for thoracic and lumbar motion segments, the stiffness increase during growth will likely also be comparable. In axial rotation, the relative contribution of the disc in the thoracic region is larger than in the lumbar region and therefore the effects of similar growth of the disc on the total motion segment stiffness is expected to be larger. In extension, the relative contribution and thus the effect of comparable growth of the disc on the total motion segment will likely be similar. However, the relative contribution of the ligaments is smaller, so total growth will presumably result in a smaller change in stiffness of the total motion segment for the thoracic region compared to the lumbar region in extension.

Furthermore, it is obvious that the ribcage significantly influences the behaviour of the thoracic spine. Because too little is known about the contribution of the ribcage to the spinal mechanics, let alone its changes during adolescent growth, only the behaviour of the spine was considered.

In this study, we compared average geometry changes. We suspect that in clinical practice the biomechanics of a patient's spine is also largely influenced by interpersonal variation in geometry. We are currently performing a study on interpersonal variations in geometry and the effects that those variations have on the biomechanics of the spine. 


\subsection{Conclusion}

We found that the stiffness of a lumbar spinal segment increases about $40 \%$ due to the changes in geometry related to adolescent growth. Although the height is the largest geometrical change during adolescent growth, its effect on the stiffness is small. The role of particularly endplate depth and width were found to be much larger. Hence, when analysing or modelling the biomechanics of the growing adolescent spine, the implementation of depth and width change, beside the usually implemented height change, is essential.

For the treatment of scoliosis, the key conclusion is that during adolescence the stiffness of the spine increases significantly. This will influence the correction provided by braces and implants used during adolescent growth and treatment planning should take this into account.

\section{Acknowledgement}

This study was financially supported by the Dutch Technology Foundation, STW, applied science division of NWO and the Technology Program of the Ministry of Economic Affairs.

We also thank ESI Group for the use of their software (PAM-CRASH) and Jos Boeije for his help in the pilot for this study.

\section{Conflict of interest statement}

None. 


\section{References}

[1] N.H. Miller, 'Causes and natural history of adolescent idiopathic scoliosis.'. Orthopedic Clinics of North America Vol. 30, pp. 343-352., 1999.

[2] J.W. Roach, 'Adolescent idiopathic scoliosis'. Orthopedic Clinics of North America Vol. 30, pp. 353-365, 1999.

[3] K.M.C. Cheung, K.D.K. Luk, 'Prediction of correction of scoliosis with use of the fulcrum bending radiograph'. J Bone Joint Surg Vol. 79-A, pp. 1144-1150, 1997.

[4] R. Vedantam, L.G. Lenke, K.H. Bridwell, D.L. Linville, 'Comparison of push-prone and lateral-bending radiographs for predicting postoperative coronal alignment in thoracolumbar and lumbar scoliotic curves.'. Spine Vol. 25, pp. 76-81, 2000.

[5] M.-E. Lamarre, S. Parent, H. Labelle, C.-E. Aubin, J. Joncas, A. Cabral, Y. Petit, 'Assessment of spinal flexibility in adolescent idiopathic scoliosis: suspension versus sidebending radiography.'. Spine Vol. 34, pp. 591-597, 2009.

[6] M. Troke, A.P. Moore, F.J. Maillardet, E. Cheek, 'A normative database of lumbar spine ranges of motion'. Manual Therapy Vol. 10, pp. 198-206., 2005.

[7] M. Kondratek, J. Krauss, C. Stiller, R. Olson, 'Normative Values for Active Lumbar Range of Motion in Children.'. Pediatric Physical Therapy Vol. 19, pp. 236-244., 2007.

[8] J. Carrier, C. Aubin, I. Villemure, H. Labelle, 'Biomechanical modelling of growth modulation following rib shortening or lengthening in adolescent idiopathic scoliosis'. Medical and Biological Engineering and Computing Vol. 42, pp. 541-548, 2004.

[9] I. Villemure, C.E. Aubin, J. Dansereau, H. Labelle, 'Simulation of progressive deformities in adolescent idiopathic scoliosis using a biomechanical model integrating vertebral growth modulation'. J Biomech Eng Vol. 124, pp. 784-790, 2002.

[10] I. Stokes, 'Analysis and simulation of progressive adolescent scoliosis by biomechanical growth modulation'. European Spine Journal Vol. 16, pp. 1621-1628, 2007.

[11] S. Kumaresan, N. Yoganandan, F.A. Pintar, D.J. Maiman, S. Kuppa, 'Biomechanical study of pediatric human cervical spine: a finite element approach.'. J Biomech Eng Vol. 122, pp. 60$71,2000$.

[12] A.P. Dooris, V.K. Goel, N.M. Grosland, L.G. Gilbertson, D.G. Wilder, 'Load-sharing between anterior and posterior elements in a lumbar motion segment implanted with an artificial disc'. Spine Vol. 26, pp. E122-E129, 2001.

[13] F. Lavaste, W. Skalli, S. Robin, R. Roy-Camille, C. Mazel, 'Three-dimensional geometrical and mechanical modelling of the lumbar spine'. J Biomech Vol. 25, pp. 1153-1164., 1992.

[14] V.K. Goel, B.T. Monroe, L.G. Gilbertson, P. Brinckman, 'Interlaminar shear stresses and laminae separation in a disc. Finite element analysis of the L3-L4 motion segment subjected to axial compressive loads'. Spine Vol. 20, pp. 689-698., 1995.

[15] M.M. Panjabi, V. Goel, T. Oxland, K. Takata, J. Duranceau, M. Krag, M. Price, 'Human lumbar vertebrae. Quantitative three-dimensional anatomy.'. Spine Vol. 17, pp. 299-306., 1992.

[16] M.F. Eijkelkamp On the development of an artificial intervertebral disc. PhD thesis, Rijksuniversiteit Groningen/ 2002.

[17] Y. Masharawi, B. Rothschild, G. Dar, S. Peleg, D. Robinson, E. Been, I. Hershkovitz, 'Facet orientation in the thorocolumbar spine. Three-dimensional anatomic and biomechanical analysis'. Spine Vol. 29, pp. 1755-1763., 2004.

[18] D.J.A. Heylings, 'Supraspinous and interspinous ligaments of the human lumbar spine'. Journal of Anatomy Vol. 125, pp. 127-131., 1978.

[19] A. Van der Plaats, A.G. Veldhuizen, G.J. Verkerke, 'Numerical simulations of asymmetrically altered growth as initiation mechanism of scoliosis.'. Annals of Biomedical Engineering Vol. 35, pp. 1206-1215., 2007.

[20] A.L. Nachemson, J.H. Evans, 'Some mechanical properties of the third human lumbar interlaminar ligament (ligamentum flavum)'. J Biomech Vol. 1, pp. 211, 1968.

[21] H. Tkaczuk, 'Tensile properties of human lumbar longitudinal ligaments.'. Acta Orthop

Scand Vol. 115 S, pp. 1-69., 1968. 
[22] J. Chazal, A. Tanguy, M. Bourges, G. Gaurel, G. Escande, M. Guillot, G. Vanneuville, 'Biomechanical properties of spinal ligaments and a histological study of the supraspinal ligament in traction'. J Biomech Vol. 18, pp. 167-176., 1985.

[23] J.S. Little, P.S. Khalsa, 'Material properties of the human lumbar facet joint capsule'. J Biomech Eng Vol. 127, pp. 15-24, 2005.

[24] K.L. Markolf, 'Deformation of the thoracolumbar intervertebral joints in response to external loads: a biomechanical study using autopsy material.'. J Bone Joint Surg Vol. 54-A, pp. 511 533, 1972.

[25] A. Schultz, D.N. Warwick, M.H. Berkson, A.L. Nachemson, 'Mechanical properties of human lumbar spine motion segments - Part I: responses in flexion, extension, lateral bending and torsion.'. J Biomech Eng Vol. 101, pp. 46-52, 1979.

[26] M.G. Gardner-Morse, I.A.F. Stokes, 'Structural behavior of human lumbar spinal motion segments'. J Biomech Vol. 37, pp. 205-212., 2004.

[27] F. Heuer, H. Schmidt, Z. Klezl, L. Claes, H.-J. Wilke, 'Stepwise reduction of functional spinal structures increase range of motion and change lordosis angle'. J Biomech Vol. 40, pp. 271-280., 2007.

[28] H.J. Wilke, K. Wenger, L. Claes, 'Testing criteria for spinal implants: recommendations for the standardization of in vitro stability testing of spinal implants'. European Spine Journal Vol. 7, pp. 148-154., 1998.

[29] A.G. Veldhuizen, P. Baas, P.J. Webb, 'Observations on the growth of the adolescent spine'. J Bone Joint Surg Vol. 68-B, pp. 724-728, 1986.

[30] I.A.F. Stokes, L. Windisch, 'Vertebral height growth predominates over intervertebral disc height growth in adolescents with scoliosis.'. Spine Vol. 31, pp. 1600-1604., 2006.

[31] M.E. Brandner, 'Normal values of the vertebral body and intervertebral disk index during growth.'. AJR Vol. 110, pp. 618-627, 1970.

[32] A. Peacock, 'Observations on postnatal structure of intervertebral disc in man'. Journal of Anatomy Vol. 86 pp. 162-179., 1956.

[33] A.A. White, M.M. Panjabi (1978) Clinical Biomechanics of the Spine. 1st edn. Lippincott Philadelphia, USA

[34] T.A.L. Wren, G.S. Beaupre, D.R. Carter, 'A model for loading-dependent growth, development, and adaptation of tendons and ligaments'. J Biomech Vol. 31, pp. 107-114., 1998.

[35] R. Miyake, T. Ikata, S. Katoh, T. Morita, 'Morphologic analysis of the facet joint in the immature lumbosacral spine with special reference to spondylolysis'. Spine Vol. 21, pp. 783789., 1996.

[36] R.D. Labrom, 'Growth and maturation of the spine from birth to adolescence'. $J$ Bone Joint Surg Vol. 89-A, pp. 3-7, 2007.

[37] S. Reichmann, 'The postnatal development of form and orientation of the lumbar intervertebral joint surfaces'. Anatomy and Embryology Vol. 133, pp. 102-123., 1971.

[38] S.A. Voutsinas, G.D. MacEwan, 'Sagittal profiles of the spine.'. Clin Orthop Relat Res Vol. 210, pp. 235-242., 1986.

[39] J.-M. Mac-Thiong, E. Berthonnaud, J.R. Dimar, R. Betz, H. Labelle, 'Sagittal alignement of the spine and pelvis during growth.'. Spine Vol. 29, pp. 1642-1647., 2004.

[40] C. Giglio, J. Volpon, 'Development and evaluation of thoracic kyphosis and lumbar lordosis during growth'. J Child Orthop Vol. 1, pp. 187-193, 2007.

[41] J. Ouyang, Q. Zhu, W. Zhao, Y. Xu, W.J. Chen, S. Zhong, 'Biomechanical assessment of the pediatric cervical spine under bending and tensile loading'. Spine Vol. 30, pp. E716-E723, 2005.

[42] K. Takeshita, E.T.K. Peterson, D. Bylski-Austrow, A.H. Crawford, K. Nakamura, 'The nuchal ligamnet restrains cervical spine flexion.'. Spine Vol. 29, pp. E388-E393., 2004.

[43] R.D. Rao, M. Wang, L.M. McGrady, T.J. Perlewitz, K.S. David, 'Does anterior plating of the cervical spine predispose to adjacent segment changes? '. Spine Vol. 30, pp. 2788-2792, 2005. [44] Y.P. Charles, A. Diméglio, M. Marcoul, J.-F. Bourgin, A. Marcoul, M.-C. Bozonnat, 'Influence of idiopathic scoliosis on three-dimensional thoracic growth'. Spine Vol. 33, pp. 12091218., 2008. 
The effects of three-dimensional geometrical changes during adolescent growth on the biomechanics of a spinal motion segment

[45] G. Hagglund, J. Karlberg, S. Willner, 'Growth in girls with adolescent idiopathic scoliosis'. Spine Vol. 17, pp. 108-111., 1992.

[46] A. Shirazi-Adl, 'Analysis of role of bone compliance on mechanics of a lumbar motion segment.'. J Biomech Eng Vol. 116, pp. 408-412, 1994.

[47] J.J. MacLean, J.P. Owen, J.C. latridis, 'Role of endplates in contributing to compression behaviors of motion segments and intervertebral discs'. J Biomech Vol. 40, pp. 55-63, 2007.

[48] J.R. Taylor, 'Growth of human intervertebral discs and vertebral bodies'. Journal of Anatomy Vol. 120, pp. 49-68., 1975.

[49] J.F. Sarwark, C.-E. Aubin, 'Growth considerations of the immature spine'. J Bone Joint Surg Vol. 89-A, pp. 8-13, 2007. 



\section{Chapter 4}

Influence of interpersonal geometrical variation on spinal motion segment stiffness - implications for patient-specific modelling

G.J.M. Meijer, J. Homminga, A.G. Veldhuizen, G.J. Verkerke

Spine v. 36(14): pp. E929-935, 2011 


\section{Abstract}

\section{Study design}

A validated finite element model of an L3-L4 motion segment is used to analyse the effects of interpersonal differences in geometry on spinal stiffness.

\section{Objective}

The objective of this study is to determine which of the interpersonal variations of the geometry of the spine have a large effect on spinal stiffness. This will improve patient-specific modelling.

\section{Summary of background Data}

The parameters that define the geometry of a motion segment are vertebral height, disc height, endplate width, endplate depth, spinous process length, transverse process width, nucleus size, lordosis angle, facet area, facet orientation, and the cross-sectional areas of the ligaments. All these parameters differ between patients. The influence of each parameter on spinal stiffness is largely unknown and such knowledge would greatly help in patient-specific modelling of the spine.

\section{Methods}

The range of interpersonal variation of each of the geometric parameters was set at mean $\pm 2 S D$ (covering $95 \%$ of the population). Subsequently we determined the effect of each of these ranges on the bending stiffness in flexion, extension, axial rotation and lateral bending.

\section{Results}

Disc height had the largest influence; a maximal disc height reduced the spinal stiffness to $75-86 \%$ of the mean motion segment stiffness, while a minimal disc height increased the spinal stiffness to $154-226 \%$ of the mean motion segment stiffness. Lordosis angle, transversal and longitudinal facet angle, endplate depth and area of the capsular ligament also had a substantial influence $(>5 \%)$ on the stiffness, but considerable less than the influence of the disc height. Ligament areas, nucleus size, spinous process length and length of processes are of negligible effect $(<2 \%)$ on the stiffness.

\section{Conclusion}

The disc height should be accurately determined in patients to estimate the spinal stiffness. Ligament areas, nucleus size, spinous process length and transverse process width do not need patient-specific modelling. 


\subsection{Introduction}

For the outcome of spinal deformity surgery, the stiffness of the patient's spine plays a large role and is therefore often used in surgical planning strategies. In adolescent scoliosis correction the stiffness of the curve is used to predict the optimal surgical approach, the number of vertebrae to be instrumented and the outcome of the correction ${ }^{1,2}$. Because every patient is unique and the interpersonal variation in spinal stiffness is large, the resulting strategy has to be optimized for a specific patient. So a method to determine the spinal stiffness in vivo and non-invasively would therefore be most welcome. In literature, a number of stiffness tests have been proposed, including radiographs in side bending ${ }^{3-5}$, fulcrum bending ${ }^{4-6}$, and traction

1,3,5. A common drawback of these methods is their sensitivity to muscle activation and support of the patient, which makes the test irreproducible and the results difficult to interpret ${ }^{7,8}$. More recently, traction radiographs made under general anaesthesia have been introduced ${ }^{5,9}$. Although this reduces the effects of muscle contraction, a new problem occurs; the anaesthesia makes it necessary to perform the test just before surgery and this is too late for an effective and convenient surgical planning. Most important however, all presented methods are analysing mobility of the spine rather than stiffness or flexibility, because forces involved are not considered. Prediction of the surgical outcome, based on applied forces and moments, is therefore not straightforward.

A possible solution to this problem may lie in the mechanical modelling of a patient's spine. To estimate patient-specific mechanical behaviour, patient-specific modelling of both geometry and material properties are necessary. Although side-bending radiographs are sometimes used to personalize mechanical properties, these mechanical properties actually are a result of the combined effects of patient-specific material properties and geometrical properties. A (linear) correction of the stiffness based on a single in vivo bending test, cannot capture the non-linear effects that occur due to large deformities (common in spinal deformities and their correction). Because there is no method for estimating in vivo material properties yet, and therefore implication in patient-specific modelling is unlikely, we will focus on the patient-specific geometry in this study.

Although the use of patient-specific geometry in pre-operative planning is rather common ${ }^{7-9}$, the actual influence of this patient-specific geometry on the spinal stiffness is unknown. Some previous studies have analysed the influence of certain geometrical changes on mechanical behaviour of lumbar motion segments. Robin et al. ${ }^{10}$ studied the effect of a $5 \%$ change in the geometrical parameters on the spinal stiffness. They found a large effect of disc height, endplate width and endplate depth. The effects of spinous process length and vertebral height were found to be negligible. Natarjan et al. ${ }^{11}$ also found a large effect for the influence of disc height on motion segment stiffness and a (smaller) effect of the disc cross-sectional area. Lu et al. ${ }^{12}$ studied the influence of compression loads on a motion segment without posterior elements or ligaments, and also found a large influence of the disc height on the stiffness. Unfortunately, none of these studies analysed the effects of the physiological range of interpersonal geometry variance seen in real patients. Analysing the effects of this physiological range on the stiffness will show which geometrical parameters are the main determinants of interpersonal difference in 
patient-specific stiffness. These parameters therefore need an accurate determination in patient-specific modelling.

In this study we set out to determine the effects that the known range in interpersonal variance of spinal geometry has on the spinal stiffness. By using a validated finite element model (FE model) of a spinal motion segment, we were able to study the separate effects of the geometric parameters on spinal stiffness. These results can be used to optimize patient-specific modelling.

\subsection{Materials and methods}

For this study we used a previously developed and validated finite element model of an L3-L4 motion segment ${ }^{13}$ (figure 1).

This motion segment was based on mean geometric parameters and referred to as the mean motion segment.

As the spine's flexibility is dominated by the disc, the vertebrae were modelled as rigid bodies, while disc and ligaments are represented by a deformable mesh. The geometry of the disc and vertebrae was based on parameters from literature ${ }^{14-16}$. An overview of the mechanical properties ${ }^{17-21}$ is presented in table 1.

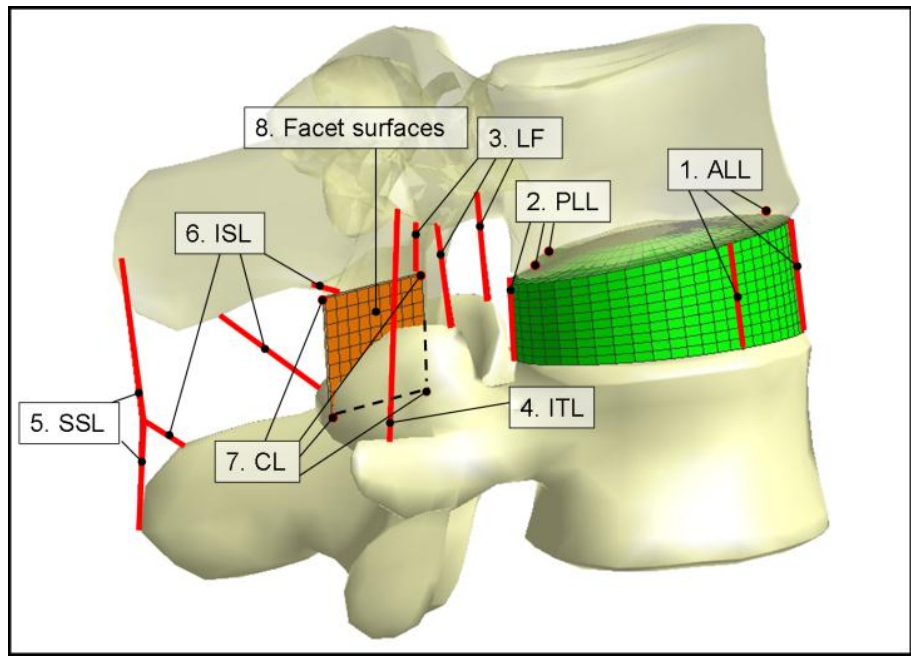

Figure 1: Finite Element model of L3-L4 motion segment

The intervertebral disc consisted of an incompressible nucleus (occupying $40 \%$ of the midsagittal cross-sectional area of the disc) and an annulus which contains fibres oriented at $+30^{\circ}$ and $-30^{\circ}$ to the transverse plane (volume ratio=16\%). All spinal ligaments - anterior longitudinal ligament (ALL), posterior longitudinal ligament (PLL), ligament flavum (LF), interspinous ligament (ISL), supraspinous ligament (SSL), intertransverse ligaments (ITL) and capsular ligaments (CL) - were included. The non-linear behaviour of the ligaments was implemented by tri-linear elastic behaviour. 


$\begin{array}{|rlc|}\text { Structure } & \text { Material properties } & \text { Ref } \\ \text { Nucleus } & \mathrm{E}=1 \mathrm{MPa}, \mathrm{v}=0.495 & 18 \\ \text { Annulus matrix } & \mathrm{E}=2 \mathrm{MPa}, \mathrm{v}=0.45 & 19 \\ \text { Annulus fibres } & \mathrm{E}=450 \mathrm{MPa} & 20 \\ \text { ALL } & \mathrm{k}_{1}=52 \mathrm{~N} / \mathrm{mm}(\varepsilon<12 \%) & \\ & \mathrm{k}_{2}=129 \mathrm{~N} / \mathrm{mm}(12 \%<\varepsilon<45 \%) & 20 \\ & \mathrm{k}_{3}=65 \mathrm{~N} / \mathrm{mm}(\varepsilon>45 \%) & \\ \text { PLL } & \mathrm{k}_{1}=50 \mathrm{~N} / \mathrm{mm}(\varepsilon<9 \%) & \\ & \mathrm{k}_{2}=118 \mathrm{~N} / \mathrm{mm}(9 \%<\varepsilon<34 \%) & \\ & \mathrm{k}_{3}=38 \mathrm{~N} / \mathrm{mm}(\varepsilon>34 \%) & \\ \text { LF } & \mathrm{k}_{1}=65 \mathrm{~N} / \mathrm{mm}(\varepsilon<5 \%) & \\ & \mathrm{k}_{2}=85 \mathrm{~N} / \mathrm{mm}(5 \%<\varepsilon<50 \%) & \\ & \mathrm{k}_{3}=26 \mathrm{~N} / \mathrm{mm}(\varepsilon>50 \%) & \\ \text { ISL } & \mathrm{k}_{1}=24 \mathrm{~N} / \mathrm{mm}(\varepsilon<12 \%) & \\ & \mathrm{k}_{2}=53 \mathrm{~N} / \mathrm{mm}(12 \%<\varepsilon<30 \%) & \\ & \mathrm{k}_{3}=25 \mathrm{~N} / \mathrm{mm}(\varepsilon>30 \%) & \\ \text { SSL } & \mathrm{k}_{1}=18 \mathrm{~N} / \mathrm{mm}(\varepsilon<12 \%) & \\ & \mathrm{k}_{2}=40 \mathrm{~N} / \mathrm{mm}(12 \%<\varepsilon<30 \%) & \\ & \mathrm{k}_{3}=19 \mathrm{~N} / \mathrm{mm}(\varepsilon>30 \%) & \\ \text { ITL } & \mathrm{k}_{1}=28 \mathrm{~N} / \mathrm{mm}(\varepsilon<9 \%) & \\ & \mathrm{k}_{2}=137 \mathrm{~N} / \mathrm{mm}(9 \%<\varepsilon<15 \%) & \\ & \mathrm{k}_{3}=46 \mathrm{~N} / \mathrm{mm}(\varepsilon>15 \%) & \\ \text { Facet surfaces } & \mathrm{k}_{1}=18 \mathrm{~N} / \mathrm{mm}(\varepsilon<100 \%) & \\ \text { Vertebrae } & \mathrm{k}_{2}=45 \mathrm{~N} / \mathrm{mm}(100 \%<\varepsilon<200 \%) & \mathrm{Rigid} \text { bodies } \\ & \mathrm{k}_{3}=15 \mathrm{~N} / \mathrm{mm}(\varepsilon>200 \%) & \\ & & \end{array}$

Table 1: Overview of material properties

The facet articular surfaces were modelled as parallel planes with an initial gap of 0.6 $\mathrm{mm}$. The non-linear stiffness of the cartilage is implemented in the contact definition of the surfaces. For all FE simulations PAM-CRASH software was used (ESI-group, France).

In this mechanical model, various geometric parameters were varied: vertebral height, disc height, endplate width, endplate depth, spinous process length, transverse process length, nucleus size, lordosis angle (combination of disc wedge angle and vertebral wedge angle), facet area, transverse facet angle, longitudinal facet angle and the cross-sectional areas of the ligaments (ALL, PLL, LF, ISL, ISL, SSL, ITL, CL). The physiological range of these geometrical parameters was obtained from literature ${ }^{14-16,20,22}$ as mean $\pm 2 S D$ and is presented in table 2 . The range is covering $95 \%$ of the population when a normal distribution is assumed.

Exception to this method are the ligament areas, which are based on modelling studies (area of ISL, SSL and CL) or experimental studies providing no SD due to limited sample numbers (area of LF and ITL). For these ligament areas, several studies in specimen without history of spine pathology are used to determine the interpersonal variation ${ }^{23-25}$. Because only lumbar data did not provide enough information, also thoracic and cervical studies had to be included. Regional differences in area are compensated for by calculating the SD as a percentage of the mean area. By taking the weighted mean, the difference in sample size is compensated for. 
For the determination of the orientation of the facet angle, the variations of the longitudinal and transverse angle are not completely independent, which means that the minimum value of the longitudinal and transverse angle had to be adapted slightly.

\begin{tabular}{|c|c|c|c|}
\hline Parameter & mean & SD & Ref. \\
\hline vertebra height $[\mathrm{mm}]$ & 24.1 & 1.1 & \\
\hline transverse processes width [mm] & 79.4 & 3.8 & 14 \\
\hline spinous process length $[\mathrm{mm}]$ & 70.1 & 1.2 & 14 \\
\hline spinal canal depth [mm] & 18.6 & 0.7 & 14 \\
\hline spinal canal width $[\mathrm{mm}]$ & 25.4 & 0.5 & 14 \\
\hline endplate width [mm] & 46.6 & 1.2 & 14 \\
\hline endplate depth [mm] & 35.5 & 0.9 & 14 \\
\hline disc height $[\mathrm{mm}]$ & 10 & 3.2 & 15 \\
\hline nucleus size $[\%]$ & $40 \%$ & $5 \%$ & 22 \\
\hline disc wedge angle $\left[{ }^{\circ}\right]$ & 7.5 & 2.5 & 15 \\
\hline vertebra wedge angle $\left[{ }^{\circ}\right]$ & 7.4 & 1.8 & 14 \\
\hline ALL area $\left[\mathrm{mm}^{2}\right]$ & 66 & 12.5 & 20 \\
\hline PLL area $\left[\mathrm{mm}^{2}\right]$ & 26 & 6.9 & 20 \\
\hline $\mathrm{LF}$ area $\left[\mathrm{mm}^{2}\right]$ & 39 & 6.6 & See text \\
\hline ISL area $\left[\mathrm{mm}^{2}\right]$ & 40 & 12 & See text \\
\hline SSL area $\left[\mathrm{mm}^{2}\right]$ & 30 & 16.8 & See text \\
\hline ITL area $\left[\mathrm{mm}^{2}\right]$ & 2 & 0.2 & See text \\
\hline CL area $\left[\mathrm{mm}^{2}\right]$ & 30 & 12.6 & See text \\
\hline facet height $[\mathrm{mm}]$ & 16.7 & 0.6 & 14 \\
\hline facet width [mm] & 14.7 & 0.8 & 14 \\
\hline facet transverse angle $\left[{ }^{\circ}\right]$ & 38.2 & 14.9 & 16 \\
\hline facet longitudinal angle $\left[{ }^{\circ}\right]$ & 170.1 & 8.3 & 16 \\
\hline
\end{tabular}

Table 2: Geometrical parameters from literature (mean and SD)

For each of these parameters, its effect on the bending stiffness in flexion, extension, axial rotation, and lateral bending was determined by changing only this parameter. We analysed loads from $0-10 \mathrm{Nm}$, by fixating the lower vertebra and applying pure moments to the upper vertebra. The rotation of the upper vertebra was used to analyse the primary motions. For both the minimum and the maximum value of the geometrical parameters, the average stiffness for the load range $(0-10 \mathrm{Nm})$ is compared to the average stiffness for the mean motion segment.

\subsection{Results}

The stiffness for the physiological minimal and maximal value of every geometric parameter is expressed as a percentage of the stiffness of the mean motion segment (table 3 and figure 2). The effect of physiologically occurring variance in geometry on the stiffness of the motion segment is negligible (less than $2 \%$ ) or low (between $2 \%$ and $5 \%$ ) for most geometric parameters.

For all loading directions disc height had the largest influences: ranging from a $14 \%$ $25 \%$ decrease of stiffness for the maximal disc height to a $54 \%-126 \%$ increase of stiffness for the minimal disc height. Capsular ligament area also significantly influenced the stiffness in all loading directions: ranging from a $13 \%-21 \%$ decrease for the minimal $\mathrm{CL}$ area to an $11 \%-26 \%$ increase for the maximum $\mathrm{CL}$ area. In 
Influence of interpersonal geometrical variation on spinal motion segment stiffness implications for patient-specific modelling

\begin{tabular}{|c|c|c|c|c|c|c|c|c|}
\hline Geometry parameter & \multicolumn{2}{|c|}{$\begin{array}{l}\text { Flexion } \\
\text { stiffness }\end{array}$} & \multicolumn{2}{|c|}{$\begin{array}{l}\text { Extension } \\
\text { stiffness }\end{array}$} & \multicolumn{2}{|c|}{$\begin{array}{l}\text { Lateral bending } \\
\text { stiffness }\end{array}$} & \multicolumn{2}{|c|}{$\begin{array}{l}\text { Axial rotation } \\
\text { stiffness }\end{array}$} \\
\hline & Min. & Max. & Min. & Max. & Min. & Max. & Min. & Max. \\
\hline vertebra height & $+3 \%$ & $-2 \%$ & $-0 \%$ & $+0 \%$ & $+1 \%$ & $-1 \%$ & $+0 \%$ & $-0 \%$ \\
\hline disc height & $+67 \%$ & $-24 \%$ & $+54 \%$ & $-14 \%$ & $+126 \%$ & $-24 \%$ & $+73 \%$ & $-25 \%$ \\
\hline endplate width & $-2 \%$ & $-1 \%$ & $-1 \%$ & $-0 \%$ & $-8 \%$ & $+7 \%$ & $-6 \%$ & $+4 \%$ \\
\hline endplate depth & $-9 \%$ & $+10 \%$ & $-5 \%$ & $+7 \%$ & $-3 \%$ & $+3 \%$ & $-6 \%$ & $+6 \%$ \\
\hline nucleus size & $-0 \%$ & $-0 \%$ & $+0 \%$ & $-1 \%$ & $+0 \%$ & $-2 \%$ & $+0 \%$ & $-2 \%$ \\
\hline $\begin{array}{r}\text { transverse processes } \\
\text { width }\end{array}$ & $-0 \%$ & $-0 \%$ & $+0 \%$ & $+0 \%$ & $-3 \%$ & $+3 \%$ & $-0 \%$ & $+0 \%$ \\
\hline spinous process length & $-3 \%$ & $+3 \%$ & $+0 \%$ & $+0 \%$ & $+0 \%$ & $+0 \%$ & $-0 \%$ & $+0 \%$ \\
\hline spinal canal depth & $-3 \%$ & $+3 \%$ & $-2 \%$ & $+3 \%$ & $+0 \%$ & $-0 \%$ & $-2 \%$ & $+2 \%$ \\
\hline spinal canal width & $-0 \%$ & $+0 \%$ & $+0 \%$ & $-0 \%$ & $-0 \%$ & $+0 \%$ & $-0 \%$ & $+0 \%$ \\
\hline lordosis angle & $+1 \%$ & $+2 \%$ & $+11 \%$ & $-9 \%$ & $+10 \%$ & $-9 \%$ & $+6 \%$ & $+16 \%$ \\
\hline ALL area & $-1 \%$ & $+1 \%$ & $-3 \%$ & $+3 \%$ & $-1 \%$ & $+1 \%$ & $-0 \%$ & $+0 \%$ \\
\hline PLL area & $-0 \%$ & $+1 \%$ & $-0 \%$ & $+0 \%$ & $-1 \%$ & $+1 \%$ & $-1 \%$ & $+1 \%$ \\
\hline LF area & $-3 \%$ & $+3 \%$ & $-0 \%$ & $-0 \%$ & $-0 \%$ & $+0 \%$ & $-1 \%$ & $+1 \%$ \\
\hline ISL area & $-2 \%$ & $+2 \%$ & $-0 \%$ & $+0 \%$ & $+0 \%$ & $+0 \%$ & $-0 \%$ & $+0 \%$ \\
\hline SSL area & $-14 \%$ & $+12 \%$ & $-1 \%$ & $+1 \%$ & $+0 \%$ & $+0 \%$ & $-1 \%$ & $+1 \%$ \\
\hline ITL area & $+0 \%$ & $+0 \%$ & $-0 \%$ & $+0 \%$ & $-2 \%$ & $+1 \%$ & $-1 \%$ & $+1 \%$ \\
\hline CL area & $-17 \%$ & $+13 \%$ & $-21 \%$ & $+26 \%$ & $-13 \%$ & $+11 \%$ & $-13 \%$ & $+11 \%$ \\
\hline facet area & $-0 \%$ & $+0 \%$ & $-1 \%$ & $+2 \%$ & $-1 \%$ & $+1 \%$ & $-1 \%$ & $+1 \%$ \\
\hline facet transverse angle & $+1 \%$ & $-1 \%$ & $-4 \%$ & $+3 \%$ & $-5 \%$ & $+9 \%$ & $-2 \%$ & $+1 \%$ \\
\hline facet longitudinal angle & $+3 \%$ & $-3 \%$ & $+38 \%$ & $-3 \%$ & $+2 \%$ & $-3 \%$ & $-10 \%$ & $+14 \%$ \\
\hline
\end{tabular}

Table 3: Change of motion segment stiffness by applying minimum (Min.) and maximum (Max.) geometry parameter values. The change is relative to the stiffness for mean parameter values, negative values indicating a decrease and positive values indicating an increase of the stiffness.

flexion, extension and axial rotation, a large effect was found for changing the endplate depth (5-9\% decrease for minimal depth; 6-10\% increase for maximum depth). In lateral bending and axial rotation, on the other hand, the endplate width has a large effect on the stiffness $(6-8 \%$ decrease for minimal width and $4-7 \%$ increase for maximal width). The lordosis angle had large effects on extension, lateral bending and axial rotation stiffness (6-11\% increase for the maximum lordosis angle; $9 \%$ decrease to $16 \%$ increase for the minimal lordosis angle). The longitudinal facet orientation influenced extension and axial rotation stiffness (3\% decrease and $14 \%$ increase for the maximum longitudinal facet angle respectively; $38 \%$ increase and $10 \%$ decrease for the minimal lordosis angle respectively), while the facet transverse orientation influenced the lateral bending stiffness $(9 \%$ increase for the maximum transverse facet angle and $5 \%$ decrease for the minimal lordosis angle). In flexion, large effects were also seen for and the SSL area $(12 \%$ increase for maximum area and $14 \%$ decrease for minimum area). 
Influence of interpersonal geometrical variation on spinal motion segment stiffness implications for patient-specific modelling

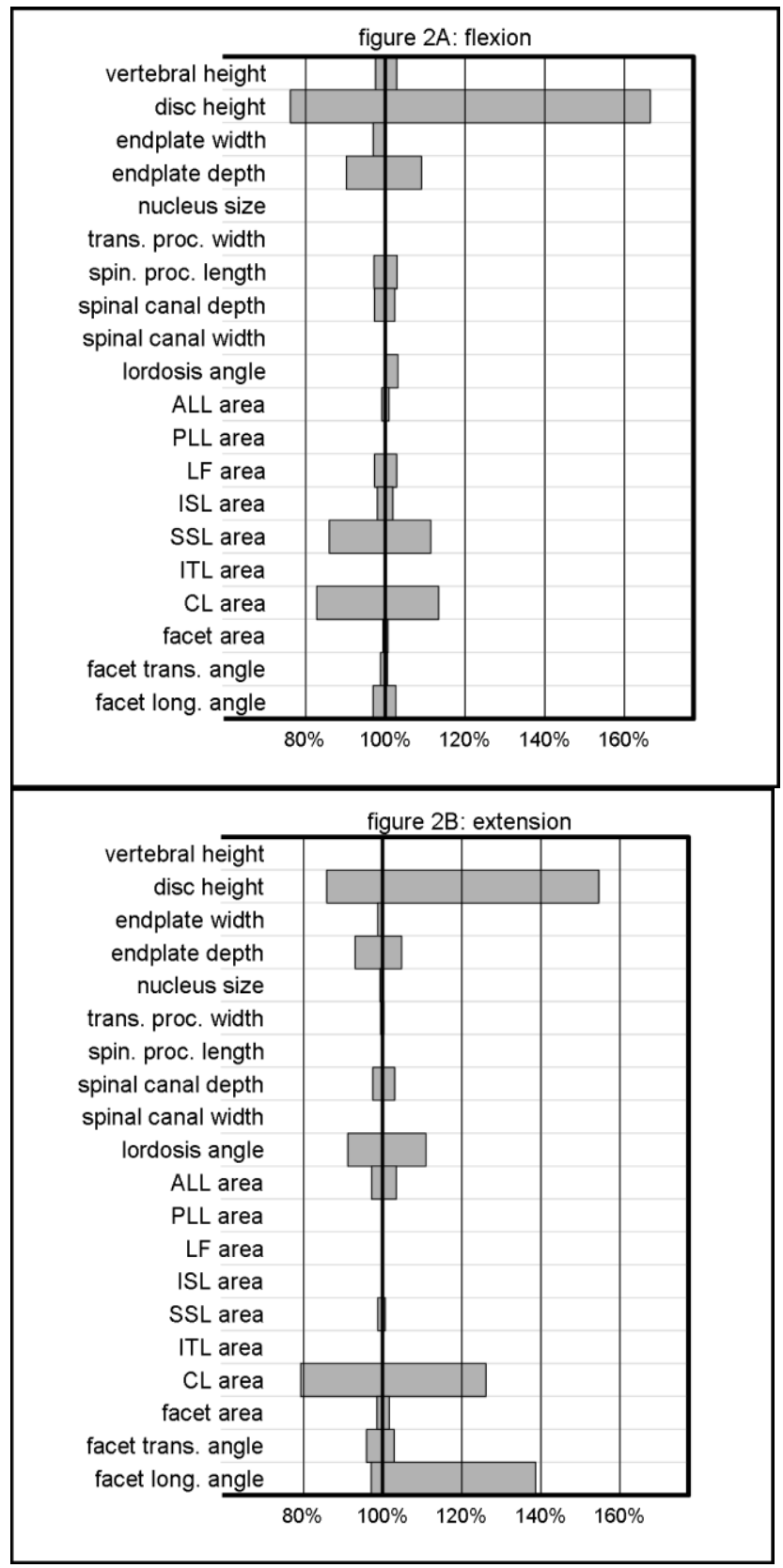

Figure 2: Effect of interpersonal geometry variation on spinal motion segment stiffness in A) Flexion, B) Extension, C) Lateral bending D) Axial rotation 
Influence of interpersonal geometrical variation on spinal motion segment stiffness implications for patient-specific modelling

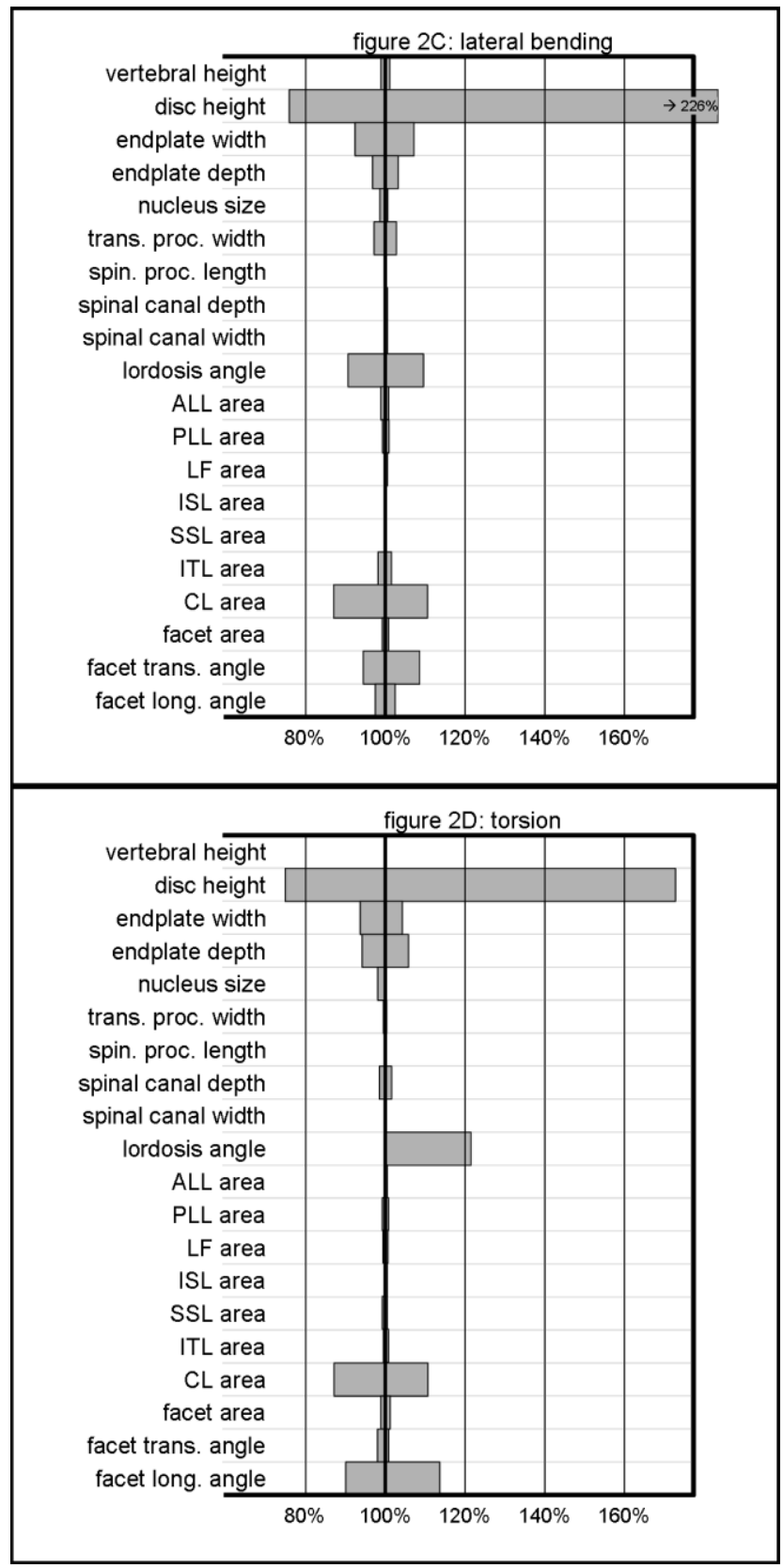

Figure 2: Effect of interpersonal geometry variation on spinal motion segment stiffness in A) Flexion, B) Extension, C) Lateral bending D) Axial rotation 


\subsection{Discussion}

In this study, we analysed the effects of the physiological range of geometrical dimensions on the stiffness of a motion segment. Our results show that the natural variance in vertebral height, spinous process length, transverse process length, nucleus size, facet area, and the cross-sectional areas of most ligaments (anterior longitudinal ligament, posterior longitudinal ligament, ligamentum flavum, interspinous ligament, and intertransverse ligament) has little influence on the stiffness of the motion segment. For these geometrical parameters the use of mean values appears acceptable for patient-specific models. Endplate depth and endplate width, lordosis angle, facet joint orientation, and the cross-sectional area of the capsular ligament and supraspinous ligament are of more importance to spinal stiffness. Endplate width, endplate depth and lordosis angle can be determined from $x$-ray images or CT scans. Facet joint angle and the cross sectional areas of the capsular ligament and supraspinous ligament are very difficult to determine in patients. These parameters may need MRI images, although even those may be of too low a resolution. However, the largest determinant of a patient's spinal stiffness by far is disc height. The disc height needs therefore accurate determination in patient-specific modelling. Fortunately this parameter can be easily and accurately determined from $\mathrm{x}$-rays or CT-scans. With this study we demonstrate that overall scaling makes no sense, because the disc height has a larger influence on the stiffness than the vertebral height. Although the total height of the patient thus has a large influence, the patient-specific ratio between disc height and vertebra height is even more important.

The relative high influence of the capsular ligament area can also be seen as a warning for damaging the capsular ligaments of the adjacent segments in fusion or all segments in non-fusion treatments: this will highly reduce the stiffness in all motion directions.

Our results compare well with previously published studies. Three studies ${ }^{10-12}$ show a decrease of the stiffness with increasing disc height, which we found as well. Natarajan and Andersson ${ }^{11}$ found that an increase of the endplate size results in an increase of the stiffness (except for torsion loads, where the role of facet joints dominates). This compares well to the effects we found for endplate width and depth. Robin et al ${ }^{10}$ found negligible effects for changing vertebral height and spinous process length, which is also what we found in this study.

In the effects of the physiological variation in geometrical parameters on spinal stiffness, two factors are important: firstly the actual variance that physiologically exists in that parameter and secondly the sensitivity of spinal stiffness to that parameter. A large effect on the stiffness due to the high physiological variance was for example observed for the influence of the area of the capsular ligaments, where the physiological variance is $84 \%$ and the effect on the stiffness is between $11 \%$ and $26 \%$ for the various bending directions.

A large effect on the stiffness due to a high sensitivity can for example be seen for the reduction of the disc height where the variance is $64 \%$ and the effect on the stiffness is between $67 \%$ and $126 \%$ for the various bending directions. 
This study has four main limitations. Firstly, in reality geometrical parameters will not show an independent variation, a certain dependency between the various parameters will exist. A patient is for example unlikely to combine a large endplate depth with a small endplate width. The ranges of stiffness presented in this study are therefore purely theoretical and clinical results will show a larger diversity, due to a combination of the various effects.

Secondly, although the focus of this study is on the application for scoliotic patients, the actual model is based on a healthy lumbar spine. In literature, no accurate geometrical parameters on the scoliotic spine are available, let alone the physiological range of interpersonal geometry variance in these patients. And even more important, no in vitro experiments for validation of a scoliotic model are available. The results may be somewhat different for patients and also for other spinal regions. As the role of, for example, the facet joint differs between the lumbar and the thoracic spine, the effect of the variance in the facet angle will also differ between the lumbar and thoracic spine. Furthermore, the effects are also depending on the magnitude of the load. We analysed loads from 0 to $10 \mathrm{Nm}$, which is a little more than the advised load for testing lumbar implants in vitro ${ }^{26}$. When higher loads are of interest, a larger effect for example of posterior ligaments in flexion or facet joints in axial rotation is expected, but of course care should be taken when extrapolating the results.

Thirdly, simplifications are made in all models and in ours as well. The most important simplification is the rigid body assumption for the vertebrae. This rigid body assumption causes a slight underestimation of the effects of vertebral height and of the lengths of the spinous and transverse processus. The rigid body assumption for the effect of the vertebral height, spinous process and transverse processes does, however, appears valid, because the main effect on the stiffness of the changes of these dimensions, are the changing moment arms for the ligament forces and not the increasing deformity of the vertebrae itself. This is also supported by a previous study $^{10}$, in which negligible effects on the stiffness for the vertebral height or the length of the spinous process of deformable vertebrae were found. It should however be noted, that in this study the height was varied with $5 \%$, while the natural variance in vertebral height is about $10 \%$. For all disc materials a linear stress-strain relation is assumed. Non-linear elastic behaviour is thus excluded, but also visco- and poroelastic behaviour is neglected. The latter are mainly important when loading is applied with high velocities, while the non-linear behaviour is especially important when a large range of loading is analysed. Furthermore, the facet joints are modelled as planes, rather than 3-dimensional contours. These assumptions were verified by validation of the model against in vitro results: load-displacement curves for the intact segment and various dissection stages, the instantaneous centre of rotation of the motion segment, and the load in the facet joints are compared with experimental data and proved the assumptions acceptable.

Lastly and most important, the interpersonal variance in spinal stiffness is dependent on the geometrical interpersonal variance as well as on the interpersonal variance in material properties of the various tissues. As there is no data on the variance in tissue mechanical properties, this study only analysed the effects of geometrical variance. We do, however, expect that the effect of interpersonal variance in mechanical properties is also large. Little and Adam ${ }^{27}$ have analysed the effects of soft tissue properties on the simulated outcome of a fulcrum bending test. A $40 \%$ change in the mechanical properties of the annulus fibres or of the ligaments 
changed the reduction of the Cobb angel in a fulcrum bending test with less than $1.2 \%$. These results in combination with the results from our current study indicate that, at least for scoliosis, the sensitivity for individual difference in tissue properties are much smaller than the sensitivity for differences in geometry. Unfortunately, it remains unclear what the actual physiological range in mechanical properties is.

In addition to obtain more personalized mechanical models of the spine, a currently often used method involves the correction of the stiffness based on side-bending radiographs ${ }^{7,28}$ Although this method is currently troubled by the fact that the applied forces to the spine are unknown, this could be improved by measuring or estimating these forces ${ }^{29}$. This method could then be used as a first estimate of a patient's spinal stiffness. If, however, more precise information is needed, this method is flawed. The estimated spinal stiffness is actually a result of the combined effects of patient-specific material properties and geometrical properties. A (linear) correction of the overall stiffness of the motion segment based on a single in vivo bending test is not appropriate to capture the non-linear geometrical effects that occur due to large deformities (common in spinal deformities and their correction). If the model distinguishes between the material and geometrical properties, results from sidebending radiographs can be used to personalize the material properties, after patientspecific geometry is already implemented. This makes extrapolation to other loading cases valid and improves the prediction of surgical outcome by patient-specific models.

Although no clinical recommendations can be based on this study, some major conclusions to improve and optimize patient-specific modelling are presented. Our results show that patient-specific spinal stiffness can be best predicted by the determination of the patient-specific disc height. The patient's endplate depth and endplate width, lordosis angle, facet joint orientation, and the cross-sectional area of the capsular ligament and supraspinous ligament are also of great influence on patient-specific stiffness, and are therefore important in patient-specific modelling. Other geometrical parameters can be ignored as these do not cause much of the interpersonal variance in spinal stiffness. 


\section{Key points}

Interpersonal variation of the disc height has a very large influence on spinal stiffness. The natural occurring range of disc height resulted in spinal stiffness ranging from $75 \%$ to $230 \%$ of the stiffness of an average spine. Accurate determining of disc height is therefore essential in patient-specific modelling.

Interpersonal variation in endplate depth and width, lordosis angle, facet joint angle and in the cross-sectional area of the capsular and supraspinous ligament had an influence of more than $5 \%$ on spinal stiffness.

Interpersonal variation in vertebral height, spinous process length, transverse process length, nucleus size, facet area, and the cross-sectional areas of most ligaments had little influence $(<5 \%)$ on the stiffness. For these parameters the use of average values appears acceptable, even for patient-specific models.

\section{Acknowledgement}

This study was financially supported by the Dutch Technology Foundation, STW, applied science division of NWO and the Technology Program of the Ministry of Economic Affairs.

We also thank ESI Group for the use of their software (PAM-CRASH). 


\section{References}

[1] B.K. Potter, M.K. Rosner, R.A. Lehman, D.W. Polly, T.M.M. Schroeder, T.R. Kuklo, 'Reliability of end, neutral, and stable vertebrae identification in adolescent idiopathic scoliosis'. Spine Vol. 30, pp. 1658-1663, 2005.

[2] J.A. Clamp, J.R. Andrews, M.P. Grevitt, 'A study of the radiologic predictors of curve flexibility in adolescent idiopathic scoliosis'. Journal of Spinal Disorders \& Techniques Vol. 21, pp. 213-215, 2008.

[3] D.W. Polly, P.F. Sturm, 'Traction versus supine side bending: which technique best determines curve flexibility? '. Spine Vol. 23, pp. 804-808, 1998.

[4] S.J. Klepps, L.G. Lenke, K.H. Bridwell, G.S. Bassett, J. Whorton, 'Prospective comparison of flexibility radiographs in adolescent idiopathic scoliosis'. Spine Vol. 26, pp. E74-E79, 2001.

[5] A. Hamzaoglu, U. Talu, M. Tezer, C. Mirzanl, U. Domanic, S.B. Goksan, 'Assessment of curve flexibility in adolescent Idiopathic scoliosis'. Spine Vol. 30, pp. 1637-1642, 2005.

[6] K.M.C. Cheung, K.D.K. Luk, 'Prediction of correction of scoliosis with use of the fulcrum bending radiograph'. J Bone Joint Surg Vol. 79-A, pp. 1144-1150, 1997.

[7] Y. Petit, C. Aubin, H. Labelle, 'Patient-specific mechanical properties of a flexible multi-body model of the scoliotic spine'. Medical and Biological Engineering and Computing Vol. 42, pp. 5560, 2004.

[8] C.E. Aubin, H. Labelle, C. Chevrefils, G. Desroches, J. Clin, A. Boivin, 'Preoperative planning simulator for spinal deformity surgeries'. Spine Vol. 33, pp. 2143-2152, 2008.

[9] B.J. Davis, A. Gadgil, J. Trivedi, E.-N.B. Ahmed, 'Traction radiography performed under general anesthetic: a new technique for assessing idiopathic scoliosis curves'. Spine Vol. 29, pp. 2466-2470, 2004.

[10] S. Robin, W. Skalli, F. Lavaste, 'Influence of geometrical factors on the behaviour of lumbar spine segmetns: a finite element analysis.'. European Spine J Vol. 3, pp. 84-90., 1994.

[11] R.N. Natarajan, G.B.J. Andersson, 'The influence of lumbar disc height and cross-sectional area on the mechanical response of the disc to physiologic loading.'. Spine Vol. 24, pp. 10031009, 1999.

[12] Y.M. Lu, W.C. Hutton, V.M. Gharpuray, 'Can variations in intervertebral disc height affect the mechanical function of the disc?'. Spine Vol. 21, pp. 2208-2216, 1996.

[13] G.J.M. Meijer, J. Homminga, E.E.G. Hekman, A.G. Veldhuizen, G.J. Verkerke, 'The effect of three-dimensional geometrical changes during adolescent growth on the biomechanics of a spinal motion segment'. J Biomech Vol. 43, pp. 1590-1597, 2010.

[14] M.M. Panjabi, V. Goel, T. Oxland, K. Takata, J. Duranceau, M. Krag, M. Price, 'Human lumbar vertebrae. Quantitative three-dimensional anatomy.'. Spine Vol. 17, pp. 299-306., 1992.

[15] M.F. Eijkelkamp On the development of an artificial intervertebral disc. PhD thesis, Rijksuniversiteit Groningen/ 2002.

[16] Y. Masharawi, B. Rothschild, G. Dar, S. Peleg, D. Robinson, E. Been, I. Hershkovitz, 'Facet orientation in the thorocolumbar spine. Three-dimensional anatomic and biomechanical analysis'. Spine Vol. 29, pp. 1755-1763., 2004.

[17] A.P. Dooris, V.K. Goel, N.M. Grosland, L.G. Gilbertson, D.G. Wilder, 'Load-sharing between anterior and posterior elements in a lumbar motion segment implanted with an artificial disc'. Spine Vol. 26, pp. E122-E129, 2001.

[18] F. Lavaste, W. Skalli, S. Robin, R. Roy-Camille, C. Mazel, 'Three-dimensional geometrical and mechanical modelling of the lumbar spine'. J Biomech Vol. 25, pp. 1153-1164., 1992.

[19] V.K. Goel, B.T. Monroe, L.G. Gilbertson, P. Brinckman, 'Interlaminar shear stresses and laminae separation in a disc. Finite element analysis of the L3-L4 motion segment subjected to axial compressive loads'. Spine Vol. 20, pp. 689-698., 1995.

[20] J. Chazal, A. Tanguy, M. Bourges, G. Gaurel, G. Escande, M. Guillot, G. Vanneuville, 'Biomechanical properties of spinal ligaments and a histological study of the supraspinal ligament in traction'. J Biomech Vol. 18, pp. 167-176., 1985.

[21] J.S. Little, P.S. Khalsa, 'Material properties of the human lumbar facet joint capsule'. J Biomech Eng Vol. 127, pp. 15-24, 2005. 
[22] A.A. White, M.M. Panjabi (1978) Clinical Biomechanics of the Spine. 1st edn. Lippincott Philadelphia, USA

[23] P. Neumann, T.S. Keller, L. Ekstrom, L. Perry, T.H. Hansson, D.M. Spengler, 'Mechanical properties of the human lumbar anterior longitudinal ligament'. J Biomech Vol. 25, pp. 11851194., 1992.

[24] F.A. Pintar, N. Yoganandan, T. Myers, A. Elhagediab, A. Sances, 'Biomechanical properties of human lumbar spine ligaments'. J Biomech Vol. 25, pp. 1351-1356, 1992.

[25] N. Yoganandan, S. Kumaresan, F.A. Pintar, 'Geometric and mechanical properties of human cervical spine ligaments'. J Biomech Eng Vol. 122, pp. 623-629, 2000.

[26] H.J. Wilke, K. Wenger, L. Claes, 'Testing criteria for spinal implants: recommendations for the standardization of in vitro stability testing of spinal implants'. European Spine Journal Vol. 7, pp. 148-154., 1998.

[27] J.P. Little, C.J. Adam, 'The effect of soft tissue properties on spinal flexibility in scoliosis: biomechanical simulation of fulcrum bending'. Spine Vol. 34, pp. E76-E82, 2009.

[28] Y. Lafon, V. Lafage, J.-P. Steib, J. Dubousset, W. Skalli, 'In vivo distribution of spinal intervertebral stiffness based on clinical flexibility tests'. Spine Vol. 35, pp. 186-193, 2010.

[29] M.-E. Lamarre, S. Parent, H. Labelle, C.-E. Aubin, J. Joncas, A. Cabral, Y. Petit, 'Assessment of spinal flexibility in adolescent idiopathic scoliosis: suspension versus sidebending radiography.'. Spine Vol. 34, pp. 591-597, 2009. 



\section{Chapter 5}

Influence of costovertebral joints on the stiffness of the spine

G.J.M. Meijer, J. Homminga, G.J. Verkerke

Submitted for publication 


\begin{abstract}
Study design

A validated finite element model is used to analyse the role of the posterior part of the ribs, including the costovertebral joints and ligaments and intercostal muscles, in the stiffness of the spine.

\section{Objective}

The objective of this study is to determine whether thoracic spinal segments including the posterior part of the ribs and attaching structures are representative for the mechanical behaviour of the isolated spine.

\title{
Summary of background Data
}

Since controlled removal is time consuming and thus influences the quality of the specimen, in vitro studies leave the posterior part of the ribs and attaching structures attached. Hence the role of these structures is unknown.

\section{Methods}

We created and validated models for three thoracic regions (T1-T4, T5-T8, and T9T12). These sections were modelled once with and once without the ribs and attaching structures. The mechanical behaviour of all models was then analysed for pure moments in flexion, extension, lateral bending and axial rotation.

\section{Results}

The mechanical behaviour for the multi-segment models with and without the ribs is comparable for flexion, extension and axial rotation, but not for lateral bending. In lateral bending, the posterior ribs and attaching structures increase the stiffness up to $40 \%$. By removal of just the intercostal muscles this increase in stiffness was reduced to less than $1 \%$.

\section{Conclusion}

Experimental results of spine including posterior part of the ribs and related structures can be used to describe the biomechanics of the isolated spine in flexion, extension and axial rotation, but not for lateral bending. To improve the in vitro test set up to represent the biomechanics of the isolated spine in lateral bending, the intercostal muscles can be dissected. 


\subsection{Introduction}

The function of the human spine requires it to be flexible, to allow trunk movement, and to be stiff, to provide stability to that same trunk. These functions are accomplished by a complex structure that uses multiple joints per motion segment as well as a multitude of ligaments and muscles. The thoracic spine is likely the most complex part of the spine; it not only has an intervertebral disc and facet joints, but it also has costovertebral joints between the ribs and vertebrae, costovertebral ligaments connecting the ribs to the vertebrae and intercostal muscles connecting the adjacent ribs.

In vitro mechanical tests have provided valuable information on the functioning of the thoracic spine and on the functional roles of the different parts. Such in vitro tests in the thoracic spine have been performed on several levels, from single motion segments $^{1,2}$, multi motion-segments ${ }^{3}$ to complete thoracic spines ${ }^{4}$. Since the costovertebral ligaments are anatomically intertwined with the spinal ligaments and the intervertebral disc, their removal is time-consuming and potentially harmful to the quality and biomechanical behaviour of the specimen ${ }^{5}$. For this reason, in vitro tests leave a part of the ribs, intercostal muscles, costovertebral joints and ligaments attached (these combined structures will be referred to as "posterior part of the ribs"). As a result, the mechanical effects of the posterior part of the ribs are unknown. Strictly speaking, the mechanical behaviour of the isolated thoracic spine is also unknown; to the best of our knowledge, only thoracic spines including the posterior part of the ribs have been studied.

The posterior part of the ribs can be expected to have an influence in lateral bending and, to a lesser extend in flexion-extension and axial rotation, but the magnitude of this influence is unknown.

In this study we set out to study this mechanical role of the posterior part of the ribs on the biomechanics of the spine, and determine the contribution of the (1) intercostal muscles, (2) costovertebral joint and radiate ligaments, (3) costotransverse joint and costotransverse ligaments. For this we created and used a finite element model (FE-model) of the thoracic spine, including the posterior $3 \mathrm{~cm}$ of the ribs, intercostal muscles, costovertebral joints and ligaments. By adding or eliminating the relevant structures from this FE-model, we were able to study the effects of the individual components on the mechanical behaviour of the thoracic spine. 


\subsection{Materials and Methods}

\subsubsection{Description of FE-model}

For this study we developed three FE-models of spinal segments (T1-T4, T5-T8 and T9-T12, figure 1). These models were constructed in a similar way as our previously published lumbar model ${ }^{6,7}$, which will be briefly summarized hereafter. The interaction between the thoracic spine and ribs will be described in more detail.

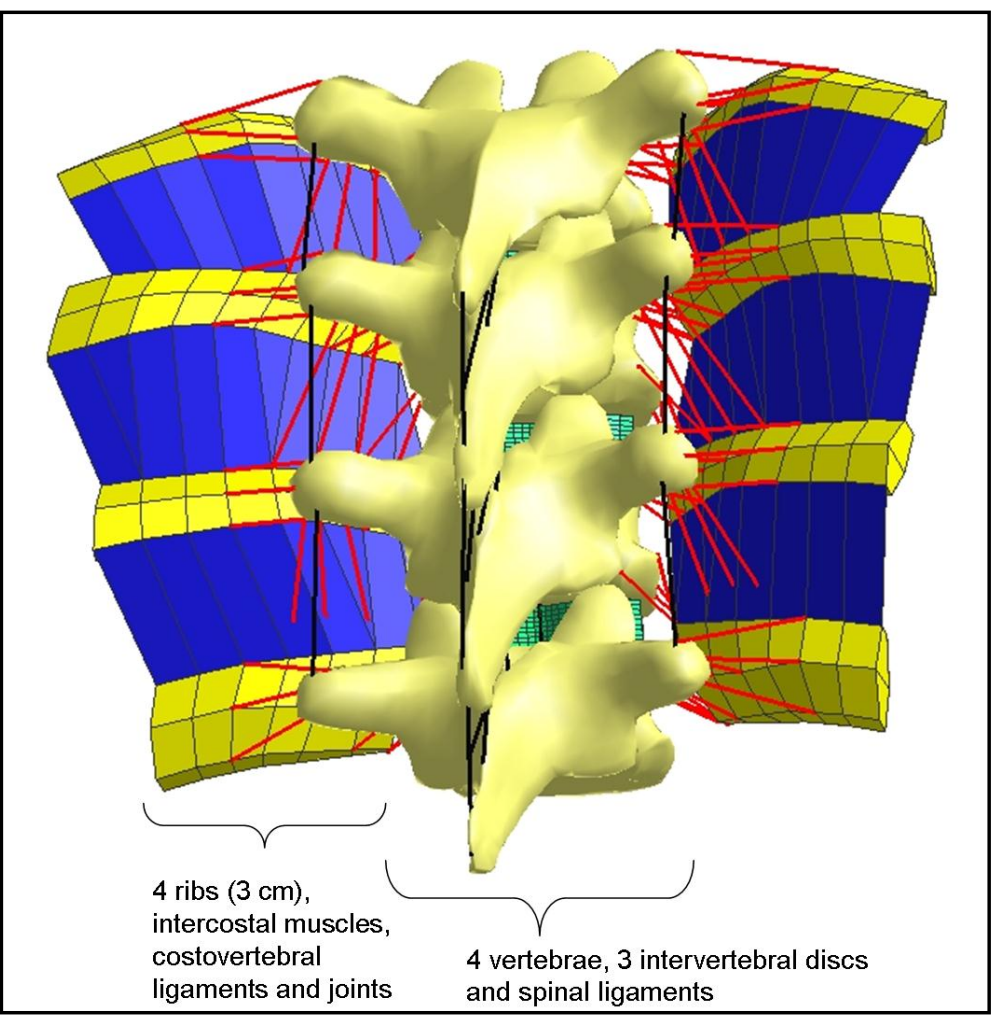

Figure 1: Finite Element model of T5-T8 including the vertebrae (modelled as rigid bodies), discs, spinal ligaments, posterior $3 \mathrm{~cm}$ of the ribs and intercostal muscles, costovertebral contacts and ligaments.

Each model spanned four vertebrae and contained vertebrae, intervertebral discs (with annulus and nucleus regions), facet joints, spinal ligaments, costovertebral joints and ligaments, posterior part of the ribs (on average $3 \mathrm{~cm}$ ) and intercostal muscles. Geometrical data for the spine was taken from literature and was specific for each vertebral level $^{8-10}$, and geometry of the ribcage was based on both anatomical literature ${ }^{11,12}$ and previous modelling studies ${ }^{13}$. The material properties were based on literature ${ }^{14-18}$ (table 1). 


\begin{tabular}{|c|c|c|}
\hline Structure & Material properties & Ref. \\
\hline \multirow{6}{*}{$\begin{array}{r}\text { Nucleus } \\
\text { Annulus matrix } \\
\text { Annulus fibres } \\
\text { ALL }\end{array}$} & $\mathrm{E}=1 \mathrm{MPa}, v=0.495$ & 14 \\
\hline & $\mathrm{E}=2 \mathrm{MPa}, v=0.45$ & 15 \\
\hline & $\mathrm{E}=450 \mathrm{MPa}$, volume fraction $=16 \%$ & 16 \\
\hline & $\mathrm{k}_{1}=52 \mathrm{~N} / \mathrm{mm}(\varepsilon<12 \%)$ & 17 \\
\hline & $\mathrm{k}_{2}=129 \mathrm{~N} / \mathrm{mm}(12 \%<\varepsilon<45 \%)$ & \\
\hline & $\mathrm{k}_{3}=65 \mathrm{~N} / \mathrm{mm}(\varepsilon>45 \%)$ & \\
\hline \multirow[t]{3}{*}{ PLL } & $\mathrm{k}_{1}=50 \mathrm{~N} / \mathrm{mm}(\varepsilon<9 \%)$ & 17 \\
\hline & $\mathrm{k}_{2}=118 \mathrm{~N} / \mathrm{mm}(9 \%<\varepsilon<34 \%)$ & \\
\hline & $\mathrm{k}_{3}=38 \mathrm{~N} / \mathrm{mm}(\varepsilon>34 \%)$ & \\
\hline \multirow[t]{3}{*}{$\mathbf{L F}$} & $\mathrm{k}_{1}=65 \mathrm{~N} / \mathrm{mm}(\varepsilon<5 \%)$ & 16 \\
\hline & $\mathrm{k}_{2}=85 \mathrm{~N} / \mathrm{mm}(5 \%<\varepsilon<50 \%)$ & \\
\hline & $\mathrm{k}_{3}=26 \mathrm{~N} / \mathrm{mm}(\varepsilon>50 \%)$ & \\
\hline \multirow[t]{3}{*}{ ISL } & $\mathrm{k}_{1}=24 \mathrm{~N} / \mathrm{mm}(\varepsilon<12 \%)$ & 17 \\
\hline & $\mathrm{k}_{2}=53 \mathrm{~N} / \mathrm{mm}(12 \%<\varepsilon<30 \%)$ & \\
\hline & $\mathrm{k}_{3}=25 \mathrm{~N} / \mathrm{mm}(\varepsilon>30 \%)$ & \\
\hline \multirow[t]{3}{*}{ SSL } & $\mathrm{k}_{1}=18 \mathrm{~N} / \mathrm{mm}(\varepsilon<12 \%)$ & 17 \\
\hline & $\mathrm{k}_{2}=40 \mathrm{~N} / \mathrm{mm}(12 \%<\varepsilon<30 \%)$ & \\
\hline & $\mathrm{k}_{3}=19 \mathrm{~N} / \mathrm{mm}(\varepsilon>30 \%)$ & \\
\hline \multirow[t]{3}{*}{ ITL } & $\mathrm{k}_{1}=28 \mathrm{~N} / \mathrm{mm}(\varepsilon<9 \%)$ & 17 \\
\hline & $\mathrm{k}_{2}=137 \mathrm{~N} / \mathrm{mm}(9 \%<\varepsilon<15 \%)$ & \\
\hline & $\mathrm{k}_{3}=46 \mathrm{~N} / \mathrm{mm}(\varepsilon>15 \%)$ & \\
\hline \multirow[t]{3}{*}{$\mathbf{C L}$} & $\mathrm{k}_{1}=18 \mathrm{~N} / \mathrm{mm}(\varepsilon<100 \%)$ & 18 \\
\hline & $\mathrm{k}_{2}=45 \mathrm{~N} / \mathrm{mm}(100 \%<\varepsilon<200 \%)$ & \\
\hline & $\mathrm{k}_{3}=15 \mathrm{~N} / \mathrm{mm}(\varepsilon>200 \%)$ & \\
\hline $\begin{array}{r}\text { Costovertebral and } \\
\text { costotransverse ligaments }\end{array}$ & See table 2 & - \\
\hline Ribs & $\mathrm{E}=10.7 \mathrm{GPa}, v=0.3$ & 19 \\
\hline Intercostal muscles matrix & $\mathrm{E}=5 \mathrm{MPa}, \nu=0.4$ & - \\
\hline Intercostal muscles fibres & $\mathrm{E}=25 \mathrm{MPa}, v=0.3$ & - \\
\hline $\begin{array}{l}\text { Facet surfaces and } \\
\text { costovertebral contacts }\end{array}$ & Stiffness increases with compression depth & - \\
\hline Vertebrae & Rigid bodies & - \\
\hline
\end{tabular}

Table 1: Overview of material properties

As the spine's flexibility is dominated by the soft tissues of the spine, the vertebrae are modelled as rigid bodies, while discs, ligaments and muscles are represented by a deformable mesh. The intervertebral disc consisted of an incompressible nucleus and an annulus which contains a base-material reinforced by fibres in a physiological crisscross pattern. All spinal ligaments (ALL, PLL, LF, ISL, SSL, ITL and CL, see table 3 for an explanation of the abbreviations) were represented by non-linear tension-only elements. Mechanical properties of the costovertebral ligaments have never been measured, as noted in previous modelling studies. For this study, we assumed the Young's modulus of these ligaments to increase from that of elastin $(E=0.6 \mathrm{MPa})^{20}$ to that of collagen $\left(E=1 \mathrm{GPa}^{20}\right.$, table 2$)$. 


\begin{tabular}{|cl|}
\hline $\begin{array}{c}\text { Strain } \\
{[\%]}\end{array}$ & $\begin{array}{c}\text { E-modulus } \\
{[\mathrm{MPa}]}\end{array}$ \\
\hline $0.0-0.001$ & 0.6 \\
$0.001-0.001$ & 1.2 \\
$0.01-0.05$ & 2.5 \\
$0.05-0.1$ & 50 \\
$0.1-0.5$ & 100 \\
$0.5-1.0$ & 200 \\
$>1.0$ & 1000 \\
\hline
\end{tabular}

Table 2: Mechanical properties of costovertebral and costotransverse ligaments

The low-strain behaviour of the costovertebral ligaments is thus comparable to that of the spinal ligaments. This is also the applicable region for this study, since the much stiffer high strain behaviour is not reached during the prescribed loading conditions. Cross-sectional areas for each of the ligaments are based on literature ${ }^{11,16,17}$ and are presented in table 3.

\begin{tabular}{|rcc|} 
Ligament & $\begin{array}{c}\text { Area } \\
{\left[\mathbf{m m}^{2}\right]}\end{array}$ & Ref. \\
\hline ALL & 31 & 17 \\
PLL & 18 & 17 \\
LF & 27 & 17 \\
ISL & 30 & 16 \\
SSL & 10 & 16 \\
ITL * & 2 & 17 \\
CL * & 30 & 16 \\
CVL * & 10 & 11 \\
\hline *) data per side & \\
\hline
\end{tabular}

Table 3: Cross-sectional area of ligaments

Abbreviations: $A L L=$ anterior longitudinal ligament, $P L L=$ posterior longitudinal ligament, $L F$ $=$ ligament flavum, $I S L=$ Interspinous ligament, $S S L=S$ upraspinous ligament, $I T L=$ intertransverse ligament, $C L=$ capsular ligament, $C V L=$ costovertebral and costotransverse ligaments.

For muscles, the only reported mechanical property is the maximum stress, which is reported to be between 0.4 and $0.65 \mathrm{MPa}$ for trunk muscles ${ }^{21}$; an average of 0.5 $\mathrm{MPa}$ is assumed for the intercostal muscles. To determine the E-modulus of the fibres, a strain of $0.5 \%$ is assumed in combination with $25 \%$ of the maximum stress, resulting in an E-modulus of $25 \mathrm{MPa}$ for the fibres. For the matrix, the strain is assumed to be $1 \%$ and the stress is set at $10 \%$ of the maximum level, resulting in an E-modulus of $5 \mathrm{MPa}$.

The facet joints, costovertebral and costotransverse joints were modelled as nonlinear penalty contacts. The mechanical properties of the ribs were based on experimental data for the posterior part of the ribs ${ }^{19}$. The intercostal muscles were modelled as a base-material reinforced by fibres to represent the orientation of the muscles. A different orientation for the internal and external part of the muscles is reported. The orientation of the fibres in the outer layer is 30 degrees with respect to the ribs, in the internal layer this orientation is 120 degrees $^{22}$. 


\subsubsection{Validation of costovertebral joints}

The mechanical behaviour of the costovertebral and costotransverse joints, ligaments and the posterior $3 \mathrm{~cm}$ of the ribs was compared to in vitro experiments ${ }^{23,24}$. The T1-T2, T5-T6, and T9-T10 motion segments including the respective rib (on average $3 \mathrm{~cm}$ ), and the related costovertebral contacts and ligaments were modelled and loaded as in the in vitro studies: the vertebrae were fully fixed and the ribs were loaded with pure moments increasing from 0 to $0.5 \mathrm{Nm}$. The motions of the rib relative to the vertebrae were compared to those measured in the in vitro studies. In the experimental studies, ROM (Range Of Motion) was defined as the range of motion between -0.1 and $0.1 \mathrm{Nm}$, except for torsion, where only the positive direction was tested $(0$ to $0.1 \mathrm{Nm}$ ). For comparison to the other loading directions, the range of motion in torsion between 0 and $0.1 \mathrm{Nm}$ was multiplied by two to represent the ROM between -0.1 and $+0.1 \mathrm{Nm}$. This was done for both the results of the in vitro experiments and the simulations.

\subsubsection{Validation of thoracic spinal segments}

The complete models (T1-T4, T5-T8, and T9-T12) of four vertebra, four ribs, costovertebral joints, ligaments and intercostal muscles are also validated against in vitro tests ${ }^{3}$. In these simulations, the lowermost vertebra is fully fixed and pure moments are applied to the uppermost vertebra. Moments ranging from 0 to $4 \mathrm{Nm}$ are applied in flexion, extension, lateral bending and axial rotation. Moments ranging from $0-4 \mathrm{Nm}$ are applied in flexion, extension, lateral bending and axial rotation. The rotation of the uppermost vertebra relative to the fixed lowermost vertebra (Range Of Motion, ROM) is measured and compared to those measured in the in vitro study.

\subsubsection{Mechanical effect of the posterior part of the ribs}

The three, validated models of spinal multi-segments (T1-T4, T5-T8 and T9-T12) are then used to analyse the mechanical effect of the posterior part of the ribs and related structures. The lowermost vertebra of each segment was fully fixed and the uppermost vertebra was loaded with pure moments (increasing from 0-4 Nm) in flexion, extension, lateral bending and axial rotation. For these simulations, the rotation of the uppermost vertebra relative to the fixed lowermost vertebra is determined and the load-displacement behaviour is compared for the isolated spine and for the spine including the posterior part of the ribs. Subsequently we analysed the individual effect of removal of (1) intercostal muscles, or (2) costovertebral joint and radiate ligaments, or (3) costotransverse joint and costotransverse ligaments by comparing the 3 dissected models to the intact model.

\subsection{Results}

\subsubsection{Validation of costovertebral joint}

The measured and simulated ROM under low moments $(-0.1$ to $+0.1 \mathrm{Nm})$ change dramatically with the loading direction (figure 2, left). 


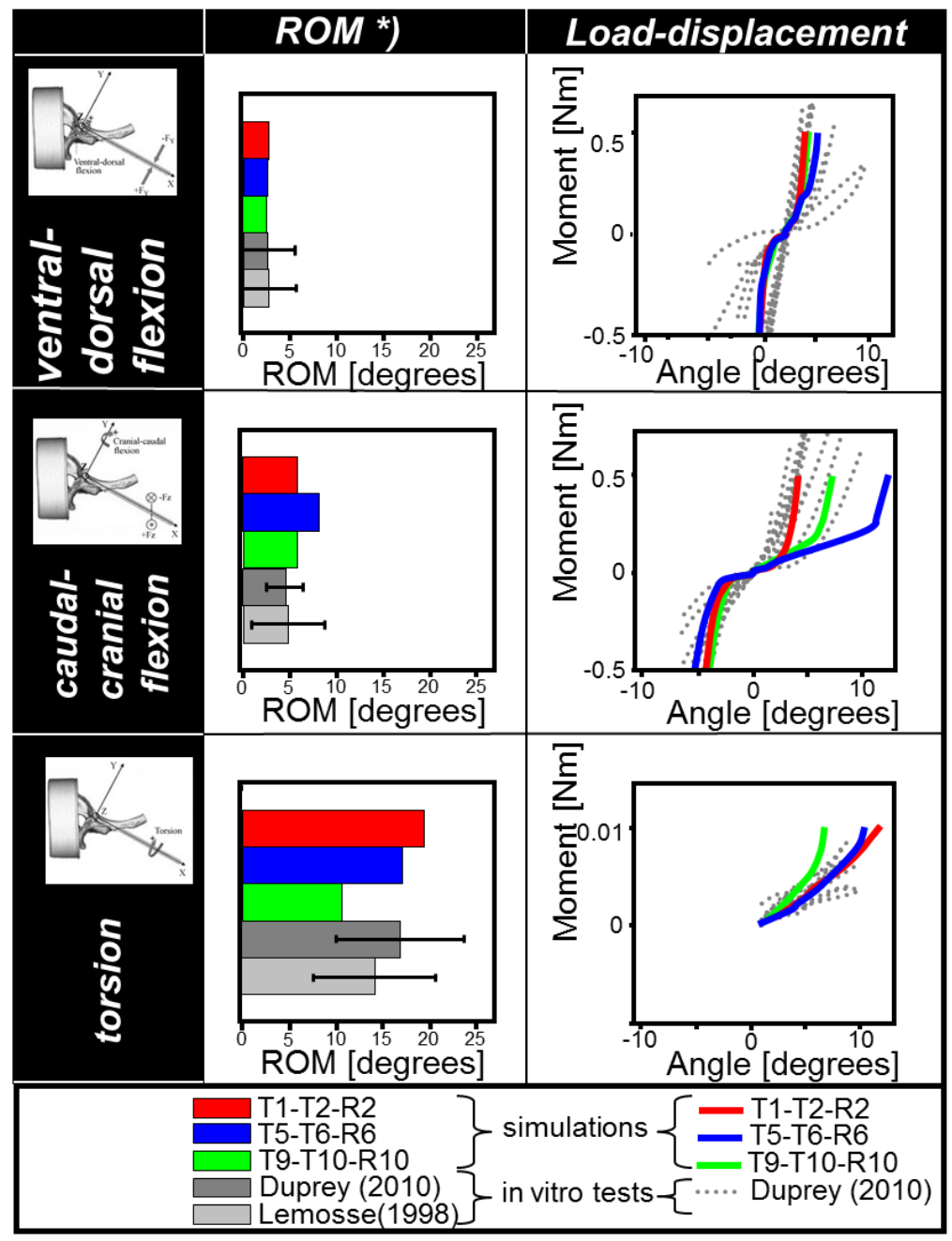

Figure 2: Load-displacement behaviour of the costovertebral joint: model versus experimental studies. Error bars show the standard deviation in the experimental data.

*) ROM was defined as the range of motion between -0.1 and $0.1 \mathrm{Nm}$.

Both the experimental and simulated results show that the costovertebral joints are far more flexible under rotational moments (torsion) than under caudal-cranial or ventral-dorsal bending. Also the rotational flexibility is higher for higher thoracic segments. Such an effect of the region was not observed for caudal-cranial and ventral-dorsal bending. The experiments of Lemosse et al. ${ }^{24}$ show that for all movement directions the mid-thoracic costovertebral joint is the most stiff. This is likely caused by the fact that the anterior-posterior and medial-lateral dimension are 
largest for this rib (complete ribs are tested in this experiment). Since the specimen in the experiment of Duprey et al. ${ }^{23}$ and in our models all include comparable lengths of ribs (average of $3 \mathrm{~cm}$ ), this effect is absent. All calculated ROM-values are comparable to the previously found experimental values ${ }^{24,23}$ (figure 2, left). The detailed load-displacement behaviour under larger moments is also comparable to previously found experimental behaviour ${ }^{23}$ (figure 2, right).

\subsubsection{Validation of thoracic spinal multi-segments}

The mechanical behaviour for the complete spinal multi-segment models (four vertebra, four ribs, costovertebral joints, ligaments and intercostal muscles) are compared to the results found during in vitro experiments ${ }^{3}$, by com paring the ROM for the middle disc (figure 3).

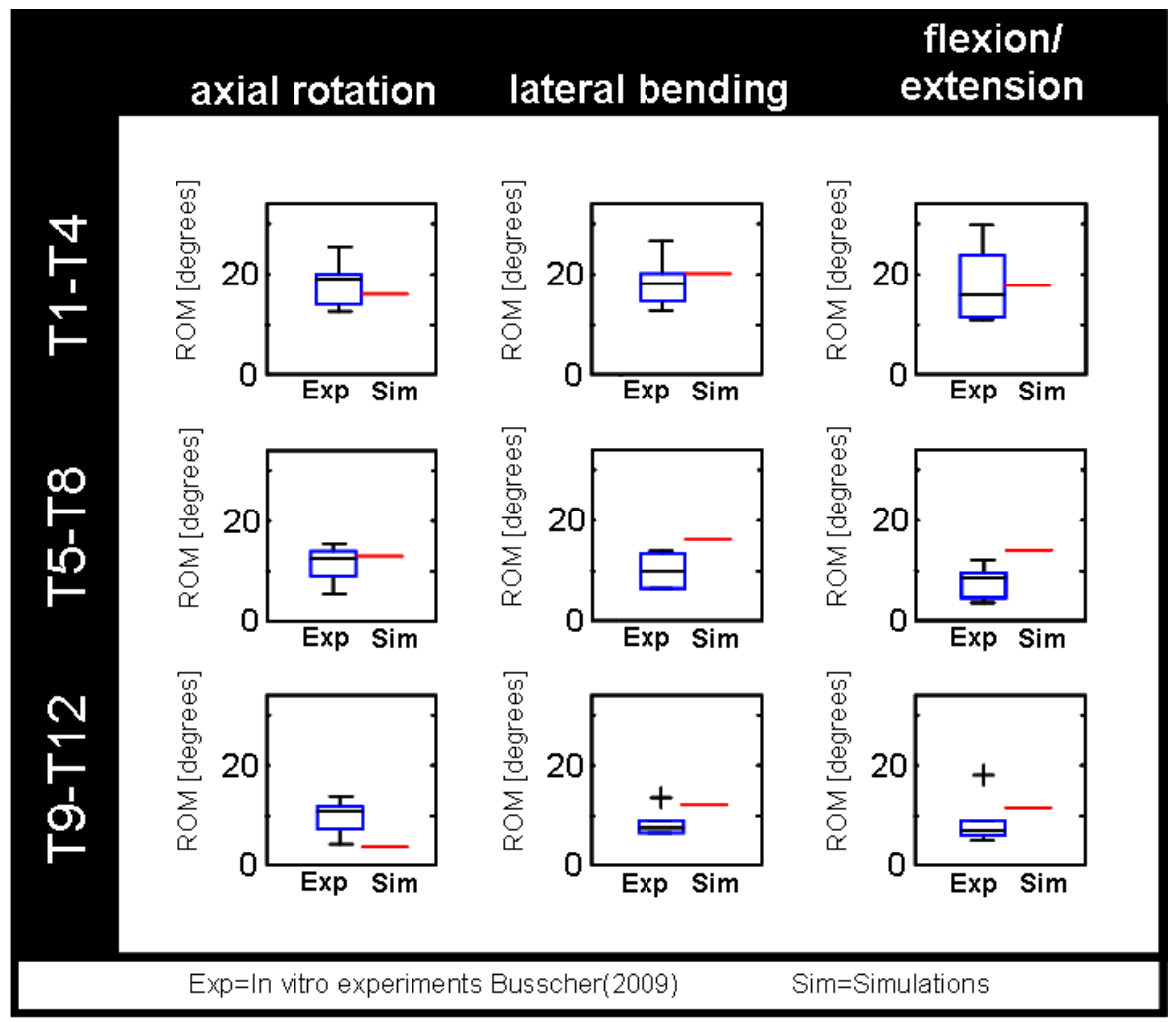

Figure 3: ROM (between $-4 \mathrm{Nm}$ and $+4 \mathrm{Nm}$ ) of the multi-segment model including ribs compared to in vitro experiments. Experimental data are presented as box plots, showing the average, the range and outliers of the data.

The results, of both the in vitro experiments and of our models, demonstrate a higher ROM in all directions for the upper-thoracic spine compared to the mid- and lower- 
thoracic spine. The mechanical results of the spinal models are within the found experimental range, except for the T5-T8 segments in flexion/extension and lateral bending. This will be further analysed in the discussion.

\subsubsection{Mechanical effect of the posterior part of the ribs}

Figure 4 displays the mechanical effect of the removal of the posterior part of the ribs on the biomechanics of the spine.

Surprisingly, the removal had virtually no effect on the mechanical behaviour of the spinal segments in axial torsion, flexion, and extension. Under lateral bending the added mechanical stiffness (averaged for the loading range) due to the posterior parts of the ribs was considerable: $16 \%$ for the upper thoracic region, $37 \%$ for the mid thoracic region, and $15 \%$ for the lower thoracic region.

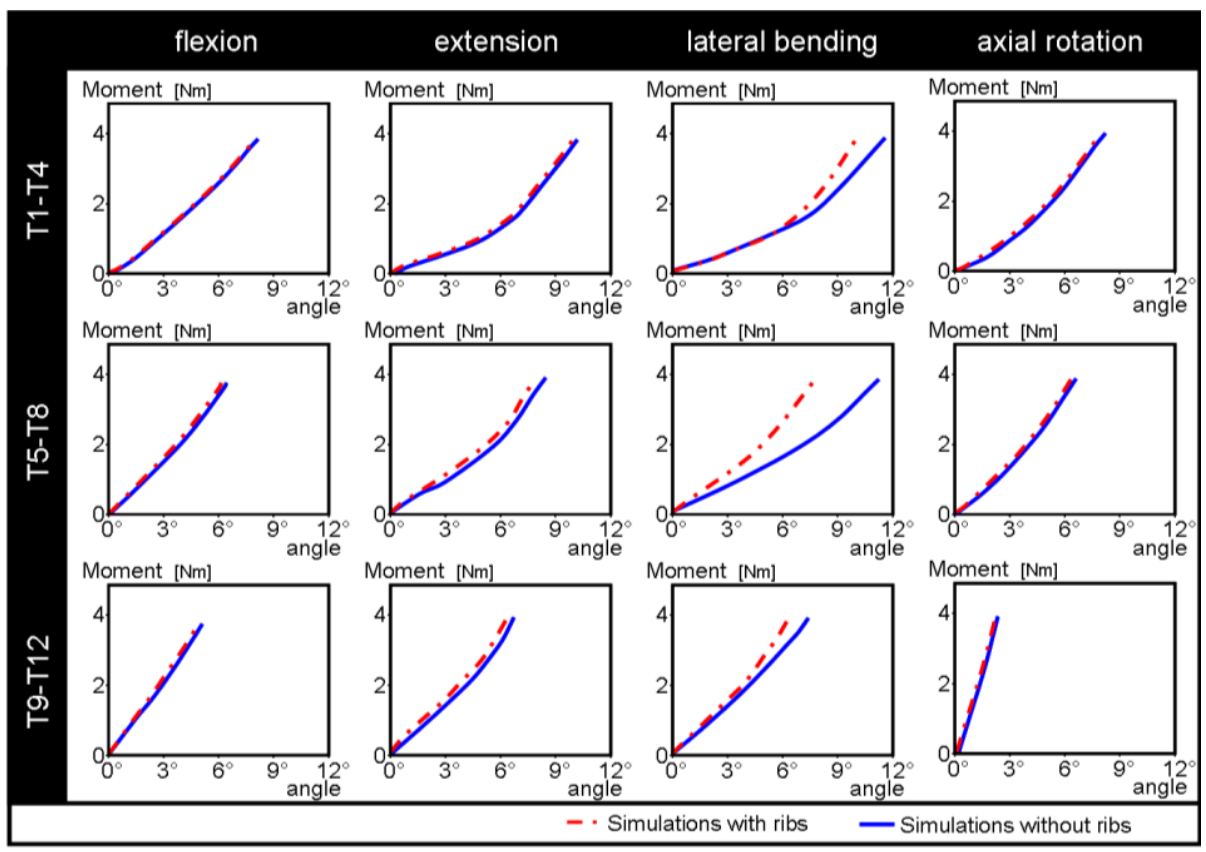

Figure 4: Influence of the remaining posterior part of the ribs on the load-displacement behaviour of the spinal multi-segments.

In our analysis into the relative influence (on the average stiffness) of the substructures we removed either the intercostal muscles, or the costovertebral joint and radiate ligaments, or the costotransverse joint and costotransverse ligaments (table 4). 


\begin{tabular}{|rcccc|}
$\begin{array}{r}\text { spinal } \\
\text { region }\end{array}$ & $\begin{array}{c}\text { intercostal } \\
\text { muscles }\end{array}$ & $\begin{array}{c}\text { costovertebral joints } \\
\text { and radiate ligaments }\end{array}$ & $\begin{array}{c}\text { costotransverse joints and } \\
\text { costotransverse ligaments }\end{array}$ & $\begin{array}{c}\text { total } \\
\text { posterior } \\
\text { rib parts }\end{array}$ \\
\hline T1-T4 & $95 \%$ & $79 \%$ & $85 \%$ & $100 \%$ \\
T5-T8 & $99 \%$ & $55 \%$ & $99 \%$ & $100 \%$ \\
T9-T12 & $98 \%$ & $88 \%$ & $79 \%$ & $100 \%$ \\
\hline
\end{tabular}

Table 4: Relative effect on the average stiffness in lateral bending of the removal of the various structures, as a percentage of the total removal of the posterior rib parts

For all regions, the largest effect was seen for removal of the intercostal muscles reducing the added stiffness due to the presence of the ribs by $95-99 \%$. The least effect was found for the removal of the costovertebral joint and ligaments (T1-T4 and T5-T8 segments), and for the removal of the costotransverse joints and radiate ligaments (T9-T12). The combined effect of the posterior part of the ribs is not simply the sum of these separate effects. All individual effects were larger than $55 \%$, while the total effect can naturally only be $100 \%$.

\subsection{Discussion}

The goal of this study was to study the influence of the posterior part of the ribs on the stiffness of the thoracic spine. For this purpose, both the behaviour of the modelled costovertebral joints and the total multi-segments are validated against in vitro experiments. The mechanical behaviour of the costovertebral joint fits well with the available experimental data ${ }^{24,23}$. The difference between the various movement directions and regions are captured well by the model. The multi-segment models were also able to capture the behaviour of the tested spines rather nicely. Again, the difference in regions and movement directions as found in the experiments ${ }^{3}$ was captured by the models. The mechanical results of the spinal models are within the found experimental range, except for the T5-T8 segments in flexion/extension and lateral bending. An important difference between the in vitro experiments and current models, are the age and the related degenerated state of the intervertebral disc. The in vitro experiments are carried out in aged spines (average age 72 years, mild degeneration is reported) while our models are based on data for healthy, younger subjects. It seems likely that degeneration affects especially the height of the disc, which again is reported to highly affect the stiffness of the spine ${ }^{7,25}$. Our models have a high ROM compared to the experimental data, which is expected when assuming that in the experiments the disc height is reduced due to degeneration, resulting in a higher stiffness. Overall, the models predict the mechanical behaviour of thoracic spine and the posterior part of the ribs sufficiently well.

Our results demonstrate that the removal of the posterior part of the ribs and related structures only considerably affects the mechanical behaviour in lateral bending. The mechanical behaviour of the spinal segments in flexion, extension and axial torsion is unaffected. Our simulations of the removal of the individual parts showed that the added stiffness in lateral bending is mainly caused by the intercostal muscles.

The effect of the various substructures of the posterior part of the ribs depends on the spinal region. Although all regions showed a large effect for the intercostal muscles, the relative effect of the costotransverse and costovertebral joint and 
ligaments was different for the various regions. The effect of the costotransverse joints and radiate ligaments was smaller in the low thoracic region, which is logical as these joints are absent for the T11 and T12 vertebrae, thus lowering their role for the entire T9-T12 segment. The relative effect of the costovertebral and costotransverse joints and ligaments can also be explained when analysing the orientation of the axis through the costovertebral and the costotransverse joint. For the low thoracic spine, this axis is parallel to the sagittal plane, for the high thoracic spine it is close to the frontal plane and for the mid thoracic region it is in between, at an angle of 45 degrees with the sagittal plane. Lateral bending of the spine will thus mainly result in a lateral movement of the ribs for the lower spine region, in an anterior displacement for the high spine region and in a both lateral and anterior displacement of the ribs for the mid thoracic region. This combined movement of the ribs in the mid thoracic region enforces extra axial rotation (coupled movements) to the spine, which results in the lesser role of the costovertebral joints in the mid thoracic region.

Some limitations apply to this study. Firstly, the exact material properties of costovertebral ligaments and intercostal muscles are unknown. The currently used values for the costovertebral ligaments are based on a transition from more elastinlike behaviour for low strains to a more collagen-like behaviour for high strains. For muscles, the only reported mechanical property is the maximum stress. To determine the E-modulus of the fibres, a strain in combination with a percentage of the maximum stress is assumed. These assumptions were validated by comparing various models to experimental data; the individual costovertebral rib-joint (figure 2), multi-segments (figure 3) but also the total ribcage (not presented in this study) show comparable behaviour to the experimental data. Though the used material behaviour seems thus realistic, actual measurements of the properties would be valuable.

Secondly, the current study focused on the behaviour of spinal sections, containing four vertebrae. For shorter spine sections, the effects of the posterior parts of the ribs and related structures can be expected to be smaller, while for longer sections the effects can be expected to be larger and could thus also be considerable in other movement directions. In our current study the top and bottom ribs are only connected on one side, while in a complete spine these ribs would be connected on both sides, which should result in a larger effect on the overall stiffness than what was determined in this study.

The results of the study implies that in vitro experiments including the posterior parts of the ribs and related structures, are very representative for the biomechanical behaviour of the isolated spine in flexion, extension and axial rotation. For lateral bending, however, the hitherto performed in vitro experiments, that do include the posterior parts of the ribs and related structures, are far less representative for the biomechanical behaviour of the isolated spine. The posterior ribs and attaching structures increase the stiffness up to $40 \%$, but removal of just the intercostal muscles reduces this increase in stiffness to less than $1 \%$. In in vitro studies this effect could thus easily be reduced by making a horizontal incision in between the ribs, dissecting the attaching muscles, before starting the in vitro experiments.

In conclusion, for shorter spine sections (< four vertebrae), the posterior parts of the ribs and related structures only affects the mechanical behaviour in lateral bending. This effect is mainly due to the intercostal muscles, although the costotransverse 
joint and ligaments and the costovertebral joint and radiate ligaments were almost equally important. The effects on longer spine sections remains to be determined, but is expected to be larger than the results reported in this study.

\section{Key points}

When the thoracic spine is used in in vitro tests, the posterior parts of the ribs, costovertebral contacts and ligaments are always left attached for practical reasons. The role of these structures has thus never been evaluated.

Costovertebral joints, ligaments and first $3 \mathrm{~cm}$ of the ribs add little to the stiffness of an isolated spine in flexion-extension and torsion. In lateral bending they increase the stiffness by $15-40 \%$.

Increase in lateral bending stiffness is mainly caused by the intercostal muscles. Removing these intercostal muscles (by a simple horizontal incision in between the ribs) reduced the increase in stiffness due to the presence of the ribs to less than $1 \%$.

\section{Acknowledgement}

This study was financially supported by the Dutch Technology Foundation, STW, applied science division of NWO and the Technology Program of the Ministry of Economic Affairs.

We also thank ESI Group for the use of their software (PAM-CRASH). 


\section{References}

[1] M.M. Panjabi, R.A. Brand, A.A. White, 'Mechanical properties of the human thoracic spine as shown by three-dimensional load-displacement curves'. J Bone Joint Surg Vol. 58-A, pp. 642652, 1976.

[2] M.M. Panjabi, J.N. Hausfeld, A.A. White, 'A biomechanical study of the ligamentous stability of the thoracic spine in man'. Acta Orthop Scand Vol. 52, pp. 315, 1981.

[3] I. Busscher, J.H. van Dieen, I. Kingma, A.J. van der Veen, G.J. Verkerke, A.G. Veldhuizen, 'Biomechanical characteristics of different regions of the human spine: an in vitro study on multilevel spinal segments'. Spine Vol. 34, pp. 2858-2864, 2009.

[4] R. Watkins, R. Watkins, L. Williams, S. Ahlbrand, R. Garcia, A. Karamanian, L. Sharp, C. Vo, T.P. Hedman, 'Stability provided by sternum and rib cage in the thoracic spine.'. Spine Vol. 30, pp. 1283-1286, 2005.

[5] H.J. Wilke, B. Jungkanz, K. Wenger, L.E. Claes, 'Spinal segment range of motion as a function of in vitro test conditions: effects of exposure period, accumulated cycles, angulardeformation rate and moisture condition.'. Anat Rec Vol. 251, pp. 15-19, 1998.

[6] G.J.M. Meijer, J. Homminga, E.E.G. Hekman, A.G. Veldhuizen, G.J. Verkerke, 'The effect of three-dimensional geometrical changes during adolescent growth on the biomechanics of a spinal motion segment'. J Biomech Vol. 43, pp. 1590-1597, 2010.

[7] G.J.M. Meijer, J. Homminga, A.G. Veldhuizen, G.J. Verkerke, 'Influence of interpersonal geometrical variation on spinal motion segment stiffness: Implications for patient-specific modeling'. Spine Vol. 36, pp. E929-E935 2011.

[8] M.M. Panjabi, K. Takata, V. Goel, D. Federico, T. Oxland, J. Duranceau, M. Krag, 'Thoracic human vertebrae. Quantitative three-dimensional anatomy.'. Spine Vol. 16, pp. 888-901., 1991.

[9] K. Chanchairujira, C.B. Chung, J.Y. Kim, O. Papakonstantinou, M.H. Lee, P. Clopton, D. Resnick, 'Intervertebral disk calcification of the spine in an elderly population: radiographic prevalence, location, and distribution and correlation with spinal degeneration'. Radiology Vol. 230, pp. 499-503, 2004.

[10] Y. Masharawi, B. Rothschild, G. Dar, S. Peleg, D. Robinson, E. Been, I. Hershkovitz, 'Facet orientation in the thorocolumbar spine. Three-dimensional anatomic and biomechanical analysis'. Spine Vol. 29, pp. 1755-1763., 2004.

[11] I.A. Kapandji, L.H. Honoré (1985) The physiology of the joints. Volume 3: The trunk and the vertebral column. 1st edn. Ferdinand Enke Verlag, Stuttgart, Germany

[12] Y.P. Charles, A. Diméglio, M. Marcoul, J.-F. Bourgin, A. Marcoul, M.-C. Bozonnat, 'Influence of idiopathic scoliosis on three-dimensional thoracic growth'. Spine Vol. 33, pp. 12091218., 2008.

[13] S. Na, S. Sah, D. Hwang, H.Y. Choi, I. Lee, J. Lee, 'Numerical simulation of out-of-position driver side airbag deployment with small female model'. JSAE Annual Congress Vol. 8, pp. 5-8, 2003.

[14] A.P. Dooris, V.K. Goel, N.M. Grosland, L.G. Gilbertson, D.G. Wilder, 'Load-sharing between anterior and posterior elements in a lumbar motion segment implanted with an artificial disc'. Spine Vol. 26, pp. E122-E129, 2001.

[15] F. Lavaste, W. Skalli, S. Robin, R. Roy-Camille, C. Mazel, 'Three-dimensional geometrical and mechanical modelling of the lumbar spine'. J Biomech Vol. 25, pp. 1153-1164., 1992.

[16] V.K. Goel, B.T. Monroe, L.G. Gilbertson, P. Brinckman, 'Interlaminar shear stresses and laminae separation in a disc. Finite element analysis of the L3-L4 motion segment subjected to axial compressive loads'. Spine Vol. 20, pp. 689-698., 1995.

[17] J. Chazal, A. Tanguy, M. Bourges, G. Gaurel, G. Escande, M. Guillot, G. Vanneuville, 'Biomechanical properties of spinal ligaments and a histological study of the supraspinal ligament in traction'. J Biomech Vol. 18, pp. 167-176., 1985.

[18] J.S. Little, P.S. Khalsa, 'Material properties of the human lumbar facet joint capsule'. J Biomech Eng Vol. 127, pp. 15-24, 2005.

[19] J.D. Stitzel The role of local material properties in modeling fracture tolerance of the human thorax. PhD thesis, Viriginia polytechnic institute and state university/ Blacksburg, Virginia, 2003. 
[20] Y.C. Fung (1993) Biomechanics : mechanical properties of living tissues. 2nd edn. Springer [21] M.A. Nussbaum, B.J. Martin, D.B. Chaffin, 'A neural network model for simulation of torso muscle coordination'. J Biomech Vol. 30, pp. 251-258, 1997.

[22] T.A. Wilson, A. Legrand, P.-A. Gevenois, A. De Troyer, 'Respiratory effects of the external and internal intercostal muscles in humans'. The Journal of Physiology Vol. 530, pp. 319-330, 2001.

[23] S. Duprey, D. Subit, H. Guillemot, R.W. Kent, 'Biomechanical properties of the costovertebral joint'. Med Eng Phys Vol. 32, pp. 222-227, 2010.

[24] D. Lemosse, O. Le Rue, A. Diop, W. Skalli, P. Marec, F. Lavaste, 'Characterization of the mechanical behaviour parameters of the costo-vertebral joint'. European Spine Journal Vol. 7 , pp. 16-23, 1998.

[25] R.N. Natarajan, G.B.J. Andersson, 'The influence of lumbar disc height and cross-sectional area on the mechanical response of the disc to physiologic loading.'. Spine Vol. 24, pp. 10031009, 1999. 



\section{Chapter 6}

Mechanical role of the spine, ribcage and intraabdominal pressure in the behaviour of the trunk

G.J.M. Meijer, J. Homminga, G.J. Verkerke

Submitted for publication 


\begin{abstract}
The biomechanics of the human trunk is very complex, and the role of some of its parts is unclear. In particular the exact mechanism and quantitative contribution of the intra-abdominal pressure (IAP) is unclear, although it is known to have a large role in the stiffness and stability of the trunk. In this study, we studied the mechanical role of the spine, ribcage and intra-abdominal pressure in the behaviour of the trunk, using a very complete finite element model of the human trunk.

Our results show that both the ribcage and intra-abdominal pressure have a large effect on the stiffness of the trunk (19-25\% and $50-69 \%$ respectively). We also found that the ribcage mainly influences the mid-thoracic region (T4-T10), while the IAP mainly influences the low-thoracic and lumbar regions (T6-L5). These effects are seen for all movement directions (flexion, extension, lateral bending and axial rotation).
\end{abstract}




\subsection{Introduction}

The biomechanics of the human trunk is very complex, and the role of some of its parts remains unclear. In particular the exact mechanism and quantitative contribution of the intra-abdominal pressure (IAP) is unclear, although it is known to have a large role in the stiffness and stability of the trunk ${ }^{1-5}$. This lack of knowledge is caused by the practical limitations of in vitro and in vivo experiments and by simplifications in computational studies. Also, while the role of the ribcage in the mechanics of the spine has been determined ${ }^{6,7}$, the role in the behaviour of the total trunk remains unclear.

Numerical models can help to improve our insight in the mechanics of the trunk. The current literature, however, contains no studies analysing the mechanics of the trunk that includes the spine, ribcage, intra-abdominal pressure and muscles.

In this study, we studied the mechanical role of the spine, ribcage and intraabdominal pressure in the behaviour of the trunk, using a very complete finite element model of the human trunk.

\subsection{Methods}

\subsubsection{Finite element models}

Based on our previously developed models of a lumbar motion segment ${ }^{8,9}$ and thoracic multi-segments ${ }^{10}$, we developed four models of an average adolescent trunk; thoracolumbar spine, spine including $3 \mathrm{~cm}$ ribs, spine including total ribcage, and spine including ribcage and IAP ( figure 1).

For illustration purposes, the sacrum and pelvis are included in the models, but mechanically the $\mathrm{L} 5$, the pelvis and the sacrum are all joined in one rigid body.

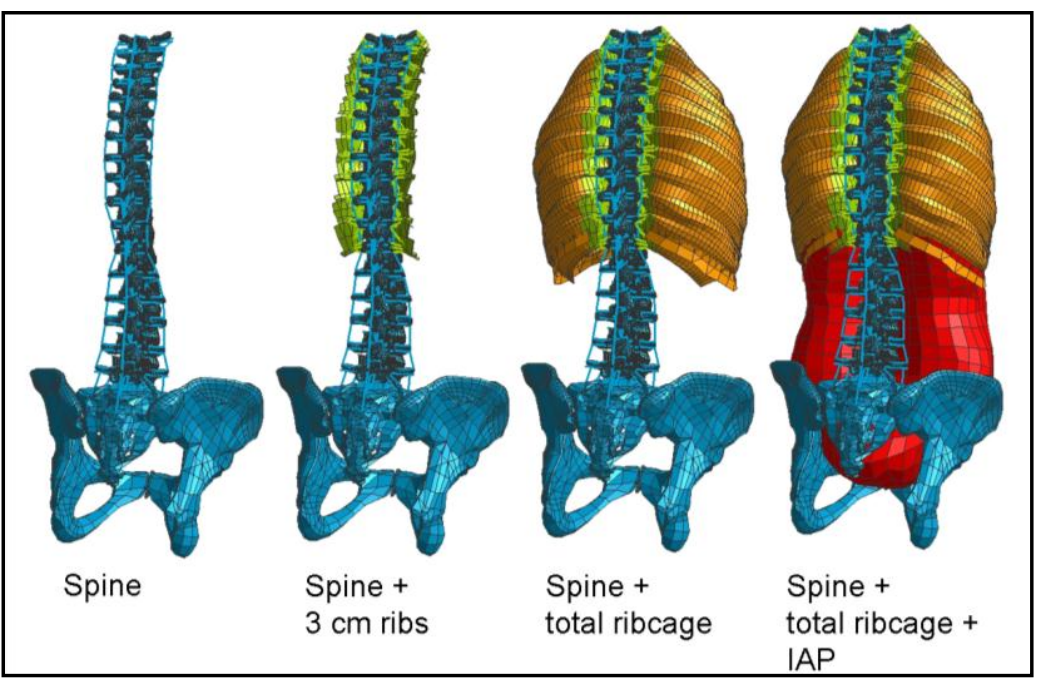

Figure 1: Finite element models: thoracolumbar spine (blue), including $3 \mathrm{~cm}$ ribs (green), including total ribcage (orange), and including intra-abdominal pressure (red). 
For the model parameters, related to the spine and the posterior part of the ribcage, we refer to our previous studies ${ }^{8-10}$. Anteriorly, the ribs and the sternum are connected by a set of cartilage elements. The geometry of the ribcage and abdominal cavity is based on the 5 th percentile female human model ${ }^{11}$.

The IAP is modelled as an incompressible volume in the shape of the intraabdominal cavity (figure 2 ) with an overpressure of $1 \mathrm{kPa}^{12-14}$.

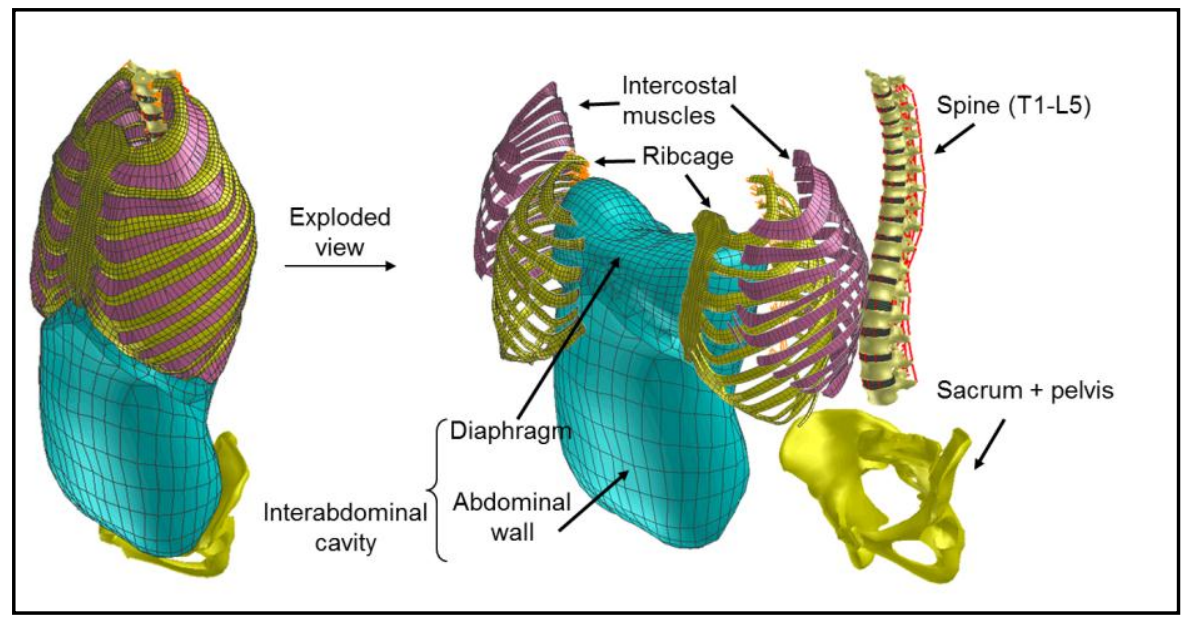

Figure 2: Anterior-lateral view of the trunk model (left). Exploded view (right): thoracolumbar spine, the ribcage, intercostal muscles, intra-abdominal cavity, sacrum and pelvis.

The upper part of the abdominal cavity is defined by the diaphragm. The lower part is the abdominal wall, which is defined as the outer skin layer of the abdomen and the pelvic outline, both with an offset of $10 \mathrm{~mm}$ (estimated thickness of the skin including the subcutaneous tissues) ${ }^{15,16}$. On the posterior side it is curved along the anterior side of the spine. The membrane layer surrounding the intra-abdominal cavity interacts with both the spine (connected to vertebrae T10 to L5 and sacrum) and the ribcage (contact between diaphragm and ribs). The orientation of the most important abdominal muscles (internal oblique and transversus abdominis ${ }^{17,18}$ ) are represented by a fibre reinforced material, with fibres oriented at respectively 80 degrees and 10 degrees with the horizontal. In this way, the direct effect of the IAP on the vertebrae is modelled, as well as the upward force of the diaphragm on the ribcage, the downward force on the pelvic floor and the (passive) stiffness of the abdominal muscles.

An overview of the used material parameters is presented in table 1. For all simulations PAM-CRASH software was used (ESI-group, France). 


\begin{tabular}{|c|c|c|}
\hline Structure & Material properties & Ref. \\
\hline Vertebrae & Rigid bodies & - \\
\hline Facet surfaces & $\begin{array}{l}\text { Stiffness increases with } \\
\text { compression depth }\end{array}$ & 8,9 \\
\hline Nucleus & $\mathrm{E}=1 \mathrm{MPa}, \nu=0.495$ & 19 \\
\hline Annulus matrix & $\mathrm{E}=2 \mathrm{MPa}, v=0.45$ & 20 \\
\hline Annulus fibres & $\mathrm{E}=450 \mathrm{MPa}$ & 21 \\
\hline Spinal ligaments & Three linear regions & 8 \\
\hline Costovertebral \& costotransverse ligaments & Seven linear regions & 10 \\
\hline Ribs & $\mathrm{E}=10.7 \mathrm{GPa}, v=0.3$ & 22 \\
\hline Cartilage between ribs and sternum & $\mathrm{E}=25 \mathrm{MPa}, v=0.45$ & 23 \\
\hline Intercostal muscles matrix & $\mathrm{E}=5 \mathrm{MPa}, v=0.4$ & - \\
\hline Intercostal muscles fibres & $\mathrm{E}=25 \mathrm{MPa}, \mathrm{v}=0.3$ & - \\
\hline Diaphragm membrane & $\mathrm{E}=35 \mathrm{MPa}, \mathrm{v}=0.3$ & - \\
\hline Abdominal membrane & $\mathrm{E}=5 \mathrm{MPa}, \mathrm{v}=0.45$ & - \\
\hline Abdominal membrane fibres & $\mathrm{E}=15 \mathrm{MPa}, v=0.3$ & - \\
\hline Costovertebral \& costotransverse contacts & Non-linear penalty contact & - \\
\hline
\end{tabular}

Table 1: Overview material properties

\subsubsection{Comparison of the finite element models with experimental data}

Although a true validation of adolescent models is not possible due to lack of adolescent experimental data, a comparison with experimental data of adult thoracic spines with $3 \mathrm{~cm}$ ribs, and of thoracic spines with the total ribcage ${ }^{7}$ can be made. The experimental adult data is then scaled by a factor 1.4 to account for the reported increased flexibility of the spine for adolescents ${ }^{8,24,25}$. Like in the experiments, the T12 was fully fixed and T1 was loaded with pure moments in flexion (1 Nm), extension $(1 \mathrm{Nm})$, lateral bending $(1 \mathrm{Nm})$ and axial rotation $(2.5 \mathrm{Nm})$.

\subsubsection{Influence of spine, ribcage and IAP on the stiffness of the trunk}

The stiffness in flexion, extension, lateral bending and axial rotation was compared for three models (thoracolumbar spine, spine including total ribcage, spine including ribcage and IAP). The L5 was fully fixed and T1 was loaded with pure moments in flexion, extension, lateral bending and axial rotation (all $2 \mathrm{Nm}$ ).

\subsection{Results}

\subsubsection{Comparison of the finite element models with experimental data}

In figure 3 the simulated results are presented together with the experimental data. The simulated ranges of motion lie within the range of the experimental data ${ }^{7}$, for both the spine including $3 \mathrm{~cm}$ ribs and the spine including the total ribcage. The differences between the movement directions are similar in the simulations and experimental data. 


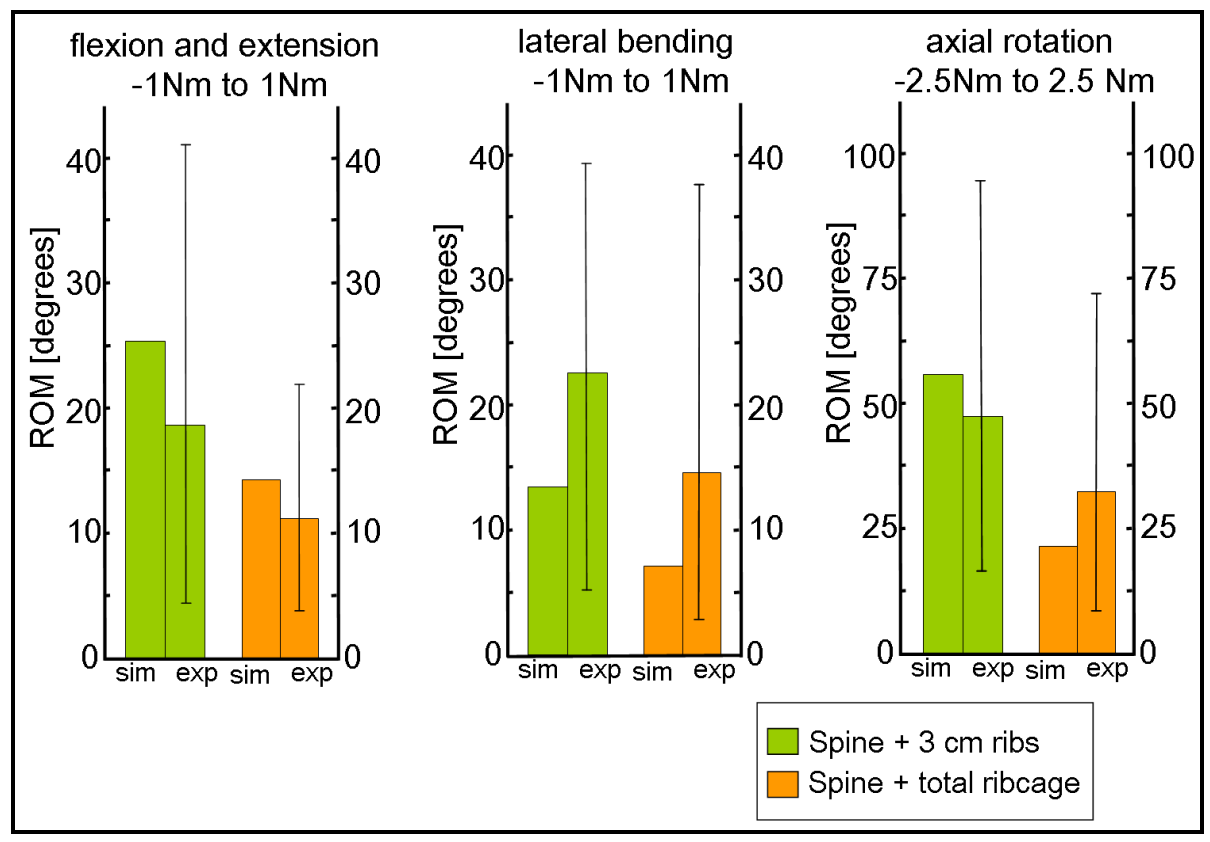

Figure 3: Simulated data (sim) are compared with experimental data (exp) for the mechanical behaviour of the thoracic spine including $3 \mathrm{~cm}$ ribs and total ribcage. For the experimental data the mean (bar) and range (error bar) are presented.

\subsubsection{Influence of spine, ribcage and IAP on the stiffness of the trunk}

In table 2 and figure 4 the results of the different simulated configurations are presented. The total ribcage mainly increases the stiffness of the thoracic spine and the IAP mainly increases the stiffness of the lower thoracic and lumbar spine (figure 4). These effects are seen for all movement directions.

\begin{tabular}{|rccc|} 
Movement direction & IAP & Ribcage & Spine \\
\hline Flexion & $50 \%$ & $25 \%$ & $25 \%$ \\
Extension & $55 \%$ & $22 \%$ & $23 \%$ \\
Lateral bending & $69 \%$ & $21 \%$ & $10 \%$ \\
Axial rotation & $65 \%$ & $19 \%$ & $16 \%$ \\
\hline
\end{tabular}

Table 2: The relative contribution of the IAP, ribcage and the spine to the total stiffness of the adolescent trunk. 

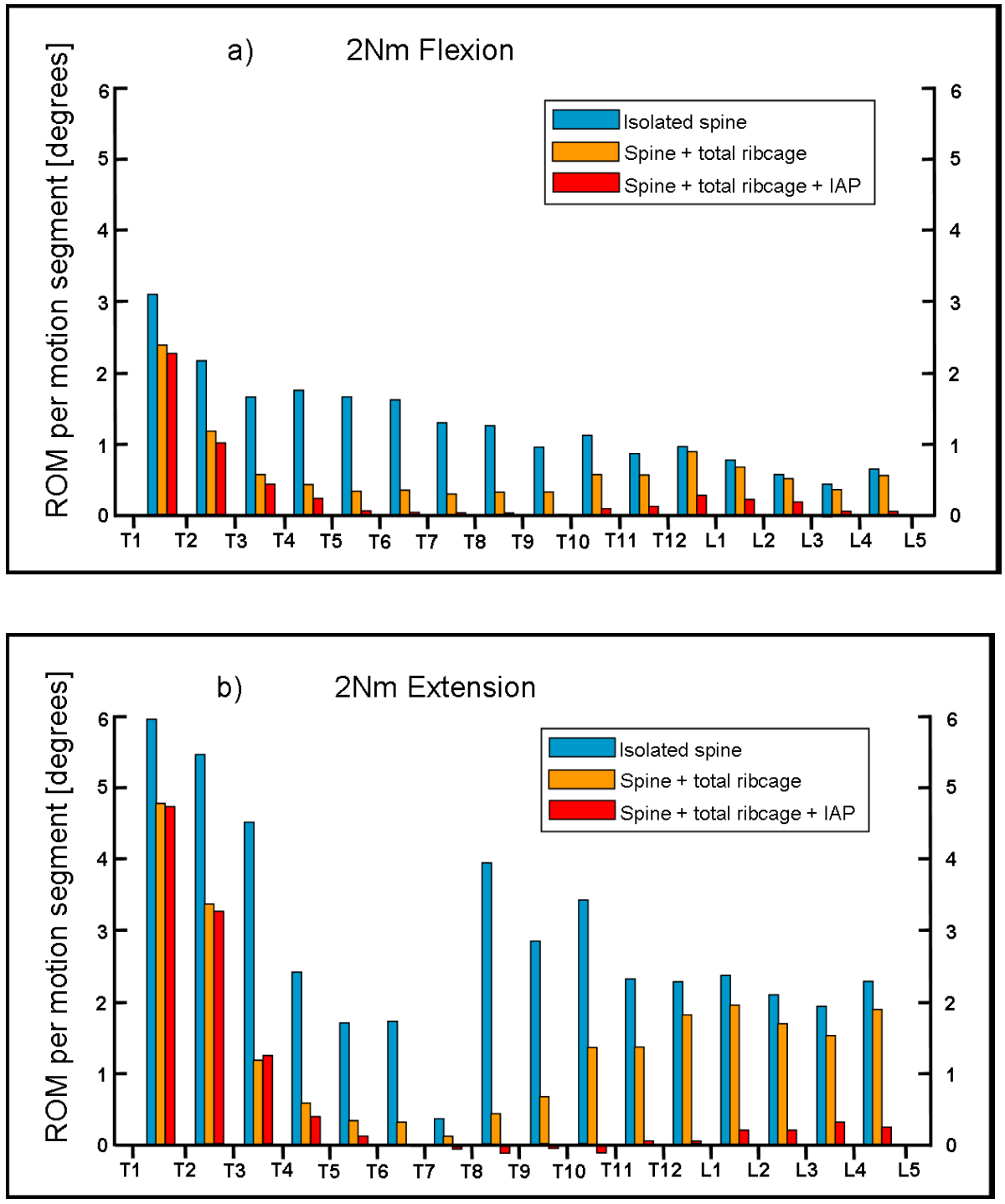

Figure 4: The motion of the isolated spine, spine and the total ribcage, spine and total ribcage and IAP, is compared when loaded with $2 \mathrm{Nm}$ flexion (a), $2 \mathrm{Nm}$ extension (b), $2 \mathrm{Nm}$ lateral bending(c) and $2 \mathrm{Nm}$ axial rotation (d). The range of motion (ROM) is given for each motion segment. 

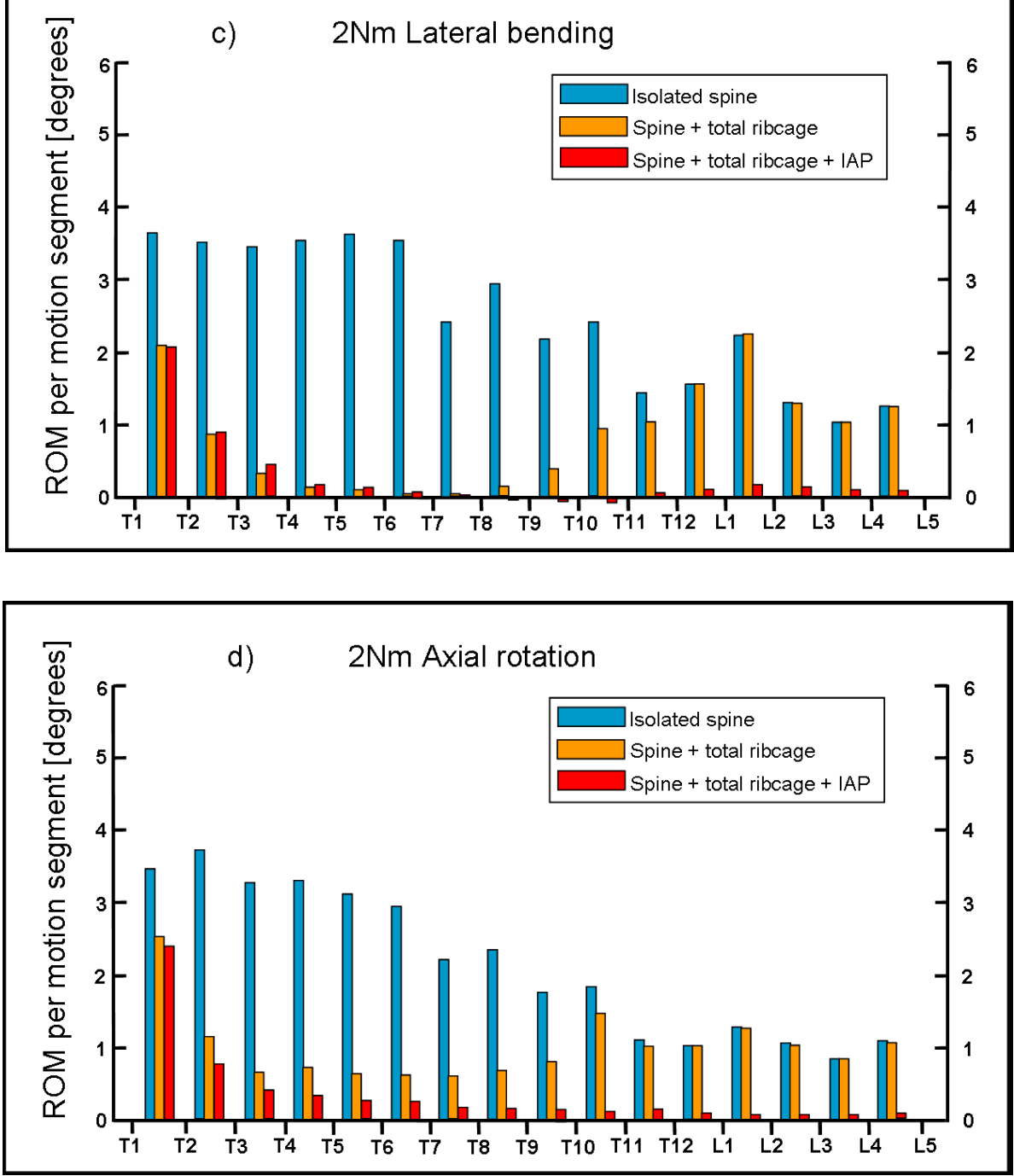

Figure 4: The motion of the isolated spine, spine and the total ribcage, spine and total ribcage and IAP, is compared when loaded with $2 \mathrm{Nm}$ flexion (a), $2 \mathrm{Nm}$ extension (b), $2 \mathrm{Nm}$ lateral bending(c) and $2 \mathrm{Nm}$ axial rotation (d). The range of motion (ROM) is given for each motion segment. 


\subsection{Discussion and conclusion}

The natural anatomical variation between subjects results in a large range in the experimental data that is used for validation ${ }^{7}$. Our models, representing only one of these anatomical variations, show results that lie within this range for both the spine and the spine including the ribcage and is therefore satisfactory. Nonetheless, the relative contribution of the ribcage does differ between our adolescent model and the experimental study in adults ${ }^{7}$. The experimental study found low effects of the ribcage in lateral bending (35\%) and axial rotation (31\%), while we found $91 \%$ and $159 \%$ respectively. In the experimental study the largest influence (40\%) for the ribcage was found for the combined flexion and extension movement, while we found $78 \%$. It is unclear whether differences between our simulation and this study can be related to differences between adults and adolescents (other than the used overall scaling), the age of the tested specimen (average 72 years), differences in loading conditions (it is unclear whether the applied loading range in the experiments was for unidirectional or bidirectional testing) or are due to modelling assumptions.

When comparing the results of our total trunk model to in vivo experiments in adolescents ${ }^{26}$, comparable results for the average flexion bending stiffness (EI) of the adolescent spine are found: $8 \mathrm{Nm}^{2}$ and $8.8 \mathrm{Nm}^{2}$ respectively. Unfortunately, no experimental results for other movement directions are available.

For the current study we opted for an explicit solver. This choice was based on the availability of a good starting model and the large number of elements in the final model. An unfavourable side-effect of explicit solvers lies in the errors for the static equilibrium condition that can develop. Therefore the support moment and forces were verified. For the isolated spine models errors in the support moment were $0 \%$, for the models including the total ribcage the errors were up to $25 \%$, for the model including the IAP the errors were less than 5\%. Although various methods were used to reduce these errors (timing of the damping, time step, hour glass reduction, et cetera), a further reduction of these errors could not be achieved. It also remained unclear why the errors are largest in the model including the total ribcage. The models, in which the errors could successfully be reduced, showed that the errors only influenced the movement of the lumbar and low-thoracic vertebrae (T8-L5). Based on the same analyses we estimate that the maximum error of $25 \%$ in the local moments results in a $20 \%$ error of the movement of T8-L5. Although we do not expect the qualitative conclusions of this study to change due to the errors, the quantitative effects need further study. For this reason, we consider our results preliminary.

In the current study an adolescent model was used. Although the size of the ribcage is still changing during adolescent growth, a radiographic study ${ }^{27}$ showed that the cross-sectional shape of the ribcage and abdomen does not change during adolescence, nor does the orientation of the ribs. Therefore the qualitative results should be similar for adults and adolescents.

Our results show that both the ribcage and intra-abdominal pressure have a large effect on the stiffness of the trunk (19-25\% and $50-69 \%$ respectively). We also found that the ribcage mainly influences the mid-thoracic region (T4-T10), while the IAP mainly influences the low-thoracic and lumbar regions (T6-L5). This seems anatomically logical, since the transverse cross-sectional area of the ribcage is 
largest for rib 4 through 9 and the diaphragm (the upper border of the abdominal cavity) attaches to the 6th rib.

As stated in the introduction, the exact working mechanism of the IAP is unclear. Various theories have been proposed: ranging from generation of an extensor moment due to a downward force on the pelvic floor and an upward force on the diaphragm ${ }^{28}$; to limitation of the intervertebral rotation and translation due to the pressure on the spine ${ }^{29}$; to maintaining the hoop-like geometry of the muscles, necessary for providing tension ${ }^{30}$. Our current results suggest a role of both the lumbar vertebrae and the diaphragm, but further studies are needed to test these various theories.

\section{Acknowledgement}

This study was financially supported by the Dutch Technology Foundation, STW, applied science division of NWO and the Technology Program of the Ministry of Economic Affairs.

We thank Christine Obbink-Huizer for her help with the development of the ribcage model and Muriel Beaugonin for her help with the development of the model of the intra-abdominal cavity. We thank Nico Verdonschot and Timo Meinders for the useful discussions.

We thank ESI Group for the availability of the $5^{\text {th }}$ percentile female model, for the use of their software (PAM-CRASH) and support. 


\section{References}

[1] M. Essendrop, T.B. Andersen, B. Schibye, 'Increase in spinal stability obtained at levels of intra-abdominal pressure and back muscle activity realistic to work situations'. Applied Ergonomics Vol. 33, pp. 471-476, 2002.

[2] J. Cholewicki, K. Juluru, A. Radebold, M.M. Panjabi, S.M. McGill, 'Lumbar spine stability can be augmented with an abdominal belt and/or increased intra-abdominal pressure'. European Spine Journal Vol. 8, pp. 388-395, 1999.

[3] N. Arjmand, A. Shirazi-Adl, 'Role of intra-abdominal pressure in the unloading and stabilization of the human spine during static lifting tasks'. European Spine Journal Vol. 15, pp. 1265-1275, 2006.

[4] K. Daggfeldt, A. Thorstensson, 'The mechanics of back-extensor torque production about the lumbar spine'. J Biomech Vol. 36, pp. 815-825, 2003.

[5] I.A.F. Stokes, M.G. Gardner-Morse, S.M. Henry, 'Intra-abdominal pressure and abdominal wall muscular function: Spinal unloading mechanism'. Clin Biomech Vol. 25, pp. 859-866, 2010.

[6] T. Andriacchi, A. Schultz, T. Belytschko, J. Galante, 'A model for studies of mechanical interactions between the human spine and rib cage'. J Biomech Vol. 7, pp. 497-507, 1974.

[7] R. Watkins, R. Watkins, L. Williams, S. Ahlbrand, R. Garcia, A. Karamanian, L. Sharp, C. Vo, T.P. Hedman, 'Stability provided by sternum and rib cage in the thoracic spine.'. Spine Vol. 30, pp. 1283-1286, 2005.

[8] G.J.M. Meijer, J. Homminga, E.E.G. Hekman, A.G. Veldhuizen, G.J. Verkerke, 'The effect of three-dimensional geometrical changes during adolescent growth on the biomechanics of a spinal motion segment'. J Biomech Vol. 43, pp. 1590-1597, 2010.

[9] G.J.M. Meijer, J. Homminga, A.G. Veldhuizen, G.J. Verkerke, 'Influence of interpersonal geometrical variation on spinal motion segment stiffness: Implications for patient-specific modeling'. Spine Vol. 36, pp. E929-E935 2011.

[10] G.J.M. Meijer, J. Homminga, G.J. Verkerke, 'Influence of costovertebral joints on the stiffness of the spine'. submitted.

[11] S. Na, S. Sah, D. Hwang, H.Y. Choi, I. Lee, J. Lee, 'Numerical simulation of out-of-position driver side airbag deployment with small female model'. JSAE Annual Congress Vol. 8, pp. 5-8, 2003.

[12] A. Schultz, G. Andersson, R. Ortengren, K. Haderspeck, A. Nachemson, 'Loads on the lumbar spine. Validation of a biomechanical analysis by measurements of intradiscal pressures and myoelectric signals'. J Bone Joint Surg Vol. 64-A, pp. 713-720, 1982.

[13] M. Essendorp Significance of intra-abdominal pressure in work related trunk-loading. PhD thesis, National Institute of Occupational Health/ Copenhagen, Denmark, 2003.

[14] A.L. Nachemson, G.B.J. Andersson, A.B. Schultz, 'Valsalva maneuver biomechanics: effects on lumbar trunk loads of elevated intraabdominal pressures'. Spine Vol. 11, pp. 476479., 1986.

[15] H. Shin, M.J. Kim, 'Subcutaneous tissue thickness in children with type 1 diabetes'. Journal of Advanced Nursing Vol. 54, pp. 29-34, 2006.

[16] M.A. Gibney, C.H. Arce, K.J. Byron, L.J. Hirsch, 'Skin and subcutaneous adipose layer thickness in adults with diabetes at sites used for insulin injections: implications for needle length recommendations'. Current Medical Research and Opinion Vol. 26, pp. 1519-1530, 2010. [17] A. Cresswell, A. Thorstensson, 'Changes in intra-abdominal pressure, trunk muscle activation and force during isokinetic lifting and lowering'. European Journal of Applied Physiology and Occupational Physiology Vol. 68, pp. 315-321, 1994.

[18] A.G. Cresswell, H. Grundström, A. Thorstensson, 'Observations on intra-abdominal pressure and patterns of abdominal intra-muscular activity in man'. Acta Physiologica Scandinavia Vol. 144, pp. 409-418., 1992.

[19] A.P. Dooris, V.K. Goel, N.M. Grosland, L.G. Gilbertson, D.G. Wilder, 'Load-sharing between anterior and posterior elements in a lumbar motion segment implanted with an artificial disc'. Spine Vol. 26, pp. E122-E129, 2001.

[20] F. Lavaste, W. Skalli, S. Robin, R. Roy-Camille, C. Mazel, 'Three-dimensional geometrical and mechanical modelling of the lumbar spine'. J Biomech Vol. 25, pp. 1153-1164., 1992. 
[21] V.K. Goel, B.T. Monroe, L.G. Gilbertson, P. Brinckman, 'Interlaminar shear stresses and laminae separation in a disc. Finite element analysis of the L3-L4 motion segment subjected to axial compressive loads'. Spine Vol. 20, pp. 689-698., 1995.

[22] J.D. Stitzel The role of local material properties in modeling fracture tolerance of the human thorax. PhD thesis, Viriginia polytechnic institute and state university/ Blacksburg, Virginia, 2003.

[23] H. Yamada (1970) Strength of biological materials. 1st edn. Williams and Wilkins, Baltimore (MD, USA)

[24] S. Kumaresan, N. Yoganandan, F.A. Pintar, D.J. Maiman, S. Kuppa, 'Biomechanical study of pediatric human cervical spine: a finite element approach.'. J Biomech Eng Vol. 122, pp. 60$71,2000$.

[25] J. Ouyang, Q. Zhu, W. Zhao, Y. Xu, W.J. Chen, S. Zhong, 'Biomechanical assessment of the pediatric cervical spine under bending and tensile loading'. Spine Vol. 30, pp. E716-E723, 2005.

[26] P.J.M. Scholten, A.G. Veldhuizen, 'The bending stiffness of the trunk'. Spine Vol. 11, pp. 463-467., 1986.

[27] P. Openshaw, S. Edwards, P. Helms, 'Changes in rib cage geometry during childhood'. Thorax Vol. 39, pp. 624-627, 1984.

[28] J. Cholewicki, K. Juluru, S.M. McGill, 'Intra-abdominal pressure mechanism for stabilizing the lumbar spine'. J Biomech Vol. 32, pp. 13-17, 1999.

[29] S.M. McGill, R.W. Norman, 'Reassessment of the role of intra-abdominal pressure in spinal compression '. Ergonomics Vol. 30, pp. 1565-1588., 1987.

[30] S.M. McGill, R.W. Norman (1993) Low back biomechanics in industry: the prevention of injury through safe lifting. In: Current issues in biomechanics. Human Kinetics Publishers, Champaign, IL., pp 69-120 


\section{Chapter 7}

Is scoliosis induction a good model for scoliosis correction?

G.J.M. Meijer, M. Wessels, E.E.G. Hekman, J. Homminga, G.J. Verkerke

To be submitted for publication 


\begin{abstract}
Scoliosis is a deformity of the spine and trunk, mainly characterized by a lateral deviation of the spinal column in combination with axial rotation of the vertebrae. In the current project, we developed a new scoliosis correction implant, which will apply small correction forces over a longer period. Due to the visco-elastic properties and adaptation of the soft tissues of the spine, a complete correction can be obtained over time. By applying both bending forces and torsion, a correction of the lateral deviation and axial rotation is achieved. The focus of this study is on the effects of the torsional correction implant.

As part of the development of new implants, the effects of the implants are tested in animal experiments. However, as scoliosis does not occur in animals, these tests study scoliosis induction rather than scoliosis correction. Methods for scoliosis correction in humans are thus tested by inducing scoliosis in animals.
\end{abstract}

In this chapter, we compared the effects of pure torsion moments on a healthy spine in which scoliosis is induced to pure torsion moments on a scoliotic spine in which scoliosis is corrected. The short term and long term -including visco-elasticity and adaptation of soft tissues- effects on lateral and axial deformity are analysed. Comparison of scoliosis correction and induction in our models showed that the mechanical effects of a torsion loading on a healthy spine differ from those on a scoliotic spine. Although both showed effects in the axial rotation and lateral deformity, and no effects on the sagittal shape, the effects in the sagittal plane were much higher in the scoliosis correction simulations than in the scoliosis induction simulations. The use of scoliosis induction in animals to predict the outcome of scoliosis correction in humans might therefore not be a valid method, although alternatives are hard to find.

The simulations also show that the long-term correction of the new implant will be considerable. Therefore, a long-term correction of a mild scoliosis with relative low moments (1.5 Nm torsion) seems feasible. Our result also showed that torsion moments can be used to correct the lateral deformity in scoliosis. The amount of correction that is achieved of course depends on the patient-specific situation; both the patient-specific stiffness of the trunk and the scoliotic shape will influence the correction outcome. 


\subsection{Introduction}

Scoliosis is a deformity of the spine and trunk, mainly characterized by a lateral deviation of the spinal column in combination with axial rotation of the vertebrae. The most common form of scoliosis is Adolescent Idiopathic Scoliosis (AIS); mild forms of AIS can be found in 2 to 3 per cent of the children between 10 and 16 year ${ }^{1-4}$.

The current treatment of scoliosis depends on the severity of the curve (Cobb angle), the remaining growth (age) and the progression of the curve (increase of the Cobb angle) ${ }^{5-7}$.

In mild cases with little curve-progression, the patient is simply monitored, and often is given physiotherapy and exercises. In mild and moderate scoliosis with progression of the curve, bracing is used to limit progression of the scoliosis. Major disadvantage of this treatment is that curve progression will reoccur when the brace is no longer used and full correction is not achieved ${ }^{8,9}$.

When Cobb-angles exceed 40 degrees and the curve is progressive, metal implant systems are used to correct the deformity and fuse the spine in its corrected state. Major disadvantages of this form of treatment are that it cannot start until growth is (almost) complete and that the mobility of the spine is greatly reduced.

A new correction implant, in which the vertebrae will not fuse, is developed for mild but progressive scoliotic curves. Such an implant will have multiple advantages. First, the implant can be used to correct scoliosis while the growth is still ongoing. Second, due to the earlier application of the correction, the deformity will be smaller, thus requiring smaller forces than in current surgical treatments. Third, the preservation of the mobility of the spine is an improvement of the quality of life for the patients. Fourth, after full correction is achieved, the implant can be removed and a normal, fully functional spine is the result.

As part of the development of new implants, the effects of scoliosis correction implants are tested in animal experiments ${ }^{10-12}$. However, as scoliosis does not occur in animals, these tests study scoliosis induction rather than scoliosis correction. Methods for scoliosis correction in humans are thus tested by inducing scoliosis in animals. While the differences in spinal biomechanics of humans and animals have been studied extensively ${ }^{13-16}$, the differences between scoliosis induction and correction have not.

From a mechanical point of view, the relation between scoliosis correction and induction is not straight-forward. Therefore, we studied the mechanical behaviour of a healthy spine in which scoliosis is induced and of a scoliotic spine in which scoliosis is corrected, to analyse whether these in vivo scoliosis induction experiments are a good representation for scoliosis correction. For this we used numerical models of the adolescent trunk. 


\subsection{Methods}

\subsubsection{Finite element models}

To evaluate the effect of various treatment methods and implants, numerical models are often used ${ }^{17-20}$. Since scoliosis affects large parts of the thoracolumbar spine ${ }^{2}$ $4,21,22$ and the ribcage ${ }^{23}$, these models should represent this total thoracolumbar spine and ribcage. In current complete spine models, however, the motion segments are oversimplified. Often a single stiffness matrix represents the total intervertebral disc, spinal ligaments and sometimes even parts of the ribcage. Especially for scoliosis research, this representation is not sufficient, since changes of this stiffness matrix for the deformed geometry in scoliosis are not straight-forward and the deformations (in both scoliosis progression and correction) are large. Therefore we developed a new finite element model that represents the essential biomechanics of the adolescent spine, ribcage and trunk on a macroscopic level including nonlinearity in both geometry and material properties.

This numerical model of the trunk is based on our previously developed models of a lumbar motion segment 24,25 and of thoracic multi-segments ${ }^{26}$. We developed 2 models of an adolescent trunk; one "healthy" and one scoliotic (figure 1). Both models contain the thoracolumbar spine, ribcage, passive behaviour of intercostal, abdominal and dorsal muscles, as well as intra-abdominal pressure.

A detailed description of the healthy model and its validation has been given in chapter 6 .

The scoliotic model is not patient-specific, but represents an average adolescent patient for whom the new scoliosis correction implant is suitable. This patient would currently have been treated with a brace: a moderate $\left(25^{\circ}-45^{\circ} \mathrm{Cobb}\right)$ but progressive scoliosis.

The most common types of scoliosis are single thoracic and double thoracolumbar curves $^{2-4,21,22}$. Since the correction of a double curve would in essence be the correction of two single curves, we focussed on the single thoracic curve.

The most frequent location of the apex in a single thoracic curve is T8 ${ }^{27}$. The precise relation between the deformity in the frontal plane and the axial rotation is not clear, and is likely patient-specific, but it has been suggested that progressive curves have more axial rotation ${ }^{28}$. Based on this, a scoliotic model was created with a single thoracic curve (apex at T8), with a Cobb angle of $32^{\circ}$ and an axial rotation of the apex of $24^{\circ}$. The scoliosis is created by prescribing a lateral translation and axial rotation at the vertebrae in the scoliotic curve (T4-T11), while the lowermost vertebra (L5) was fully fixed and the uppermost vertebra (T1) was fixed for translations in the transverse plane.

As we modelled the complete trunk, these displacements of the vertebrae resulted in a natural deformation of the entire trunk. 


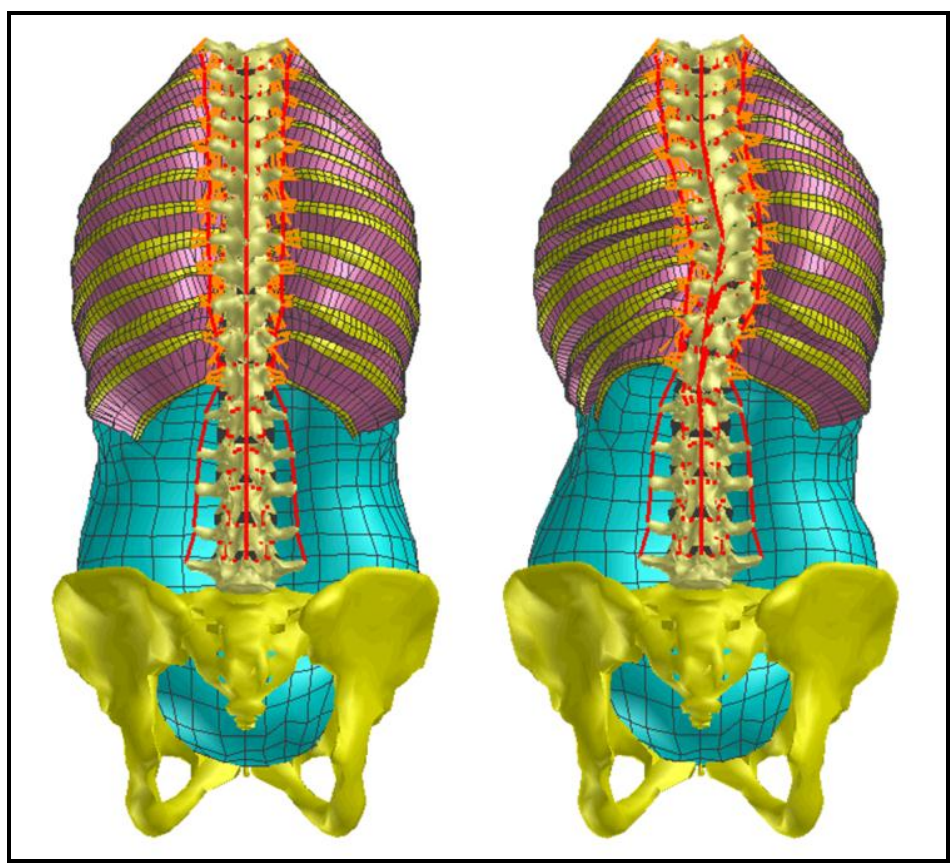

Figure 1 Healthy (left) and scoliotic (right) model.

A full adaptation of the soft tissues to the scoliotic position is assumed. A previous study described the mechanobiological process of adaptation of ligaments and tendons during growth, whereby the ligaments continuously adapt to maintain a certain physiological strain-level ${ }^{29}$. In this study, this was achieved by first resetting all stresses and strains in the deformed position to zero, after which the same prestrain as in the healthy situation ${ }^{24}$ are applied to the ligaments.

Since bone deformation occurs in later, and more severe, stages of scoliosis ${ }^{23,30}$, the vertebrae and ribs are assumed undistorted and therefore all wedging and axial rotation is located in the intervertebral discs and altered connection between the ribs and vertebrae. Also, the contact definition of the facet joints is not altered and initial penetrations are not removed. The assumption of only modelling soft tissue adaptation and no bone remodelling is thus consistent with the targeted patient group of mild cases of scoliosis.

\subsubsection{New implant}

The newly developed implant for scoliosis corrections can correct mild, but progressive forms of scoliosis, by applying small correction forces over a longer period of time. To correct the three-dimensional behaviour of the deformity, the lateral and axial rotation can be corrected independently by different implants. In the current study, the focus is on the implant that aims to correct the axial deformity of the scoliotic spine and trunk. 
Due to the visco-elastic behaviour and adaptation of the soft tissues of the human spine and trunk, large amounts of additional correction are possible after the operation. This effect was previously described ${ }^{31}$. However, unlike our newly developed implant, that implant resulted in fusion of the spine.

Pilot measurements with a prototype of the new implant showed that the implant will deliver $1.5 \mathrm{Nm}$ to the human adolescent spine. Due to the visco-elastic behaviour of the spine and trunk this $1.5 \mathrm{Nm}$ will reduce over time ${ }^{31-33}$, although the exact reduction for the new system is yet unknown.

\subsubsection{Simulations}

Scoliosis induction was simulated in the healthy model. A torsional moment of -1.5 $\mathrm{Nm}$ was applied at the T8 and $+0.75 \mathrm{Nm}$ was applied at both T4 and T11. In the simulations the L5 was fully fixed, and translations of T1 in the transversal plane were restricted (rotations and axial translation were free).

Scoliosis correction in the scoliotic model was modelled in the same manner. A torsional moment of $+1.5 \mathrm{Nm}$ was applied at the T8, and $-0.75 \mathrm{Nm}$ was applied at the T4 and T11. L5 was fully fixed, and the translations of T1 in the transversal plane were restricted (rotations and axial translations were free).

The effects of the applied moment on the axial rotation, the Cobb angle, and kyphosis of the spine were analysed. To analyse the long term effects of both the scoliosis correction and induction, due to adaptation of the soft tissues and viscoelastic behaviour, an extra iteration step was performed. In these sequential steps, again an adaptation of the soft tissues is assumed, whereby the ligaments will adjust until a certain physiological strain-level is achieved ${ }^{29}$. This was modelled by resetting all strains and stresses in all tissues to zero, and reapplying the initial prestrain in the spinal ligaments ${ }^{24}$.

The deformity, achieved in the first iterative step, will result in a reduced loading by the implant. The moment on the apex in the second iteration step was therefore set to $1.4 \mathrm{Nm}$ (and $-0.7 \mathrm{Nm}$ on the T4 and T11). In reality, this loop of applying a correction moment, changing geometry, strain relaxation, and decrease of the applied moment, would continue until the spine no further deforms when the load is adapted. Since the actual qualitative aspects of the process (amount of stress relaxation, time scale, and influence on applied loading) are all unknown, only two iteration steps were analysed.

\subsection{Results}

\subsubsection{Scoliosis induction}

A comparison of the long term and short term scoliosis induction simulations showed that the relaxation of the soft tissues results in an increased scoliosis over time (table 1).

The results show that the axial rotation between the upper fixation and the apex was about two times as large as the rotation between the apex and the lower fixation. The pure torsion moment also induced a small Cobb angle, but did not alter the kyphosis. The second simulation step increased the axial rotations, but not the Cobb angle and the kyphosis angle. 


\begin{tabular}{|rcc|} 
& $\begin{array}{c}\text { short term } \\
\text { (step 1) }\end{array}$ & $\begin{array}{c}\text { Long term } \\
\text { (step 1+2) }\end{array}$ \\
\hline axial rotation between T4 and T8 & $-2.9^{\circ}$ & $-5.5^{\circ}$ \\
axial rotation between T8 and T11 & $1.6^{\circ}$ & $3.1^{\circ}$ \\
change in Cobb angle & $0.4^{\circ}$ & $0.4^{\circ}$ \\
change in kyphosis between T4 and T8 & $0^{\circ}$ & $0^{\circ}$ \\
\hline
\end{tabular}

Table1: Scoliosis induction results for short and long term simulations. T4=upper fixation of implant, T8=apex and middle fixation of implant, T11=lower fixation of implant.

\subsubsection{Scoliosis correction}

A comparison of the long term and short term scoliosis correction simulations showed that the relaxation of the soft tissues results in an increased correction over time (table 2).

The scoliosis correction simulations did not only show a correction of the axial rotation by the applied torsion moment, but also a correction of the Cobb angle. Both the correction of the axial rotation and Cobb angle increased with the second simulation step, although the amount of correction due to this second step was less than in the first step. Again, the torsion moment had no effect on the kyphosis.

\begin{tabular}{|rcc|}
\hline & $\begin{array}{c}\text { short term } \\
\text { (step 1) }\end{array}$ & $\begin{array}{c}\text { Long term } \\
\text { (step 1+2) }\end{array}$ \\
\hline axial rotation between T4 and T8 & $2.6^{\circ}$ & $3.8^{\circ}$ \\
axial rotation between T8 and T11 & $-1.9^{\circ}$ & $-2.9^{\circ}$ \\
change in Cobb angle & $-1.6^{\circ}$ & $-2 .^{\circ}$ \\
change in kyphosis between T4 and T8 & $0^{\circ}$ & $0^{\circ}$ \\
\hline
\end{tabular}

Table 2 Scoliosis correction results for long term and short term simulations. T4=upper fixation of implant, T8=apex and middle fixation of implant, $T 11=$ lower fixation of implant.

\subsubsection{Comparison of scoliosis correction and induction}

A comparison between the scoliosis correction and induction is made for both the short term (table 3) and long term (table 4) effects.

The short term effects on the axial rotation are very similar for the correction and induction simulations. There is also no effect on the kyphosis for either case. However, the effect on the Cobb angle is different. In the scoliosis correction simulation, the effect on the Cobb angle is considerable larger than that in the scoliosis induction simulation ( $1.6^{\circ}$ correction versus $0.4^{\circ}$ induction).

For the long term effects, again the axial rotations in the induction and correction simulations are comparable, although the effects in induction are larger. In the second iterative step the change in Cobb angle was remarkably less for the induction simulation compared to the correction simulation $\left(0.4^{\circ}\right.$ induction versus $2.3^{\circ}$ correction). 


\begin{tabular}{|rcc|} 
& $\begin{array}{c}\text { induction } \\
\text { (step 1) }\end{array}$ & $\begin{array}{c}\text { correction } \\
\text { (step 1) }\end{array}$ \\
\hline axial rotation between T4 and T8 & $-2.9^{\circ}$ & $2.6^{\circ}$ \\
axial rotation between T8 and T11 & $1.6^{\circ}$ & $-1.9^{\circ}$ \\
change in Cobb angle & $0.4^{\circ}$ & $-1.6^{\circ}$ \\
change in kyphosis between T4 and T8 & $0^{\circ}$ & $0^{\circ}$ \\
\hline
\end{tabular}

Table 3 Comparison between short term scoliosis induction and correction. T4=upper fixation of implant, T8=apex and middle fixation of implant, T11=lower fixation of implant.

\begin{tabular}{|rcc|} 
& $\begin{array}{c}\text { induction } \\
(\text { step 1+2) }\end{array}$ & $\begin{array}{c}\text { correction } \\
(\text { step 1+2) }\end{array}$ \\
\hline axial rotation between T4 and T8 & $-5.5^{\circ}$ & $3.8^{\circ}$ \\
axial rotation between T8 and T11 & $3.1^{\circ}$ & $-2.9^{\circ}$ \\
change in Cobb angle & $0.4^{\circ}$ & $-2.3^{\circ}$ \\
change in kyphosis between T4 and T8 & $0^{\circ}$ & $0^{\circ}$ \\
\hline
\end{tabular}

Table 4 Comparison between scoliosis long term induction and correction. T4=upper fixation of implant, T8=apex and middle fixation of implant, T11=lower fixation of implant.

\subsection{Discussion and conclusion}

In the current study, the results for scoliosis induction simulations and scoliosis correction simulations are compared, to assess the validity of animal scoliosis induction experiments. Some limitations of the model and method need to be discussed.

First of all, material parameters and validation studies for the adolescent model are difficult to find. Although some in vivo studies in adolescents exist, these are hampered by the uncontrollability of the loading condition due to muscle activation. Although the mechanical behaviour of the total healthy model is validated with the available in vivo experiments, the actual material input data are based on studies in adults, and only the geometrical parameters are truly adolescent. This also applies to the scoliotic adolescent model: no studies into material parameters or mechanical experiments have been published.

Second, the current scoliotic model represents an average scoliosis and does not represent any specific patient. The chosen case represents the geometry of a patient that would be suitable for implantation with the new system, but since the true cause of idiopathic scoliosis is unknown, the current way of modelling scoliosis may not represent the true underlying effects of scoliosis.

Third, the assumption of the time scale of the stress release after the modelling of the scoliotic form and after each iteration step in scoliosis induction and correction. The previously described and validated mechanobiological process of adaptation of ligaments and tendons, aims to maintain a constant physiological strain-level ${ }^{29}$. This was modelled by first resetting all stresses and strains in the deformed position to zero, after which the same prestrain as in the healthy situation ${ }^{24}$ are applied to the ligaments. The limitation of this modelling lies in the unknown time scale of this adaptation. Furthermore it is unknown whether the adaptation in scoliotic patients is equal to the adaptation in healthy subjects. 
Fourth, in the model the facet joints are represented by parallel, planar surfaces, instead of a geometry that is curved in $3 \mathrm{D}$, as it is in reality. Our simulations into the effects of the facet geometry on the behaviour of an L3-L4 motion segment showed that representation by a 3D curved surface resulted in earlier contact between the facet surfaces. However, as the initial contact forces are very small and increase progressively, the effects on the load-displacement curve of the motion segment are small. Since the thoracic facet joints are in reality more planar than the lumbar ones, it is expected that these effects are even less for the thoracic region, and the current assumption therefore seems valid when analysing the kinematics of the thoracolumbar spine.

This study showed that the mechanical effects of a torsion loading on a healthy and scoliotic spine are not similar. Although both showed an effect in the rotation angle and sagittal plane, and no effects on the kyphosis, the effects in the sagittal plane were much higher in the scoliosis correction simulations than in the scoliosis induction simulations. This suggests a different relation between the applied loading and coupled movements for scoliotic and healthy spines. Although some studies into the coupled movements of the scoliotic relative to the normal spine exist ${ }^{34-36}$ all these studies analyse the opposite phenomena: what is the (coupled) axial rotation when a lateral bending moment is applied. Two studies ${ }^{34,35}$ found similar orientations of the coupling behaviour for the scoliotic and the healthy spine, but an increased amount of coupling was found in (mild) scoliotic spines. A third study ${ }^{36}$ found a decoupling between the axial rotation and lateral bending behaviour, but explicitly stated that this holds for severe scoliosis. Since our focus lies on mild scoliosis, an increased coupling between lateral bending and axial rotation is more likely. The explanation given by both studies are related to the facet joints: Veldhuizen and Scholten ${ }^{35}$ show a high relation between the altered orientation of the facet joints in the sagittal plane and the coupling behaviour, Farahpour ${ }^{34}$ explains that the coupling movement is necessary to facilitate the motion by unlocking the facet joints.

In the current study, the orientation of the facet joints in the (global) sagittal plane will be different in the two models, since an axial deformity occurred in the scoliotic model. A different coupling between the applied torsion moment and the resulting lateral bending in the healthy and scoliotic model is therefore likely and could very well explain the differences in the sagittal plane found between the correction and induction simulations that are found in this study.

Another difference between the scoliosis induction and correction simulations was found for the long term behaviour: the extra rotation due to the second iteration step is larger for scoliosis induction than for the scoliosis correction.

This could also be explained by differences in the working of the facet joints. Due to the limited rotation there is no contact between the facet surfaces in the scoliosis induction simulations; the facet joints generate no force in neither the first nor the second step of the induction. In scoliosis correction, the facet joints generate forces that help the correction. These forces are smaller in the second simulation (due to the smaller axial rotation and the non-linear contact definition), resulting in a smaller correction found for the second step.

The clinical implication of the differences between the scoliosis correction and induction simulations is that the currently used in vivo animal experiments in which scoliosis is induced to prove the principle of scoliosis correction has some limitations. 
Unfortunately we cannot offer a full solution. To improve the usability of the results of the animal experiments, it might be better to first induce scoliosis in the animals. Ethical committees currently consider two operations in the same animal unacceptable. But apart from the ethical issues, even assuming that a representative deformity can be modelled (as we also do in our model study), it is questionable if one could induce this deformity without unknown side-effects in an animal. Although some proposals for animal models of scoliosis are found in literature ${ }^{37-39}$ it is unclear if the true underlying effects of scoliosis are present.

Besides the comparison between the scoliosis correction and induction, this study also demonstrates the effectivity of the new scoliosis correction implant. Although the short term correction of the new scoliosis correction implant is small, the long-term correction is considerable, especially when we consider that only two iteration steps are simulated. A long term correction of a mild scoliosis with relative low moments (1.5 Nm torsion) thus seems realistic. The required time for such a correction cannot be determined from simulations and should be experimentally determined.

The results of the current study also showed that a torsion implant can also correct the Cobb angle in scoliotic patients. The ratio between these corrections of course depends on the patient-specific situation; both the patient-specific stiffness and scoliotic shape will influence this outcome, but in general the applied torsion correction moment will also improve the deformity in the frontal plane. Although some correction is found, it might be necessary to address the lateral deformity by means of another implant. In future work, also the effects of an implant correcting the lateral curve with small forces and using the adaptation and visco-elastic behaviour of soft tissues will be analysed.

In conclusion, this study showed that the currently used animal experiments to test the effectiveness of scoliosis correction implants is not a valid method, since scoliosis induction in a healthy spine is not a good model for scoliosis correction in a scoliotic spine.

\section{Acknowledgement}

This study was financially supported by the Dutch Technology Foundation, STW, applied science division of NWO and the Technology Program of the Ministry of Economic Affairs.

We thank ESI Group for the availability of the 5th percentile female model, for the use of their software (PAM-CRASH) and support. 


\section{References}

[1] T. Morais, M. Bernier, F. Turcotte, 'Age- and sex-specific prevalence of scoliosis and the value of school screening programs'. Am J Public Health Vol. 75, pp. 1377-1380, 1985.

[2] E. Rogala, D. Drummond, J. Gurr, 'Scoliosis: incidence and natural history. A prospective epidemiological study'. J Bone Joint Surg Vol. 60-A, pp. 173-176, 1978.

[3] A.J. Stirling, D. Howel, P.A. Millner, S. Sadiq, D. Sharples, R.A. Dickson, 'Late-onset idiopathic scoliosis in children six to fourteen years old. A cross-sectional prevalence study'. J Bone Joint Surg Vol. 78-A, pp. 1330-1336, 1996.

[4] H.-K. Wong, J.H.P. Hui, U. Rajan, H.-P. Chia, 'Idiopathic scoliosis in singapore schoolchildren: A prevalence study 15 years into the screening program.'. Spine Vol. 30, pp. 1188-1196, 2005.

[5] S.L. Weinstein, L.A. Dolan, J.C.Y. Cheng, A. Danielsson, J.A. Morcuende, 'Adolescent idiopathic scoliosis'. Lancet Vol. 371, pp. 1527-1537, 2008.

[6] H.-R. Weiss, S. Bess, M. Wong, V. Patel, D. Goodall, E. Burger, 'Adolescent idiopathic scoliosis - to operate or not? A debate article'. Patient Safety in Surgery Vol. 2, pp. 25-38., 2008.

[7] B.V. Reamy, J.B. Slakey, 'Adolescent Idiopathic scoliosis: review and current concepts'. Am Fam Physician Vol. 64, pp. 111-116, 2001.

[8] T. Maruyama, 'Bracing adolescent idiopathic scoliosis: A systematic review of the literature of effective conservative treatment looking for end results 5 years after weaning'. Disability \& Rehabilitation Vol. 30, pp. 786-791, 2008.

[9] P.D. Sponseller, 'Bracing for adolescent idiopathic scoliosis in practice today'. Journal of pediatric orthopaedics Vol. 31, pp. S53-S60, 2011.

[10] D. Wever, J. Elstrodt, A. Veldhuizen, J.R.v. Horn, 'Scoliosis correction with shape-memory metal: results of an experimental study'. European Spine Journal Vol. 11, pp. 100-106, 2002.

[11] P.O. Newton, C.L. Farnsworth, V.V. Upasani, R. Chambers, S.H. Yoon, P. Firkins, 'Dual and single memory rod construct comparison in an animal study'. Spine Vol. 36, pp. E904-E913. [12] W.J. Kim, S.H. Lee, S.W. Shin, C.H. Rivard, C. Coillard, S. Rhalmi, 'The influence of fixation rigidity on intervertebral joints. An experimental comparison between a rigid and a flexible system.'. J Korean Neurosurg Soc Vol. 37, pp. 364-369, 2005.

[13] I. Busscher, A.J. van der Veen, J.H. van Dieen, I. Kingma, G.J. Verkerke, A.G. Veldhuizen, 'In vitro biomechanical characteristics of the spine: A comparison between human and porcine spinal segments'. Spine Vol. 35, pp. E35-E42, 2010.

[14] H.J. Wilke, A. Kettler, K.H. Wenger, L. Claes, 'Are sheep spines a valid biomechanical model for a human spines?'. Spine Vol. 22, pp. 2365-2374., 1997.

[15] T.H. Smit, 'The use of a quadruped as an in vivo model for the study of the spine biomechanical considerations'. European Spine Journal Vol. 11, pp. 137-144., 2002.

[16] V.R. Yingling, S.M. McGill, 'Mechanical properties and failure mechanics of the spine under posterior shear load: observations from a porcine model.'. Journal of Spinal Disorders Vol. 12 , pp. 501-508, 1999.

[17] J. Carrier, C. Aubin, I. Villemure, H. Labelle, 'Biomechanical modelling of growth modulation following rib shortening or lengthening in adolescent idiopathic scoliosis'. Medical and Biological Engineering and Computing Vol. 42, pp. 541-548, 2004.

[18] G. Desroches, C.-E. Aubin, D. Sucato, C.-H. Rivard, 'Simulation of an anterior spine instrumentation in adolescent idiopathic scoliosis using a flexible multi-body model'. Medical and Biological Engineering and Computing Vol. 45, pp. 759-768, 2007.

[19] Y. Lafon, J.P. Steib, W. Skalli, 'Intraoperative three dimensional correction during in situ contouring surgery by using a numerical model'. Spine Vol. 35, pp. 453-459, 2010.

[20] Y. Majdouline, C.-E. Aubin, A. Sangole, H. Labelle, 'Computer simulation for the optimization of instrumentation strategies in adolescent idiopathic scoliosis'. Medical and Biological Engineering and Computing Vol. 47, pp. 1143-1154, 2009.

[21] J.E. Lonstein, J.M. Carlson, 'The prediction of curve progression in untreated idiopathic scoliosis during growth.'. J Bone Joint Surg Vol. 66-A, pp. 1061-1071., 1984. 
[22] S.L. Weinstein, L.A. Dolan, K.F. Spratt, K.K. Peterson, M.J. Spoonamore, I.V. Ponseti, 'Health and function of patients with untreated idiopathic scoliosis: A 50-year natural history study'. JAMA Vol. 289, pp. 559-567, 2003.

[23] D.J. Wever, A.G. Veldhuizen, J.P. Klein, P.J. Webb, G. Nijenbanning, J.C. Cool, J.R. van Horn, 'A biomechanical analysis of the vertebral and rib deformities in structural scoliosis'. European Spine Journal Vol. 8, pp. 252-260, 1999.

[24] G.J.M. Meijer, J. Homminga, E.E.G. Hekman, A.G. Veldhuizen, G.J. Verkerke, 'The effect of three-dimensional geometrical changes during adolescent growth on the biomechanics of a spinal motion segment'. J Biomech Vol. 43, pp. 1590-1597, 2010.

[25] G.J.M. Meijer, J. Homminga, A.G. Veldhuizen, G.J. Verkerke, 'Influence of interpersonal geometrical variation on spinal motion segment stiffness: Implications for patient-specific modeling'. Spine Vol. 36, pp. E929-E935 2011.

[26] G.J.M. Meijer, J. Homminga, G.J. Verkerke, 'Influence of costovertebral joints on the stiffness of the spine'. submitted.

[27] J. Cruickshank, M. Koike, R. Dickson, 'Curve patterns in idiopathic scoliosis. A clinical and radiographic study'. J Bone Joint Surg Vol. 71-B, pp. 259-263, 1989.

[28] I. Villemure, C.-E. Aubin, G. Grimard, J. Dansereau, H. Labelle, 'Progression of vertebral and spinal three-dimensional deformitites in adolescent idiopathic scoliosis. A longitudinal study.'. Spine Vol. 26, pp. 2244-2250., 2001.

[29] T.A.L. Wren, G.S. Beaupre, D.R. Carter, 'A model for loading-dependent growth, development, and adaptation of tendons and ligaments'. J Biomech Vol. 31, pp. 107-114., 1998. [30] R.E. Will, I.A. Stokes, X. Qiu, M.R. Walker, J.O. Sanders, 'Cobb angle progression in adolescent scoliosis begins at the intervertebral disc'. Spine Vol. 34, pp. 2782-2786, 2009.

[31] M.M. Sanders A memory metal based scoliosis correction system. PhD Thesis, University of Twente/ 1993.

[32] A.L.F. Nachemson, G. Elfstrom, 'Intravital wireless telemetry of axial forces in Harrington distraction rods in patients with idiopathic scoliosis'. J Bone Joint Surg Vol. 53-A, pp. 445-465, 1971.

[33] T.R. Waugh, 'Intravital measurements during instrumental correction of idiopathic scoliosis'. Acta Orthop Scand Vol. S93, pp. 1-87, 1966.

[34] N. Farahpour Influence of adolescent idiopathic scoliosis on three-dimensional kinematic of the trunk in lateral bending motion. PhD Thesis, Université de Montréal/ 1996.

[35] A.G. Veldhuizen, P.J. Scholten, 'Kinematics of the scoliotic spine as related to the normal spine.'. Spine Vol. 12, pp. 852-858, 1987.

[36] M.J. Beuerlein, V.J. Raso, D.L. Hill, M.J. Moreau, J.K. Mahood, 'Changes in alignment of the scoliotic spine in response to lateral bending.'. Spine Vol. 28, pp. 693-698, 2003

[37] I.H. Drespe, G.K. Polzhofer, A.S. Turner, J.N. Grauer, 'Animal models for spinal fusion'. The Spine Journal Vol. 5, pp. S209, 2005.

[38] J.T. Braun, E. Akyuz, J.W. Ogilvie, 'The use of animal models in fusionless scoliosis investigations'. Spine Vol. 30, pp. S35-S45 2005.

[39] F. Schwab, A. Patel, V. Lafage, J.-P. Farcy, 'A porcine model for progressive thoracic scoliosis'. Spine Vol. 34, pp. E397-E404, 2009. 


\section{Chapter 8}

General discussion 
The main goal of this thesis was to present a numerical model of an individual adolescent spine that quantifies the various biomechanical aspects that are important in scoliosis correction.

In this general discussion the methods and results from this thesis are analysed and their implications for future research are discussed.

\subsection{Discussion of the model}

The final trunk model is developed and validated in steps: from motion segment, multi-segments, total thoracolumbar spine, thoracolumbar spine including ribcage and finally the trunk model. The validation of the various steps of the model ensures the correct contribution of the various parts to the global behaviour of the trunk. The final model describes the biomechanical behaviour of the thoracolumbar spine, ribcage and intra-abdominal pressure for an average ten-year-old female. In this section, the strengths and added value of this model are compared to currently available models, the limitations for the use and interpretation of the results are discussed and finally recommendations for improvement of the model are given.

\subsubsection{Model strengths and added value}

The final model describes the passive, elastic behaviour of the spine, ribcage, intraabdominal pressure and abdominal muscles for a ten-year-old. The model has been extensively validated for various regions and loading conditions (Section 2.3).

The major strength of this model is that it describes the mechanics of the total trunk, without oversimplifying the properties of the anatomical structures like the disc, ligaments, ribs and intra-abdominal pressure. These structures still contain the detailed non-linear geometry and material properties, which can be adapted independently to a patient-specific situation.

This new model makes it possible to simulate scoliosis correction and progression, since large deformations of the intervertebral discs are taken into account and at the same time the total thoracolumbar spine can be analysed. Other applications for the model are analyses of the influence of global sagittal alignment changes (for example due to vertebral fracture or surgical correction) on the disc loading profile and/or muscle forces.

To analyse the sensitivity of various geometrical aspects on the biomechanics, a generic model of the spine is made, which can be used to easily define various geometries of the spine. Biomechanical questions, such as the influence of adolescent growth on the stiffness of the spine (Chapter 3) and the effect of patientspecific geometry on the stiffness of the spine (Chapter 4) can, and have been, answered with these models.

In this thesis, the first model of the intra-abdominal pressure (IAP) is presented, that describes the direct effect of the IAP on the vertebrae, as well as the upward force of the diaphragm on the ribcage and the (passive) stiffness of the abdominal muscles (Chapter 6). This way of modelling is very complete and can be used to analyse the various existing theories on the working mechanism of the intra-abdominal pressure. 


\subsubsection{Model limitations}

A major limitation of the model is that it only describes the instantaneous elastic behaviour of the spine, and the behaviour under sustained loading cannot be analysed. It is well-know that especially the ligaments and intervertebral disc mechanical properties are highly dependent on loading rate and loading history. The poro-elastic properties of the intervertebral disc will result in a flow of the water away from areas with high stress, increasing its stiffness with sustained loading. Also viscoelastic properties of the annulus fibres and ligaments will result in an increasing stiffness with sustained loading, although creep will also result in higher deformations with sustained loading. In the model these aspects are not considered, which is partly due to the used solving method, but mostly due to the lack of validation data and material properties considering the behaviour of the spine under sustained loading. Although some creep experiments do exist for the intervertebral disc ${ }^{1-3}$, motion segments $^{1,4,5}$ and even multi-segments ${ }^{6,7}$, implementation of the results are difficult. Magnitude and direction of the loading, rate of the load-change and time-dependent behaviour need to be considered. None of the experiments considers all of these aspects, since creep-experiments are time-expensive and the quality of the specimen will degrade with testing-time. Comparison between the various tests is impossible due to the variance in loading conditions, tested subjects (inter-personal differences are large, and age-related effects are very important ${ }^{7}$ ) and test set-up. Most experiments consider axial compression loading, while our main interest lies in lateral bending and axial rotation and the results are expected to be very different.

The second limitation of the model lies in the validity of the stress profiles of the disc, which is limited due to a number of reasons. First of all, the vertebrae are modelled as rigid bodies, neglecting the deformity of the cartilage endplates, which can be considerable in compression ${ }^{8}$ and under larger bending moments ${ }^{9}$. Secondly, the variation of fibre volume fraction and stiffness for different locations in the disc (as described by Shirazi-Adl ${ }^{10}$ ) is not modelled. This is expected to have an essential influence on the stress pattern within the intervertebral disc. When assuming a homogeneous distribution of the stiffness, due to bulging of the nucleus the inner fibres are more strained under axial compression than the outer ones, and this will result in an overestimation of the stress in the centre of the disc compared to the outer part of the disc.

Thirdly, the pretension in the annulus fibres is neglected, resulting in an underestimation of the hydrostatic pressure in the disc, although a similar effect (but smaller) is achieved by applying pretension in the spinal ligaments. The stress profiles of the disc can therefore not be used in quantitative analyses, but only in qualitative analyses. In this thesis, the stress profiles of the disc are therefore not used.

One of the major simplifications of the motion segment model is the representation of the facet joints as parallel, planar surfaces, instead of a geometry that is curved in $3 \mathrm{D}$, as it is in reality. Simulations into the effects of the geometry of the facet joints of the L3-L4 motion segment showed that representation by a 3D curved surface instead of planar surfaces did result in earlier contact between the facet surfaces, but due to the non-linear contact-definition, the effects on the load-displacement curve are small. 
A similar simplification has been made for the contacts between the ribs and the vertebrae. Due to a different type of contact (penalty contact rather than describing force-penetration curve), a combination with a plane curved in 3D was not possible. It is expected that the effects on the mechanics of the vertebrae-rib contact of nonlinearity in the contact definitions are more important than the 3D curvature of the planes. Since the mechanical behaviour of the isolated rib joints are validated, this assumption appears acceptable.

The elastic material properties of the soft tissues are also a simplification, because the actual properties, including non-linear and non-homogenous behaviour, are often unknown. Most important and limiting is the large variety between subjects, which makes an estimation of the average material properties difficult. One of the main assumptions for the material properties is the linear behaviour of the annulus fibres and neglecting the variation of fibre volume fraction and stiffness for different locations in the disc. Smit ${ }^{11}$ pointed out that non-linear stress-strain relationship for the annulus fibres is unnecessary when analysing physiological conditions, since the fibres are only strained in the linear range under physiological loading conditions and kinematic behaviour of the disc with linear fibres is realistic for all loading cases. Neglecting the variation of fibre volume fraction and stiffness for different locations in the disc mainly influences the stress profile, but the influence on the loaddisplacement relations is negligible ${ }^{12}$.

Validation of the adolescent model is limited, due to lack of adolescent in vitro data. For the same reason, material properties of adolescents are also unknown, and available (young) adult data had to be used. Simulations with the adolescent model are consistent with available literature, but true validation is not possible. This limitation also stresses the importance of a model representative for the adolescent spine and trunk: in vitro testing is almost impossible in young humans.

\subsubsection{Recommendations for improvement of model}

Neither material properties nor kinematic data are available for adolescents. More kinematic data would improve the validation of adolescent models and improve the basic biomechanical knowledge. As an alternative, adolescent in vivo experiments could be used. The total trunk model could be validated with these data. Current in vivo experimental data are insufficient, since loading conditions are unknown (often only the voluntary range of motion is reported) and measurements are inaccurate and limited (the movement of the spine is measured by applying markers at the skin, above a number of vertebrae). When in vivo experiments are conducted, it is therefore very important to be able to control and measure the actual loading, as well as the movement, of the spine and trunk.

Improvement of the prediction of the long-term outcome of scoliosis correction could be achieved by applying viscoelastic behaviour and stress-adaptation for the intervertebral disc and ligaments. For this, more detailed data is necessary, especially for behaviour under sustained lateral bending and axial rotation loading. Although some validation data for multi-segments under lateral bending and axial rotation loading exists ${ }^{6}$, data on material and/or motion segment level would make 
the number of parameters that can be fine-tuned in the validation process more organized.

Before the stress profiles of the disc can be used with confidence in a quantitative way, an inhomogeneous representation of the fibre thickness and stiffness throughout the disc needs to be modelled and pretension in these fibres is necessary to simulate the osmotic pressure in the disc. Also a more thorough validation of the disc pressure profiles with available literature is recommended.

\subsection{Discussion of the results}

In this section the results of the chapters 3 to 7 and their (clinical) implications are discussed. First, the results from the studies carried out with motion segment models need an extrapolation to the effects on the total trunk mechanics. This is done for the effects of the both the patient-specific geometry and adolescent growth.

The results from the various studies are used to optimize the development and evaluate the design of a scoliosis correction implant, but also to improve the knowledge about modelling of scoliosis correction and to increase the basic knowledge about the biomechanics of the spine and trunk. The implications of the results for these three aspects are discussed separately. Finally, the future directions of scoliosis correction modelling are discussed.

\subsubsection{Effect of patient-specific geometry of the total spine and of the trunk on biomechanics}

In chapter 4 the effects of the patient-specific parameters on the biomechanics of the L3-L4 motion segment are described. For application in scoliosis research, the results actually need extrapolation to the mechanical behaviour of the total spine and trunk in scoliotic subjects. Of course no quantitative conclusions can be drawn this far, but some qualitative aspects can be considered.

First of all, it is expected that for the total spine the patient-specific disc height is also the most important geometric parameter that needs accurate patient-specific determination. Furthermore, it is expected that the patient-specific lordosis and kyphosis will have a larger effect on the behaviour of the total thoracolumbar spine, since the changes for the total spine are larger than for one motion segment. This theory has been proven by simulations where the effects of a changing lordosis with a multi-segment (L1-L4) were studied and a reduction of the lordosis by 10 degrees, resulted into a reduction of the stiffness of up to $50 \%$ in flexion and extension.

Secondly, it is pointed out that for scoliotic spines, it is expected that also the wedging of the disc in the frontal plane has a large influence on the mechanical behaviour of the spine and therefore needs accurate determination.

For the geometry of the total trunk, the circumferential size of the ribcage and trunk (both depth and width) have a large influence on especially axial rotation stiffness, which is of major concern in scoliosis correction. These parameters therefore need patient-specific determination. 


\subsubsection{Effect of growth of the total spine and of the trunk on biomechanics}

In chapter 3 the effects of three-dimensional adolescent growth on the biomechanics of the L3-L4 motion segment are described. For application in scoliosis research, the results actually need extrapolation to the mechanical behaviour of the total spine and trunk in scoliotic subjects. Of course no quantitative conclusions can be drawn this far, but some qualitative aspects can be considered.

First of all, for the total spine no significant difference for the influence of most of the analysed parameters is expected, except for the lordosis and kyphosis angle. As pointed out in the previous section, the change in the total spine is larger than for one segment, and larger effects on the stiffness, especially in flexion and extension, have been found with a pilot study.

Secondly, growth of the ribcage is mainly length growth of the ribs, which affects the total circumferential size of the ribcage, but the shape of the ribcage is not influenced $^{13}$. The rib-vertebral-angle also does not change during adolescence ${ }^{13}$. It is therefore expected that the overall effect of the ribcage does not change during adolescence. But as pointed out in the discussion of chapter 6 , it is unclear whether the relative contribution of the spine, ribcage and intra-abdominal pressure to the mechanics of the total trunk change during adolescence.

Finally, it is assumed that growth of the adolescent scoliotic spine is comparable to that of a healthy spine. Some studies did analyse the difference between growth in healthy and scoliotic subjects. Veldhuizen et $\mathrm{al}^{14}$ compared width and height growth for the vertebrae within and outside the scoliotic curve of the same girls and no significant difference was found. Only one study analyses the three-dimensional growth effects during idiopathic scoliosis ${ }^{15}$. In this study the width, length and depth of the trunk of adolescents with idiopathic scoliosis were compared to those of a healthy control group and no significant differences were found. All together it can be concluded that the total amount of growth during adolescent growth is comparable with healthy girls, although girls with idiopathic scoliosis might have an earlier growth spurt than healthy girls ${ }^{16}$.

The scoliotic curve may influence the growth of the vertebrae, by the changing loading pattern of the vertebrae and the stress-dependent component of growth (Hueter-Volkmann law). This is of main interest when modelling scoliosis progression and less essential when correction of mild scoliosis is analysed, since bone deformation occurs in later, and more severe, stages of scoliosis.

\subsubsection{Implications of obtained results for development of scoliosis correction implant}

First of all, although the short term correction of the new scoliosis correction implant is small, the long-term correction is considerable (Chapter 7), especially when we consider that only two iteration steps were simulated (rest moment is $1.4 \mathrm{Nm}$ and will go to $0 \mathrm{Nm}$ in reality). A long term correction of a mild scoliosis with relative low moments (1.5 Nm torsion) thus seems realistic. It is however unclear, which time scale is related to this process. 
We also showed that a torsion implant can correct the Cobb angle of scoliotic patients (Chapter 7). The ratio between these corrections of course depends on the patient-specific situation; both the patient-specific stiffness and scoliotic shape will influence this outcome, but in general the applied torsion correction moment will also improve the deformity in the frontal plane. Although some correction is found, it might be necessary to address the lateral deformity by means of another implant to gain full correction.

Secondly, the implant should compensate for the increasing stiffness of the spine during adolescent growth. When the implant is used between 10 year and maturity, the stiffness of the spine will increases $40 \%$ due to the changing geometry (Chapter 3). When the design of the implant does not compensate for this effect, the scoliosis correction will be less over time, due to the increasing stiffness of the spine and a complete correction might not be reached.

Comparison of scoliosis correction and induction in our models showed that the mechanical effects of a torsion loading are not similar (Chapter 7). Although both showed an effect in the rotation angle and lateral plane, and no effects on the kyphosis, the effects in the lateral plane were much higher in the scoliosis correction simulations than in the scoliosis induction simulations. This suggests a different relation between the applied loading and coupled movements for scoliotic and healthy spines.

An alternative for these animal experiments, however, is hard to find. To improve the usability of the results of the animal experiments, it might be better to first induce scoliosis in the animals. On the other hand, ethical reasons, but also the questions about the true underlying effects of scoliosis and how to model these, will hamper these experiments as well.

\subsubsection{Implications of obtained results for modelling of scoliosis correction}

While length growth shows the largest geometrical increase and is currently the only aspect that is modelled, the largest mechanical effects are seen for the width and depth increase of the spine (Chapter 3). The mechanical effect of the total geometrical growth of the spine on the stiffness of spine is large (40\%) and neglecting this effect will result in an underestimation of the stiffness of the spine and overestimation of the scoliosis correction.

Modelling of a patient-specific geometry is necessary to predict a patient-specific stiffness and the accordingly expected patient-specific scoliosis correction. The largest influence has been found for the disc height (Chapter 4), but also the wedging of the discs in scoliosis is of main importance. For a good prediction of the patientspecific scoliosis correction outcome, in vivo stiffness measurements would be most desirable, but current methods are insufficient due to unknown loading conditions.

For a good estimation of the in vivo stiffness of the trunk of a scoliosis patient, modelling of the ribcage and intra-abdominal pressure are essential (Chapter 6). 


\subsubsection{Implications of obtained results for basic knowledge on biomechanics of the spine}

Interpersonal geometry variation contribute to a large extend to the interpersonal variation in stiffness. Especially the disc height is important and can contribute to a variance of $300 \%$ in the stiffness of a motion segment (Chapter 4).

In in vitro tests often the posterior part of the ribs $(3 \mathrm{~cm})$ is left attached for practical reasons, assuming a negligible influence on the mechanics of the spine. A comparison between models including and excluding these posterior part of the ribs, shows an increase in stiffness for lateral bending of the spine for multi-segments (Chapter 5) and an increase of the stiffness in all motion directions for the total thoracic spine (unpublished).

The effect of the thoracolumbar spine, ribcage and IAP on the stiffness of the spine is quantified. Although the used model is representative for a ten-year-old, similar results are expected for adult trunks (Chapter 6).

\subsubsection{Future directions in scoliosis correction modelling}

The current scoliotic model describes an average scoliosis and not a specific patient. For clinical use, a patient-specific outcome is wanted. This does not only require patient-specific geometry, but also (an estimation of) patient-specific material properties. In vivo stiffness measurements would therefore be most desirable, but current methods are insufficient due to unknown loading conditions.

Implementation of growth into the model is especially important for questions relating to scoliosis progression. It is expected that long term correction of mild scoliosis is mainly due to adaptation of soft tissues, and less influence is expected from growth modulation, since the remodelling of bone is taking more time than the remodelling of the soft tissues.

It should however be taken into account that 3D growth of the spine will increase the stiffness of the spine, and thereby influence the outcome of scoliosis correction.

Implementation of viscoelastic behaviour and adaptation of soft tissues (disc and ligaments) are necessary to predict the long term scoliosis correction. For a good implementation, more data on viscoelastic properties and adaptation of the various tissues are needed. 


\subsection{Concluding remarks}

In the current thesis, a numerical model of an average adolescent trunk is developed. The biomechanical behaviour of the developed model of the adolescent spine, ribcage and trunk were compared to literature and show similar behaviour, although validation of the adolescent models and scoliotic models are difficult due to lack of in vitro and in vivo data. No studies into the mechanical properties on material level, spinal level or trunk level have been published for the adolescent or scoliotic spine. Furthermore, since the true cause of idiopathic scoliosis is unknown, it is difficult to model the mechanical effects of scoliosis (other than the changing geometry) compared to the healthy spine. However, results from the current model are not inconsistent with current knowledge concerning the biomechanics of the spine and trunk, the differences in the biomechanics of the spine between adults and adolescents, and the mechanics of the scoliotic spine.

It is therefore believed that the model is representative for the general, passive behaviour of the adolescent healthy and scoliotic spine/trunk and can be used to predict scoliosis correction and answer biomechanical questions related to scoliosis research.

The various biomechanical aspects that are important in scoliosis correction were quantified: the influence of adolescent growth and inter-personal variations in geometry on the stiffness of the spine were analysed, but also the relative contributions of the thoracolumbar spine, ribcage and intra-abdominal pressure to the biomechanics of the trunk were analysed. The short-term outcome of the new scoliosis correction implant was estimated. Both the axial rotation and lateral deformity were analysed, for an implant that applies a torsion moment. Simulations proof that the correction is improving over time, due to adaptation and viscoelastic behaviour of the soft tissues. However, data on the timescale of these processes are lacking, making an estimation of the duration of the correction not possible.

In the future, the long-term prediction of scoliosis correction by numerical modelling might be improved by addition of viscoelastic behaviour and implementation of growth aspects. For a good clinical tool, however, patient-specific modelling, in which especially the disc height needs an accurate determination, seems the most logical first improvement. 


\section{References}

[1] M. Kurutz, 'In vivo age- and sex-related creep of human lumbar motion segments and discs in pure centric tension'. J Biomech Vol. 39, pp. 1180-1190., 2006.

[2] W. Koeller, W. Meier, F. Hartmann, 'Biomechanical properties of human intervertebral discs subjected to axial dynamic compression: a comparison of lumbar and thoracic discs'. Spine Vol. 9, pp. 725-733, 1984.

[3] J.C. Iatridis, M. Weidenbaum, L.A. Setton, V.C. Mow, 'Is the nucleus pulposus a solid or a fluid? Mechanical behaviors of the nucleus pulposus of the human intervertebral disc.'. Spine Vol. 21, pp. 1174-1184., 1996.

[4] M.A. Adams, P. Dolan, 'Time dependent changes in the lumbar spine's resitance to bending.'. Clin Biomech Vol. 11, pp. 194-200., 1996.

[5] T.S. Keller, D.M. Spengler, T.H. Hansson, 'Mechanical behavior of the human lumbar spine. I. Creep analysis during static compressive loading'. Journal of Orthopaedic Research Vol. 5, pp. 467-478, 1987.

[6] I. Busscher, J.H. van Dieën, A.J. van der Veen, I. Kingma, G.J.M. Meijer, G.J. Verkerke, A.G. Veldhuizen, 'The effects of creep and recovery on the in vitro biomechanical characteristics of human multi-level thoracolumbar spinal segments'. Clinical Biomechanics Vol. 26, pp. 438-444, 2011.

[7] L. Twomey, J. Taylor, 'Flexion creep deformation and hysteresis in the lumbar vertebral column.'. Spine Vol. 7, pp. 116-122, 1982.

[8] J.J. MacLean, J.P. Owen, J.C. latridis, 'Role of endplates in contributing to compression behaviors of motion segments and intervertebral discs'. J Biomech Vol. 40, pp. 55-63, 2007.

[9] A. Shirazi-Adl, 'Analysis of role of bone compliance on mechanics of a lumbar motion segment'. J Biomech Eng Vol. 116, pp. 408-412., 1994.

[10] A. Shirazi-Adl, S.C. Shrivastava, A.M. Ahmad, 'Stress analysis of the lumbar disc-body unit in compression. A three-dimensional nonlinear finite element study.'. Spine Vol. 9, pp. 120-134., 1984.

[11] T.H. Smit The mechnical significance of the trabecular bone architecture in a human vertebra. PhD Thesis, Technischen Universitaet Hanburg-Harburg/ 1996.

[12] A. Shirazi-Adl, 'On the fibre composite material models of disc annulus - comparison of predicted stresses.'. J Biomech Vol. 22, pp. 357-365, 1989.

[13] P. Openshaw, S. Edwards, P. Helms, 'Changes in rib cage geometry during childhood'. Thorax Vol. 39, pp. 624-627, 1984.

[14] A.G. Veldhuizen, P. Baas, P.J. Webb, 'Observations on the growth of the adolescent spine'. J Bone Joint Surg Vol. 68-B, pp. 724-728, 1986.

[15] Y.P. Charles, A. Diméglio, M. Marcoul, J.-F. Bourgin, A. Marcoul, M.-C. Bozonnat, 'Influence of idiopathic scoliosis on three-dimensional thoracic growth'. Spine Vol. 33, pp. 12091218., 2008.

[16] G. Hagglund, J. Karlberg, S. Willner, 'Growth in girls with adolescent idiopathic scoliosis'. Spine Vol. 17, pp. 108-111., 1992. 


\section{Appendices}
A Quantitative data per vertebral level
B Definition of planes and local coordinate system
C Shape of the endplates 


\section{A Quantitative data per vertebral level}

For each of the vertebral levels, the dimensions of the vertebral body, the disc and the vertebral arch, and the facet surfaces are given in table 1, 2 and 3 respectively.

\begin{tabular}{|c|c|c|c|c|c|c|}
\hline & $\begin{array}{c}\text { inferior } \\
\text { endplate } \\
\text { depth }[\mathrm{mm}]\end{array}$ & $\begin{array}{c}\text { superior } \\
\text { endplate } \\
\text { depth [mm] }\end{array}$ & $\begin{array}{c}\text { inferior } \\
\text { endplate } \\
\text { width [mm] }\end{array}$ & $\begin{array}{c}\text { superior } \\
\text { endplate } \\
\text { width [mm] }\end{array}$ & $\begin{array}{c}\text { vertebral } \\
\text { body height } \\
\text { [mm] }\end{array}$ & $\begin{array}{c}\text { vertebra } \\
\text { lordosis angle } \\
{\left[{ }^{\circ}\right]}\end{array}$ \\
\hline T1 & 19.7 & 18.5 & 27.8 & 24.5 & 14.1 & -5.7 \\
\hline $\mathbf{T} 2$ & 21.6 & 19.6 & 27.4 & 24.9 & 15.6 & -3.6 \\
\hline T3 & 23.3 & 22.7 & 25.9 & 24.6 & 15.7 & -4.5 \\
\hline T4 & 24.5 & 23.3 & 26.0 & 24.5 & 16.2 & -3.8 \\
\hline T5 & 25.8 & 24.3 & 27.0 & 24.9 & 16.2 & -4.1 \\
\hline T6 & 26.9 & 26.0 & 28.2 & 26.2 & 17.4 & -3.9 \\
\hline T7 & 28.5 & 27.4 & 29.1 & 27.8 & 18.2 & -3.8 \\
\hline T8 & 29.4 & 27.9 & 30.5 & 29.5 & 18.7 & -3.6 \\
\hline T9 & 31.0 & 29.3 & 33.0 & 30.6 & 19.3 & -2.1 \\
\hline T10 & 31.6 & 30.5 & 35.4 & 31.9 & 20.2 & -1.7 \\
\hline T11 & 31.8 & 31.9 & 39.1 & 34.9 & 21.3 & -4.5 \\
\hline T12 & 33.4 & 32.8 & 42.1 & 39.0 & 22.7 & 4.0 \\
\hline L1 & 35.3 & 34.1 & 43.3 & 41.2 & 23.8 & 6.7 \\
\hline L2 & 34.9 & 34.6 & 45.5 & 42.6 & 24.3 & 5.6 \\
\hline L3 & 34.8 & 35.2 & 48.0 & 44.1 & 23.8 & 4.4 \\
\hline L4 & 33.9 & 35.5 & 49.5 & 46.6 & 24.1 & 7.4 \\
\hline L5 & 33.2 & 34.7 & 49.4 & 47.3 & 22.9 & 4.0 \\
\hline Ref. & 1,2 & 1,2 & 1,2 & 1,2 & 1,2 & 1,2 \\
\hline
\end{tabular}

Table 1: Quantitative data per vertebral level for vertebral body dimension

\begin{tabular}{|c|c|c|c|c|c|c|c|}
\hline & $\begin{array}{c}\text { disc } \\
\text { height } \\
{[\mathrm{mm}]}\end{array}$ & $\begin{array}{c}\text { disc } \\
\text { lordosis } \\
\text { angle }\left[{ }^{\circ}\right]\end{array}$ & $\begin{array}{c}\text { transverse } \\
\text { process } \\
\text { width }[\mathrm{mm}]\end{array}$ & $\begin{array}{c}\text { spinous } \\
\text { process } \\
\text { length [mm] }\end{array}$ & $\begin{array}{l}\text { spinous } \\
\text { process } \\
\text { angle }\left[{ }^{\circ}\right]\end{array}$ & $\begin{array}{c}\text { spinal } \\
\text { canal depth } \\
{[\mathrm{mm}]}\end{array}$ & $\begin{array}{c}\text { spinal } \\
\text { canal width } \\
{[\mathrm{mm}]}\end{array}$ \\
\hline T1 & 4.9 & $-0.5 *$ & 75.3 & $65.0 *$ & $25.0 *$ & 15.3 & 21.8 \\
\hline $\mathbf{T 2}$ & 4.9 & $-1.0 *$ & 69.4 & $60.0 *$ & $27.0 *$ & 15.3 & 19.5 \\
\hline $\mathbf{T 3}$ & 5.5 & $-1.0 *$ & 60.8 & $52.0 *$ & $37.0 *$ & 16.2 & 18.3 \\
\hline $\mathbf{T 4}$ & 6.0 & $-1.5 *$ & 56.9 & $53.0 *$ & $45.0 *$ & 16.2 & 17.0 \\
\hline T5 & 6.1 & $-1.5 *$ & 61.1 & $60.0 *$ & $53.0 *$ & 16.3 & 17.1 \\
\hline T6 & 6.3 & $-2.0 *$ & 61.3 & $61.0 *$ & $52.0 *$ & 16.5 & 17.3 \\
\hline T7 & 6.5 & $-2.0 *$ & 60.4 & $61.0 *$ & $46.0 *$ & 16.1 & 17.3 \\
\hline T8 & 6.7 & $-2.0 *$ & 59.9 & $58.0 *$ & $45.0 *$ & 15.9 & 17.7 \\
\hline T9 & 7.7 & $-2.5 *$ & 59.3 & $55.0 *$ & $44.0 *$ & 15.7 & 17.9 \\
\hline T10 & 8.5 & $-2.5 *$ & 58.4 & $53.0 *$ & $40.0 *$ & 15.5 & 18.2 \\
\hline T11 & 9.1 & $-2.5 *$ & 52.2 & $50.0 *$ & $34.0 *$ & 16.0 & 19.4 \\
\hline T12 & 6.5 & 5.0 & 46.9 & $48.0 *$ & $25.0 *$ & 18.1 & 22.2 \\
\hline L1 & 7.5 & 5.5 & 71.2 & 47.0 & 22.0 & 19.0 & 23.7 \\
\hline L2 & 9.0 & 6.0 & 76.1 & 50.0 & 24.0 & 18.2 & 23.8 \\
\hline L3 & 10.0 & 7.0 & 85.7 & 50.6 & 25.0 & 17.5 & 24.3 \\
\hline L4 & 9.0 & 9.0 & 79.4 & 48.4 & 28.0 & 18.6 & 25.4 \\
\hline L5 & & & 92.5 & 43.9 & 34.0 & 19.7 & 27.1 \\
\hline Ref. & 3,4 & 3 & 1,2 & 5 & 5 & 1,2 & 1,2 \\
\hline
\end{tabular}

Table 2: Quantitative data per vertebral level for dimensions of the disc and vertebral arch. Note that for the disc, the level below the vertebrae is indicated, so T1 means the T1-T2 disc. 


\begin{tabular}{|c|c|c|c|c|c|}
\hline & $\begin{array}{l}\text { Longitudinal } \\
\text { facet angle }\left[{ }^{\circ}\right]\end{array}$ & $\begin{array}{c}\text { transverse } \\
\text { facet angle }\left[^{\circ}\right]\end{array}$ & $\begin{array}{l}\text { inter facet } \\
\text { width [mm] }\end{array}$ & $\begin{array}{l}\text { facet width } \\
\text { [mm] }\end{array}$ & $\begin{array}{c}\text { facet height } \\
{[\mathrm{mm}]}\end{array}$ \\
\hline T1 superior & 32.9 & 99.5 & 33.2 & 13.7 & 12.6 \\
\hline $\mathrm{T} 1$ inferior & 30.4 & 108.3 & 28.2 & 12.6 & 12.9 \\
\hline T2 superior & 25.1 & 106.0 & 28.2 & 11.9 & 12.6 \\
\hline $\mathrm{T} 2$ inferior & 26.6 & 108.3 & 24.3 & 11.1 & 11.5 \\
\hline $\mathrm{T} 3$ superior & 22.6 & 106.6 & 24.6 & 10.7 & 11.3 \\
\hline T3 inferior & 22.9 & 107.7 & 22.3 & 10.2 & 10.9 \\
\hline T4 superior & 21.8 & 105.2 & 22.1 & 10.4 & 11.3 \\
\hline T4 inferior & 21.5 & 126.6 & 21.1 & 10.1 & 11.0 \\
\hline T5 superior & 20.4 & 106.0 & 20.8 & 10.2 & 11.5 \\
\hline T5 inferior & 19.6 & 108.6 & 20.8 & 9.7 & 11.1 \\
\hline T6 superior & 20.1 & 106.2 & 20.9 & 9.9 & 11.8 \\
\hline T6 inferior & 17.2 & 107.8 & 21.4 & 9.7 & 10.7 \\
\hline T7 superior & 17.9 & 105.4 & 21.0 & 9.8 & 11.5 \\
\hline T7 inferior & 15.4 & 107.6 & 22.3 & 10.1 & 10.8 \\
\hline T8 superior & 17.4 & 105.0 & 21.8 & 10.2 & 11.6 \\
\hline T8 inferior & 16.6 & 107.1 & 23.4 & 10.6 & 11.3 \\
\hline T9 superior & 16.8 & 105.3 & 23.0 & 10.9 & 11.9 \\
\hline T9 inferior & 17.2 & 107.0 & 24.6 & 12.3 & 12.4 \\
\hline T10 superior & 16.3 & 104.9 & 24.2 & 12.2 & 12.2 \\
\hline T10 inferior & 18.7 & 104.9 & 24.6 & 11.7 & 12.1 \\
\hline T11 superior & 17.8 & 103.5 & 25.2 & 11.4 & 12.3 \\
\hline T11 inferior & 20.4 & 80.5 & 24.6 & 11.2 & 12.9 \\
\hline T12 superior & 18.3 & 79.4 & 25.9 & 10.9 & 13.0 \\
\hline T12 inferior & 22.1 & 37.9 & 24.9 & 9.7 & 12.6 \\
\hline L1 superior & 10.2 & 32.1 & 26.2 & 10.4 & 12.5 \\
\hline L1 inferior & 15.2 & 25.5 & 24.8 & 11.6 & 15.5 \\
\hline L2 superior & 9.1 & 24.5 & 26.4 & 11.3 & 14.6 \\
\hline L2 inferior & 18.1 & 31.4 & 26.6 & 12.7 & 16.2 \\
\hline L3 superior & 9.8 & 29.8 & 28.6 & 13.9 & 16.0 \\
\hline L3 inferior & 22.6 & 43.4 & 29.1 & 13.6 & 16.1 \\
\hline L4 superior & 9.9 & 38.2 & 31.4 & 14.7 & 16.7 \\
\hline L4 inferior & 24.7 & 52.2 & 34.8 & 14.4 & 15.9 \\
\hline L5 superior & 10.8 & 44.8 & 35.0 & 15.6 & 17.4 \\
\hline L5 inferior & 29.3 & 53.5 & 40.6 & 15.9 & 17.9 \\
\hline Reference & 6 & 6 & 7 & 7 & 7 \\
\hline
\end{tabular}

Table 3: Quantitative data per vertebral level for facet surfaces 


\section{References}

[1] M.M. Panjabi, V. Goel, T. Oxland, K. Takata, J. Duranceau, M. Krag, M. Price, 'Human lumbar vertebrae. Quantitative three-dimensional anatomy.'. Spine Vol. 17, pp. 299-306., 1992.

[2] M.M. Panjabi, K. Takata, V. Goel, D. Federico, T. Oxland, J. Duranceau, M. Krag, 'Thoracic human vertebrae. Quantitative three-dimensional anatomy.'. Spine Vol. 16, pp. 888-901., 1991.

[3] M.F. Eijkelkamp On the development of an artificial intervertebral disc. PhD thesis, Rijksuniversiteit Groningen/ 2002.

[4] K. Chanchairujira, C.B. Chung, J.Y. Kim, O. Papakonstantinou, M.H. Lee, P. Clopton, D. Resnick, 'Intervertebral disk calcification of the spine in an elderly population: radiographic prevalence, location, and distribution and correlation with spinal degeneration'. Radiology Vol. 230, pp. 499-503, 2004.

[5] I. Gilad, M. Nissan, 'Sagittal evaluation of elemental geometrical dimensions of human vertebrae'. J anat Vol. 143, pp. 115-120., 1985.

[6] Y. Masharawi, B. Rothschild, G. Dar, S. Peleg, D. Robinson, E. Been, I. Hershkovitz, 'Facet orientation in the thorocolumbar spine. Three-dimensional anatomic and biomechanical analysis'. Spine Vol. 29, pp. 1755-1763., 2004.

[7] M.M. Panjabi, T. Oxland, K. Takata, V. Goel, J. Duranceau, M. Krag, 'Articular facets of the human spine. Quantitative three-dimensional anatomy.'. Spine Vol. 18, pp. 1298-1310., 1993. 


\section{B Definition of planes and local coordinate system}

\section{Planes of the human body}

For defining various anatomical structures and movements, three orthogonal anatomical planes are defined (figure 1).

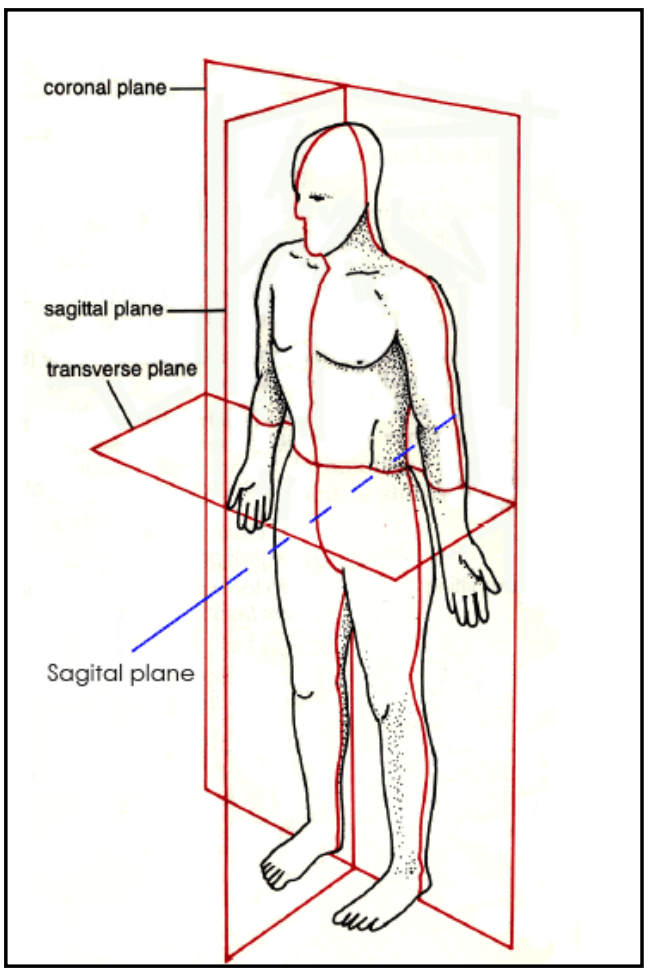

Figure 1: Definition of the planes in the human body. ${ }^{1}$

The coronal or frontal plane divides the human body in an anterior and posterior section, the transverse plane divides the human body in an upper and lower section, and the sagittal plane divides the human body in a left and right section.

\section{Local coordinate system}

To define the translational and rotational movements of a motion segment, a local coordinate system in each of the vertebrae is used (figure 2).

The movement of the upper vertebra with respect to the lower vertebra is than the movement of the motion segment. The definition of this local origin is consistent with the definition as proposed by the scoliosis research society ${ }^{2}$. 


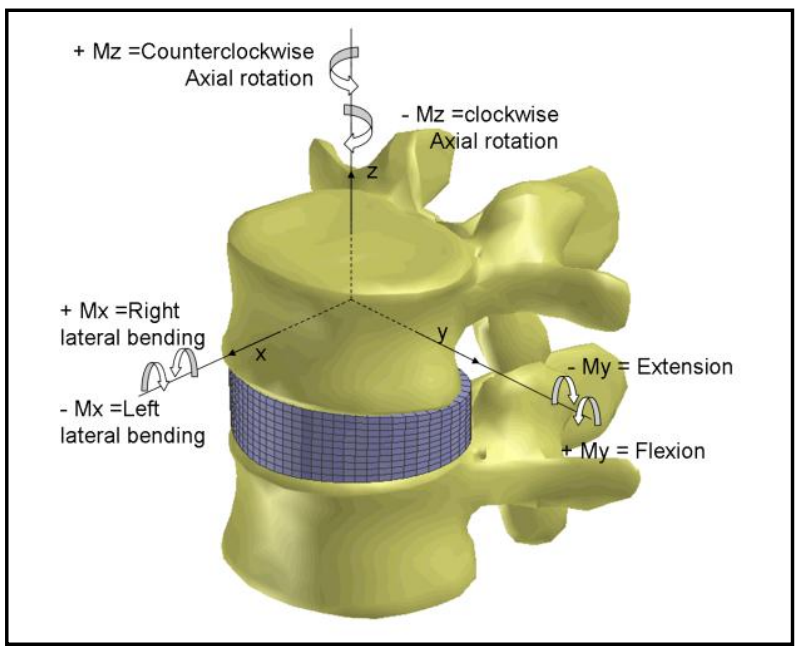

Figure 2: Definition of the local coordinate system of the upper vertebra and the motions of the motion segment

The origin of the local coordinate system is positioned in the middle of the centres of the two endplates. Three orthogonal axes through the origin are defined: the positive $x$-axis is pointing forwards, the positive $y$-axis to the left and the positive $z$-axis upwards. Translations along the positive axes are considered positive and right handed rotations around the axes are considered positive.

\section{References}

[1] P. Bruckner, K. Khan (eds) (2005) Clinical sports medicine 1st edn. McGraw-Hill, Sydney, Australia

[2] Scoliosis Research Society ' A Glossary of Scoliosis Terms.'. Spine Vol. 1, pp. 57-58., 1976 


\section{Shape of the endplates}

\section{Transverse plane}

For the shape of the endplate in the transverse plane, a general equation for a kidney shaped closed contour was defined ${ }^{1}$, by making the radial coordinate a function of the angular coordinate (polar coordinate system) (equation 1):

$$
\mathrm{r}(\theta)=\mathrm{c} 1 *[1+\mathrm{c} 2 *\{\mathrm{c} 3 * \cos (\theta)+\mathrm{c} 4 * \cos (2 \theta)+\mathrm{c} 5 * \cos (3 \theta)\}]
$$

(equation 1)

Since there are differences between the shape of the endplate for the thoracic and lumbar region (figure 1), different parameters for different regions of the spine are defined by Langrana et al. ${ }^{2}$ (table 1 ).

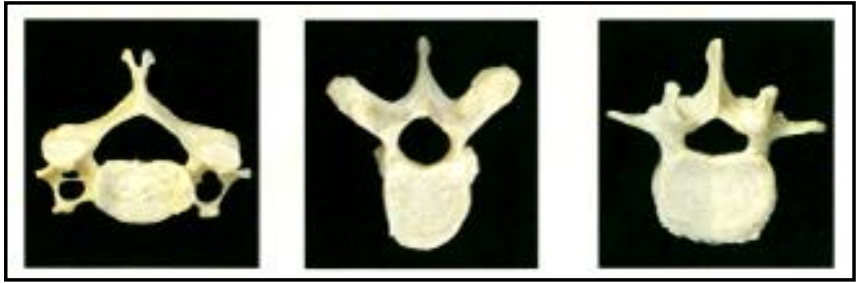

Figure 1: Shape of the cervical (left), thoracic (middle) and lumbar (right) vertebrae in the transverse plane ${ }^{3}$

\begin{tabular}{|rc|cc|}
\hline & Cervical & Thoracic & Lumbar \\
$\mathbf{c 1}$ & 12 & 8.5 & 18 \\
$\mathbf{c 2}$ & 0.075 & 0.055 & 0.075 \\
$\mathbf{c 3}$ & 1.25 & 0.6 & 1 \\
$\mathbf{c 4}$ & -3 & -2.5 & -2.5 \\
$\mathbf{c 5}$ & 1.25 & 0.9 & 1 \\
& &
\end{tabular}

Table 1: Parameters for the different endplate shapes of the spine

\section{Sagittal plane}

Especially the lumbar endplates show a relevant concavity, in the sagittal plane. This is modelled assuming an exponential decrease of this concavity(c), with increasing distance from the centre of the endplate $(r)$. The total formula is scaled for the maximum concavity $\left(c_{\max }\right)$ en the outer profile of the endplate $\left(r_{\max }\right)$. The maximum concavity for each of the endplates is different for each of the vertebral levels and is given in table 2. 


$$
\mathrm{c}(\mathrm{r})=\mathrm{c}_{\max } *\left[\frac{1}{1+\mathrm{e}^{(3-(7.7 * \mathrm{r} / \mathrm{rmax}))}}-\frac{1}{1+\mathrm{e}^{3}}\right]
$$

A visual impression (scaling is adapted for clarity reasons) of the vertebral endplate in the midsagittal plane is given in figure 2.This figure is for the profile of the lower endplate of vertebrae, the upper endplate is a mirrored version of this profile.

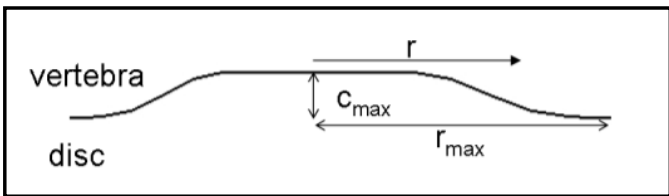

Figure 2: Profile of the lower endplate in the midsagittal plane.

\begin{tabular}{|rcc|}
\hline T1 & $\begin{array}{c}\text { superior endplate } \\
\text { concavity[mm] }\end{array}$ & $\begin{array}{c}\text { inferior endplate } \\
\text { concavity[mm] }\end{array}$ \\
T2 & $0.2 *$ & $0.2 *$ \\
T3 & $0.2 *$ & $0.2 *$ \\
T4 & $0.2 *$ & $0.2 *$ \\
T5 & $0.2 *$ & $0.2 *$ \\
T6 & $0.2 *$ & $0.2 *$ \\
T7 & $0.2 *$ & $0.2 *$ \\
T8 & $0.2 *$ & $0.2 *$ \\
T9 & $0.2 *$ & $0.2 *$ \\
T10 & $0.2 *$ & $0.2 *$ \\
T11 & $0.2 *$ & $0.2 *$ \\
T12 & $0.2 *$ & $0.2 *$ \\
L1 & $0.2 *$ & $0.25 *$ \\
L2 & 0.75 & 0.9 \\
L3 & 1.1 & 1.55 \\
L4 & 1.5 & 1.65 \\
L5 & 1.6 & 1.25 \\
Ref. & 1.2 & 1.8 \\
\hline$*$ Extrapolated data & 4 \\
\hline
\end{tabular}

Table 2: Maximum of endplate concavity $\left(c_{\max }\right)$ per vertebral level

\section{References}

[1] J. Mizrahi, M.J. Silva, T.M. Keaveny, W.T. Edwards, W.C. Hayes, 'Finite-element stress analysis of the normal and osteoporotic lumbar vertebral body'. Spine Vol. 18, pp. 2088-2096, 1993.

[2] N.A. Langrana, S.P. Kale, W.T. Edwards, C.K. Lee, K.J. Kopacz, 'Measurement and analyses of the effects of adjacent end plate curvatures on vertebral stresses'. The Spine Journal Vol. 6, pp. 267-278., 2006.

[3] F.H. Martini (ed) (2004) Fundamentals of anatomy and physiology. 6th edn. Pearson Education, San Frasisco, USA

[4] M.F. Eijkelkamp On the development of an artificial intervertebral disc. PhD thesis, Rijksuniversiteit Groningen/ 2002. 
Summary

Samenvatting

Dankwoord

About the author

Publications 


\section{Summary}

Scoliosis is a three-dimensional deformity of the spine and trunk, comprising of a lateral deviation and an axial rotation. Severe scoliosis is mostly seen in girls and is often detected during adolescence. Without treatment, scoliosis progression can lead to a life-threatening situation because the heart and lungs become oppressed. For these reasons, surgical treatment is used in severe cases of scoliosis to correct the deformity of the spine and trunk.

Within the STW-project "A non-fusion scoliosis correction device" we aim for the development of a new implantable scoliosis correction device. This project consists of three PhD projects:

1) the design and prototyping of a new scoliosis correction implant (Martijn Wessels),

2) in vitro tests on human and porcine spines to determine biomechanical spine properties and animal experiments to test the prototype (Iris Busscher)

3) development of a numerical model of the mechanical behaviour of the spine and trunk to optimize the design of the implant (Gerdine Meijer; this thesis).

Main goal of this thesis is to present the numerical model of an average, adolescent spine and trunk with which we quantify various biomechanical aspects that are important in scoliosis correction. This model will help to optimize the development of the new scoliosis correction implant, and in the future, patient-specific models can be used to determine the optimal properties and surgical techniques for an individual patient.

In addition, the model will also increase our understanding of the biomechanics of the spine and trunk.

A detailed description of the final model and all partial models and their validation processes is presented in chapter 2. The biomechanical behaviour of the developed model of the spine, ribcage and trunk showed behaviour similar to that reported in literature, although validation of the adolescent models and scoliotic models is difficult due to lack of experimental data. No studies into material properties, biomechanical behaviour of the isolated spine or spine including ribcage have been published for the adolescent or scoliotic spine. And only a few in vivo measurements in adolescents are published, often without an accurate description of the loading conditions.

Furthermore, since the true cause of adolescent idiopathic scoliosis is unknown, it is difficult to model the mechanical effects of scoliosis (other than the changing geometry) compared to the healthy spine.

However, results from the current models are consistent with current knowledge concerning the biomechanics of the spine and trunk, the differences in the biomechanics of the spine between adults and adolescents, and the changing kinematics of the scoliotic spine compared to the healthy spine. Therefore the current models are considered to be representative for the passive biomechanical behaviour of the adolescent healthy and scoliotic spine/trunk and can be used to predict the scoliosis correction by the new implant and answer biomechanical questions related to scoliosis correction. 
Since "adolescent idiopathic scoliosis" is the aim of our research, the model has to be representative for an adolescent spine and trunk. However, studies reported on in literature exclusively concern the biomechanics of adult spines. In chapter $\mathbf{3}$, we therefore analysed what the effects of (geometrical) growth are on the biomechanics of a motion segment. We found that not height growth, but especially the increase of the depth and width of the spine has a large influence on the biomechanical behaviour of the spine. Height scaling alone is therefore insufficient; when modelling the biomechanical behaviour of the adolescent spine, three-dimensional scaling of the spine is essential. During adolescent growth, the spinal stiffness increases by about $40 \%$ for all rotational movements, due to changing geometry.

To be able to provide good patient-specific models in the future, it is important to know which patient-specific geometrical aspects have a large influence on the biomechanics of the spine. Therefore, the natural variance in the various dimensions of the spine is determined, and the stiffness's for the minimal and maximal dimensions are compared to the stiffness of a motion segment with the average dimensions (chapter 4). From the results in this chapter it was clear that especially the disc height needs an accurate determination in patient-specific models; the smallest disc height increased the stiffness to $226 \%$ of the average value and the largest disc height decreased the stiffness to $75 \%$ of the average value. The interindividual variance in the cross-sectional areas of the ligaments (except for the capsular ligaments) had negligible influence on the spinal stiffness, and an average representation of these parameters is therefore acceptable, even in patient-specific models.

Since scoliosis affects large parts of the thoracolumbar spine and the ribcage as well, the final model includes the biomechanics of multiple motion segments, the ribs and their interaction. The thoracic vertebrae are connected to the ribs with ligaments, but these ligaments are anatomically intertwined with the spinal ligaments and the intervertebral disc. As a result, the removal of the ribs in in vitro set-ups is timeconsuming and will reduce the quality of the specimen and the validity of the results of these tests. For this reasons, most in vitro tests leave the posterior part of the ribs (about $3 \mathrm{~cm}$ ) attached. Also, the intercostal muscles are not removed. In those tests, it is implicitly assumed that this is still a good representation of the biomechanics of the isolated spine. This hypothesis was tested in chapter 5 using a model containing three thoracic motion segments and the posterior $3 \mathrm{~cm}$ part of the connected ribs. For three models (T1-T4, T5-T8, T9-T12), we compared the biomechanical behaviour with and without these $3 \mathrm{~cm}$ ribs. We found the mechanical behaviour of three motion segments with and without the ribs to be similar, except for lateral bending. In lateral bending, the posterior part of the ribs and the related structures did increase the stiffness with 15-40\% (largest effects for mid-thoracic region). For the biomechanical behaviour of the total thoracolumbar spine, we found that the influence of the posterior rib parts was large for all movement directions, with an increase of the stiffness of $17 \%-130 \%$; again the effects of the rib parts were largest in lateral bending. 
In a scoliotic patient, the deformity is not only limited to the spine, but also the ribcage and total trunk are deformed. The interaction between the spine and the other structures of the trunk thus influence the progression, and likely also the correction, of scoliosis. Any model used to predict scoliosis progression or correction, should therefore not only consider the biomechanical behaviour of the spine, but also incorporate the biomechanics of the ribcage and the other structures of the trunk. Since these effects have not been analysed before, in chapter $\mathbf{6}$, we quantified the effects of the spine, ribcage and intra-abdominal pressure on the stiffness of the adolescent trunk.

The intra-abdominal pressure was modelled as an incompressible volume (with an overpressure) in the shape of the intra-abdominal cavity. In this way, the direct effect of the intra-abdominal pressure on the vertebrae is represented, as well as the upward force on the diaphragm and ribcage, and the passive stiffness of both the abdominal and dorsal muscles. We found that the intra-abdominal pressure accounted for $50 \%-69 \%$ of the total stiffness of the trunk (depending upon the loading direction). The ribcage accounted for $19 \%-25 \%$ and the spine for $10 \%-25 \%$ of the total stiffness of the trunk.

In chapter 7 the performance of the designed implant was tested. The short term and long term effects of the implant on both the lateral and axial deformity are analysed. The simulations show that the short term correction of the new scoliosis correction implant is small, while the long-term correction will be considerable. Only a short implantation period was simulated, however, the implant will correct longer in reality. Since the exact time scale of the relaxation of the soft tissues and the reduction of the moment applied by the implant are unknown, it is currently not possible to simulate this qualitatively.

Based on these simulations, a long-term correction of a mild scoliosis with relative low moments (1.5 Nm torsion) seems feasible. Our result also showed that a torsion implant can be used to correct the lateral deformity in scoliosis. The ratio between the correction of the axial rotation and the lateral deformity of course depends on the patient-specific situation; both the patient-specific ratio between lateral bending and axial rotation stiffness of the trunk and the scoliotic shape will influence the correction outcome.

In this chapter we also compared scoliosis induction with scoliosis correction. Since scoliosis does not occur in animals, most animal experiments induce rather than correct scoliosis. From a mechanical point of view, the comparison between scoliosis correction and induction is however not straight-forward. Therefore, we compared the mechanical behaviour of a healthy spine in which scoliosis is induced to a scoliotic spine in which scoliosis is corrected. Comparison of scoliosis correction and induction in our models showed that the mechanical effects of a torsion loading on a healthy spine differ from the effects on a scoliotic spine. Although both showed effects in axial rotation and lateral deformity, and no effects on the sagittal shape, the effects in the lateral plane were much higher in the scoliosis correction simulations than in the scoliosis induction simulations. This suggests a different relation between the applied torsion loading and the coupled lateral movement for scoliotic and healthy spines. The use of scoliosis induction in animals to predict the outcome of scoliosis correction in humans might therefore not be a valid method, although alternatives are hard to find. 
In chapter 8 the methods and results from this thesis are analysed and their implications for future research are discussed.

In this thesis, a numerical model of an average adolescent trunk was developed and validated. Various biomechanical aspects that are important in scoliosis correction were quantified: the influence of adolescent growth and inter-personal variations in geometry on the stiffness of the spine were analysed, but also the relative contributions of the thoracolumbar spine, ribcage and intra-abdominal pressure to the biomechanics of the trunk were analysed. The outcome of the newly developed scoliosis correction implant was simulated; both the axial rotation and lateral deformity were corrected with an implant that applied a torsion moment. Simulations of multiple iteration steps proved that the correction is increasing over time, due to adaptation and visco-elastic behaviour of the soft tissues. However, no quantitative predictions could be made, since quantitative data on visco-elastic behaviour and stress-adaptation of the soft tissues and relaxation effects of the implant in combination with these soft tissues are lacking.

In the future, the long-term prediction of scoliosis correction by the model might be improved by addition of visco-elastic behaviour and implementation of growth aspects. For a good clinical tool, however, patient-specific modelling, in which especially the disc height needs an accurate determination, seems the most logical first improvement. 


\section{Samenvatting}

Scoliose is een driedimensionale vervorming van de wervelkolom en romp, bestaande uit een laterale verplaatsing en axiale rotatie. Ernstige vormen van scoliose komen vooral voor bij meisjes en worden vaak ontdekt tijdens de pubertijd. Zonder behandeling kan een verergering van scoliose leiden tot een levensbedreigende situatie, omdat hart en longen in de verdrukking komen. In ernstige gevallen van scoliose wordt dan ook een operatie uitgevoerd om de vervorming van de wervelkolom en romp te corrigeren.

Binnen het STW-project "non-fusie scoliosecorrectie implantaat" hebben we als doel een nieuw scoliosecorrectie-implantaat te ontwikkelen. Dit project bestaat uit 3 promotieprojecten:

1) het ontwerpen en het maken van een prototype van het nieuwe scoliose correctie implantaat (Martijn Wessels)

2) in vitro testen met humane en varkens- wervelkolommen om de biomechanische eigenschappen van de wervelkolom te bepalen en dierexperimenten waarin het prototype getest wordt (Iris Busscher)

3) ontwikkelen van een numeriek model van het biomechanische gedrag van de wervelkolom en romp om het ontwerp van het implantaat te optimaliseren (Gerdine Meijer; dit proefschrift).

Hoofddoel van dit proefschrift is het presenteren van het numerieke model van een gemiddelde, tienjarige wervelkolom en romp waarmee verschillende biomechanische aspecten die van belang zijn in scoliosecorrectie, kunnen worden gekwantificeerd. Met behulp van dit model kan het ontwerp van het implantaat worden geoptimaliseerd en, in de toekomst, kunnen patiëntspecifiek modellen gemaakt worden om de optimale instellingen en operatietechniek te bepalen voor een individuele patiënt.

Daarnaast draagt het model bij aan onze algemene kennis over de biomechanica van de wervelkolom en romp.

Een gedetailleerde beschrijving van het totaalmodel en de verschillende deelmodellen alsook van hun validatie processen wordt gegeven in hoofdstuk 2 . Het biomechanische gedrag van de ontwikkelde modellen van de wervelkolom, ribbenkast en romp zijn vergeleken met literatuur en laten vergelijkbaar gedrag zien. Validatie van tienjarige modellen en scoliotische modellen is echter lastig, omdat er weinig experimentele data is. Studies die materiaaleigenschappen, of de biomechanische eigenschappen van de kale wervelkolom, of de wervelkolom met de ribben bepalen, in tiener en/of scoliotische wervelkolommen, zijn niet gepubliceerd. En alleen schaarse in vivo metingen in tieners zijn gepubliceerd, maar vaak zonder duidelijke beschrijving van de belastings-randvoorwaarden.

Bovendien is het moeilijk om de mechanische effecten van scoliose (anders dan de veranderde geometrie) in vergelijking tot de gezonde wervelkolom te modelleren, omdat de oorzaak van adolescente idiopatische scoliose onbekend is.

Resultaten van het huidige modellen zijn echter consistent met de kennis over de biomechanica van de wervelkolom en romp, de verschillen in de biomechanica tussen volwassenen en pubers, en de veranderde kinematica van de scoliotische ten opzichte van de gezonde wervelkolom. De huidige modellen worden daarom representatief geacht voor het passieve, biomechanische gedrag van de tienjarige 
gezonde en van de tienjarige scoliotische wervelkolom/romp en kunnen worden gebruikt om het effect van het nieuwe implantaat te voorspellen en biomechanische vragen te beantwoorden die gerelateerd zijn aan de correctie van scoliose.

Omdat ons onderzoek zich richt op "adolescente idiopatische scoliose", moet het model representatief zijn voor de wervelkolom en romp van een tiener. De literatuur bevat echter alleen studies naar de biomechanica van de volwassen wervelkolom. In hoofdstuk 3, hebben we daarom gekeken welke effecten (geometrische) groei heeft op de biomechanica van een bewegingssegment. Onze resultaten laten zien dat niet de lengtegroei, maar vooral de toename in breedte en diepte van de wervelkolom tijdens de groei een grote invloed heeft op het biomechanische gedrag van de wervelkolom. Het is daarom onvoldoende om alleen de hoogte te schalen; wanneer het biomechanische gedrag van een adolescente wervelkolom wordt gemodelleerd is een driedimensionale schaling van de wervelkolom essentieel. Tijdens de groeispurt neemt de stijfheid ongeveer $40 \%$ toe voor alle rotaties door de veranderingen in de geometrie.

Om, in de toekomst, goede patiëntspecifieke modellen te kunnen maken is het belangrijk om te weten welke inter-individuele variatie in de geometrische aspecten een grote invloed hebben op het biomechanische gedrag van de wervelkolom. Daarom wordt allereerst voor de verschillende geometrische afmetingen de natuurlijke variatie bepaald. Vervolgens wordt de stijfheid van een bewegingssegment met de minimale en maximale afmetingen vergelijken met de stijfheid van een bewegingssegment met de gemiddelde afmetingen (hoofdstuk 4). Uit de resultaten van dit hoofdstuk blijkt dat vooral de hoogte van de tussenwervelschijf erg nauwkeurig moet worden bepaald voor patiëntspecifieke modellen; de kleinste hoogte van de tussenwervelschijf verhoogt de stijfheid tot wel $226 \%$ van de gemiddelde waarde terwijl de grootste hoogte van de tussenwervelschijf de stijfheid verlaagt tot $75 \%$ van de gemiddelde waarde. De interindividuele variatie in de dikte van de ligamenten (het facet kapsel uitgezonderd) hebben een verwaarloosbare invloed op de stijfheid en een gemiddelde afmeting is daarom afdoende, zelfs in patiëntspecifieke modellen.

Omdat scoliose een groot gedeelte van de thoracolumbale wervelkolom en de ribbenkast beïnvloedt, zal het uiteindelijke model de biomechanica van meerdere bewegingssegmenten, de ribben en de interactie hiertussen, moeten beschrijven. De borstwervels zijn via ligamenten verbonden aan de ribben, maar deze ligamenten zijn sterk vergroeid met de ligamenten van de wervelkolom evenals met de tussenwervelschijf. Dit heeft tot gevolg dat het verwijderen van de ribben, in in vitro testopstellingen, erg veel tijd kost, hetgeen ten koste gaat van de kwaliteit van het te testen stuk wervelkolom en de bruikbaarheid van de resultaten. Daarom wordt in de meeste in vitro testen het achterste gedeelte van de ribben (ongeveer $3 \mathrm{~cm}$ ) niet verwijderd. Ook de tussenribspieren worden niet verwijderd. De impliciete aanname is dat dit toch resultaten geeft die representatief zijn voor de kale wervelkolom. Deze hypothese is getest in hoofdstuk 5 met behulp van modellen bestaande uit drie thorocale bewegingssegmenten en de onverwijderde achterste $3 \mathrm{~cm}$ van de ribben. Voor drie modellen (T1-T4, T5-T8 en T9-T12) hebben we het biomechanische gedrag van de modellen met en zonder ribben vergeleken. Onze resultaten laten zien dat het biomechanische gedrag van deze stukken wervelkolom (drie 
bewegingssegmenten) met en zonder de $3 \mathrm{~cm}$ ribben inderdaad vergelijkbaar is, behalve voor laterale buiging. In laterale buiging verhoogt het achterste gedeelte van de ribben en de aanverwante structuren de stijfheid met $15 \%-40 \%$ (grootste effect wordt gevonden voor de mid-thorocale regio). Voor het biomechanische gedrag van de totale thoracolumbale wervelkolom, blijkt de invloed van de $3 \mathrm{~cm}$ ribben voor alle bewegingsrichtingen groot te zijn, met $17 \%-130 \%$ verhoging van de stijfheid, waarbij ook hier het grootste effect wordt gezien in laterale buiging.

In een patiënt met scoliose beperkt de vervorming zich niet alleen tot de wervelkolom, maar ook de ribbenkast en de romp zijn vervormd. De interactie tussen de wervelkolom en de ribben beïnvloedt dus de progressie, en waarschijnlijk ook de correctie, van scoliose. Een model dat wordt gebruikt om scoliosecorrectie te voorspellen zou daarom niet alleen het biomechanische gedrag van de wervelkolom moeten beschouwen, maar ook het biomechanische gedrag van de ribben en andere structuren van de romp. Omdat deze effecten nog niet eerder onderzocht zijn, kwantificeren we in hoofdstuk 6 de invloed van de wervelkolom, de ribbenkast en de interne buik druk op de stijfheid van de tienjarige romp.

De buikdruk wordt hierbij gemodelleerd als een onsamendrukbaar volume (overdruk) in de vorm van de intra-abdominale ruimte. Hierdoor wordt zowel het directe effect van de buikdruk op de wervels gemodelleerd, als de opwaartse kracht op het diafragma en de ribbenkast, als de passieve stijfheid van de buik- en rugspieren. We hebben gevonden dat de buikdruk een relatieve bijdrage levert van 50\%-69\% aan de stijfheid van de romp (afhankelijk van de belastingsrichting). De ribbenkast draagt $19 \%-25 \%$ bij en de wervelkolom draagt $10 \%-25 \%$ bij.

In hoofdstuk 7 wordt de werking van het ontworpen implantaat getest. De korte- en lange-termijn correctie voor de laterale en axiale vervorming worden geanalyseerd. De simulaties laten zien, dat hoewel de korte termijn correctie van het implantaat beperkt is, de lange termijn correctie aanzienlijk zal zijn. In de analyses is slechts een korte tijdsperiode gesimuleerd, maar het implantaat zal in werkelijkheid langer blijven corrigeren. Echter, omdat de exacte tijdsschaal van de relaxatie en het effect op het geleverde moment door het implantaat onbekend zijn, is dit momenteel niet goed te simuleren.

Gebaseerd op de huidige simulaties lijkt een lange-termijn correctie van een milde scoliose met kleine momenten ( $1.5 \mathrm{Nm}$ axiale torsie) zeer zeker haalbaar. Onze resultaten laten ook zien dat een torsie-implantaat gebruikt kan worden om de scoliotische laterale vervorming te corrigeren. De verhouding tussen de axiale en laterale correctie is uiteraard patiëntspecifiek; zowel de patiëntspecifieke verhouding tussen de laterale buigstijfheid en torsie stijfheid van de romp als de vorm van de scoliose zal de uiteindelijke correctie bepalen.

In dit hoofdstuk hebben we ook scoliosecorrectie en scolioseinductie vergeleken. Omdat scoliose niet voorkomt in dieren, wordt er in dierexperimenten vaak scoliose geïnduceerd in plaats van gecorrigeerd. Mechanisch gezien, is het vergelijken van scoliosecorrectie en -inductie echter niet eenduidig. Daarom hebben we het mechanisch gedrag van een gezonde romp waarin scoliose gecreëerd wordt vergeleken met een scoliotische romp waarin scoliose gecorrigeerd wordt. Vergelijking van scoliosecorrectie en scolioseinductie met onze modellen liet zien dat de mechanische effecten van een torsiebelasting op een gezonde romp en een scoliotische romp niet hetzelfde zijn. Hoewel er voor beiden een effect te zien was 
voor axiale rotatie en in het laterale vlak, en geen effect op de sagittale vorm, was het effect in het laterale vlak voor de scoliosecorrectie-simulaties veel groter dan voor scolioseinductie-simulaties. Dit impliceert een ander gedrag voor de gekoppelde laterale beweging bij een torsiebelasting voor de gezonde en de scoliotische wervelkolom. Het gebruiken van scolioseinductie in dieren om de scoliosecorrectie in mensen te voorspellen lijkt daarom geen valide methode, maar alternatieven zijn moeilijk te vinden.

In hoofdstuk 8 worden de methodes en resultaten uit dit proefschrift en de gevolgen voor toekomstig onderzoek beschouwd.

Een numeriek model van een gemiddelde tienjarige wervelkolom is ontwikkeld en gevalideerd in dit proefschrift. Verscheidene biomechanische aspecten die belangrijk zijn in scoliosecorrectie zijn gekwantificeerd: de invloed van de groeispurt en interpersoonlijke verschillen in geometrie op de stijfheid van de wervelkolom zijn geanalyseerd, maar ook de bijdrage van thoracolumbale wervelkolom, de ribbenkast en de buikdruk aan de biomechanische eigenschappen van de romp zijn bepaald. Het effect van het nieuwe scoliosecorrectie-implantaat is gesimuleerd; zowel de axiale rotatie als de laterale vervorming wordt gecorrigeerd met behulp van een torsie-implantaat. Simulaties met meerdere iteratie stappen bewijzen dat de correctie met de tijd toeneemt, door de adaptatie en visco-elastisch gedrag van de zachte weefsels. Kwantitatieve voorspellingen zijn echter nog niet mogelijk, omdat kwantitatieve data over visco-elastisch gedrag en stress-adaptatie van de zachte weefsels en de relaxatie-effecten van het implantaat in combinatie met deze zachte weefsels niet bekend zijn.

De lange-termijn scoliosecorrectie die wordt voorspeld door het model kan in de toekomst verbeterd worden door het toevoegen van visco-elastisch gedrag en groeiaspecten. Voor een goed gebruik in de kliniek lijkt echter het maken van patiëntspecifieke modellen, waarin vooral de hoogte van de tussenwervelschijf nauwkeurig bepaald moet worden, een logische eerste stap. 

Mijn dankwoord wil ik graag beginnen met een Afrikaans spreekwoord:

'Als je snel vooruit wil, ga dan alleen. Als je ver wil komen, ga dan samen.'

En er zijn heel veel mensen die er voor gezorgd hebben dat dit onderzoek niet alleen ver is gekomen, maar ook sneller de goede kant op ging.

Allereerst wil ik mijn promotoren, Prof.dr.ir. G.J.Verkerke en Prof.dr. A.G.Veldhuizen bedanken.

Beste Bart, de wekelijkse discussies waren soms misschien wat heftig, maar altijd opbouwend. Ik heb vooral veel van je geleerd over het samenwerken binnen multidisciplinaire projecten, wat ik in de toekomst nog vaak hoop te doen. Ook heb je me geleerd dat het altijd alles verder willen uitzoeken niet per se leidt tot beter onderzoek, maar soms gewoon duidt op gebrek aan vertrouwen. En dit vertrouwen had jij altijd wel! Dank voor je prettige begeleiding, het geduld tijdens het hele proces en je sarcastische humor.

Beste Albert, hartelijk dank voor de belangrijke klinische inbreng in dit proefschrift. De discussies hebben er aan bijgedragen dat ik wat meer begrijp van de ruggengraat wervelkolom, scoliose en groei, en ze hebben er voor gezorgd dat het uiteindelijke model relevant en representatief is voor de doelgroep. Bedankt voor de snelle en positieve reacties, en op naar een patient-specifiek model, toepasbaar in de kliniek!

Mijn assistent promotor en dagelijks begeleider, dr. ir. J. Jomminga.

Beste Jasper, misschien heb ik dat dagelijks soms iets te letterlijk genomen, maar je deur stond altijd open. Erg fijn, niet alleen dat ik zo vaak mocht binnenlopen, maar ook je manier van begeleiden: heel open, waardoor het bijna niet als begeleiden voelt, maar juist erg effetcief is voor koppige vastbijters en hopeloze in-detailverzanders als ik. En dank voor alle mensenkennis en geduld die je hebt ingezet tijdens mijn motivatie-dipjes, praktische-bezwaren-dagen en algehele-dwarsheidstemmingen. Daarnaast dank voor de vele gesprekken over van alles en de toekomst, gezellige tripjes naar een project-vergadering ergens in het land, of overleg in Parijs, of congressen in Europa, en last but not least, dank voor het leggen van de eerste contacten voor een postdoc-plek.

Leuk dat we weer samenwerken in het volgende project!

Mijn PhD-collega's in dit project, ir. M. Wessels en dr. I. Busscher.

Beste Martijn, het doel van onze samenwerking was dat mijn model zou leiden tot een optimalisatie van jouw ontwerp, maar al snel was duidelijk dat dit plantechnisch niet te realiseren was. Dat maakte de discussies in onze kamer over het onderzoek, en allerlei niet-werk gerelateerde onderwerpen, er niet minder leuk en effectief om. En waar zou dit proefschrift geweest zijn zonder jouw geduld en hulp als er weer eens een hopeloos probleem was met mijn computer! Ook bedankt voor je lessen in het geven van zinnig voetbal-commentaar, terwijl je er eigenlijk geen verstand van hebt (Werkt erg goed!). En natuurlijk dank voor alle gezellige autoritjes naar Groningen, congresavonden en biertjes. Op naar jouw feestje, heb nu al zin!

Beste Iris, als eerste dr.! Ik heb erg veel respect voor je doorzettingsvermogen en ambitie, zeker als het gaat om het halen van onmogelijke deadlines en het gewoon "aanpakken", ook als het nog niet te overzien is waar het naar toe gaat. Zonder jouw aanpak, had het zeker langer geduurd voordat er data was om het model mee te kunnen valideren. En dank voor de vele gezelligheid tussen het samenwerken door: winkelen, lunchen, dineren en logeren in Groningen/Amsterdam. 
Great thanks to Paul Groenenboom and Muriel Beaugonin, from ESI, for their support, whenever I had questions about the software or finite element modelling in general. Your answers were always elaborate, fast and very helpful. Without your help, I would not have gotten this far.

Edsko Hekman, bedankt voor je oplossingsgerichte aanpak, kritische opmerkingen en het actief meeschrijven aan de verschillende hoofdstukken. Altijd efficiënt, maar daarom niet minder doordacht of gezellig!

Albert van der Veen, Jaap van Dieën, Idsard Kingma en Theo Smit: dank voor de uitgebreide (email)discussies, over met name experimenten en hun representatie van de werkelijkheid. Er kwam niet altijd een duidelijk antwoord uit, maar wel altijd heel veel interessante nieuwe vragen en meer begrip van de complexiteit van het probleem. Dank dat ik mocht meekijken in de keuken van de experimenten! En Albert, tot op het volgende congres; wordt het vast weer erg leuk!

Marc Sanders en Gert Nijenbanning, hartelijk dank voor het delen van jullie ervaring en kennis over/in het ontwerpen van een scoliose correctie systeem en het modelleren van de wervelkolom.

Bart Koopman, dank voor het advies over van alles en nog wat: van "Is een promotie wel wat voor mij?" tot "Hoe modeleer ik passieve mechanische spier eigenschappen?". Het is altijd leuk en zinvol om met je te discussiëren.

Timo Meinders bedankt voor een helder opfris-college eindige elementen methode.

Nico Verdonschot bedankt voor de discussies over het modelleren van de wervelkolom, de (on)mogelijkheden hiervan (al in het begin van het project, toen vond ik het allemaal nogal onheilspellend klinken) en het altijd meedenken.

Prof. dr. D.Uyttendaele, Prof. dr. ir. D.J.Schipper, Prof. dr. T.G. van Kooten, Prof. dr. G. Rakhorst, Bas van Riggelen, Rob Slootman, Nico Boots en Henry van der Valk, dank voor de kritische vragen tijdens de STW-overleggen en jullie tijd en interesse!

Lianne en Wies bedankt voor het draaiend houden van de vakgroep met een glimlach. En vooral bedankt voor het luisterend oor, de korte peptalks en de goede tips over alles in het leven, jullie zijn echt een beetje de vakgroep-mama's!

Nikolai bedankt voor je hulp bij alle grote (en achteraf toch vaak kleine) computerproblemen!

Ik wil graag alle studenten bedanken die hun bachelor, master of een andere opdracht hebben gedaan in het kader van dit onderzoek, en wiens resultaten direct of indirect hebben geleid tot dit proefschrift. In chronologische volgorde: Jean Paul Klein, Tim Bost, Jos Boeije, Jordy Mongula, Annemarie Schrijver, Mechteld Lehr, Jolanda van Eijden, Bart Pater, Jan de Jong, Heimen Vos, Christine Huizer, Jean Paul Klein, Claudia Haarman, Hielke Kiewiet, Charlotte Avis, Josien Beijer en Mechteld Lehr. Doordat jullie allemaal je eigen werkwijze hadden en nieuwe kennis hebben ingebracht over het modelleren, de wervelkolom en/of scoliose is mijn blik op deze onderwerpen én het aanpakken van problemen veel breder geworden. Dank dat ik van jullie mocht leren en dank dat jullie het onderzoek nog leuker maken!

Alle (ex-)medewerkers en studenten van biomedische werktuigbouwkunde bedankt voor de gezelligheid. Het is een groep waar iedereen zich zelf mag zijn én is, fantastisch! Bedankt voor de koffie-pauzes, de lunchwandelingen, samen sporten, terras of daghap doen, vakgroepuitjes (wanneer is de volgende ?!?), feestjes, BBQ's gangdiscussies en natuurlijk het samenwerken. Het is inmiddels zo'n grote groep, dat het onbegonnen is alle namen te noemen, daarom: allemaal bedankt voor de belangrijkste secundaire arbeidsvoorwaarde: plezier op je werk! 

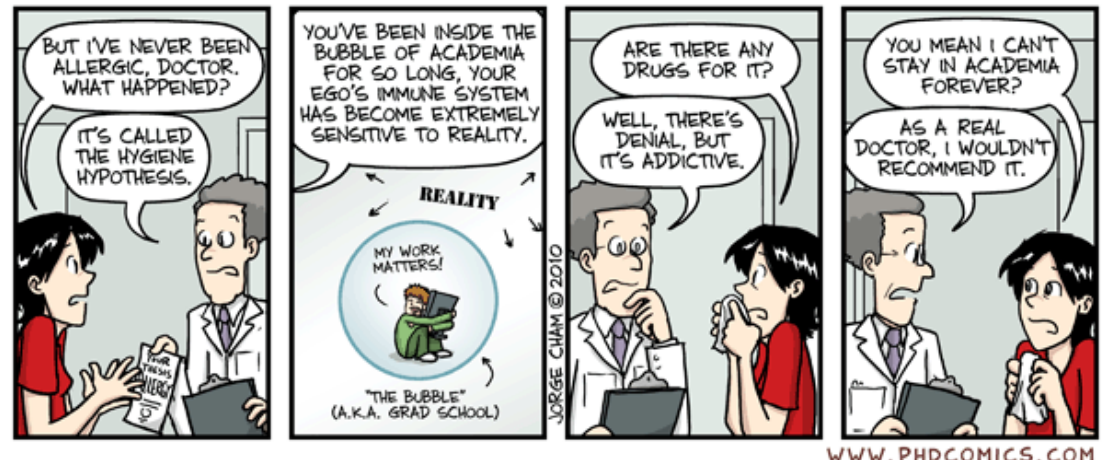

Naast alle mensen in de bubbel, zijn er heel veel mensen van buiten de bubbel, die ik dankbaar ben voor het luisteren naar mijn nerd-verhalen, maar nog veel meer voor het zorgen dat ik niet allergisch word voor de realiteit. De relativerende opmerkingen, vragen en vooral veel gezelligheid hebben er voor gezorgd dat het allemaal in balans bleef. Ik wil hiervoor in het bijzonder bedanken: Anne Corine, Esther, Evelien, Jeroen, Jorien, Maarten, Peter, Sander en Tjitske.

Sanne, Wouter en Tjerk bedankt voor het leegmaken van mijn hoofd, en het met heel veel energie en plezier uitdragen van jullie sport. Elke les is weer een feestje!

Wilco bedankt voor het inspireren tot promoveren, met een interessante verdediging, zoiets wonderlijks als paranimfen (de échte reden dat ik ging promoveren), een inspirerend feestje, en het verhaal dat promoveren net studeren is... wat wil je nog meer! Daarnaast dank voor de vele tips\&trics voor mijn boekje, je voorbeeld als doorzetter en het bewijs dat medische statistiek highly overrated is!

Mijn (schoon)familie: Papa en Mama, Minke en Hille, Ben en Monique, Thalina en Henny, Welmoed en Rutger, Redmar en Bienvennue. Last but not least: Tijs. (Niets is zo relativerend en leuk als met lego spelen!)

Bedankt dat jullie er altijd zijn. Als er leuke verhalen zijn, dan zijn jullie trots, als er feestjes zijn, dan zijn jullie blij, als ik verdietig ben, dan troosten jullie mij. Wat er ook gebeurt, jullie zijn er en hebben vertrouwen in mij!

Anne Corine, Evelien en Tjitske, bedankt dat jullie altijd enthousiast zijn én nadenken over praktische oplossingen, vooral als ik het soms even niet zie (zitten). Jullie zorgen dat vandaag een onbezorgd feestje wordt!

En dan is de laatste plaats natuurlijk voor Quirijn. Altijd daar, onvoorwaardelijk stabiel. Zonder jouw woorden, "Misschien is promoveren wel wat voor jou?" , was ik hier misschien nooit aan begonnen. En zonder je lichtelijke duwtjes ("En wat ga je er nu aan doen?", "Kan het niet anders dan?") was ik misschien minder ver gekomen. Maar zonder je relativerende opmerkingen en humor was het zeker veel minder leuk geweest. De "data-heks op de bank", vrij naar het Sprookjeshuwelijk van Jochem Myjer, en de "FEM fatale", maken dat ik altijd moet lachen, hoe slecht het onderzoek en/of mijn humeur er ook aan toe zijn.

Daarom: "De wereld is mooier met jou". 



\section{About the author}

Gerdine Meijer was born on December $6^{\text {th }} 1978$ in Oldenzaal, the Netherlands.

After she finished high school (VWO) in 1997, she first studied dentistry (Radboud University Nijmegen), but after one year she switched to mechanical engineering (University of Twente). Her masters had a specialization in biomechanical

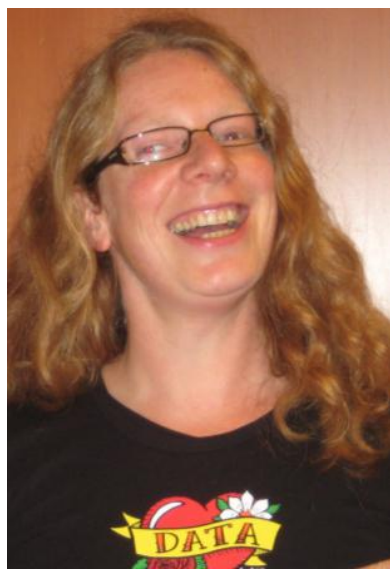
engineering. During this master, she did a research internship at the Biomechatronics Lab of the University of Limerick, Ireland. During this research, the usability of accelerometers in fall detection of elderly people was analysed, using multi-segment models. This project was supervised by Prof. dr. G. Lyons and Prof. dr. ir. H.F.J.M.Koopman.

She did the research for her master thesis at the TWente Institue for Neuromodulation (TWIN), which was titled "Design of a FNS -method and -system to improve walking after quadriceps weakness caused by a stroke". During this research, the feasibility of Functional Electrical Stimulation of the femoral nerve in stroke patients was analysed, by combining volume conductor models of the nerve and bond graph models of the leg. This project was supervised by Dr. R. Buschman and Prof. dr. ir. H.F.J.M.Koopman.

Her interests in using technology and modelling to improve health care lead her to a PhD position at the lab of Biomechanical Engineering at the University of Twente (2007-2011). In the project "A non-fusion scoliosis correction device", a finite element model of the spine and trunk was made. The model was used to analyse a new scoliosis correction implant that was developed during the same project. The project was supervised by Prof.dr.ir. G.J.Verkerke, Prof.dr A.G. Veldhuizen and dr. ir. J. Homminga. This thesis is based on the work done during this project.

From January 2011, Gerdine works as a postdoctoral researcher at both the University of Twente and the Technical University of Eindhoven, for the project "Spineguide". In this project a new surgical technique for scoliosis correction is analysed and developed, using numerical modelling of scoliosis correction. 


\section{International Journal Papers}

- $\quad$ G.J.M. Meijer, J. Homminga, E.E.G. Hekman, A.G. Veldhuizen, G.J. Verkerke. 'The effect of three-dimensional geometrical changes during adolescent growth on the biomechanics of a spinal motion segment'. J Biomech Vol. 43, pp. 15901597, 2010.

- I. Busscher, J.H. van Dieën, A.J. van der Veen, I. Kingma, G.J.M. Meijer, G.J. Verkerke, A.G. Veldhuizen. 'The effects of creep and recovery on the in vitro biomechanical characteristics of human multi-level thoracolumbar spinal segments'. Clinical Biomechanics Vol. 26, pp. 438-444, 2011.

- $\quad$ G.J.M. Meijer, J. Homminga, A.G. Veldhuizen, G.J. Verkerke. 'Influence of interpersonal geometrical variation on spinal motion segment stiffness: Implications for patient-specific modeling'. Spine Vol. 36, pp. E929-E935 2011.

- $\quad$ G.J.M. Meijer, J. Homminga, G.J. Verkerke. 'Influence of costovertebral joints on the stiffness of the spine'. Submitted.

- G.J.M. Meijer, J. Homminga, G.J. Verkerke. 'Mechanical role of the spine, ribcage and intra-abdominal pressure in the behaviour of the trunk'. Submitted.

- G.J.M. Meijer, M. Wessels, E.E.G. Hekman, J. Homminga, G.J. Verkerke. 'Is scoliosis induction a good model for scoliosis correction?'. To be submitted.

- $\quad$ M.S. Sietsma, G.J.M. Meijer, N. Verdonschot, J. Homminga, A.G. Veldhuizen. 'Altered sagittal alignment, consequences on spinal loads and trunk muscle forces; a finite-element study'. To be submitted.

\section{Conference participations}

- G.J.M. Meijer, J. Homminga, C. Obbink-Huizer, G.J. Verkerke. 'Mechanical influence of spine, ribcage and interabdominal pressure on the ten-year old trunk'. 3rd Dutch Conference on Bio-Medical Engineering: Egmond aan Zee, The Netherlands, 2011. Oral presentation.

- $\quad$ G.J.M. Meijer, C. Obbink-Huizer, J. Homminga, G.J. Verkerke. 'Influence of the ribcage on the mechanical behaviour of the ten-year old spine'. 17th Congress of European Society of Biomechanics: Edinburgh, United Kingdom, 2010. Poster presentation.

- G.J.M. Meijer, J. Homminga, G.J. Verkerke. 'Influence of interpersonal geometrical variation on spinal stiffness'. 17th Congress of European Society of Biomechanics: Edinburgh, United Kingdom, 2010. Oral presentation.

- $\quad$ G.J.M. Meijer, J. Homminga, E.E.G. Hekman, A.G. Veldhuizen, G.J. Verkerke. 'Influence of growth on spinal stiffness' . 2nd Dutch Conference on Bio-Medical Engineering: Egmond aan Zee, The Netherlands, 2009. Oral presentation.

- $\quad$ G.J.M. Meijer, J. Homminga, E.E.G. Hekman, A.G. Veldhuizen, G.J. Verkerke. 'Effects of growth and interpersonal geometry differences on spinal stiffness'. 16th Congress of European Society of Biomechanics: Lucerne, Switzerland, 2008. Poster presentation.

- J. Homminga, J.R. Boeije, G.J.M. Meijer, E.E.G. Hekman, J.H. van Dieën, A.G. Veldhuizen, G.J. Verkerke. 'Will we need patient specific spine models?' EuroSpine 2007: Brussels, Belgium, 2007. Poster presentation. 